https://helda.helsinki.fi

\title{
The Narratology of Comic Art
}

\section{Mikkonen, Kai}

Routledge

2017-05-08

Mikkonen , K 2017 , The Narratology of Comic Art . Routledge Advances in Comics Studies , Routledge , New York . https://doi.org/10.4324/9781315410135

http://hdl.handle.net/10138/312420

https://doi.org/10.4324/9781315410135

cc_by_nc_nd

publishedVersion

Downloaded from Helda, University of Helsinki institutional repository.

This is an electronic reprint of the original article.

This reprint may differ from the original in pagination and typographic detail.

Please cite the original version. 
ROUTLEDGE ADVANCES IN COMICS STUDIES

The Narratology of Comic Art Kai Mikkonen 


\section{The Narratology of Comic Art}

By placing comics in a lively dialogue with contemporary narrative theory, The Narratology of Comic Art builds a systematic theory of narrative comics, going beyond the typical focus on the Anglophone tradition. This involves not just the exploration of those properties in comics that can be meaningfully investigated with existing narrative theory, but an interpretive study of the potential in narratological concepts and analytical procedures that has hitherto been overlooked as well. This research monograph is, then, not an application of narratology in the medium and art of comics, but a revision of narratological concepts and approaches through the study of narrative comics. Thus, while narratology is brought to bear on comics, equally comics are brought to bear on narratology.

Kai Mikkonen is Associate Professor of Comparative Literature at the University of Helsinki, Finland. 
Routledge Advances in Comics Studies

Edited by Randy Duncan, Henderson State University

Matthew J. Smith, Radford University

1 Reading Art Spiegelman

Philip Smith

2 The Modern Superhero in Film and Television

Popular Genre and American Culture

Jeffrey A. Brown

3 The Narratology of Comic Art

Kai Mikkonen 


\section{The Narratology of Comic Art}

Kai Mikkonen 
First published 2017

by Routledge

711 Third Avenue, New York, NY 10017

and by Routledge

2 Park Square, Milton Park, Abingdon, Oxon OX14 4RN

Routledge is an imprint of the Taylor \& Francis Group, an informa business

(C) 2017 Taylor \& Francis

The right of Kai Mikkonen to be identified as author of this work has been asserted by him in accordance with sections 77 and 78 of the Copyright, Designs and Patents Act 1988.

All rights reserved. No part of this book may be reprinted or reproduced or utilised in any form or by any electronic, mechanical, or other means, now known or hereafter invented, including photocopying and recording, or in any information storage or retrieval system, without permission in writing from the publishers.

Trademark notice: Product or corporate names may be trademarks or registered trademarks, and are used only for identification and explanation without intent to infringe.

\section{Library of Congress Cataloging-in-Publication Data} CIP data has been applied for.

ISBN: 978-1-138-22155-0 (hbk)

ISBN: 978-1-315-41013-5 (ebk)

Typeset in Sabon

by codeMantra 


\section{Contents}

List of Figures vii

Acknowledgements $\quad$ xi

Introduction: Comics, Narrative, and Medium 1

PART I

Time in Comics 31

1 Time in Comics 33

PART II

$\begin{array}{ll}\text { Graphic Showing and Style } & 71\end{array}$

2 Narration as Showing 73

3 Character as a Means of Narrative Continuity 90

4 Graphic Style, Subjectivity, and Narration 109

PART III

$\begin{array}{ll}\text { Narrative Transmission } & 127\end{array}$

5 Narrative Agency (in Jiro Taniguchi's A Distant Neighborhood) 129

6 Focalisation in Comics 150

7 Characterisation in Comics 174 
vi Contents

PART IV

Speech and Thought in Narrative Comics

8 Presenting Minds in Comics

9 Dialogue in Comics: Medium-Specific Features and Basic Narrative Functions

PART V

Narrative Form and Publication Format

10 Picture Story and Narrative Organisation in Early Nineteenth-Century British Caricature and Comic Strips

Afterword

Bibliography

Index 


\section{List of Figures}

I.1 "After that it was my turn" () Matti Hagelberg. 1

1.1 “April 20th, 2005.” Building Stories (2012) @ Chris Ware. 35

1.2 Extrait de l'ouvrage. A Taste of Chlorine (2011). Bastien Vivès (C) Casterman. Avec l'aimable autorisation des auteurs et des Editions Casterman. $\quad 39$

1.3 Matti Hagelberg. Kekkonen (2004) @) Matti Hagelberg. 44

1.4 Fred. Philémon. La mémémoire (1977) @ Philémontome 11, La mémémoire DARGAUD by Fred. All rights reserved www.dargaud.com.

1.5 Guy Delisle. Pyongyang: A Journey in North Korea (C) 2006 L'Association/Guy Delisle; courtesy of Drawn \& Quarterly.

1.6 Extrait de l'ouvrage A Taste of Chlorine (2011). Bastien Vivès (C) Casterman. Avec l'aimable autorisation des auteurs et des Editions Casterman. 62

2.1 John Leech. "Substance and Shadow" (1843). 78

2.2 H.M. Bateman. "The One-Note Man” (1921). 79

2.3 H.M. Bateman. "The One-Note Man” (1921). 80

3.1 Brian K. Vaughan and Fiona Staples. Saga (C) 2014 Brian K. Vaughan \& Fiona Staples.

3.2 Robert Kirkman, Charlie Adlard, and Cliff Rathburn. The Walking Dead 4 (2005) (C) Robert Kirkman. 95

3.3 John Tenniel. "How Mr. Peter Piper Enjoyed a Day’s 'Pig-Sticking”" (Near Burhampoor, Bengal)' (1853). 98

3.4 John Tenniel. "Mr. Spoonbill's Experiences in the Art of Skating" (1855).

4.1 Tommi Musturi. Walking with Samuel (2009)

(C) Tommi Musturi.

4.2 Winshluss. Pinocchio (2008) @ () Winshluss. 117

4.3 Winshluss. Pinocchio (2008) () Winshluss. 118

4.4 Manu Larcenet. Blast 4 (C) Blast-tome 4, Pourvu que les bouddhistes se trompent DARGAUD by Larcenet. All rights reserved www.dargaud.com. 
4.5 Tommi Musturi. Walking with Samuel (2009)

(C) Tommi Musturi.

5.1 Taniguchi, Jiro. A Distant Neighborhood. Vol. 1. (2009) (C) 1998 Jiro TANIGUCHI @ Fanfare/Ponent

Mon, 2016 for the English edition. www.ponentmon.com. 140

5.2 Taniguchi, Jiro. A Distant Neighborhood. Vol. 1. (2009) () 1998 Jiro TANIGUCHI (C) Fanfare/Ponent Mon, 2016 for the English edition. www.ponentmon.com.

5.3 Taniguchi, Jiro. A Distant Neighborhood. Vol. 1. (2009) () 1998 Jiro TANIGUCHI (C) Fanfare/Ponent

Mon, 2016 for the English edition. www.ponentmon.com.

5.4 Taniguchi, Jiro. A Distant Neighborhood. Vol. 1. (2009) (c) 1998 Jiro TANIGUCHI @ Fanfare/Ponent

Mon, 2016 for the English edition. www.ponentmon.com.

145

6.1 Jarmo Mäkilä. Taxi van Goghin korvaan (2008) (C) Jarmo Mäkilä.

6.2 Emmanuel Guibert, Frédéric Lemercier, and Didier Lefèvre. The Photographer (C) 2009 by First Second.

6.3 From The Arrival by Shaun Tan. Scholastic, Inc./ Arthur A. Levine Books. Copyright () 2006 by Shaun Tan. Reproduced with permission.

7.1 Gary Panter. Jimbo's Inferno (C) 2006 by Gary Panter.

7.2 Guido Martina and Angelo Bioletto. Mickey's Inferno (1949) (C) Disney.

7.3 Guido Martina and Angelo Bioletto. Mickey's Inferno (1949) (C) Disney.

7.4 Glyn Dillon. The Nao of Brown (C) 2012 Glyn Dillon. 191

8.1 Rodolphe Töpffer. Monsieur Vieux Bois (1827). 205

8.2 Charlier and Giraud. Blueberry: Le Spectre aux balles d'or (C) Blueberry-tome 12, Le spectre aux Balles d'or DARGAUD by Giraud and Charlier. All rights reserved www.dargaud.com.

8.3 Madame Bovary par Daniel Bardet et Michel Janvier (C) Éditions Adonis, 2008.

9.1 Abel Lanzac and Christophe Blain. Weapons of Mass Diplomacy (C) DARGAUD 2010, by Blain (Christophe), Abel Lanzac.

9.2 Aapo Rapi. Meti (2008) () Aapo Rapi.

9.3 Garth Ennis and Steve Dillon. PREACHER. Book One (1995) () Garth Ennis and Steve Dillon. All characters, the distinctive likenesses thereof, and all related elements are trademarks of Garth Ennis and Steve Dillon.

9.4 Jérôme Mulot and Florent Ruppert. Barrel of Monkeys (C) 2008, Ruppert, Mulot and L'Association, Rebus Books for the English translation. 
10.1 George Cruikshank and J. Pxxxy’s “Back \& Front View of the Ladies Fancy-Man, Paddy Carey O'Killus" (1822). Courtesy of The Lewis Walpole Library, Yale University.

10.2 Charles Jameson Grant. Every Body's Album 15 September 1834. (C) The British Library Board, LOU. LON 1052.

10.3 William Heath. "Life of a Soldier". Northern Looking Glass XII. 12 December 1825. (C) The British Library Board, P.P.6223.dba.

10.4 William Heath. William Heath. "Life of an Actress". Northern Looking Glass X. 14 November 1825.

(C) The British Library Board, P.P.6223.dba.

10.5 Robert Seymour. "Colonial Slavery". The Looking Glass, No. 8, 1 August 1830. (C) The British Library Board, RB.31.c.31.

10.6 Robert Seymour. "St. Michael of London". The Looking Glass, No. 8, 1 August 1830. (C) The British Library Board, RB.31.c.31. 
This page intentionally left blank 


\section{Acknowledgements}

The writing of this book would have been impossible without the University of Helsinki Visiting Fellowship at Clare Hall College, University of Cambridge, 2014-2015. I am deeply grateful to Maria Nikolajeva, Professor of Education and Director of Children's Literature Centre of Homerton College, for helping to make that happen. My special thanks are also due to John Calton, Matti Hagelberg, Essi Varis, Philip Jenkins, and Mark Shackleton, and the anonymous readers of Routledge Advances in Comics Studies, for reading earlier drafts of this work. Thank you, Matti, for your artwork and the enjoyable experience of teaching a course together on comics storytelling at the University of Helsinki in 2013. Over the past decade, my work has profited greatly from the input from colleagues and friends involved in the Nordic Network for Comics Research (NNCORE) and the comics studies research committee of the International Comparative Literature Association (ICLA). I am very grateful for the sense of scholarly community and real friendship that I have enjoyed with the members of these two groups.

Portions of earlier versions of some chapters have first appeared in the journals Partial Answers 6.2 (2008), American Studies/Amerikastudien 56.4 (2011), International Journal of Comic Art 13.2 (2011), and Scandinavian Journal of Comic Art 1 (2012), and in the anthologies The Rise and the Reason of Comics and Graphic Literature (Eds. Joyce Goggin and Dan Hassler-Forest, 2010), From Comic Strips to Graphic Novels (Eds. Daniel Stein and Jan-Noël Thon, 2013, 2nd edition 2015), and Dialogue across Media (Eds. Jarmila Mildorf and Bronwen Thomas, 2017). I would like to express my gratitude to the publishers and the editors for their permission to reproduce this material here. In the same vein, I would like to extend great thanks to all the copyright holders who have generously given the permission to reproduce the images included here. 
This page intentionally left blank 


\section{Introduction}

Comics, Narrative, and Medium

I first learned to read by reading comics with my father when I was around 5 or 6 years old. For all I know, my father never read comics by himself, except perhaps when browsing comic strips in his morning newspaper, but he wanted to teach me how to read. That's why we ended up reading my comic books. He would hyphenate all the words, syllable by syllable, in the speech balloons with his marker pen on some randomly chosen page and then read the words to me one syllable at a time (Figure I.1).
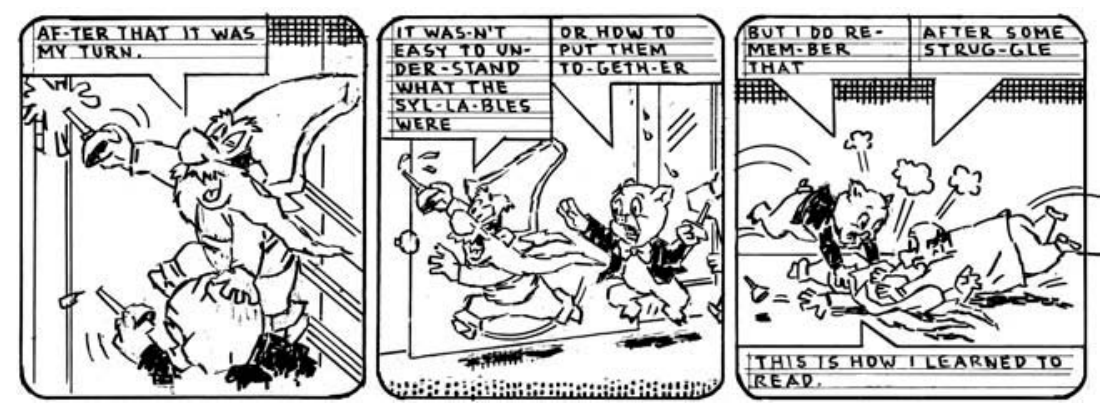

Figure I.1 "After that it was my turn" (c) Matti Hagelberg.

Later I have often wondered what it was in these children's comics, possibly Donald Duck or Bugs Bunny, or in my father's method, that helped me to learn. One reason, definitely, must be the images that I could follow on my own and that I had already encountered. It was exciting to make the connection between the words and the drawings, the characters who were speaking and the situation at hand. The words added new significance to the images and shifted my previous understanding, but I am convinced that the narrative form of these comic books also had something to do with it. Learning the words enhanced the story, making it fuller, perhaps funnier, more thrilling, and more nuanced, while in pleasant symbiosis, the continuing story told in images 


\section{Introduction}

made it easier to figure out what the words meant and how they related to everything that I could see in the images.

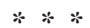

This book has two main objectives. First, by placing comics into lively dialogue with narrative theory, The Narratology of Comic Art builds a systematic theory of narrative comics. This involves not just the identification of those properties in comics that can be meaningfully investigated by means of narrative theory, but a study of the potential in the narratological approach that has hitherto remained overlooked in this field as well. Comics studies is experiencing an exciting period of growth and diversification, and as interest in exploring the connections between comics and narrative theory increases, it is all the more important to carefully think through what is specific to the medium. A comprehensive narratological engagement with comics will allow us to do just that, and contribute to our understanding of comics' narrative devices, conventions, and strategies.

Second, the development of the narratology of comic art entails the identification of the kinds of problems that we encounter in employing narrative theory in comics studies. Many of these problems stem from the historical fact that narratology — the formal, systematic study of narrative representation-has been more extensively developed in literary research than in other fields. This book, then, is not a mere application of existing narratology to the medium, but it also offers a revision of narratological concepts and approaches through the study of narrative comics. Thus, while narrative theory is brought to bear on comics, comics are equally brought to bear on narrative theory.

In this investigation, the relation between narrative, or narrativity (i.e. the capacity to inspire a narrative response), and medium is of crucial interest. ${ }^{1}$ One key finding of this study is that narratological insights into the organisation, presentation, and mediation of stories cannot be transferred from one medium to another without due modification. Thus, it is hoped that this investigation can contribute to narratology in general, for instance, with regard to the emerging field of transmedial studies that looks at narratives in different forms of expression, communication, and art. $^{2}$ Furthermore, the challenges that the medium of comics poses for narrative theory can potentially shed light on related problems elsewhere, specifically in other forms of visual and multimodal narratives. ${ }^{3}$

\section{Narratology and the Narrative Medium}

From its beginnings in the late 1960s, narratology has developed formal accounts of narrative and its functioning. In the early stages of the discipline, inspired by structuralist linguistics, narratology sought to 
identify the major constitutive elements of narrative. Most importantly, the French literary scholar Gérard Genette defined in his study Narrative Discourse ("Discours du récit: Essai de methode", 1972), based on the analysis of the narrative forms in Marcel Proust's Remembrance of Things Past, the main research issues of narrative analysis, such as narrative time, voice, and mood. In this study, Genette invented several much-used typologies of narrative technique, in particular concerning the narrator, narrative level, focalisation (perspective), and temporal order that will be discussed here in relation to comics.

Today, narratology has been established as a discipline that has both a theoretical and an application-oriented approach to narrative, while still focussing on narrative qua narrative: "What is typical to narratives?", or "What is a narrative?" (Prince 2003b, 3). The various contemporary narratologies, often grouped under the superordinate term of post-classical narratology, ${ }^{4}$ have broadened the field considerably, both with regard to the research questions and the corpus investigated. These changes have pushed the field in new directions and revitalised the theoretisation, research, and analysis of narrative representation, communication, and expression.

The broader approach to narratological problems in recent decades has included, for instance, the question of the reading process, the historical context of narratives and their interpretation, and the history of narrative forms. In part, we can see this development as a reaction to the popularity of transmedial storytelling and story systems, involving large-scale production in various media, such as The Lord of the Rings, the Harry Potter series, Star Wars, and Doctor Who that create a storyworld through multiple documents in various media platforms. ${ }^{5}$ Therefore, narratology can investigate, for instance, the transferability of narratives between various media. This kind of inquiry may ask how the medium affects narrative form and meaning, or how a given medium may be better equipped to carry out some kinds of narrative functions rather than others (Ryan 2004, 34-35).

These changes have been significant for the study of comics. For one thing, the expansion of the narratological field does not just involve 'the corpus argument' - that is, the incorporation of new kinds of narratives into the discipline - but also 'the medium argument', i.e. the question of the applicability of narratology across the media, and the interest in exploring the relation between narrative and the medium. A seminal part of this investigation has also been the applicability of key narratological concepts, such as focalisation, narrator, or voice, across media. In fact, throughout the history of the field, narratologists have frequently tested their concepts, theorems, and analytical approaches with other-than-literary narratives, including comics. The impulse in narratology to conceptualise whatever is general to narrative, irrespective of its various manifestations, has driven research in this field towards intermedial questions and 


\section{Introduction}

comparisons. Many early French narratologists, such as Roland Barthes, Tzvetan Todorov, and Claude Bremond, held that the same story may be realised in different forms of art and communication. For instance, Todorov, who coined the term narratologie in his 1969 study Grammaire $d u$ Décaméron, calling 'narratology' the science of narration (la science $d u$ récit), was interested in developing a theory of narration that could be applied to all domains of narrative, including literary texts, popular tales, myths, films, and dreams $(1969,10)$. Later, in the American context, Seymour Chatman's central works in this field, Story and Discourse (1978) and Coming to Terms (1990), featured a significant amount of reflection on film narratives and their medium-specific ways to tell a story.

A clear shift of emphasis away from literature to other forms of narratives-or narratives across media-is reflected in the Dutch cultural theorist Mieke Bal's works from the late 1970s to the 1990s. Bal's 1977 introduction to narratology, Narratologie (translated as Narratology: Introduction to the Theory of Narrative, 1985), which helped to establish the name of the discipline, analyses in its second and fully revised edition (1997) several visual narratives, from Ken Aptekar's paintings to Steven Spielberg's filmic adaptation of Alice Walker's The Color Purple and the Indian seventh-century bas-relief Arjuna's Penance. Bal's narratological attention to visual storytelling, such as the question of perspective, is an important precedent for the present-day interest in narratives across the arts and the visual media. ${ }^{6}$

During the 1980s, narratology established important inroads in film studies. In the French-language context, notable among the pioneering works in film narratology were Francois Jost and André Gaudreault, who, inspired by Genette's concepts and theorems, sought to develop the theory of film narratives. Their work is directly relevant to the narratology of comics, in particular in relation to questions of perspective and narrative mediacy. In English-language film theory, narratology came into its own in the influential works of David Bordwell, Kristin Thompson, and Edward Branigan. ${ }^{7}$ Today, given its transmedial relevance, for instance, with regard to questions of narrative comprehension, style, agency, temporality, characterisation, and perspective, film narratology is enjoying an increasing influence on the narratological study of visual storytelling in general.

\section{The Beginnings of Comics Narratology}

Narratology has often been applied in comics research, and it has regularly informed comics theory, but no comprehensive narratological theory of comics has been proposed to date. Typically, comics theorists and scholars have made use of narratological notions whenever they have seen it fit for their research question. One rare exception in this regard is Martin Schüwer's Wie Comics erzählen ("How Comics Tell a Story", 
2008), where Schüwer explicitly discusses the relation between narrative theory and comics, and applies Genette's categories of focalisation and narrators to comics. Schüwer's discussion of movement, space, and time is also widely informed by narratological insights into temporality and narrative communication. Furthermore, Schüwer calls for the modification of the existing narratological concepts to better suit the study of comics (2008, 17-26). The Narratology of Comic Art takes up precisely this challenge.

Narratology has also been one of the recurring points of theoretical reference in the French-Belgian 'school' of comics theory since the 1970s, reflected in the works of scholars such as Pierre Fresnault-Deruelle, Philippe Marion, Thierry Groensteen, Harry Morgan, Jan Baetens, and Pascal Lefèvre. In his doctoral dissertation Traces en cases (1993), one of the most cited works in this tradition, Marion adapts narratology creatively, specifically in relation to the notion of narrative enunciation. We will return to Marion's theory in chapters on time, graphic style, and narrative agency. Thierry Groensteen, in his influential studies Système de la bande dessinée (1999) and Bande dessinée et narration (2011), translated into English as The System of Comics (2007) and Comics and Narration (2013), applies certain basic principles of narratology. More precisely, Groensteen makes use of Todorov's principles of narration, succession, and transformation, filtered through André Gaudreault's film narratology $(1999,122,135)$. In the introduction to The System of Comics, however, Groensteen also observes that general semiological theory, with which he associates narratology, has often poorly justified its linguistic bias, i.e. emphasised the verbal component at the expense of pictures and visual meaning. He notes, moreover, that the dogmas of literary narratology are all-too-often applied mechanically to other forms of narrative $(1999,12)$.

Although Groensteen's approach is broadly informed by narratological theoretisation, it does not constitute a narratology of comics. Groensteen characterises his theory with the term 'neo-semiotic', by which he refers not only to the semiotic foundation of his approach but also-with the neo-prefix-to his focus on the language and intelligibility of the medium, or the poiesis of comics, understood in the sense of the conditions of their meaning-making. In a later addition to his neo-semiotic system, Comics and Narration, Groensteen makes the relationship between his approach and narratology somewhat more explicit, especially in his discussion of the narrator concept and subjectivity. Nevertheless, Groensteen reiterates his warning here $(2011,86-87)$ that the extrapolation of narratology, such as the theory of the narrator, to the field of comics studies, will not succeed without due modification and revision of these concepts. ${ }^{8}$

The constant, but also often problematised, references to narratology in the German- and French-language comics theory have been an 
important dimension in the multi-faceted historical relationship between comics studies and narratology so far. Perhaps even more importantly, however, narratology has had an indirect effect on comics theory, also in the English-language research, through general notions of narrative form and technique, for instance concerning voice, perspective, and narrative agency. One particular dimension of this relation is to be found in the introductions to narratology, published before our 'transmedial' era, which have appropriated comics, and especially the short format of the comic strip, to illustrate the theory. These readings are often revelatory of the tendency to make general claims about narratives without paying attention to the relationship between narrative and medium.

Three examples will serve to make the point. First, in Story and Discourse, one of the most influential introductions to narratology in North America, Seymour Chatman analyses at length Frank O’Neal's newspaper strip "Short Ribes" in order to illustrate his theory of the narrative situation $(1978,37-41)$ and, in particular, to explicate the basic narratological distinction between story and discourse in the temporal organisation of a story. For Chatman, the comic strip demonstrates what he calls the process of 'reading out' narratives, that is, how readers may read "the relevant narratives out of or through one sort of nonverbal manifestation" (1978, 41). Most importantly, this means the readers' abstraction of the 'story', that is, what is told, out of 'how' the story is transmitted (i.e. 'discourse'). Second, in their Telling Stories: A Theoretical Analysis of Narrative Fiction (1988), Steven Cohan and Linda M. Shires offer an extended discussion of Cathy Guisewite's comic strip from the long-lived "Cathy" series to illustrate the difference between different categories of plot structure, in particular the so-called kernel and satellite events. This is the distinction, made initially in Story and Discourse, ${ }^{9}$ between events that raise possibilities of succeeding or alternative events (kernels) and those that "amplify or fill in the outline of a sequence by maintaining, retarding, or prolonging the kernel events they accompany or surround" (satellites) (1988, 54). Third, in his 1992 article "Points of Origin: On Focalization in Narrative", Patrick O'Neill develops a theory of focalisation, and especially the notions of embedded focalisation and covert global external focalisation (the implicit perspective of the so-called implied author), through a reading of Bill Watterson's Calvin and Hobbes strip (1985-1995). O'Neill argues that the implied author's covert perspective can be detected in the graphic style of Watterson's strip, that is, in the reality of the characters drawn as cartoon characters.

These three approaches are alike in their use of comic strips as illustrations for the basic tenets of narratology and, at the same time, for their lack of interest in the specificities of the medium. What is problematic, especially, is that in all of these examples, comic strips are perceived, independent of their medium, to represent a narrative in its most 
rudimentary and, at once, most illustrative form. The fact that a comic strip includes pictures, or is structured around panels in a sequence, supposedly has no significance for the way the story is told.

What makes comics so illustrative of the basic tenets of narrative theory? Two immediate explanations come to mind: the visual and succinct form of these strips. In other words, the strip format that tells a simple story in a compact space of a few panels is attractive to use in a textbook, an article, and a course on narratology. Moreover, differences between literature and comics can be neglected since it is presumed that a comic strip is, after all, only a simple narrative for fast and easy consumption. In fact, Seymour Chatman refers to the comic strip as a deliciously edible new form of hermeneutic interpretation: "a glorious new medium" for hermeneutics "to munch on, along with Sunday pancakes" (1978, 41, n16). The discontinuous spatial arrangement of the strip allows Chatman to identify both that which is left out of the images (the 'what' of the story that the reader needs to infer) and the key events of the story. However, the discontinuous spatial form itself, or the visual framing of the perspective, and its partial subjectification as the main character's perspective are not incorporated into the analysis. This is all the more curious since part of Chatman's reading of the strip relies on his notice of a difference between the media: In the comic strip, the distance between the imagined perspective of the panel frame and the character-a king who decides to pawn his crown in order to play at a casino-keeps changing all the while the given angle shifts between panels. In the comic strip, we can look at a character looking at something (that we do not see), get gradually closer to his field of vision (as the perspective alters in each panel), and also literally share part of what the protagonist sees. Perhaps since the differences between literary and visual narratives are too obvious and palpable in this regard, the question of perspective is left aside.

The classical narratologists rarely posed the question of the relationship between narrative and medium even if they were motivated to consider what unites narratives across the media and the arts. Thus, comics remained a transparent medium, too obvious, surely, to deserve any narratological discussion of its own. It is, therefore, high time to think more systematically about the specificities of narrative comics, as well as about the similarities between comics and other narrative media, in narratological terms.

\section{The Narratology of Comics: What Should Be Included?}

As much recent research suggests, comics are increasingly recognised as a medium, or form of art and literature, that is worthy of narratological attention in its own right. One indication of this change is that in the recent decade, narratology has become a frequent component in textbook introductions to the study of comics. ${ }^{10}$ At the same time, there has 


\section{Introduction}

been a growing consent that narratology needs to be taken into new directions through this medium. Several theorists of narrative and comics have recently underscored the importance of such a task. For instance, David Herman notes in his Basic Elements of Narrative (2009a, 74) that "a fully developed narratology of graphic narratives (...) remains a goal for the future". Likewise, in her Contemporary Comics Storytelling (2013), Karin Kukkonen calls for a "comprehensive and coherent narratology of comics" (2013a, 180), and she further explains that such a project would "work toward an understanding of the design and the effects of comics narratives, and thereby contribute to the overall development of narratology" (2013a, 181). Also, Jan Baetens and Hugo Frey state these objectives in The Graphic Novel. An Introduction (2015), where they specify that comics studies, and the study of the graphic novel in particular, can redefine narratology in two significant ways: by showing us that storytelling cannot be separated from its inherent and mediumspecific materiality, and by reintroducing certain aspects of story analysis, such as world-making, space, and characterisation, which have been neglected by the dominant formalist methodologies of narrative analysis.

But what should be included in a comprehensive narratology of comics? What should be its main foci? To return to Kukkonen, for her, a full narratology of comics would address in detail three problematic issues that have been much debated in literary narratology: the story/discourse distinction, the question of the narrator, and focalisation (2013a, 181, 185). To these problems, she then adds the questions of "how comics establish storyworlds and how they characterise and communicate fictional minds" (2013a, 185). In her Reading Bande Dessinée: Critical Approaches to French-language Comic Strip, Ann Miller dedicates a chapter $(2007,103-124)$ to outline those areas of narrative theory that she sees as being particularly relevant for the understanding of narration in comics. Comparing comics storytelling to narrative techniques in film and novels, Miller suggests that relevant narratological questions would involve, in particular, the issues of narrative time; narrative perspective, or focalisation and 'ocularisation' (a character's visual perspective); and narrating instance, concerning issues such as voice and the narrator. Thus, drawing on Genette's distinction between narrative time, mood, ${ }^{11}$ and voice and combining this framework with relevant insights from film narratology, Miller sketches another potential framework for a narratology of comics.

If we were to follow Miller's and Kukkonen's proposals, a comprehensive narratology of comic art should cover the questions of the story/ discourse distinction, the narrator, focalisation, storyworld, and the representation of the characters' speech and thought (or their 'minds'). ${ }^{12} \mathrm{Be}-$ yond deciding upon this set of categories, however, we should consider the relevance of these questions in relation to the capacities and constraints of the medium. For instance, given that time is often represented 
through the spatial organisation in this medium, the premises of the story/ discourse distinction should be subjected to a thorough medium-specific reevaluation. Given also the many ways in which comics tell a story through images, the narratology of comic art needs to cover issues such as narration by visual showing and graphic style, aspects that are simply not relevant in literary narratology. Furthermore, the representation of perspective in comics and the question over the category of the narrator in many ways have more in common with visual forms of narration, such as film, than literature. This means that the narratology of comics is cognizant of developments in film narratology, visual studies, and multimodal studies in this regard. Moreover, as Baetens and Frey point out, the vast topic of characterisation in comics has not attracted the narratological attention that it deserves.

A more comprehensive narratology of comics should not simply borrow its basic concepts, theorems, and approaches from literary or film studies, but it should also critically reflect on those categories, adjust and calibrate them, and, where necessary, invent new ones. Beyond identifying the basic narrative constituents, conventions, and means of the medium, we need to reflect back on narratology to evaluate what this approach allows us to bring into a sharper focus in narrative comics and how the approach should be modified to better achieve that goal. One risk that I wish to avoid is to propose a narratology of comics that would treat the medium as just another form of literature or film, with additional remarks about the function of images in a sequence, or the interplay between word and image.

\section{What Is Narratological Analysis?}

The point of narratology is that it can make relevant claims about the narrative qualities of texts. Narratological concepts are not timeless universals but developed on the basis of particular corpora of narratives that are created in a certain time and place, in a particular form and medium, and for a particular audience. The historicity of narrative forms is also one reason why the division between narratological analysis and interpretation cannot always be very strict. Surely, for many scholars, including myself, narratology is, first of all, a heuristic tool that helps to arrive at a clearer understanding of narrative literature, art, and communication. If we distinguish between narratology and the proper interpretation of narrative texts and works, where the aim of analysis is to relate the narrative text to particular contexts of meaning, narratological analysis can also be seen as a distinct phase in the research process. In this process, the value of narratology is measured in the ways in which it enriches the interpretation and understanding of particular narrative works. ${ }^{13}$ Tom Kindt and Hans-Harald Müller, who seek to make a clear distinction between the narratological process of description and 


\section{0}

interpretation, have argued, more precisely, that narratology serves "as a tool for preparing, initiating, or backing up interpretations; it is understood as a heuristics but not as a theory of interpreting texts" (Kindt 2009, 37).

That said, however, it is important to keep in mind that narratological analysis and description is typically part of an interpretative process, not an objective in its own right. The narratological framework brings forth certain features of texts, works, and documents instead of some others, that is, it focusses on their narrative qualities and their qualities as narrative works. Another problem that we may confront in defining narratology too strictly as a formal description of narrative is that it evokes the thorny issue of form versus content. It goes without saying that narrative forms and devices in comics can be employed to convey meanings, not just to create an artistic form. For instance, the page layout can be used to give a sense of a character's perspective, the frames and the type of panelling can support a theme, and the dynamic between the narrating "I" and the "I" shown in the images can have various consequences for identity and self-image in autobiographical comics. Samuel R. Delany has claimed cogently in this respect that the division between form and content may be a necessary critical fiction, but that it is only a provisional fiction since "at a certain point in the discussion, form begins to function as content-and content often functions as a sign for the implied form with which that content is conventionally dealt" $(1999,259)$. The challenges we face in trying to relate narrative forms to particular meanings, or in trying to understand the relationship between style and meaning, are often highly complex. This complexity, and the way in which form and content work together, is also a major incentive for research in the first place. Narrative techniques can be used for different purposes and to different ends, and they can be successfully connected to a seemingly limitless number of themes, contexts of meaning, and interpretations. ${ }^{14}$

It is important to keep in mind that particular narrative devices, conventions, and strategies in comics always have a history, having developed in particular circumstances of artistic production and reception. For instance, the history of modern comics has been affected by a number of significant changes in artistic production and publication format with various consequences for the way in which stories can be told in this medium. Among such major changes can be counted the inclusion of cartoons and comics in the European nineteenth-century satirical journals, the emergence of American newspaper comic strips and comic magazines in the late nineteenth century, the breakthrough of comics weekly magazines and albums in Europe and comic books in America in the late $1930 \mathrm{~s},{ }^{15}$ the experiments of underground comics in the 1960s, and the development of the graphic novel and independent comics since the 1970s. Many of these transformations have changed conventions 
of storytelling in the medium, such as how characters' thoughts and emotions are presented or how to create a sense of dynamic interaction between action and dialogue, pushing the very limits of the medium. The historical nature of these changes is not my focus here, but I do not pretend that I can ignore it.

We can conclude, then, that narratological analysis is not the same thing as interpretation, but that it requires interpretive skills and its aim is often to aid interpretation. As a heuristic tool in comics scholarship, narratology can be conceptualised as a kind of preliminary stage of interpretation that directs our attention to the narrative features in a given work and helps to analyse and clarify the significance of those features. In Film Narratology (2009), Peter Verstraten is keen to point out that narratology is not a magical solution to all interpretative problems in film analysis. After all, narratology restricts itself to the narrative aspects in films, and it can offer a sound foundation for research only as long as we understand that a more complete narrative analysis is always 'narratology plus X' $(2009,11)$. Verstraten also emphasises that no film-and we might add, no comic-is "unproblematically narrative in its entirety" $(2009,24)$. Even the most prototypical narrative comic, say an action comic or a Tintin adventure, has other dimensions. Comics use symbols and metaphors; they are descriptive, painting-like, lyrical, pedagogical, or abstract rather than narrative; they can define or explain things rather than narrate events; they can put forward claims about a fictional world or our world; they may function as a commentary, an argument, or tell a joke; they can inform or teach us about something; and so on. All this can obviously happen within a larger narrative compositional frame, but it is not always the case. Not everything in comics is narration, and not all comics are narratives. Instructional comics such as guidebooks and instruction leaflets, lyrical comics, or abstract comics do not always tell a story, or their narrativity may be weak. Furthermore, the reading of comics also regularly involves other aspects than the processing of the story and its telling, such as paying attention to the visual features of the image or style and appreciating the narrative drawings or the spatial composition. One of the challenges I will tackle in this book in this respect is the relation between graphic style and narration. Style often serves a narrative function in comics, but its effects and implications are never exhausted by this function.

Narratology may be the best available theory for describing and explaining comics as narratives, but it should not pretend to cover all possible qualities of comics, not even in narrative comics. Therefore, a crucial aspect raised in this book is the judicious application of narratological concepts. This will necessitate a self-critical reflection on the limits and possibilities of these concepts, theorems, and analytical approaches in close relation to a wide range of works. 


\section{Introduction}

\section{The Key Terms}

It is a generally accepted truth in research conducted in the humanities that a clear understanding of the key terms of the research determines the shape of the study and gives it perspective. Thus, some definitions are needed.

\section{Comics}

There is not much use for a strictly formal definition of 'comics' in the narratology of comic art. A formal definition would be, for instance, to state that comics are "the phenomenon of juxtaposing images in a sequence" (Duncan and Smith 2009,3) or "juxtaposed pictorial and other images in a deliberate sequence, intended to convey information and/or produce an aesthetic response in the viewer" (McCloud 1993, 9). We could also state, though less strictly, that "the necessary, if not sufficient, condition required to speak of comics is that the images will be multiple and correlated in some fashion" (Groensteen 2007, 19). The problem with these definitions is not so much that they are too broad or too narrow, but that they qualify comics by reference to some single component that is perceived as the necessary element for something to be called comics. In comics scholarship, the futility of the definitional project has come sharply into view. ${ }^{16}$

The core notion in most contemporary definitions is that comics are juxtaposed pictorial images, a sequence of separate pictures or images, or interdependent images in a series. Groensteen's emphasis on what he calls the foundational order of "iconic solidarity" $(2007,128)$ highlights the idea of the interdependence of images in a series as the most relevant feature of the medium ${ }^{17}$ and distinguishes comics from single images or "unique enclosed images within a profusion of patterns and anecdotes" $(2007,128)$. Such a distinction is indeed quite reasonable - the idea of interdependent images seems crucial to the recognition of something as comics in our contemporary understanding of this word. And yet, it is also clearly problematic. It is problematic, in particular, in the sense that the notion of a "unique enclosed image" is not always perfectly distinguishable from a series of interdependent images, specifically if a single image depicts several events or an unfolding situation. When does a single image become a series, or when can a group of images be perceived as one image? As art history demonstrates to us, a sequence may be embedded in a single image; a single-panel image or painting can comprise a series of images. And, we can find various kinds of image series outside comics, for instance, church paintings, frescoes, or window art that may also conform to this principle. In addition, a formal separation between comics and other related visual media or forms of art, such as picture books, cartoons (single-panel drawing), or animation, is irrelevant here. 
There will always be borderline cases between these forms of expression, such as hybrid formations of picture books and comics (for example, Maurice Sendak's In the Night Kitchen), books illustrated with comics and cartoons (Jeff Kinney's Diary of a Wimpy Kid), or intermedia installations (so-called 'gallery comics'), which render the question of definition difficult or impossible and point out the historically contingent and relative nature of such categorisations.

In the various formal definitions of comics, a lot of ink has also been spilled over the question of the interplay between image and word, and whether it is the defining feature of the medium. The fundamentally hybrid nature of the medium in this respect is also reflected in the frequent attempt in comics theory to reveal a hidden bias in scholarship in favour of either the word (literature) or the image (art, visual culture). By displaying these biases, comics theory has then sought to accommodate the opposite argument, shifting the emphasis again to either literature or visual art and leading to some kind of self-perpetuating dialectic.

For instance, Bart Beaty has identified, in his discussion of the relationship between comics and the art world in the North American context, Comics versus Art (2012), what he calls a 'literary turn', or literary-narrativist prejudice, in the academic study of comics in recent decades. Beaty locates the beginnings of this prejudice in Colton Waugh's The Comics (1947), where Waugh highlighted the importance of certain issues of narrative content, such as continuing characters and narrative form, including sequential images and speech balloons, for all comics $(2012$, 27). More recently, as Beaty claims, a similar bias, which sees comics as popular narrative literature or mass culture but not as a form of visual art, has only strengthened. This is mirrored, he goes on to say, in the coalescence between narrative and literary medium, and the easy slippage between arguments about comics as a narrative form to the discourse of their literariness in academic comics studies $(2012,44)$.

Whenever contemporary comics scholarship has equated narrative form with literariness (the quality of being literary), this is usually justified as an attempt to raise the low cultural status of comics in academia and, in particular, in the field of literary studies. The opposite bias, however, of treating comics as popular visual culture, instead of literature, has also been quite common. For instance, one persistent trend in comics theory, though less common today, has been to see comics as a 'frozen' and 'immobile' form of cinema, or a kind of storyboard for a film that consequently does not require a poetics of its own. ${ }^{18}$ More recently, comics have also been subjected to broader theoretical frameworks of visual language and meaning-making-semiotic, linguistic, or cognitive approaches, for instance, or multimodal studies-in ways that may marginalise the question of comics as a self-respecting medium, literature, or art form.

The literary or narrativist bias in contemporary comics scholarship is a complex issue. First, as most narratologists are happy to admit, there 


\section{Introduction}

is no a priori connection between literary value and narrative form. In literary studies, the concept of literariness-that is, what makes a given work a literary work-is not associated with the question of narrativity but, in the main, with the organisation and style of language that deviates from standard use, and may thus highlight the stylistic choices or the act of expression itself. ${ }^{19}$ Literary value can also be defined through the concept of imagination for instance, but a narrative form is hardly a sufficient requirement for some work to qualify as literature. Second, although much comics theory today tends to see that comics are narratives, it also recognises that there exist many predominantly non-narrative forms of comics, such as abstract, educational, and lyrical comics.

As suggested earlier, the dispute about the biases of comics theory and research can be taken as another reminder of the truly multimodal nature of the medium. For instance, in her Comics and Language (2013), Hannah Miodrag presents almost the opposite argument to Beaty in challenging what she sees as the preoccupation with the visual content in comics studies and criticism. Thus, she undermines the perceptionone that she sees as central in much scholarship in the field-that visual content comes first and needs "to control the narrative and dominate the text" $(2013,11)$. She further concludes that there have been a "general critical reluctance to focus on verbal content" $(2013,18)$ in comics and a widespread "logophobia" in comics studies (i.e. a fear of words subjugating comics' visual form $)^{20}$ in order to insist on the visual value of comics. Miodrag then shows in her insightful analyses how the verbal content in comics, from George Herrimann to Lynda Barry, can be conceived in terms of literary language and aesthetic effect, while she also seeks to problematise, by focussing on linguistic features in comics, what it means for a comic to be read as literature.

We could claim that by conceiving comics as literature, Hannah Miodrag distances the medium from visual art and culture, while Bart Beaty, by making a case for comics as an important form of visual art in its own right, distances comics from the art of storytelling, which he associates closely with literariness, literary value, and literary studies. My focus on narrative comics is an important qualifier here. Comics can be perceived as a form of literature and visual art, but to treat them as narrative also allows us to avoid the word-image dichotomy. I am convinced that there is no particularly good reason to juxtapose the categories of literature, narrative, and visual art in this respect. Comics tell stories by verbal means and by showing images, through their visual and spatial form, through their many combined visual-verbal signs and conventions, and through the interaction of these elements. The communication of a story is, surely, not the only thing that pictures do in comics, or what comics do, but it can also be a focus of its own.

What I wish to emphasise in this regard is mainly that it is quite possible to avoid the circularity of the argument that has characterised 
some recent comics theory: When the pictorial or the verbal character of comics (or the idea of images in succession) has been deemed to be too prominent, the corrective move has shifted the theoretical perspective in favour of the visual component and graphic art, or vice versa. However, there is just so much variety in comics-that is, in works that are produced, recognised as and called 'comics' - in their blendings of images and words, or their emphasis on one or the other, that the interplay between words and images does not provide us with any self-evident starting point for a comprehensive theory of narrative comics. The rich tradition of wordless comics will also always be hard to accommodate within such definitions.

This book will not discuss the issue of word and image interaction as a separate aspect of narrative comics, but it will integrate their interaction in all of its chapters. The focus on comics as narratives allows us to conceive comics in relation to narrative forms, conventions, and strategies, but this does not necessarily make comics more recognisable as literature or visual art. I do think favourably of both of these endeavours since they can open up interesting research perspectives on intermedial and interartistic relations and make us aware of any medium and art-specific biases in our own aesthetic judgements. Furthermore, it can be important to claim literary and artistic values for comics in contexts where serious scholarly interest in this field still needs to be justified. However, it is not the objective of a comprehensive narratology of comics to elevate the value of comics as literature or art, but to study them as narratives and, subsequently, illustrate their value as narratives. It is also to be hoped that the literary and artistic merits of comics need no underscoring.

Therefore, instead of a formal, or even a reasonable, definition of comics, ${ }^{21}$ comics are perceived here in terms of their social institution, that is, the institution of making and reading comics. In other words, the term 'comics' refers here to a medium or, if you like, a form of art and literature that consists of works that are commonly recognised, called, and intended to be read as comics. ${ }^{22}$ The institutional definition enables us to avoid the formalist trap of focussing on a particular structure or device as the distinguishing factor. This approach is not without caveats as there may be some disagreement about what counts as comics today, but the institutional definition suggests that we can appreciate these divergences instead of trying to resolve them according to formal definition.

\section{Narrative and Graphic Narrative}

The question of what constitutes a narrative seems an even thornier issue than what constitutes comics. This is because the phenomena that are commonly recognised as a 'narrative' are incredibly versatile. In the everyday usage of the term, a narrative can not only be an account of events or an act of telling a story, but it may also be equated with fiction, 


\section{Introduction}

ideology, and myth. Sometimes, in comics theory and scholarship, a 'narrative' is used synonymously to the verbal component or the literary aspect of the work, or in the meaning of the story in its verbalised form. These usages are misleading in that narration in comics usually happens both through words and images and, for much of the time, in a conceptual space between them.

One useful distinction for the many dimensions of the term 'narrative' is that it can refer to a product, such as a text, document, and performance; a process, such as narrating, reading, watching, and listening to a narrative; and a mental model in the sense of perceiving something as a narrative. Most contemporary narratological definitions of this term combine these three dimensions in one way or another. A narrative text, a film, or a comic book, for instance, typically implies some conception of who does the telling (in reality and/or fictionally), how the telling is done, and how the audience is supposed to understand the telling (and not just the story that is told). Moreover, the perception of events and occurrences as a narrative is something that can be both an element of the narrative text or document-a character's, narrator's, or an author's perception-and what happens in the reading of some text or documents as narrative.

Narratology tends to perceive certain features as constitutive of narratives. Among these basic properties are included, in particular, the temporal sequencing and the causal connection between the narrated events (or different phases of one event). ${ }^{23}$ However, a chronology of events, a chronicle, does not in itself constitute a narrative, or it makes only a poor story; a causal connection between the events is usually required, related, for instance, through a narrator or an experiencing character, a sense of a world that undergoes changes, a narrative situation (narrator and his or her audience) and a communicative structure (being situated in a specific occasion of telling), or the experiential frame (the author's, the narrator's, or the character's experience and the emotional evaluation of that experience). ${ }^{24}$

Here, I am not seeking a formal definition of narrative since I understand narrative not just as a representation of the time course and causal connections of particular, narrated events, but also as a mental image that, besides being a property of texts and documents or performances, is a common response to kinds of texts in specific contexts. The crucial question here is the narrative quality of comics, comics qua narrative, or what we can also call their narrativity. Storytelling is perhaps the predominant mode of representation, or text type, in comics in general. Therefore, it is useful to think of narrativity in comics as a gradient, a more-or-less quality (see Herman 2009a, 6). Thus, some comics are more prototypically narrative, while the others are less so, for instance, due to the relative predominance of visual description, argumentation, explanation, abstraction, or lyrical expression. 
The term 'graphic narrative' that I sometimes use in this book in lieu of 'comics' is not, strictly speaking, synonymous with the medium of 'comics'. The term has emerged in the recent decade in American academic comics scholarship and literary studies, where it refers to particular types of narratives in the medium. Specifically, the term has been employed in relation to contemporary sustained nonfiction narratives (Chute and DeKoven 2006, 767, 779, n1; Chute 2010, 3). One justification for using this term is the alternative it provides to the now standard but also in some ways problematic category of the 'graphic novel', which is widely used in criticism, publishing, and marketing. ${ }^{25}$ The main problem with the term 'graphic novel' is that many narrative works that are given this label are not fictions at all. Marjane Satrapi's Persepolis, Art Spiegelman's Maus, Alison Bechdel's Fun Home, or Joe Sacco's Palestine are nonfictions in different genres, not novels. As Hilary Chute has pointed out, many nonfictional works in the comics medium "deliberately place stress on official histories and traditional modes of transmitting history" and thus "are deeply invested in their own accuracy and historicity" $(2010,3)$. Therefore, the category of 'novel' is a questionable point of reference. It should also be emphasised that to call some comics graphic narratives is not a value judgement, but simply a descriptive choice. The terms 'graphic novel' and 'graphic narrative' are sometimes used in ways that try to distinguish significant works of comics as literature in contrast to 'mere' comics. The American comics critic Douglas Wolk encapsulates the snobbery of what he calls the "if-it's-deep-it'snot-really-comics" gambit in some reactions to the works of Spiegelman, Satrapi, and Bechdel $(2007,12)$ by readers who do not feel comfortable identifying themselves as comics readers, but prefer to call these works 'graphic narratives'. If I use that term occasionally in this book, there is no snobbery intended.

\section{Medium}

It is a kind of truism in comics scholarship to consider comics as a medium of its own. The problem, again, is that the concept of medium can be defined in various relevant ways. A basic division in the meanings of the term is that a 'medium' can refer, on the one hand, to a channel for transmitting information, such as television, newspaper, film, or the internet, and, on the other hand, to the materials, the technical means and physical instruments of expression, as in the sense of artistic expression. ${ }^{26}$ In addition, a medium can mean those social practices that characterise the use of a particular medium. For instance, media historian Lisa Gitelman defines a medium not only as a technology that enables communication but also as a set of associated "protocols" or social and cultural practices, normative rules, and default conditions that gather and adhere around that technology. The latter involve norms 


\section{Introduction}

and standards about how and where the medium is used, and express a variety of social, economic, and material relationships (2006, 5-7). Conceived in this sense, the comics medium could then mean the ways in which comics are made, published, and read; their reading protocols; and reading culture. Here, 'medium' means in particular a system of communication and information that has a certain material support and is defined by a set of associated social practices, such as conventions of reading, genres, the publication format, and channels of distribution. Finally, different media can also be specified on the basis of their use of one or more semiotic systems (linguistic, visual, auditory, gestural, spatial) to transmit their contents, and which are linked to different approaches to narrative. In this sense, comics is a multimodal, or multichannel, medium that can make a unique contribution to the study of the relation between narrative and medium.

\section{Medium Specificity}

Many of the presupposed medium-specific features of comics have close parallels across media and narrative art forms. Compare, for instance, the function of the gutter-the space in between the panels-and the use of ellipses and gaps of information in written narrative fiction, such as between paragraphs or scene and chapter breaks, acts and scenes in theatre, shot transitions in films, the segmented structure of a narrative mural painting or a fresco, or transitions between lines and stanzas in a poem. Segmented sequences and fragmentation function as a provocation to meaning-making and problem-solving across the arts, inviting the audience to make a connection and fill the gap. Similarly, the alternation between sequential reading and a more global perspective on the composition can play a significant role in reading picture books, and graphic design can create complex relations between words and images comparable to comics. Nor are combined forms of text and image, such as speech balloons, unique to comics, even if comics are famous for them.

To hold a very strict notion of medium specificity can lead narrative theory to focus on an unproductive guarding of borders between the media. However, paying insufficient attention to the question of medium can result in overly facile equations between the presupposed isomorphic qualities across media. One simple alternative, therefore, is to study how comics are, at once, different from and similar to other kinds of narrative media. The stance of this book in this respect is twofold. First, The Narratology of Comic Art questions the idea of hermetic boundaries between comics and other narrative media or arts, especially the closely related forms of visual storytelling, such as single-panel cartoons, picture books, and animation films, on the level of their formal qualities. Second, this book argues nonetheless that certain qualities, conventions 
and strategies, and their combinations are more expected of the medium of comics. The challenge, then, is to explain how the (relative) distinctiveness of certain devices, conventions, and forms of comics can be understood, without seeking some ahistorical essence or formal purity for the medium.

The medium-specificity thesis often has a normative dimension, recommending that artists exploit the distinctive possibilities of the medium and, consequently, define artistic accomplishment and skill on this basis. In its most radical forms, then, the doctrine of medium specificity can contribute to a species of purism. A more relativising understanding of medium specificity, and the one that is sensitive to the historical changes of intermedial relations, holds that media have a range of representational, expressive, and formal capacities, some of which are typical to them, even if subject to appropriation by other media, while they also have other capacities that they share with other media but may put to different uses. For instance, in his discussion of the medium-specificity thesis, the philosopher Noël Carroll has usefully specified "distinctive" to mean two things in this context: the notion that certain of the effects of the given medium are managed both (1) better than other things the medium does and (2) "better than said effects are managed by the media possessed by any other artform” (2008, 36). For Carroll, a 'medium' refers especially to the artistic materials-paint in paintings and sounds in music - and the material instruments, such as a paintbrush or a film camera, used in shaping these materials. Thus, for Carroll, media are the physical media. However, I see no particular reason why the same notion of specificity could not be extended to refer to the uses and effects of the semiotic resources - words, images, and sounds-that characterise a given medium. For instance, many narrative media are capable of creating the impression of a character's inner life, sense perception, and personal voice, but they manage these effects differently, by using and combining different materials and resources such as words, images, sounds, and graphic style. Where one medium may have the advantage over other media and do something better, it may detract in some other way or must find ways to circumvent the challenges posed by the materials, instruments, and resources by which it is characterised.

Medium specificity in this sense, then, is not a determinative category, but a relative and graded one. The notion of 'affordance', derived from environmental psychology and redefined more recently in multimodal research, and which refers to the potentialities and constraints of different resources (or modes) of meaning-making, provides us with one further specification of medium specificity in this sense. In multimodal research, affordance means, more precisely, "the materially, culturally, socially and historically developed ways in which meaning is made with particular semiotic resources" (MODE 2012. Glossary of multimodal terms). ${ }^{27}$ For instance, an affordance of images is the 


\section{Introduction}

capacity to describe visual experience in visual and spatial detail, while in language, it may be relatively easier to convey the idea of changing temporal perspective, negation, or causal relations than in single images. The affordances of a medium are conditioned by those semiotic resources that the medium uses and that it may maximise to a greater effect than other media.

Yet another useful way to conceptualise medium specificity, and one which complements the idea of affordance, is the notion of the constraint. A constraint in this respect can mean a restriction imposed by the material form, the semiotic source, the combination of the material form and the resources, or the publication format. To better illustrate this, let us take two perspectives on the concept of the constraint, one from comics scholarship and the other from the poetics of experimental comics.

In their Power of Comics (2009), Randy Duncan and Matthew $\mathrm{J}$. Smith argue that the comic book is characterised by the following formal constraints:

- $\quad$ spatial limitations (number of pages, page size)

- reproduction technologies (paper quality)

- unrealistic images (comics are two-dimensional and lack the photorealistic qualities of some other forms of visual storytelling media)

- limited capacity to control the reader (readers can view panels and pages in any order and for any duration)

- the page as a unit of composition (allows some control over the reader)

- the conception of images as selected moments

- interdependence of words and pictures

- $\quad$ artistic skill (what the cartoonist is able to achieve)

- the serial aesthetic (most mainstream comic books are published as episodes in an ongoing saga)

(Duncan and Smith 2009, 119-120)

In fact, many of the constraints included here reflect the general capacities of comics as a medium and not just the comic book format. These qualities comprise, in particular, the limited chance to control the order or reading, or the ability to read linearly for the panel sequence and at once freely look at the overall arrangement of the panels. Likewise, the importance of the page layout as a unit of composition, the notion of the panel as a unit of time, the cartoonist's artistic skill, and the interdependence of words and images are common features in the medium across various genres and publication formats. By contrast, the constraints of unrealistic images and the conception of images as "frozen" moments of action are open to discussion. I will revisit these questions later in the book. 
The close connection between a medium-specific constraint and a capacity becomes evident in experimental comics that have used constraints as a means to investigate the basic conventions of the medium. In particular, the activities of the group of French cartoonists and comics theorists known as OuBaPO (Ouvroir de bande dessinée potentielle), founded in 1992, have exposed, by inventing deliberate limitations according to which comics should be made and proposing changes to existing norms and practices, some of the medium's most persistent norms and limitations. ${ }^{28}$ In the first volume of the OuBaPO group's works, Oupus 1, published by L'Association in 1996, Thierry Groensteen enumerated the medium-specific constraints that could be performed on comics and grouped these constraints into two basic categories: generative constraints, which can be employed to produce new works, and transformative constraints, which alter existing works. The original typology included ten generative and seven transformative constraints. ${ }^{29}$

The deliberate generative constraints are as follows:

- Iconic (or iconographic) restriction: The exclusion of specified pictorial elements, such as human figures, from the strip.

- Graphic restriction: The restriction or exclusion of some graphic element or shape, such as a human face or a colour, from the strip.

- Scenic restriction: Constraints of the scene within the strips and the way strips are framed (for instance, every panel is drawn from the same viewpoint).

- Iconic (or iconographic) repetition: The repetition of a single image or sequence of images.

- Multireadability: Comic strips or pages can be read in more than one direction. This has subcategories such as

Acrostic strips: The panels of the comic can be read both horizontally and vertically;

Palindromic strips: The strips can be read both backwards and forwards.

- Reversibility (upside down): A comic that can be read back to front. Famously, Gustave Verbeck invented this technique in his 1903 comic The Upside-Downs Little Lady Lovekins and Old Man Muffaroo.

- Overlapping: The folding of the strip or the page alters the comic, thus revealing a new meaning, story element, or a story. Also, adding elements of other supports, such as a clear acetate sheet, may be used.

- Random order or consecutiveness: Strips whose panels can be read in any order. 


\section{Introduction}

- Regulated distribution: Any pictorial element can be regulated by mathematical, oulippian (i.e. relating to the contraints invented by members of the literary group Oulipo), or other constraints.

- Geometrical arrangement: The layout of the comic strip frames follows predetermined constraints in some geometrical shape. ${ }^{30}$

If we examine in a little more detail how these constraints are conceived, we can see that many of them work with or against certain fundamental norms and expectations about comics. Several concern the spatial form, the principle of sequentiality, and the conventional order of reading. For instance, the repetition of the same pictures, pictorial elements, panels, or sequences makes visible the expectation that individual panels should add something new and valuable to the ongoing sequence and, further, that the diversion from this norm should be motivated, i.e. indicate a significant change in the story content, perspective, or narrative rhythm.

The constraint of reversibility and experiments with random order and geometrical arrangement work with and against expectations about the typical order of reading for comics. One such expectation is that panel relations should be neither random nor mechanical but motivated by the visual composition and the narrative content. Scenic restriction points out that it is expected of comics that the viewpoints keep changing from one panel to another. Other constraints in this category involve the central compositional structure of the panel frame and the way in which the frame is related to perspective in the image. Iconic and graphic constraints, in turn, undermine certain basic expectations concerning picture content. Specifically, these constraints make visible how the use of shapes, such as recognisable characters in a sequence of images, or the depiction of faces increases narrativity in comics.

The constraints in these two lists do not describe the essence of comics, but they manifest many key conventions and expectations. The premise of this book is that only when we create a more comprehensive understanding of the capacities and constraints of comics, together with a better understanding of the shared elements between other narrative media, can we start to modify existing narratology in this field in interesting ways. One great challenge in this sense is that the narratology of comics needs to develop in three different directions at once: through the analysis of the specificities of the medium, the investigation of shared elements across narrative media, and by studying the common properties between related media. The latter involves a kind of mid-level theoretical abstraction between the capacities of the medium and the common qualities and strategies of visual and multimodal narratives. For HansChristian Christiansen, the deep structure of visual storytelling comprises techniques that are "based on general models of storytelling or even on universal human experience", such as facial expressions and postures, relatively independent of style, but to which different forms of 
visual art give different prominence due to their particular substances and constraints $(2000,110)$. Hence, the narratology of comic art needs to develop towards both the specific and the general, not forgetting the shared middle of related visual and multimodal forms of expression.

\section{Choice of Examples}

This book focusses on conceptual issues and is theoretically motivated. As such, the choice of examples is consistent with the need to investigate the medium-specific capacities, constraints, and expectations of storytelling in comics. Individual works in comics have a seminal role in this book as the ultimate testing ground for all theoretical claims. A great number of the examples included here are formally innovative works that explore the possibilities of storytelling and, in many cases, also take issue with their medium, i.e. explore the limitations, affordances, and constraints of the medium. Some examples, from Chris Ware to Fred, go beyond the expressive potential of current practices. Such works, where the narrative medium itself becomes in some way a question, serve particularly well to distinguish major narrative conventions, capacities, and constraints of comic art. However, my main focus is not formal innovation in its own right. Also, many popular comics, from Disney comics to Calvin and Hobbes, are included in the discussion to illustrate how popular comics may explore their affordances and constraints.

Although my focus is on narrative forms and conventions, I do not wish the approach to be wholly synchronic vis-à-vis the history of comics, that is, limited to concerns about the presupposed narrative universals of the medium in a more or less contemporaneous moment of time. Thus, in the course of the discussion, I will make some forays into the history of the medium, including a whole chapter dedicated to the question of the development of narrative forms in 'premodern' comic strips of the early nineteenth-century British satirical journals.

Certain strong trends that have marked the art of comics in the European and North American contexts over the past three decades are reflected in my choice of examples. These trends include, in particular, the independent comics revolution, the emergence of autobiography and other forms of nonfiction comics, the revision of the superhero genre, the influence and predominance of manga, and the development of the graphic novel. The majority of my examples represent European comics traditions and American comics. In the European context, I devote particular attention to Franco-Belgian and British comics, but some examples of Italian and Finnish comics are also included. Most English-language studies in the history, theory, and culture of comics focus on American comic books and comics scene, whereas French comics theory is concentrated on the French-Belgian tradition of the comic album. This book clearly goes against the grain in this sense, developing 


\section{Introduction}

extensive dialogue between Francophone and Anglo-American comics theory and artists. Manga is generally not included in these readings even if Jiro Taniguchi's A Distant Neighborhood has a central role in the discussion of the narrator concept offered here. Note, however, that Taniguchi's graphic novel is a Europeanised and Westernised manga to begin with. Not only is its reading order reversed from the Japanese standard, but also many elements of the visual style in the original Japanese version are closer to Western comics than manga.

Despite the emphasis on longer, sustained narratives in comics, I contend that most of the theoretical claims in this book, exempting questions relating to page layout and the overall appearance on the page, are general enough to be extended to the shorter form of the comic strip. Comic strips have their particular generic outlook, expectations, and narrative poetics that differ, in the main, from longer formats. Perhaps most typically, in conventional gag-a-day comic strips with three or four panels, narrative breakdown is based on a specific effect in the given limited space so that the last panel, or sometimes the penultimate panel, functions as the gag panel. However, serial or continuity strips follow two different kinds of narrative logic at the same time-to be meaningful in three or four panels and as a longer narrative-and, at least since the 1950s, reflecting this double logic and expectation, many strips have been republished as magazines or albums. Therefore, the boundaries between the comic strip and a sustained narrative work in comics are not always that clear-cut, and it is not in the interests of this project to try to enforce that distinction.

\section{Outline of This Book}

The Narratology of Comic Art is divided into five main sections. These sections focus on the sense of time in comics, narrative showing and graphic style, narrative transmission, the presentation of speech and thought, and the relationship between narrative form and the publication format. The 'Time in Comics' chapter of this book examines the issue of temporal organisation in comics by evaluating the basic narratological distinction between story time (the chronological order of the events) and discourse time (the order in which these events are presented in the comic) in this context, and employs the conceptual framework of temporal order, duration (or rhythm), and frequency that derives from that distinction. The discussion of temporal order will develop a more comprehensive understanding of panel relations, the means of connectivity, and the levels of sequencing in comics, thus exploring the ways in which narrative comics exploit the ratio between the compositional units of the panel, sequence, and layout to convey a sense of time. Sequencing in comics is treated here as a multilevel issue, involving the dimensions of chronological, psychological, and presentational sequencing. The 
discussion of narrative rhythm considers rhythmic variation in relation to the narrative units of a panel and a scene, as well as certain mediumspecific techniques that are based on multidirectional panel relations. All these determinants of time in comics are further related to the basic conventions of reading comics that make many of these temporal effects possible in the first place.

The second part of the book consists of three chapters that address the issue of narration through narrative drawings and means of graphic style. Chapter 2, 'Narration as Showing', focusses on the ways in which images tell a story by showing a narrative event, such as some character in action. The fundamental premise of this chapter is that images in comics can visually articulate the narrative by showing certain things in certain ways. More precisely, the concept of 'graphic showing' refers to the visual content of narrative drawing that the image presents for looking, specifically figures and figuration, such as characters and their situations, milieu, or world, which evoke a story-like scenario and thus inspire a narrative response from the readers. This chapter develops one of the fundamental arguments of this book, which is that the development of the narratology of comics requires that we pay attention to the various features of graphic showing that interact with verbal narration and perspective (focalisation). Chapter 3, 'Character as a Means of Narrative Continuity', develops the notion of narration by showing further by examining the way in which the repeated character figures, in an ongoing situation or action, function as a basic sequential model and a tool of narrative continuity. The notion of salience is especially helpful in conceiving the characters' function in this way. Narrative salience refers to the importance of how something is shown, and how certain elements, in particular relating to characters and their actions, are significant for an understanding of the narrative as a coherent whole. In this regard, theories developed in film studies that stress the importance of characters' spatio-temporal paths, and how those paths can function as spatio-temporal attachment and subjective access for the viewers, have much to offer for a narratological understanding of comics. Chapter 4, 'Graphic Style, Subjectivity, and Narration', discusses the narrative function of graphic style in channelling story information. In particular, this chapter concentrates on two specific narrative functions of style in comics-stylistic variation and rupture-as means of presenting the characters' subjectivity, i.e. their thoughts, perceptions, and emotions. The concept of 'mind style' also allows us to rethink the relation between graphic style and narration with regard to the presentation of a character's mind. Furthermore, the chapter argues that wordless comics have the unique potential to undermine the ascription of states of mind or perception to characters since it may be difficult to know the degree of subjectivity of vision from images alone. 


\section{Introduction}

The third part of the book focusses on narrative transmission and mediacy, i.e. the storytelling process that mediates between a narrative comic and the reader. Chapter 5, 'Narrative Agency', addresses the question of narratorial authority and enunciation through a case study of Jiro Taniguchi's graphic novel, A Distant Neighborhood, and examines the reasons why it is challenging to describe the source of narration in such works. By 'narrative agency', I mean the conceptualisation of a kind of global frame of narration that enables the reader to estimate the meaning and importance of the various visual and verbal or visual-verbal elements, their relations, and their alternating perspectives at the micro-level of the story. The chapter will start with a comprehensive discussion of the narrator concept. Chapter 6, 'Focalisation in Comics', develops the study of perspective in this multimodal medium, by examining the distinction and interplay between perceptual and cognitive focus, the interaction between various simultaneous perspectives, as well as the relation between verbal narration and graphic style. More precisely, the chapter investigates certain hitherto little-studied aspects of perspective-taking in comics: the relationship between narrative voice, mode, and showing; the epistemic access to the point of perception and of what is perceived, concerning the spatially explicit (or determined) point of perception in graphic images; the distinction between perceptual and cognitive focalisation; the issues of embedded and simultaneous focalisation in a literal sense, i.e. the simultaneity of different visual focalisers both within and without the image frame; and the complex scale of intermediate (visual) focal points between internal and non-character-bound positions, or between personal and impersonal viewpoints. Chapter 7, 'Characterisation in Comics', addresses how characters are presented in comics and develops the issue through a sustained analysis of two adaptations of Dante Alighieri's Inferno. The chapter will elaborate on how comics prompt their readers to ascribe properties, or traits, to their characters, that is, how the reader's construction of a character is cued in the elements of the story. The chapter focusses on the main parameters of characterisation in comics, in particular, with regard to the characters' mimetic (realist) function, i.e. how they can be perceived as possible persons. Moreover, the chapter will discuss the ways in which characters can achieve a sense of psychological or conceptual complexity in comics, emphasising the significance of types and caricature in the history of the medium.

The fourth part of the book focusses on speech and thought representation and the narrative function of dialogue. Chapter 8, 'Presenting Minds in Comics', discusses three central issues in the presentation of characters' (or the narrator's) minds in comics: the mimetic aspect of the image, the problem of free indirect discourse, and the interaction between visual focalisation and verbal narration in first-person narration. The theoretical discussion will be illustrated by representative extracts 
from comics, moving from third-person to first-person narration, both in fiction and nonfiction. The analysis of these examples shows that graphic narration subjects to doubt some of the theoretical presuppositions, prevalent in much narrative theory that is based on literary narrative fiction, and requires us to critically examine various key distinctions, such as those between first- and third-person narration; between direct, indirect, or free indirect discourse; and between telling and showing. Chapter 9, 'Dialogue in Comics: Medium-Specific Features and Basic Narrative Functions', focusses on the dialogue form in comics as a key narrative device and examines the elements and narrative functions that characterise conversational scenes in this art. The multimodal character of conversational exchange in comics requires that we attend to the interaction between the utterance and the elements of the narrative drawing, that is, the ways in which the dialogue form (as written and drawn speech) interacts with what is shown in the image. Crucial aspects in conversational scenes in comics are facial expressions, gestures, body language and shape, and participant involvement. Equally, the expressive functions of typography and pictorial symbols, onomatopoeia, as well as graphic style, panel framing, and page layout, can play a major role in creating conversational scenes. The chapter is structured around four questions: the embodied speech situation in comics, the bond between the speaker and the utterance, the temporal and rhythmic functions of speech balloons, and the narrative function of visual and verbal contrast in dialogue.

The last part of the book discusses the relationship between narrative form and the publication format. Chapter 10, 'Picture Story and Narrative Organisation in Early Nineteenth-Century British Caricature and Comic Strips', is a study in diachronic narratology that investigates the basic narrative techniques and operative principles of juxtaposition, sequentiality, and simultaneity in the historical context of the so-called British Golden Age of Caricature (1780-1820) and the caricature magazines published between 1825 and 1835. This investigation will specifically examine the conception of panel arrangement and panel relations, illustrating how the early cartoonists experimented with the principles of juxtaposition, sequentiality, and simultaneity in response to the demands of the publication format, thus contributing significantly to the gradual emergence of the modern comic strip.

\section{Notes}

1 Mary-Laure Ryan argues that while a narrative is a "semiotic object", narrativity refers to the quality of "being able to inspire a narrative response" (2006, 10-11). See also Wolf (2003).

2 See Ryan (2004, 1-40, 2006, 4-7).

3 Kress and van Leeuwen define a multimodal document as "any text whose meanings are realised through more than one semiotic code" $(2006,177)$. See also Stöckl (2004). 


\section{Introduction}

4 See David Herman's definition: "Postclassical narratology [...] contains classical narratology as one of its 'moments' but is marked by a profusion of new methodologies and research hypotheses; the result is a host of new perspectives on the forms and functions of narrative itself" $(1999,2-3)$.

5 See Ryan (2005, 2013), Ryan and Thon (2014, 12-13), Jenkins (2006, 195-196) for the definition of transmedial storytelling.

6 Writing about the contribution that narratology can make in visual studies, Bal emphasises that "the analysis cannot be limited to the application of narratological concepts to visual representations ('How do images tell?')" $(1990,744)$. Rather, she claims, "the confrontation between the narratological apparatus and the visual image inevitably changes or even subverts the categories" (ibid.).

7 See, for instance, Bordwell (1985) and Branigan (1984, 1992).

8 In his 1988 article "Narration as Supplement" (2014, 179), Groensteen also "dreams" of founding a narratology that would be specific to comics.

9 Chatman develops this distinction on the basis of Roland Barthes's notions of nuclei and catalysers, explained in "Introduction to the Structural Analysis of Narrative", in Image Music Text. Trans. Stephen Heath. New York: Hill and Wang, 1977, 79-124.

10 Such narratologically informed textbooks include, for instance, Saraceni (2003), Miller (2007), Kukkonen (2013b), and Postema (2013).

11 For Genette, the narrative mood concerns techniques of filtering the narrative, such as focalisation and techniques of speech and thought representation $(1980,161-211)$.

12 Steven Surdiacourt also proposes a model of comics narratology in his $\mathrm{PhD}$ dissertation (2015), divided into the issues of segmentivity/sequentiality/ structure, focalisation, and narrative voice.

13 I side with Marc Singer, for whom "putting narrative theory into practice is primarily a matter of conducting an informed, close reading of the text, looking at all the ways the narrative elements contribute to its meaning" (2012, 59).

14 For instance, as much research shows, forms of speech and thought representation, such as free indirect discourse, can realise a multitude of functions in the nineteenth-century novel (McHale 1978, 274-284, 1993, 60-62; Cohn 1999, 163-180).

15 See, for instance, Jared Gardner, on how the popularity of the comic book raised the question of page composition to a new level, both in terms of a new kind of visual whole and narrative breakdown (2013, 249-250).

16 See, for instance, Groensteen (1999), Meskin (2007, 376), Hatfield (2010, 5), and Beaty (2012, 31-44). Samuel R. Delany has argued that instead of a definition, we need a "careful, analytic description of what is vital, intriguing, newly noticed, and wondrous about comics (what they are; how they work)" (1999, 245).

17 For Groensteen, iconic solidarity means "interdependent images that, participating in a series, present the double characteristic of being separated-this definition dismisses unique disclosed images within a profusion of patterns or anecdotes-and which are plastically and semantically overdetermined by the fact of their coexistence in praesentia" $(2007,17)$.

18 For instance, Jules Feiffer argues $(1965,68)$ that "comics are movies on paper-the final dream!". Elisabeth Potsch and Robert F. William voice the same view: "Comics is cinema without motion or sound" $(2012,13)$.

19 See also Miodrag, who points out that literary quality (literariness) in comics has much less to do with the ability to tell a story than using language in particular self-reflexive and poetic ways $(2013,18)$. 
20 Miodrag derives the term logophobia from Dylan Horrocks, who argues that, for Scott McCloud, it is "as if the very presence of words-any wordsin a comic is a potential threat to its identity as a comic. To protect that identity, it is essential for the pictures to dominate the words" $(2001,5)$.

21 Groensteen conceives his definition of comics as "reasonable", i.e. one that pertains not to all comics, but "the totality of historical manifestations of the medium" (2007, 17).

22 Beaty argues, similarly, for an institutional definition of comics (2012, 31-44). A social and institutional definition of comics is also used in Hague (2014, 16-18) and Barker (1989, 8).

23 For Gerald Prince, for instance, a narrative is "the representation (as product and process, object and act, structure and structuration) of one or more real or fictive events communicated by one, two, or several (more or less overt) narrators to one, two, or several (more or less overt) narratees" (2003a, 58).

24 Following Monika Fludernik's definition of the term "experientiality", i.e. the communication of anthropomorphic experience $(1996,30)$.

25 The term has refered to different kinds of long-form comics since Will Eisner called his A Contract with God (1978) a "graphic novel". For the debate over this term, see Labio (2011, 125-126) and Hatfield (2005).

26 See Ryan (2004, 15-20) and Carroll $(2008,35)$.

27 The notion of affordance derives from James J. Gibson's interactionist ecological theory, where an affordance is a way of perceiving a given environment in relation to what the various elements of that environment afford one to do-'one' referring here to both people and animals (1979). The notion has been widely applied in the study of multimodality since Günther Kress adopted the term (2010). See also Kukkonen (2013b, 167).

28 The group's cartoonists have included, for instance, François Ayroles, Anne Baraou, Gilles Ciment, Patrice Killoffer, Jean-Christophe Menu, and Lewis Trondheim.

29 The transformative constraints include the means of manipulating an already-existing comic by expansion, reduction, substitution graphic reinterpretation, reframing, and other techniques.

30 I am using Brad Brooks's translation of these constraints, and some of his wording in the definition of the terms. Please see http://www.paolacarbone. com/cultura/2011/oubapo.pdf. Jacques Dürrenmatt claims that the two essential constraints in the medium are the page layout and the constraints that the writer and the cartoonist, even when they are the same person, impose on each other $(2013,129)$. He distinguishes these from stylistic constraints (the expectation of stylistic unity) and self-imposed constraints, such as the OuBaPo activities. 
This page intentionally left blank 
Part I

Time in Comics 
This page intentionally left blank 


\section{Time in Comics}

The opposition between erzählte Zeit (story time) and Erzäblzeit (narrative time) (...) is less relevant perhaps in other forms of narrative expression, such as the roman-photo or the comic strip (or a pictorial strip, like the predella of Urbino, or an embroidered strip, like the "tapestry" of Queen Matilda), which, while making up sequences of images and thus requiring a successive or diachronic reading, also lend themselves to, and even invite, a kind of global and synchronic look-or at least a look whose direction is no longer determined by the sequence of images.

Gérard Genette (1980, 33-34)

It is a kind of commonplace in comics studies to argue that space and time are intricately intertwined in this medium. ${ }^{1}$ However, the discussion of the spatialised forms of temporality in comics has often remained rather one-sided. It has been common, for instance, to privilege particular elements of the composition, such as the space between the panels or the panel as a unit of time, to illustrate how comics prompt readers to make inferences about temporal progression. In this regard, narratology can provide a more comprehensive account of time in narrative comics, and it can also offer a precise vocabulary and a widely tested approach to this topic. Specifically, the narratological distinction between story-time (the chronological order of the events) and discourse-time (the order in which these events are presented in the comic) allows us to investigate the basic forms of temporal structure in comics. While parts of this model have been applied in earlier comics studies, much of its potential remains to be explored.

Sometimes comics explicitly reflect on the capacities that affect the communication of temporal meanings in their spatial composition. Chris Ware's graphic novel Building Stories (2012), a boxed set of fourteen different kinds of printed works in comics, investigates the relationship between story-time and discourse-time at various levels, thus ambiguating the temporal and chronological relationship between various parts of the story. Building Stories poses the question of narrative memory by allowing the reader to "build a story" in any possible order of its parts. 
The navigational mock directions given on the back of the box further encourage the sense of freedom and randomness in this regard. They indicate, in a kind of ironical version of the method of loci, places in any "average well-appointed home" where one can "set down, forget or completely lose" any number of the parts of the composition. ${ }^{2}$ Thus, the title of the work itself can be understood in its literal sense as stories about buildings, stories told by buildings-indeed, the Chicagoan tenement house that is central to the story actually has a narrative voiceor stories told in relation to buildings and memories about their spaces (the method of loci). There is also the metaphorical meaning of the building blocks of a story - the toy set, the game board, or the model kit analogy - with which the reader can creatively engage in order to create a story within some temporal frame.

Building Stories sets the different printed materials that it comprises in a circular network of complementing units, thus suggesting the idea of a story that needs to be processed through a web of interconnected stories rather than conceived in a strict linear order. All the different story units have the potential to serve as background memory to be used for filling in the blanks created by the other parts. The order of the reading can always be reversed if desired or needed. This freedom highlights the importance of something that the readers conventionally do with narratives regardless of the medium: establish the chronology of their events, identify the characters, construe their personalities and relationships, and make note of the significance of the milieu and the circumstances. Thereby, Building Stories extends the manipulation of the order of the events from the level of presentation to the order of reading.

That said, however, it must be stressed that the temporal openendedness of Building Stories concerns primarily the order of reading the various printed works rather than the sense of chronology in the story. The story-time is not random even if the temporal relations between the different instalments are not clearly defined. How is this possible? First, the reader can make inferences about the order of the events on the basis of the image contents pertaining, for example, to signs of aging in the reoccurring characters, their changing relationships, homes, and milieu. Furthermore, we can detect indications of time in the depiction of the protagonist's thoughts and experiences (how they develop and change, or imply earlier or foreshadow future events). The main storyline involves a young woman with a prosthetic leg, a former art student and later a florist, whose life the reader may follow for about a decade, with some flashbacks to her childhood, and intersecting stories about the people who lived in her tenement house in Chicago when she was in her twenties. Later, we follow her life as a mother in a suburban home. Second, one of the instalments, the hardcover book with a gilded spine that is modelled after America's Little Golden Books for children, functions as a kind of aid for the construction of a temporal continuum. 


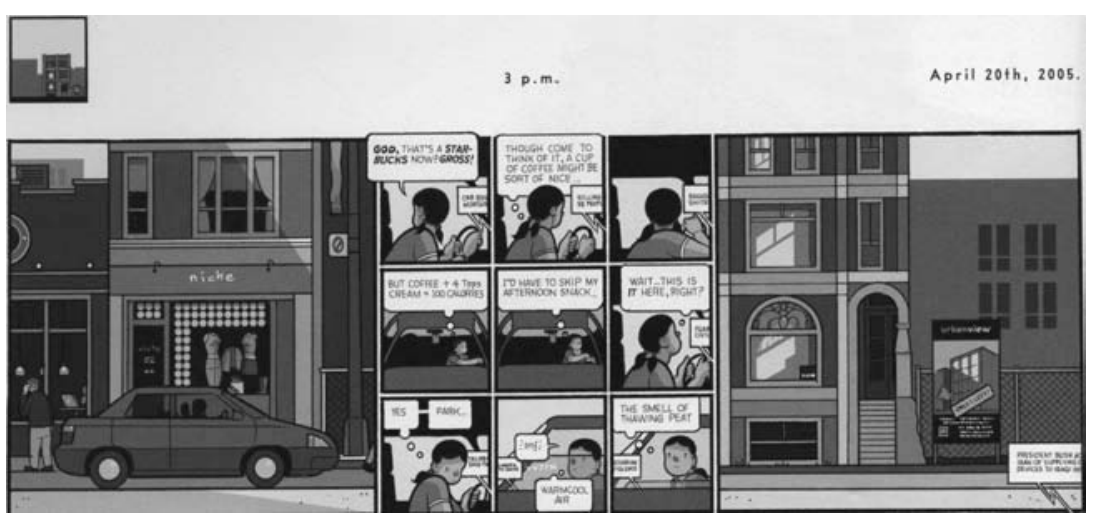

Figure 1.1 “April 20th, 2005.” Building Stories (2012) @ Chris Ware.

The book gives an exact date, 23 September 2000, for a one-day story in a three-floor Chicago apartment building. Consequently, this date, which is also the title of the instalment, and the flash-forward of the last page in this instalment (20 April 2005), may be used to determine the order of the other events in the other instalments, where there is no such precision, or at least assess what might be the most coherent arrangements in this regard (Figure 1.1).

It is possible to read Building Stories as a network of events and memories to be found and links to be made, and perhaps again to be remade as other instalments offer new information. Yet, there is also much support for a chronological understanding of the events. Thus, chronological order and the network of interconnected moments are not opposite temporal structures, but they can be conceived of as complementary options and in terms of their interaction. Building Stories both undermines and reinforces the distinction between the order of the events in the story and the order of their presentation. The readers need to be particularly active in creating a sense of chronology out of the seemingly interconnected moments.

In what follows, this chapter will evaluate the relevance of the fundamental narratological distinction between story-time (the order of the events) and discourse-time (the order of their presentation) in the context of narrative comics. However, this framework needs to be related to the conventions and expectations of reading comics, such as the convention of a kind of synchronic look that Genette mentions above. It is not merely a matter of formal analysis. Also, Building Stories highlights the significance of these conventions through its great variety of shapes and formats of comics, from pamphlets and tabloid-size magazines to cloth-bound books. 


\section{Story and Discourse in Narrative Comics}

The temporal structure of narratives has been among the most central questions of narratology throughout the history of this discipline. ${ }^{3}$ The key distinction in this respect is between story-time, meaning the temporal order or succession of the events in the story, and discourse-time (or narrative time), in the sense of how the events and the story contents are arranged and presented. Narratology has not invented this distinction, but has systematised its study, providing us with a much-tested approach for analysing temporal organisation in narratives on that basis. ${ }^{4}$

However, what complicates the matter is that the scope of this distinction has always been under some debate. ${ }^{5}$ The Genettean understanding of 'discourse' comprises, besides narrative time, the entire expression plane of narrative mood and voice. Narrative mood refers to the regulation of narrative information through distance from the things that are told, such as by means of perspective, while narrative voice means the act of narrating through a narrative situation, in particular by a narrator. Other broader definitions contend that 'discourse' comprises elements of style or what is specific to a medium. Similarly, the 'story' (the 'what' that is narrated) is not always limited to the chronological order of the recounted events of the story, but it may refer to the basic elements of the story content, in particular the characters and their world.

The problems that result from maintaining the broadest definitions of these two terms have not remained unnoticed by narratologists who have turned their attention to visual narratives. There are, at least, two main objections that we can level against the broad definition of discourse in this context.

First of these is the difficulty in differentiating between graphic style, or the materiality of the image, and the narrative meaning of the images. Martin Schüwer has problematised the usefulness of the story-discourse distinction on this basis $(2008,23)$, by arguing that this divide cannot be as clear-cut in comics as it may be in the linguistic structures of verbal narratives. The second challenge is that posed by Genette: the global or synchronic look, or what is called "tabular" reading in reference to a tableau (picture, painting, table), originally defined by Pierre Fresnault-Deruelle (1976). More precisely, the notion of tabular reading refers to features in comics that invite a nonlinear, or not only sequential, reading of the panels and where, thus, the whole of the spatial arrangement merits a more global look and appreciation. There are great differences between comics in this respect. For instance, certain "exploded" scenes that are typical of Guido Crépax's Valentina invite a pronouncedly tabular reading: the panels on the page are not integrated into a logical continuum in terms of a sequence, but these reflect the protagonist's mental state through relations of contiguity that are sometimes quite complex (see Fresnault-Deruelle 1976, 23). Here, the composition invites 
a synchronic look at the whole as a unit of graphic design and narration. Such arrangements defy the story-discourse distinction: is there any sense of temporal order in scenes that focus on the protagonist's mental state or the various perspectives of the situation at hand?

Schüwer's advice, which I follow here, is to restrict the application of this distinction to the study of temporal structure. ${ }^{6}$ The limitation will be beneficial for our analysis of perspective (focalisation), voice (narrative mediation, the narrator), characterisation, and style in later sections of this book. The issues of mood and voice will be discussed, independently of the story and discourse divide, in chapters dedicated to narrative agency, style, perspective, and the presentation of speech and thought.

\section{Order, Duration, and Frequency}

Gérard Genette systematised the distinction between story-time and discourse-time, based on the distinction between fabula and syuzhet in Russian formalism, by proposing to study this relation through three essential determinants of time: order, duration, and frequency $(1980,35)$. In the following, I will concentrate, in particular, on the questions of order and duration in comics storytelling. In the course of this discussion, I will also examine a number of important theories of time and space in comics studies, evaluating especially their narratological relevance.

\section{Temporal Order}

The narratological analysis of time focusses on "anachronous" sequences, or anachronies that depart from the sense of narrative present that is established in the given narrative ${ }^{7}$ and thus involve some shift of balance between the levels of story-time and discourse-time. The basic categories of asynchrony in this regard are retrospection (flashback), anticipation (flashforward), and the lack of temporal chronology, or what Genette calls 'achronism?. ${ }^{8}$ Retrospection involves the narration of past events in relation to the narrative present, and anticipation involves the narration of future events in relation to the narrative present $(1980,40)$. Such anachronies can be more or less explicit, or take multiple (or embedded) forms so that, for instance, an anticipatory passage includes retrospection or a flashback includes further flashbacks $(1980,79)$. As a case of 'achronism', Genette treats certain passages in Marcel Proust's In Search of Lost Time, where the told events are cut loose from any temporal situation, or their relation is random in this respect. For instance, the succession of train stations that are described at the end of Sodome et Gomorrhe evokes in the narrator's mind a series of stories from different times, only connected by the same space or a theme. Similar to stories 
that are connected through the same building in Building Stories, memories are thus organised according to a sequence of places, or spatial contiguity, rather than temporal continuity.

In narrative comics, anachronies can be identified in any significant changes with regard to the progress of time in the story world. Temporal gaps are perhaps the most obvious starting point for the analysis. The gaps, and other types of temporal shifts such as flashbacks, can take place between formal elements of the composition, such as panels, strips or tiers of panels, pages, and double spreads, or between narrative units, such as passages, scenes, ${ }^{9}$ chapters, and instalments. The following discussion of temporal order will proceed in three steps by looking at the temporal function of the panel relation, the means of connectivity between the panels, and the levels of sequencing.

\section{Temporality in Panel Relations}

Perhaps the most obvious element for manipulating temporal order in comics is the panel relations that invite the reader to construct meaningful connections and fill in the gaps in information. The transition between two panels does not necessarily indicate a temporal shift-a sequence of panels may, for instance, depict the same character or object at the same time from different angles-but temporal transitions between panels are so common in the medium that they may amount to a kind of default expectation. Notice how in this wordless page in Bastien Vivès's graphic novel A Taste of Chlorine (2011, Le Goût du chlore, 2008), which depicts the glass ceiling of a swimming pool in several panels from a character's subjective perspective, the content of the images stays nearly the same, but the relation between the point of perception and the object of perception changes (Figure 1.2). The perspectival changes indicate slow temporal progression, relating to the swimmer's sense of movement on his back in the pool.

Some of the most popular theories of meaning-making in comics focus on the gaps in information between the panels in a sequence. In these approaches, the missing information in the 'gutter', the space between the panels that can have a literal form (an empty space) or remain virtual (a mere frame separating two panels, or frameless panels, for instance), involves at the same time a distinctive formal feature of the medium, a convention of reading, and mental activity. In Understanding Comics, Scott McCloud famously referred to the mental completion of gaps between panels as 'closure'. Closure, McCloud specifies, is "the phenomenon of observing the parts but perceiving the whole" (1993, 63), that is, the mental activity of completing the missing information between the panels to make a meaningful whole, or to use McCloud's wording, to "mentally construct a continuous, unified reality" $(1993,67)$. The idea of gaps in information, which give rise to meaning in the reading process 

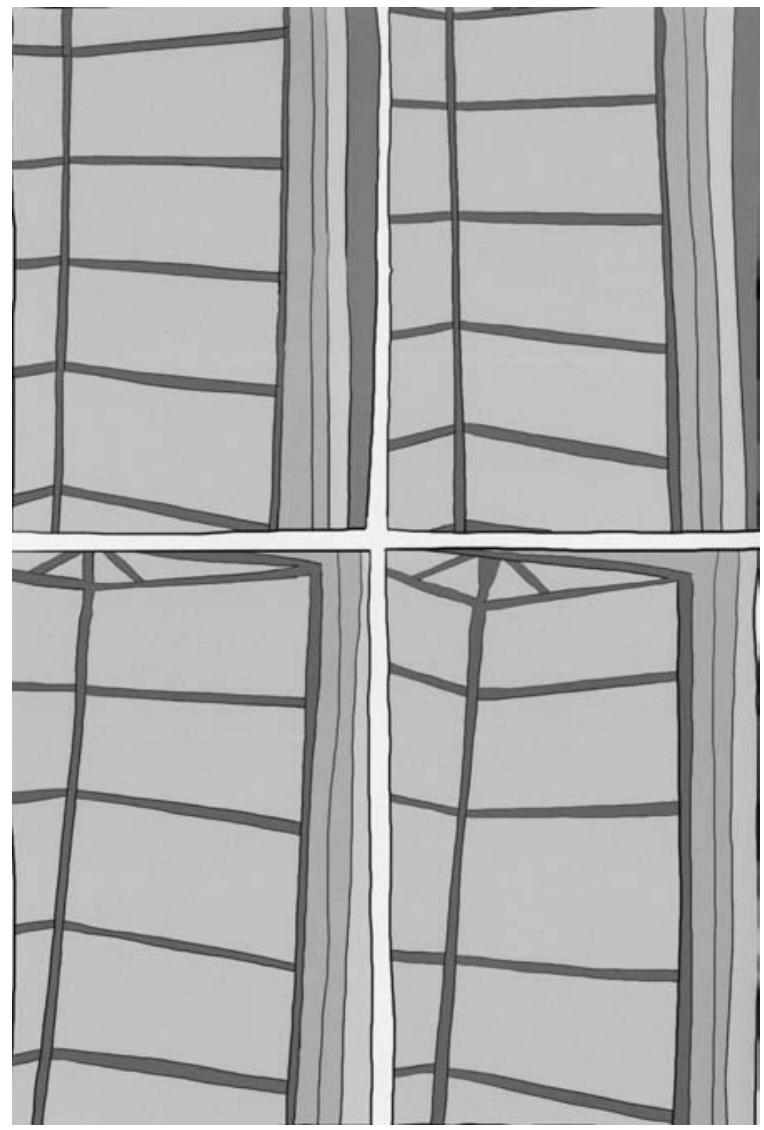

Figure 1.2 Extrait de l'ouvrage. A Taste of Chlorine (2011). Bastien Vivès (C) Casterman. Avec l'aimable autorisation des auteurs et des Editions Casterman.

by provoking the readers to make connections, is fundamental to comics. When two images or pieces of information are juxtaposed in space, very little seems to be needed for the presentation to suggest a meaning and, perhaps, a story. McCloud's choice of the term, however, is confusing in the context of narrative theory, where 'closure' has an established meaning as "the satisfaction of expectations and the answering of questions raised over the course of any narrative" (Abbott 2005, 65-66). Here, in other words, closure refers to an outcome in a narrative, and not to all gaps of information along the narrative.

Yet, from our narratological perspective, the terminological confusion is perhaps less crucial than the way in which the closure theory limits the question of panel relations, and consequently the question of time, to a 
linear connection between two subsequent panels. As a side product of this theory, the space between the panels, the gutter, is reified as a kind of essence of the medium. ${ }^{10}$ In this respect, Jan Baetens and Hugo Frey's critique of what they see as the "inflated" value of the gutter concept is helpful in two important ways. First, Baetens and Frey point out that the diegetic function of the gutter can vary widely:

in certain cases, it [the gutter] may be utterly unremarkable (in the two meanings of the words: unnoticed and also not worth noting); in other cases, its role can be paramount (and of course, the same remark applies to the visual, pictorial function of the gutter, which is by all means a key aspect of page composition).

Thus, we must recognise the importance of the panel relation, not the space in between. Second, in their critique of the closure theory, Baetens and Frey point out that panels in comics, at least in longer works such as the graphic novel, are structured on various levels simultaneously, including the strip or tier, which can be organised horizontally, vertically, or as a combination of both; the page, which can have a wide variety of sizes and formats; and the book, where the size and format can also diverge widely $(2015,104-5)$. To these three levels, we could further add forms of layout and panel organisation that go beyond the structure of the strip and the grid, such as the impression of depth through stacked or superimposed panels and the directionality of the strip/tier (see Bateman et al. 2016), or the treatment of the whole page as a geometrical shape. Consequently, the contact between the panels can, on all these levels, be either sequential or more autonomous, that is, have a painterly function, or prompt a global look at the composition as a whole.

Let us consider the manipulation of temporal order through some panel relations in Nicolas de Crécy's wordless comic album Prosopopus (2003). The 'mute' quality of the story creates certain representational pressures in its own right and promotes an associative mode of visual reading that prompts the readers to pay attention to the breakdown of the story on the visual plane alone. ${ }^{11}$ Beyond attending to the panel relations, juxtapositions, and transitions, this also involves inferences about changes in viewpoint, and paying careful attention to various visual cues, such as facial features, gestures, clothes, and details of the objects. Specific 'time props' and time cues are also highlighted. These include, for instance, empty panels that indicate the duration of time, symbols like numbers or the clock face, pictures within pictures, or changing ratios of light and shadow, details in the landscape, or changing seasons.

Part of the challenge in reading Prosopopus and understanding the chronology of its events is due to the effects of juxtaposition and montage between the panels. Events and scenes that are spatially and temporarily 
removed from each other can follow each other without any explanatory frame. What is particularly remarkable in this respect are larger intrapictorial relations, including tiers of panels, a page or a double-page setup, or panel-to-panel references throughout the whole album, that may convey information about temporal relations through repetition, visual analogy, or other meaningful correspondences between the panels and their sequences. At times, De Crécy employs an analogous technique to the cinematic match cut to indicate a simultaneous change of scene and/or temporal frame: transitional panels that lead from one scene or temporal frame to another through some visual detail in the image (a spot on the floor, a tile on the wall, hands holding a tool/weapon, fragment of a paintings, and so on), or the effect of zooming into a detail that 'connects' somewhere else, thereby establishing a graphic match between the panels. In some panels, by contrast, the depiction of a video camera viewpoint, supposedly held by the story's namesake, the monster Prosopopus, helps to create the narrative effect of condensed layers of time. On a page towards the end of the story, one flashback follows another, including the scene of assassination seen in the beginning of the story. Here, the panels of the earlier scene are marked off by two (nearly) blank panels, while the sequence is at the same time sped up by diminishing and narrowing the size of the panels. This is a flashback of a flashback that, we may assume, takes place in the main character's mind.

Prosopopus illustrates to us how non-verbal information alone can serve to construct complex temporal layers and relations in the story and, thus, require that the reader engages in a lot of back-and-forth checking of story elements beyond the immediate panel sequence. Moreover, it makes manifest the significance of the narrative context, such as a passage or scene, which allows us to determine the temporal or other meaning of a given panel transition. The narratological potential of McCloud's six types of transition between the panels-moment-tomoment, action-to-action, subject-to-subject, scene-to-scene, aspect-toaspect, and non-sequitur (serving no narrative purpose) transitions-is compromised by the fact that the typology does not take the context into consideration. Furthermore, the categories describe panel relations at varying levels of organisation and meaning-making. As Bart Beaty has pointed out, the category of scene-to-scene transition actually describes a transition between larger units of narrative, that is, scenes of narrative, rather than just between two subsequent panels. The other types of transition reflect more micro-level shifts of temporal frame, perspective, subject matter, or causal connections. In contrast, the non-sequitur transition involves a non-narrative relation between panels, such as a challenge to narrative logic (Beaty 1999, 70). ${ }^{12}$

At the same time, McCloud's understanding of the function of panel transitions does not capture the full flexibility, instability, and heterogeneity of their functions. Still other types include changes of narrative 
voice and mood, such as perspectival shifts between characters, or altering effects of angle, focus, and objectivity and subjectivity in the viewpoint. ${ }^{13}$ One rather common type of transition is the change of truth-value (modality in the linguistic sense) with regard to the image content in the panels. The modality-to-modality transition, involving a transition in the truth-value or credibility of what is seen, for instance, in a dream, fantasy, hallucination, or memory sequence, is regularly accompanied by stylistic markers, such as changes in the graphic line, lettering, and colour, or alterations in verbal narration, layout, and perspective. For example, in the Doll's House story arc in The Sandman, the powers of Rose Walker's "dream vortex", which threaten the stability of the fictional world of The Dreaming, are made evident to the readers by the fact that the panels of the sequence turn sideways. Later, when Rose's dreaming becomes even more powerful, the dream sequence creates a vortex on two double spreads, where the few remaining panels give the impression of having been thrown around the space of the composition.

My intention here, however, is not to expand McCloud's typology on its own terms, but to integrate the general notion of panel transition, or panel relation-rather than the gutter-to the narratological analysis of temporal order, rhythm, and frequency. Many of the shortcomings of the closure theory can be mended by Thierry Groensteen's much more comprehensive model of 'arthrology' that seeks to cover the entirety of the relations between panels in the spatial organisation of comics. ${ }^{14}$ Beyond the pure linearity of subsequent panels, the question of panel transitions is conceived here in relation to the two-directional dependence of panels in a sequence (the notion of 'iconic solidarity' between the panels). ${ }^{15}$ The main degrees of articulating panel relations in this model are the elementary, linear panel-to-panel organisation (linear or restricted arthrology) and the principle of braiding (translinear and distant or general arthrology) that concerns panel relations other than those between immediately juxtaposed images. The latter can include, for instance, interrelations between panels in different parts of the page, such as the panels at the right end of each strip on the opening page of the Tintin album The Red Sea Sharks, ${ }^{16}$ a composition that sets the two pages of a double spread in contrast or harmonious relationship (the famous central spread of Watchmen), or more distant relations between different parts of the story. The linear organisation of the panels is governed by the idea of narrative breakdown, that is, the process of dividing the narrative into images (in a strip or a larger zone of composition, such as the page), and "most often subordinated to the narrative ends", whereas braiding involves a more elaborate integration between narrative progression and the spatio-topical form of comics $(2007,22)$. Another advantage in Groensteen's multidirectional approach is that it sheds light on how breakdown and page layout interact and are mutually 
informative. Their rapport is an important formal aspect of study, given the fact that comics can vary greatly in this regard.

The all-inclusiveness of arthrology may become a problem: where should one draw the line of possibly significant relations between panels or other units of a comic narrative? However, the problem seems less serious if we consider that the salience of a given relation must always be justified, on a case-by-case basis, in text analysis. The point in identifying any 'tabular' or 'translinear' panel relations, or relations between larger segments of the narrative, is precisely to explain how the given relation is significant, for instance, in terms of temporal information, in contrast with a multitude of other relations that are not relevant to consider. ${ }^{17}$

Groensteen's capacious theoretical model of panel relations can easily accommodate those forms of temporality in comics that move beyond linear connections between single panels. These include, for instance, forms of open-ended temporal logic, or the effect of simultaneity between different storylines or two acts of telling. Think, for instance, of the dramatically different pace and tempo between words and images in the "Paper Doll" passage in Building Stories, where the old landlady tells about her memories relating to her dislike of dolls, but the reader simultaneously sees her aging in this passage from a young child into an old woman. The woman's descent down the stairs also reflects the aging process. ${ }^{18}$ Consider also the effect of the simultaneity in the panel-within-panel structure in Matti Hagelberg's imaginary biography of the Finnish president Urho Kaleva Kekkonen (1900-1986) (Figure 1.3). The arrangement in this passage allows the reader to either choose one sequence or alternate between two sequences, the small panels-within-panels storyline that depicts an interview of the cartoonist Hagelberg. The main story portrays Kekkonen, as the President, on a fishing trip to Iceland where he is swallowed by a great whale and gets to meet the treacherous Pinocchio in the whale's belly. In the interview that progresses in the corners of the main panels, Hagelberg answers the question about the sports that he would like to practise if he were elected President of Finland. The two storylines are not only distinct but also complementary; there are not only two linear sequences in the same pages but also a thematic connection through space.

Despite the many advantages of this approach, one serious narratological problem that we encounter in Groensteen's theory of spatial articulation is the argument that mere spatial arrangement of the elements of the page can somehow in itself create a sense of time. More precisely, Groensteen's notion of multiframe (multicadre) refers to the complete spatial composition in a work of comics, pertaining especially to the arrangement of panels on a page, in the sense that this arrangement interacts with narrativity (or "narrative flux", 1999, 27) and the sense of time. Inspired by the Belgian philosopher Henri Van Lier, the notion thus presupposes that spatial structure in comics has an inbuilt temporal meaning. 


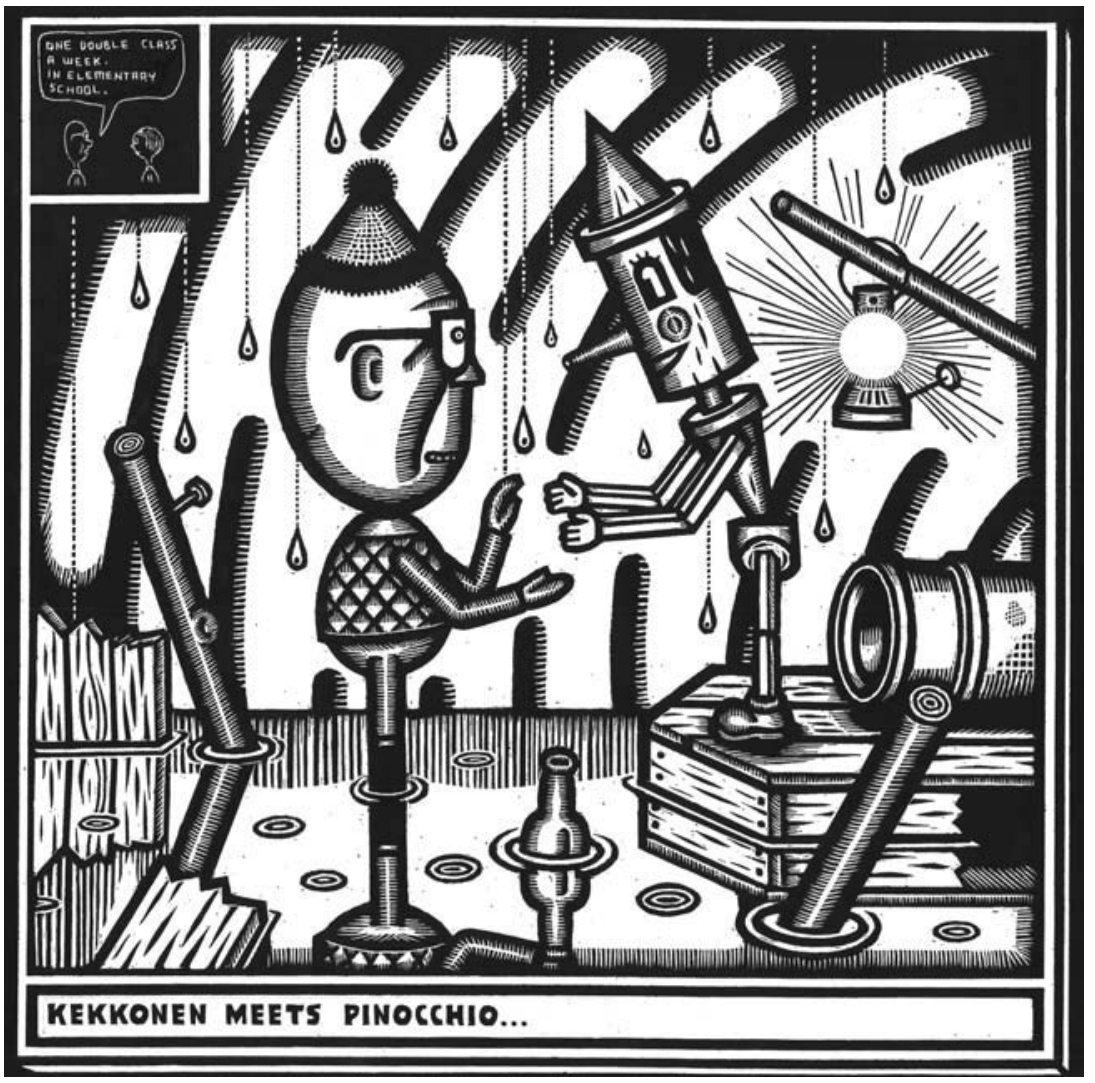

Figure 1.3 Matti Hagelberg. Kekkonen (2004) (C) Matti Hagelberg.

We can distinguish between several and not altogether compatible meanings in the notion of the multiframe. On the one hand, a multiframe refers to the total spatial arrangement of comics in the sense of a unit of reading (such as a page, a strip, or any other compositional unit). ${ }^{19}$ On the other hand, the multiframe refers to an imaginary "contentless comic" in the meaning of a pure spatial structure of a series of supporting frames, or "a comic provisionally reduced to its spatio-topical parameters" $(2007,24)$. In this second meaning, therefore, multiframe is a unit of design, for instance, the gridding of a page into a panel structure. Van Lier's perception of the multiframe is similarly flexible, involving a metaphor for an all-encompassing structural form of comics: an arrangement of juxtaposed frames that function like empty aircraft drifting in the white void of the page's space, calling forth drawn images and ceaselessly generating their mutations $(1988,5)$. The multiframe 
does not automatically produce a story or lead to a narrative $(1999,124$; $2011,16,88),{ }^{20}$ but it implies a sense of time and rhythm.

Perceived as a reading protocol, the notion of the multiframe can be useful in drawing our attention to the way in which the space of the composition can suggest connections between the panels. Similarly, studies of layout in comics have often conceived the panel arrangement in terms of rules for reading and attention. ${ }^{21}$ Perceived as the totality of the contentless frames in a comic, the notion of the multiframe may also allow us to think of the whole spatial organisation in a given work. However, beyond the very general notion of the density of the page, which relates to the number or panels in a given arrangement (Groensteen 2011,150), it is not evident how the structural matrix of empty, juxtaposed frames can represent time through space, or give rhythmic pattern to the narrative. A series of juxtaposed frames can certainly be used as an "instrument for converting space into time, into duration" (Groensteen 2013, 138), but the question remains how that kind of conversion may take place, or how spatial composition interacts with narrativity. Emphasising the space of the page as a contentless form, the notion of the multiframe cannot effectively account for the way in which space may convey a sense of time in picture stories. As our focus lies on narrative comics and narration in comics, it is necessary to develop our understanding of the narrative conventions and means of connectivity in the structure of the composition, rather than the abstract spatial form of comics.

\section{Layout and Connectivity}

Comics can, in many ways, prompt readers to find temporally and narratively meaningful relations between the panels or between a panel, a strip, and the page. In this respect, Philippe Marion has helpfully mapped out the general compositional elements and processes that assist readers in this task (1993, 220-226). These processes include:

- the means of composition and layout

- style

- the narrative content of the images

- the expectation of the reader's active processing in filling in the gaps in the story.

Many formal devices of the composition can suggest a sense of continuity between the panels. These include the use of expanding graphic elements, such as the multiplication or embedding of frames, or changing panel sizes; the contraction, reduction, or suppression of the space between the panels so that individual panels lose part of their distinction and impermeability; and the overlapping of panels to point out connections. These techniques thus create the impression of a literally 
continuous sequence or 'stream' of images in the space of the composition, while they may also undermine the integrity of the panel as a separate unit of time, space, and reading. Likewise, the page can function as a potential mega-panel that organises the relations of the individual panels in its space and demands attention to itself as a whole. One reason why Richard McGuire's nonlinear story Here (2014) is such a remarkable achievement is that the work's composition goes so deliberately against these expectations and creates its own logic of spatial connectivity. Here, the embedded frames, all identified by a year, depict one point in space, which is a corner of a room in the twentieth and the twenty-first century, thus illustrating from this fixed point the passing of time both towards the future and the past.

The spatial means of connectivity reflect and potentially encourage the two basic reading conventions that we have discussed in this chapter: the reading for the sequence and the global look across the page, or 'tabular' reading. In Benoît Peeters' much-used and debated typology of mise en page, which describes four common forms of layout in comics-the conventional, the rhetorical, the productive, and the decorative layout style - the most relevant layout types from our narrative perspective are the so-called conventional and rhetorical forms of organisation. Narrative structure dominates in the 'conventional' conception of the regular grid of panels on the page with no variation. In this case, in order to facilitate storytelling, the spatial form of the page, due to its regularity, becomes as invisible as possible. In the 'rhetorical' use of the page layout, ${ }^{22}$ in turn, the demands of narrative presentation fully dictate the dynamic spatial form in the layout. In other words, changes in the layout reflect the content of the story events. Such changes, typically, help to distinguish what is narratively salient and important in a passage or scene. For instance, in Hergé's Tintin, the panel relations, shapes, and sizes keep changing according to the evolving story, thus emphasising narrative action and the situation at hand, or in accordance with the space and time of the story.

By contrast, the so-called productive and decorative functions of layout do not have evident narrative functions. In what Peeters calls 'productive' layout style, as in Winsor McCay's Little Nemo, the changing panel sizes and shapes seem to control the story rather than vice versa. ${ }^{23}$ Here, the dimensions of the panels and the page have an expressive function, for instance, suggesting an image or an idea in their own right, or giving shape to the characters. Consequently, the evolving story appears, at least to some extent, to be an outcome of the spatial arrangement. We may thus note that the 'productive' layout refers to cases where the relation between segmentivity and narrative, or the zone of composition and the narrative, are in tension, due to the ostentatious organisation of the page that seems to dictate the narrative or give rise to narrative fragments in their own right. 
Despite its shortcomings, Peeters' approach is useful from the narratological point of view as it underscores the way in which the page layout can support temporal and narrative connections between the panels. The seemingly least narrative of Peeters' four general categories is the 'decorative' page layout, in which the page is treated, as much as possible, as an independent drawing, a design unit, or a concrete object. Yet, such a decorative panel or page can also have a narrative function. ${ }^{24}$ In his 16-album Philémon series, Fred frequently experiments with page layout to undermine the distinction between a single panel and the temporal progression of the action. One case in point are the double-spread images that Fred uses to repeat a particular panel, or a part of a panel, in the ongoing story. Typically, there are three to four such double spreads per album. These expanded panels, usually without the verbal content that they may have in the smaller versiononomatopoeia exempted-can be viewed as painterly re-framings. At the same time, the expanded panels contribute to the story by giving a more detailed description of a situation and the world of the story, while they also expand a particular moment. Moreover, these double spreads are a means of altering rhythm, creating a sense of momentary stasis in the story. The original reason for their inclusion, however, seems to have been purely pragmatic: the need to complete the required forty-two or so pages that make a comics album, thus developing the story from the pages that were first published in the French comics weekly Pilote (the series started in 1965 , the first album came out in 1972). Here again, the narrative analysis of comics confronts the question of the publication format, and the requirements of the printing technology, in shaping the narrative forms.

Beyond layout and the space of the composition, graphic style and the image content also suggest panel relations and transitions. Typically, the persistent identity of the graphic trace and style-at least to the extent that the cartoonist's style remains consistent-can imply a sense of continuity between the panels. This pertains, for instance, in Fred's Philémon series, equally to the coherence of the event and the storyworld. The change or rupture of style, in turn, can indicate a significant discontinuity in this respect. Equally, the repetition or juxtaposition of particular elements of the story, such as characters and their actions, a continuing character's perspective, features of the storyworld or situation, or the repetition of some other subject matter from panel to panel, can create narrative connections. Patterns of repetition and juxtaposition, furthermore, often rely on the expected relatedness of certain things in our world. Juxtaposed facial expressions, for instance, can indicate an emotional state and sense of involvement; doors suggest that there is an entrance into another space; falling leaves connote autumn; and so on. I will discuss the narrative functions of style and continuing characters and their actions in later chapters. 
Marion's last category of connectivity in comics is, in fact, not a particular device or strategy specific to the medium, but involves the general expectation that the sense of continuity between panels is established in the reading process (1993, 220-226). This important point goes almost without saying: the reader has an active role in negotiating and neutralising the inherent fragmentation of the narrative breakdown in comics, stringing the disparate elements together. Here we, thus, return to the issue of how to make meaningful connections between different units of the composition and the story, such as the perception of narrative meaning and coherence between the panels. The compositional means of the page layout also reflect the reader's choices in reading, for instance, by emphasising a particular panel, opposition of panels, a sequence, a sense of depth, the global look, or something else.

\section{Levels of Sequencing}

In order to study the logic of connectivity further, we can turn to literary stylistics where narrative sequencing has been conceived in a more multi-faceted and reader-oriented way. One particularly helpful point of departure in this respect is Geoffrey Leech and Mick Short's approach, in Style in Fiction (2007, 2nd edition), to narrative fiction that considers three basic principles of narrative organisation on which writers rely in determining the choice of a particular kind of sequence. These categories-chronological, psychological, and presentational sequencing-apply in a general sense, mutatis mutandis, to comics storytelling. The model allows us to consider narrative order, respectively, from the viewpoint of chronological order, the character's perspective and experience, the reader's response, as well as expectations implied in the comic, based on the author's concern for the reader's interest in the story. In actual narrative comics, all these dimensions are mutually inclusive and interactive.

The impression of chronological order follows the presumption that the passage of time in a narrative imitates chronological real time; that is, it reflects an order where an event is represented before another event simply because that event takes place before the other event. Chronological sequencing, therefore, coincides with the narratological study of order and anachrony-how the sense of chronology is created, and how the presentation of the events may digress from it.

Beyond chronological sequencing, we can distinguish two other principles of sequencing that contribute to a temporal logic between the panels. Psychological sequencing can take precedence over the chronological principle, especially in fiction. In psychological sequencing, the textual order of presenting the narrative, that is, discourse-time, "reflects the order in which impressions occur in the mind" (Leech and Short 2007, $190)$, that is, as they occur in a particular character's or narrator's mind 
and perception. This involves, perhaps most importantly, the portrayal of events, and their chronologies and causal connections, viewed through a sentient agent or an experiencing consciousness. One seminal technique in this regard is the use of fictional point of view (or internal focalisation) where information about the events and situations of a story is limited to a particular character's mind and perception. Another common method is first-person verbal narration that focusses on the narrator's thoughts and emotions, or the use of subjective viewpoints.

In comics, as in literature and cinema, psychological sequencing contributes to the order in the arrangement of panels. Sometimes, especially in stories that focus on a particular character's experience or psyche, this principle may become particularly prominent. ${ }^{25}$ Such passages can, for instance, be organised along psychological continuities that the reader establishes between panels and their groupings and associates with a certain character. For instance, in Bastien Vivès's A Taste of Chlorine, various scenes at the swimming pool are subjectified through the protagonist's perspective in the images that show him looking at something or show his field of vision behind his back. By contrast, the various centralised images - masks or kinds of paper dolls - in the double spreads of Chris Ware's Building Stories, especially ones including images of the main characters' faces with closed eyes, create a psychological frame or a kind of embedding for the viewing of the surrounding panels by association. In Jeffrey Brown's autobiographical graphic narrative Clumsy (2002), in turn, the diary-like perspective and style of drawing suggests that the non-chronological episodic structure of this work, which depicts a one-year long-distance relationship through a series of significant situations and scenes, reflects the order in which the impressions may have occurred in the author's mind. The emphasis lies on the personal experience of time, not chronology.

In addition, what is equally significant in terms of psychological sequencing are the potential, but unrealised, alternatives in the evolving eventsthings that could happen but are not realised. A character may anticipate or hope for an event that does not take place, goals may not be attained, a retrospection of past events may be unreliable, and the narrative may foreshadow events that do not take place. Such hypothetical, unrealised, temporal scenarios can have a significant role in the story and its reading. ${ }^{26}$ Thus, it may be useful, in order to perceive temporality in narrative comics more fully, to think beyond the question of anachronies and mere sequencing, to the relation between the chronology of the events and the network of unrealised possibilities that evolves as the narrative progresses.

Finally, the principle of presentational sequencing considers the logic of narrative sequence from the viewpoint of intelligibility and narrative tension. We can conceive this principle also as the cartoonist's or the storyteller's concern for the audience's interest in the projected sequence. Thus, choices in the sequential organisation of narrative comics are 
dictated by the potential of the given organisation to create kinds of audience responses that encourage the reader to keep reading. Leech and Short formulate the principle as a question: "What is the appropriate order in which the reader should learn the elements of the fiction?" $(2007,143) .^{27}$ Thus, presentational sequencing reflects the cartoonist's choices that are dictated by the necessity to gradually build a sense of a world, an event, or a character and, moreover, to do this in a way that maintains the audience's interest in the evolving story. The building of narrative tension also has an artistic dimension, i.e. the art of holding back information so that it heightens interest in and curiosity about the story, or creates effects of surprise and suspense. This is, then, another motivation for the divergence of story-time and discourse-time: their distance may increase the reader's interest in the story. Presentational sequencing is operative at several levels simultaneously, including the order of the events, their experiential order, and their psychological significance for the characters, as well as knowledge about the characters-all perceived through the author's and the reader's shared interest in the evolving story.

Another rationale for distinguishing presentational sequencing from chronological and psychological levels of sequence is that the structures of temporal order in narratives cannot be totally cut off from the issue of knowledge about the fictional world. The manipulation of the chronological order has various effects on our knowledge about the story world, characterisation, the salience of a particular experience or situation, and so on. One basic rule of intelligibility is that things that require less background information will come first in the story, that is, that the story develops "from elements which presuppose the least prior knowledge to those which presuppose the most" $(2007,143)$. If this rule is undermined, as happens in Building Stories, the transgression should serve a particular purpose, since discourse-time is thus foregrounded. The comic may start in medias res, or important information concerning the story world is postponed, and this increases narrative tension in the tale.

In actual narrative comics, the three types of sequencing coincide and create combined effects. The manipulation of chronology can, for instance, bring the character's mental state into better view, or a scene shift may reflect transformations at all of these levels at once. Finally, it needs to be stressed that forms of nonlinear temporal order, ambivalent time frames and unrealistic time are also possible in comics, including types of circular or contradictory time. ${ }^{28}$ The creation of such effects may require the undermining of all these levels of sequencing and, thus, the applicability of the narratological model can be noticeably limited.

\section{Duration and Rhythm}

In literary narratives, as in comics, textual features or cues cannot measure the sense of duration, speed, and rhythmic alteration to the 
same extent as we can observe anachronies in the order of the narrated events. This is because impressions of duration and rhythm are dependent on the reader's subjective sense of time in reading. One reader may spend a lot of time with some scene, while others a lot less. Nevertheless, we may be able to analyse duration and rhythm in comics in a relative sense by comparing the ratio between the length and complexity of the representation of an event-in terms of panels, strips, or pagesand the time span that is covered by that event or situation in the world of the story. The approximate duration may then be estimated on the basis of the number of panels (per page, tier, or scene), the number of pages (per situation, event, or scene), the amount of textual and visual detail in the images, and the complexity of panel relations and the layout in comparison with the length of time passed in the world of the story, provided that there is sufficient indication about the passage of time in the story. 29

By 'duration' is thus meant the relationship between the time of the events in the story, i.e. their duration in the time of the story, and the space given to their representation, and the time of reading that this space implies. Narrative rhythm or tempo, in turn, can be specified as the alteration of this ratio, for instance, through changing patterns (cadence) or speed. Such rhythmic effects are generally more observable within larger narrative units, such as a scene or between two scenes that allows us to make observations about significant changes in this regard. In Ware's Building Stories, for instance, narrative rhythm is sped up considerably in a 'staircase' sequence of about twenty panels that shows the protagonist's landlady age from a young girl to an old woman about eighty.

In the Philémon series, Fred regularly investigates narrative rhythm by introducing two simultaneous temporalities and spaces in the zone of composition so that the panel division on the page reflects, at the same time, one divided space at one point in time and an event that evolves in the same space (Figure 1.4). Other techniques for slowing down and at once complicating the effect of rhythm include the above-mentioned double-spread wordless images or page-size panels, which draw attention both to the sequence and the composition as a whole. The Philémon series also frequently features panels that have an ambivalent status as two-dimensional visual fields, such as panels that flip over or become objects. These instances of 'metaleptic' 30 panel constitute another breach of the level of reality in the story: the characters relate to the formal elements of comics as objects or as spaces that they can explore at will. In other words, the spaces of the layout transform into parts of the fictional world, thus collapsing the border between the space of the composition and the narrative space.

Duration and rhythm in narrative comics are relational terms also in the sense that they correlate the relationship between the time of the 


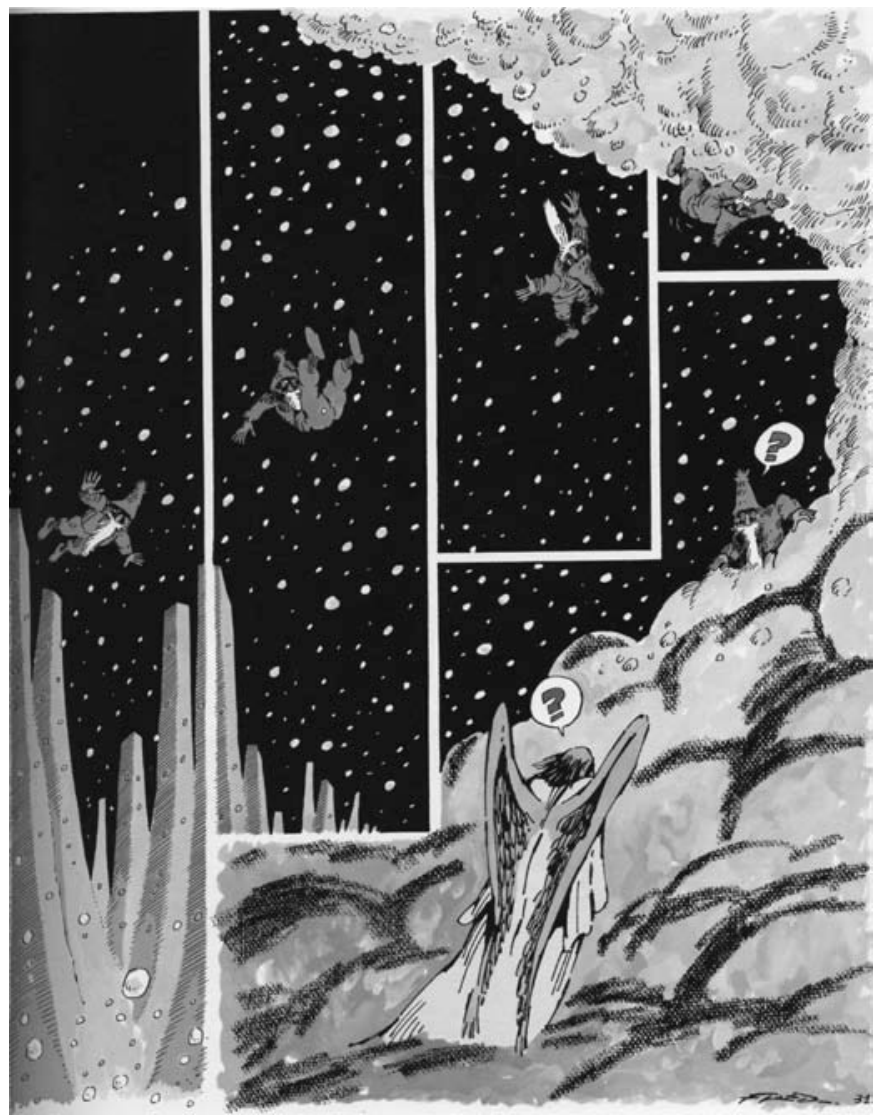

Figure 1.4 Fred. Philémon. La mémémoire (1977) (C) Philémon-tome 11, La mémémoire DARGAUD by Fred. All rights reserved www. dargaud.com.

events in the story and the space given to their representation in some narrative unit, such as a scene, with another unit or the narrative as a whole. In literary narratives, films, and comics alike, variations of tempo involve a number of conventional patterns that distinguish, for instance, a scene from other scenes, or between different modes of narration (dialogue, thought report, representation of action, and so on).

The manipulation of rhythm is perhaps even more frequent than anachronies in narratives, regardless of media. In fact, Gérard Genette has argued in relation to literary narratives that "it is hard to imagine the existence of a narrative that would admit of no variation in speed-and even this banal observation is somewhat important: a narrative can do without anachronies, but not without anisochronies, or, if 
one prefers (as one probably does), effects of rhythm" (1980, 88, italics original). Reflecting on the conventions in the history of the modern novel, Genette then goes on to typify four canonical forms of novelistic tempo, or what he calls narrative movements. These include the two extremes of ellipsis (maximal speed) and descriptive pause (maximally slow rhythm), and their two intermediaries: a scene, most typically in the form of a conversational scene (dialogue), and which "realizes conventionally the equality of time between narrative and story", and a summary. Summary, as Genette specifies, typically has a variable and greatly flexible tempo, which "covers the entire range included between scene and ellipsis” (1980, 94).

Genette schematises the conventional rhythmic variations, or canonical forms of novelistic tempo, with the following formulas, where $S T$ designates story-time and NT discourse-time (or narrative time), the sign $\infty$ meaning either infinitely greater $(\infty>)$ or infinitely less time $(<\infty)$. 0 indicates elision, that is, either that a section of discourse, such as a descriptive pause, corresponds to no duration in the story, or some part of story-time is absent from the narrative (ellipsis) ${ }^{31}$ :

pause: $N T=n, S T=0$. Thus: $N T \infty>S T$

scene: $N T=S T$

summary: $N T<S T$

ellipsis: $N T=0, S T=n$. Thus, $N T<\infty S T$

What is left out from these formulas is the fifth logical option, i.e. that the effect of rhythm in discourse-time could be slower than in story-time (NT > ST). Genette admits that this rhythmic formula may be possible, but only in experimental narration- "the reading of which often seems to take longer, much longer, than the diegetic time that such scenes are supposed to be covering" $(1980,95)$ - or in slow-motion techniques in film. Later, other narratologists have problematised this exclusion. Beyond literary experiments with repetition, where the same event or the statement of that event recurs several times, Seymour Chatman argues that the verbal rendition of a character's mental events is bound to be much slower than what has transpired in the character's mind $(1978,73)$. Chatman thus adds the fifth formula, stretch $(1978,72-73)$, to the basic variations. For Christine Brooke-Rose, the missing formula of NT > ST, or what she calls the 'slowed down scene', has an even broader scope than this. She argues that any narrator's commentary on "gestures or sighs or sinking hearts", or "thoughts, memories, emotions and observations" in a dialogue scene, expand the scene to "a much 'slowed down scene"” (1981, 315). Therefore, Brooke-Rose argues, NT = ST can be reserved for pure dialogue only $(1981,315)$. Consequently, there should be plenty of room to consider the rhythmic formula of NT > ST in fiction. 


\section{4}

Time in Comics

All five basic types of narrative rhythm can be found in comics storytelling. In fact, in visual narratives, such as films and comics, the rhythmic formula of stretch seems to be one of the basic rhythmic variations. In film narratives, a stretch may be realised, beyond slow motion, by a number of techniques, such as overlapping (or repetitious) editing, types of camera movement- the camera moves slowly around close-up details of an image or a scene without action, for instance-and an extended shot of a static subject. In comics, cartoonists have various graphic and spatial techniques at their disposal to slow down discourse-time considerably. This can be done, for instance, with a descriptive passage that shows various aspects of the same object or scene in the same instant, a page layout that breaks down the sense of temporal progress by means of multidirectional or ambivalent panel relations, or a scene where hardly anything changes from one panel to the next. One striking example of a stretch is a page from Scott McCloud's The Sculptor (2015), where the protagonist, the sculptor David Smith, is lying on his bed with his lover, Meg, seen from above, and becomes excruciatingly aware of the passing of time. Here, a close-up image of David's face is replaced, in a sequence of panels, by four images of a clock on the wall in a kind of zoomed-in effect. The hands of this clock are not moving, but we 'hear' it ticking six times-as shown in the gradually growing and ever bolder letters of an onomatopoeic 'tic' that accompanies the panels, depicting the clock and again the protagonist's distressed face. This suggests that the length of the scene is only a few seconds. In this time, the protagonist, becoming aware of the clock's ticking, closes and opens his eyes. The last panel on this page, a bleed image in the corner of the page, shows David's horrified face with the last 'tic' superimposed on his mouth.

To summarise, the techniques for diversifying narrative rhythm involve:

- means of layout:

- changing panel size, shape, or colour

- rhythmic function of the panel frames ${ }^{32}$

- changing layout style

- creating relations between the panels and the page layout that undermine the expectation of temporal progress through linear sequence (many panels form one image, page as a geometrical shape, stacked panels, etc.)

- rhythm-activating and reinforcing relations between speech and thought balloons and captions (or words in the images) in and across the panels

- means of drawing and style:

- change of style

- collapsing the distinction between the spatial composition and the narrative space and time

- increasing density of visual information 
- means of verbal narration:

- changing amount of verbal narration and information

- narrative summary that speeds up narrative time

- distancing verbal narration from visual information

- size of the page and the form of the book.

All narrative comics share the basic expectation that their space can be perceived in terms of duration. However, depending on the narrative context, the panel shapes and sizes, sequences, and layout techniques, or other formal aspects of comics, can suggest a great variety of effects of temporal duration. To use Thierry Groensteen's formulation of this point, there is no automatic correspondence or direct proportionality between the shape or size of the panel and the duration of the action presented in the image $(1999,56)$. Instead of conveying some standard temporal effect, such as an increase or decrease in speed, the relation between panel sizes, sequences, and duration is only suggestive of some temporally organised meaning, or a condition of such meaning. A series of multiplying panels can create an impression of speed, as happens, for instance, in the flashback passage in Prosopopus, but the multiplication of panels may equally well indicate the impact of repetition, or give an effect of slow motion. Therefore, the nature of the relation between spatial form and temporal effect needs to be estimated on a case-by-case basis. Ultimately, the sense of time in each picture panel, sequence, or other narrative unit depends on the context, i.e. the local means and aims of narrative breakdown, the relations between the panels, and the reader's active cognitive mapping of these relations.

\section{Duration in a Single Panel}

It is another commonplace in comics theory to argue that an individual panel represents a well-chosen moment in narrative action. ${ }^{33}$ A sequence of panels, then, can be said to be composed of a series of such discrete moments. ${ }^{34}$ However, the 'panel is a moment' thesis is problematic for at least three reasons. First, not all panels are so much moments as they may be perceptions, perspectives, thoughts, ideas, or representative scenes of action. Second, single panels in comics frequently represent duration, a span of time, instead of a moment. Third, if all moments in comics are inscribed in a sequence (or other form of grouping), this opens the question of the interdependence between the supposed moments and, subsequently, about their lack of distinction as a moment.

Usually, when the 'panel is a moment' thesis is made, it is accompanied by one exception that is too obvious to dismiss, which is that speech necessarily introduces a sense of duration in a single panel. In actuality, however, there are many other exceptions: descriptive panels and passages; sequences that follow a character moving or doing something; 
images that describe a character's emotion, attitude, or thought; or the first-person narrator's introspective passages that do not portray charged moments. Verbal narration in captions can imply a sense of duration for the visual contents of the image or frame the image in terms of such duration. Captions can specify whether what is seen in the panel has a certain duration, or they may include insertions, commentary, and description that clearly lengthen the sense of duration in a scene. Importantly also, visual information, the organisation of the field of the image, and specific techniques and conventions of drawing can introduce duration in a single panel. The latter include conventional signs and symbolic tricks, such as motion lines, and the use of large panels to create a different sense of duration or, possibly, an (infinitely) extended moment. A panel can also contain so much visual information and complexity, or so many distinct instants of action, that this can be comparable to reading a sequence of panels. Furthermore, the demands of the 'iconic solidarity' of images in a sequence dictate that the unit of time depicted in a panel is rarely a single instant, but opens to the previous and subsequent panels, or the surrounding ones, and the temporal frame that they develop.

In her brief application of Genette's and Brooke-Rose's model of narrative rhythm to single panels, Julia Round has made a thought-provoking point about duration in individual panels by claiming that comics can enhance the distinction between a scene and a stretch $(\mathrm{NT}=\mathrm{ST}$ and NT > ST) in a single panel. This can happen in two essential ways: by way of the amount of visual detail in the panel, involving, for instance, the characters' posture, bodies, and signs of emotions, or through the relationship between the image content and verbal narration. More precisely, Round points out that panel images, when they depict scenes where story-time and discourse-time seem identical $(\mathrm{NT}=\mathrm{ST})$ and also when they are without any verbal narration ('pure description'), can include visual and emotive elements that increase narrative time. When panels that depict a scene feature verbal narration in captions, they must then be defined as NT > ST. Thus, discourse-time becomes clearly longer than story-time $(2007,322)$.

There exist various more elaborate visual conventions and layout techniques by which cartoonists can introduce a sense of duration in a single image, suggest an ambiguous relationship between a moment and duration, or undermine the idea of the panel as a distinct unit of time. ${ }^{35}$ These techniques comprise, for instance, the use of superimposed images or parts of the image that show several positions of a person or an object simultaneously, or the distribution of different phases of the same movement among several similar characters in the same image. A panel may also show objects from different temporal strata, superimposed or collaged in one, for instance, as a reflection of a character's or a narrator's memory. The repetition of the same figure in the space of one image is another option. Such multiphase images may, for instance, depict an 
evolving action, different phases of a character's movement, or a whole event. ${ }^{36}$ Still another example of multiple perceptions in a single panel is the use of split panels, including panels such as those in the Philemon series, where a larger segment of the story, perhaps a whole page, can be seen both as a sequence of panels and one image that stretches over the whole page. This technique can to some extent be associated, as Scott McCloud suggests, with the art history term polyptych, which refers to a painting divided into sections. With this technique, cartoonists can deliberately ambiguate the distinction between a moment and duration, or a single image and a sequence. They may also, vice versa, turn a potential sequence into a single panel.

One variation of the complex polyptych panel are 'splash' pages and the use of large panels as spreads. Typically, a splash panel evokes movement or a series of actions in static form. Thus, cartoonists have, to borrow Charles Hatfield's phrasing, expressed "extended spans of time in synoptic fashion” $(2005,54)$. Jack Kirby's crowded spreads typically capture explosive moments of action. By contrast, Fred's page-size panels or double spreads slow down the narrative rhythm, depicting, thus, an extended moment within the action or, perhaps rather, action as a kind of painting that suggests a longer duration. Similarly, the use of 'bleed' images, where the image runs off the page, and which are especially common in manga, can evoke a variety of effects of time, including the sense of timeless or boundless space, or a much-extended moment.

Not all cartoonists employ these techniques, but they are illustrative of the inherently dynamic, flexible, and heterogeneous nature of the panel as an imagined unit of time and duration. The crucial point to make here is that single panels vary greatly in the way in which they evoke a sense of duration. At one extreme, panel images suggest no change in the temporal frame of the depicted event or situation. Other panels, in turn, depict minute changes from panel to panel, such as single instants of movement or action. Some panel images may encapsulate dramatically, narratively, and emotionally charged phases of an event or situation that point to some before and after of the depicted scene, while other panels depict long duration or many simultaneities. Ultimately, however, all effects of duration in a single-panel image rely on the reader's processing of the given panel's relation to the other panels around it and their understanding of the evolving narrative action, event, or situation.

\section{The Problem of the Key Frame}

One regular effect of rhythmic variation is narrative saliency. By this I mean that changes in rhythm help to make certain local features of the narrative, such as a single panel, a strip, a passage, or a scene, contrastively salient in relation to other units around it. The point has been made by several narratologists, including David Herman, who argues that 
"Functionally speaking, longer or shorter duration can cue readers to focus on some narrative details as more salient than others" $(2002,215)$. A change of rhythm may, therefore, also highlight what narratology calls the cardinal function of an event, that is, an action or event that is logically essential to the narrative action as a whole. ${ }^{37}$ Typically, a cardinal function determines a causal sequence, such as resolving an instability, uncertainty, or tension in the narrative which have been of interest to the reader, or opening up new questions, instabilities, and uncertainties. In addition, rhythmic changes may indicate narrative salience in relation to transitional moments and passages. The acceleration or slowing down of narrative rhythm can then raise expectations about a significant event that is approaching. ${ }^{38}$

The interaction between rhythmic variation and narrative salience is a highly common feature in comics. Let us think of their relation in the French-Canadian cartoonist Guy Delisle's documentary travel story, Pyongyang: A Journey in North Korea (2003), which describes the author's experiences during a 2-month period of overseeing the production of an animated work at a film studio in the North Korean capital of Pyongyang. What is of particular interest to us here is how the notion of key frame, used in filmmaking to refer to drawings of important frames of a sequence, is treated as part of this parody, and how the notion of a key frame might be conceived in relation to narrative rhythm.

A passage in Pyongyang that highlights for us the relation between narrative rhythm and salience includes a visual citation taken from Hugo Pratt's Corto Maltese story "L’ange à la fenêtre d'orient" (1971), originally included in a collection entitled Les Celtiques (Celtic Tales, 1980). The image shows the hero, Corto, about to shoot a revolver into the air. This citation is, in fact, a reframing: Pratt's original panel is reframed, and within this new frame, there are two pieces of additional verbal information, namely the words "Corto Maltese" above the image and, below it, the reference to comics as the ninth form of art ("Corto Maltese-Monument of the $9^{\text {th }}$ Art"), a notion that is widely used in Francophone comics culture and scholarship. Thus, the same panel is a unit within the sequence of Delisle's story, a visual citation that invites us to think of the relation between this travel book and Pratt's original story, and particularly in relation to the cartoonists' task of turning Pratt's album into an animation film. Moreover, the citation functions as a parodic homage, and a kind of monument to the comic book as an art form.

The narrative context of the embedding draws attention to the way in which comics heroes, such as Corto Maltese, are adapted in animation film. The citation is placed within a scene containing conversation, set in a Pyongyang café, where one of Delisle's colleagues explains that he is currently working on a Corto adaptation. In the subsequent panels, the author-narrator explains that to minimise the production expenses 
of the production, animators in Paris drew only the key frames, whereas assistants in North Korea did the in-medial frames. The idea of a key frame is further illustrated on the bottom of the page by a series of eight small images of a simple anthropomorphic figure making hand gestures, and the accompanying quiz-style question: "Which of these are key frames?" The answer to the question-the first, the middle, and the last position-is given on the bottom of the page and can be seen if the page is turned around (Figure 1.5). At the end of the conversation, Delisle's autobiographical character comments disparagingly on the Corto project, saying "Great, that way kids don't have to bother reading books. They'll just think everything started on the TV, like Tintin".

What happens is that the double-framing, the caption, and the intertextual gesture (recalling to the reader's mind the original Corto Maltese story), slow down the narrative rhythm. The effect of arresting narrative flow is accentuated by the fact that the original Pratt panel depicts a central moment in the action. To illustrate the point further we can consider the original panel sequence from which the given picture is borrowed. In this passage, we see the adventuress Venexiana Stevenson escaping in an airplane from the hands of Venetian guards and of Corto himself. The manipulation of a time lapse between the panels, and the changes in perspective from one panel to another, reveal Corto's spurof-the-moment decision not to shoot at the plane at which he is aiming. However, this we only learn by reading the whole sequence. What first appears like a moment of transition turns out to be a decisive moment of non-action that is essential to the scene as a whole. It is a key situation without which the narrative would not be the same. Recontextualised in Delisle's travel story, however, the Corto panel loses its cardinal function

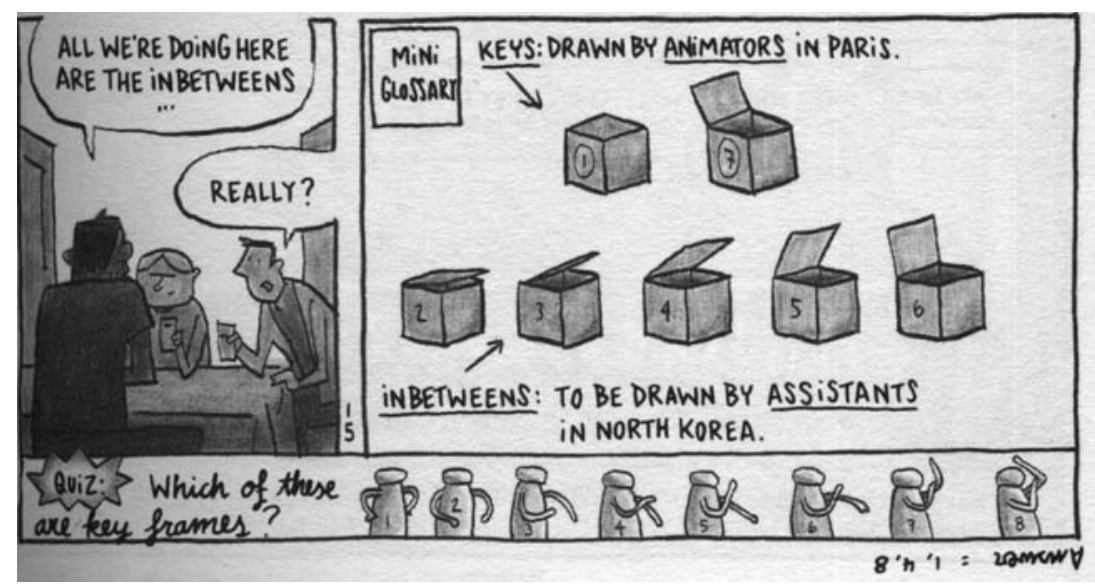

Figure 1.5 Guy Delisle. Pyongyang: A Journey in North Korea (C) 2006 L'Association/Guy Delisle; courtesy of Drawn \& Quarterly. 
or, perhaps rather, is expanded with additional meanings. The doubly framed panel stands for a key frame; it is a symbol of key narrative action in comics, but it also shows how the notion of the key frame is not similarly relevant in comics as it is in animation film.

In comics storytelling, to show a particular image and to frame it is, at the same time, to give emphasis to what is shown and call attention to what is drawn. ${ }^{39}$ This is one reason why the notion of a key frame becomes problematic. Every panel and image in comics already represents, in a sense, a frame that shows and tells the reader narratively salient information (even if there is no actual frame). The panels also do not refer to a pre-existing extratextual 'flow' of visual information and continuing event, as in acted films, but create a sense of that flow by referring to it. This is at least the default expectation.

The question of salience in a single panel is also relative to two functions that single panels can have in narrative comics: their painterly function or their narrative function. The distinction, first developed by Pierre Fresnault-Deruelle and Benoît Peeters, has been given weight by Philippe Marion, who has argued that the comics panel can be seen as an expressive fragment traversed by two contradictory dimensions: the story and the picture $(1993,212)$. The picture function encourages the reader to arrest his or her attention on a single image and isolate it from the narrative continuum, while the narrative function prompts the reader to glide over the image in order to grasp the sense of the continuum. ${ }^{40}$ The picture function of the panel, therefore, urges the viewer to spend more time with the panel. A given panel can, for instance due to the amount of visual detail that it contains, the complexity of its contents, the skill of the artwork, or the sheer size of the image, call attention to itself as a single image, or a work of visual art that encourages its appreciation as a distinct unit of design. An extreme case of the picture function would be a maximally self-sufficient image, or what could be called a 'memorable panel', which may risk jamming narrative responses to the image. Roy Lichtenstein's famous enlarged and altered comic strip panels from the early 1960s turned single panels, perfectly cut out from their narrative context, into a work of art. Thus, the painterly panel draws attention to itself by making the narrative time of the panel as long as possible, giving the impression, as much as that is possible, that a single panel can stand on its own.

\section{Frequency}

Frequency is the third and last determinant in the relationship between story-time and discourse-time in Genette's model of narrative temporal organisation. Variations in this regard are based, on the one hand, on how often a particular event of the story is recounted and, on the other hand, how often a narrative statement concerning an event is repeated in the story. Thus, Genette distinguishes between four basic types of 
relations of frequency that he calls 'singulative narratives' (two forms), repeating narratives, and iterative narratives:

a singulative a: a narrative tells once what happened once;

b singulative b: a narrative tells $n$ times what happened $n$ times;

c repeating: a narrative tells $n$ times what happened once;

d iterative: a narrative tells once (at one time) what happened $n$ times.

The first form of singulative (a) in this scheme involves a 'natural' correspondence between the narrated events (of the story) and the narrative statements (of the text). Thereby, something unique and singular happens once and is told once $(1980,114)$. By contrast, the more general formula of singulative (b) is a pattern of repetition where several similar events are recounted several times (as many times as they occur). In this case, repetitions of the narrative correspond to the repetitions of the story $(1980,115)$ so that Donald Duck is humiliated several times in the same story, Corto Maltese is repeatedly shown smoking, or Wonder Woman confronts several villains in a row. Like the first type, singulative (b) also follows the natural frequency of the events, or the baseline mode of assumption, where each event is perceived as unique and only occurs once. By contrast, repeating and iterative narratives (c and d) comprise at once more obvious and more elaborate cases of repetition and manipulation of the relationship between an event and its recounting.

In comics, all cases of singulative, repetitive, and iterative, and their various combinations, are equally possible. Yet, what is specifically challenging to the analysis of narrative frequency in this medium is that we need to take into consideration repetition at various levels of representation: the images, the layout, visual style, the words, and their interaction. For instance, on one wordless page in Bastien Vivès's graphic novel A Taste of Chlorine, the sense of repetition in action is created and reinforced by at the same time the means of perspective, visual showing, and the means of layout (Figure 1.6). The nine panels of the page, organised in three tiers on a regular grid pattern, show the swimming protagonist turning at the same end of the pool three times. The perspective of the panels always remains the same, thus emphasising the effect of repetition, i.e. the swimmer's repeated movements in the corner of the pool. Visual details of his movement, strength, and speed further suggest that the swimmer's technique keeps improving and, further, that time passes between the three depicted lapses. The layout has an important function in suggesting the sense of repetition here since similar small panels in a regular grid are not used elsewhere in the story. In terms of Genette's model, the case represents a blend of singulative (b), where the narrative tells $n$ times what happened $n$ times, and an iterative, where the showing implies that the action has in fact happened many more times than what is shown. 


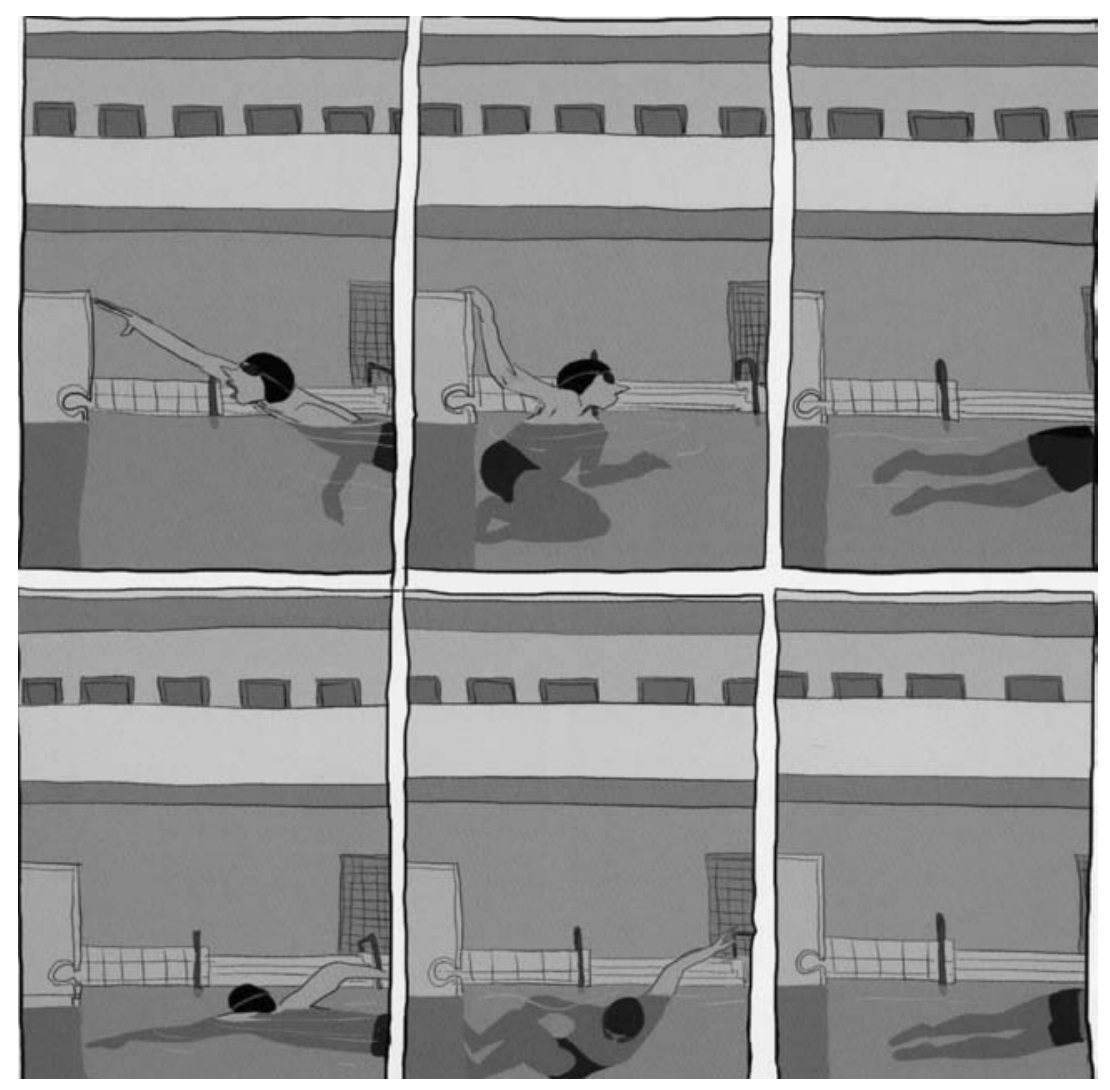

Figure 1.6 Extrait de l'ouvrage A Taste of Chlorine (2011). Bastien Vivès (C) Casterman. Avec l'aimable autorisation des auteurs et des Editions Casterman.

A caption or dialogue can give the accompanying image an iterative meaning, indicating that what is seen in the panel has, in fact, happened many times. In turn, the repetition of a whole panel content, that is, a series of two or more panels where nothing changes, may generate various temporal, spatial, and dramatic effects, such as the impression of dramatically slowed down duration, as happens in some of Régis Franc's works, or in the Oupabo experiments with 'iconic reiteration'. ${ }^{41}$ Similarly, the use of superimposed or partly overlapping images can suggest the iteration of the recounted event or the act of recounting. Different effects result again from repeated scenes if they are closely associated with a particular character's perception. Thus, repetition in the image content becomes a product or reflection of the character's mind. 
What is especially useful in this model for the analysis of narrative comics is that it allows us to think of the various ways in which repetition (cases b-d), can be used to create particular effects in the course of the story. Repetition may suggest, for instance, a range of temporal impressions, such as a sense of circular time, underline the interrelatedness between different events, situations, or experiences, or undermine the idea of an authentic single event. Repetition is also a means of emphasis and narrative salience: it may help to signal the importance of something, be that an idea, emotion, phrase, word, experience, behaviour, memory, action, or an event. The repetition of the same panel may thus amplify what is shown-making it more significant from the character's or the reader's perspective-or turn the image into a mental one, such as a memory image. Furthermore, the recounting of the same event may provide narrative action with an informative frame or background, or reveal what is usual (since repeated) in a character's life, for instance. Repetition is also another means by which storytellers can create particular effects of rhythm.

Think, for instance, of the structures of repetition in a passage called "A Feeling" in Building Stories when the main character is shown lying in her bed in various positions and at different times of her life, but apparently in the same room, in the space of a page. She is unable to sleep. The panels of this scene feature the protagonist with two different cats, a man, a teddy bear, but mostly alone, with different hairdo, body shape, younger and older face, pregnant and not pregnant, and different clothes, bed, and furniture. One event thus opens onto a pattern of repetition of similar moments and situation, some panels describing the woman at the present moment in the story, while others illustrate her memories of the same place and situation. At the same time, repetition in Building Stories creates pronouncedly polychronic effects, in the sense that David Herman defines polychronic structure in narratives (2002, 212-3), that is, having a fuzzy or indeterminate ordering of events. ${ }^{42}$ Such polychrony is related to the inexactness in a particular panel's temporal ordering, or their partial and multiple ordering in a repeated structure. For instance, repetition suggests multiple temporal orders when the protagonist is shown in three different phases in the process of going to bed in one panel (in a passage entitled "Repetition") or in four different positions (and times) in her old room at her mother's home.

It must be noted, however, that not all effects of repetition in comics have only a temporal nature. Beyond the manipulation of the distinction between story-time and discourse-time, visual repetition is a crucial element in terms of narrative coherence, continuity, and connectivity. Typically, this involves the repetition of characters, scenes, and situations, or some other image content, such as background, so as to move the story forward and give a sense of a world. 


\section{Conclusion: Time and the Reading Conventions}

By way of conclusion, we should identify those features in comics and their reading that pose the most important and interesting challenges to the narratological analysis of time in this medium. Perhaps the most obvious feature in this regard is the discontinuous spatial form of comics. A comprehensive take on temporality in comics needs to consider the relationship between a single panel, the panel sequence, and the page layout (or other forms of composition), and how comics exploit the ratio between all these compositional units to convey a sense of time. Another key feature in this regard, the possibility of seeing the whole strip, page, or a double spread at once, raises the question of the interplay between sequential and non-sequential reading. A global look at the whole page has various potential, but not always unambiguous, consequences for the experience of time in comics. The possibility of looking at the strip or the page as a whole, as a composition and a design unit, may contradict, to some extent at least, the linear following of the story. This is not entirely different from readers of literary fiction skipping to the end of the page, passage, or the whole book to know what will happen. Yet, differently from the sequential order of words on a page, gazing at the space of the story can function as a form of anticipation in itself, giving the reader some notion, especially in what may be seen in the images, of what is expected to happen.

Still another challenge that we have to come to terms with in applying the story-time and discourse-time distinction to comics is the multimodal form of expression and communication in comics. This involves the integration of words and images, and their combined forms, in sequence and into a whole; the binding of spatial, visual, and linguistic information to make sense of the gaps of the story; and the translation of certain conventional signs, symbols, or visuals in comics, such as caricature, gestural language, or kinds of lettering, as markers of emotion and thought. The issue of dual input of words and graphics, and their combined visual-textual forms, is crucial for understanding the representation of time in most comics.

Thus, to summarise, the conventions of reading comics that pertain to the representation of time include the following:

- The perception of meaningful relations between panels, in two directions before and after a panel, in a sequence, and possibly also across the space of the composition.

- The processing of the relationship between panels and layout, in particular when layout style invites a more global look.

- The processing of mutually reinforcing indications of layout, style, verbal narration, and information, and the narrative content of the images. 
- The processing of the effects of bimodal reading and the dual input of words and graphics: one modality can help to fill the gaps of temporal information created by the other modality, but words and images also expand each other's meanings, set in a meaningful contrast, or words can slow down the narration.

- The attention to synchronic reading of, or global look at, the whole of the composition, typically the page or the double spread in the longer formats. Readers can always choose to look at the composition as a whole. At the same time, comics vary greatly in their capacity to invite a global look.

- Expectations concerning the time of reading related to the genre and the publication format.

The techniques of sequencing and page layout precondition these conventions. Therefore, on the one hand, comics evoke a sense of time through a sequentiality that pertains to the relationships between the panels and their narrative breakdown. On the other hand, comics can imply forms of temporality in the overall spatial arrangement of panels on the page, encouraging a synchronic look at the whole of the composition. Both aspects of temporality are intricately intertwined in the composition, but they may sometimes exert independent effects of time. That is a challenge to the narratological approach, but we can also respond to it. We can separate these compositional arrangements on a heuristic basis, keeping in mind that the various forms of the layout, and in particular the panels and their frames, are potentially relevant both in terms of space and time, and as forms of organisation (or design) and attention. The varying possibilities of introducing a sense of duration in a single panel point to the fact that the tension between linear and synchronic or tabular reading can take place within a single panel as well; also, contrasts between two or more panels of varying size, shape, or visual complexity can create that effect.

At the same time, it must be granted that the idea of global look or tabular reading may not be that relevant for all kinds of comics, or its not similarly relevant for all forms of layout, especially in respect to traditional newspaper strips, comic strip booklets, or comics with a simple grid-like organisation. In these cases, reading is not simply linear either since regression, i.e. returning to the previous panel, seems always an important aspect of reading, or it may also happen, for instance, in Ware's comic strip booklets in Building Stories, that two parallel strips create the effect of two simultaneous sequences. Yet, such comics do not invite, let alone demand, a global look. In these cases, readers can certainly always choose to look at the whole strip or page at first, but a distinction can be made between such a glance and a global look that is anticipated and invited by the layout and, in a sense, built into it. 
We should, however, resist a too easy opposition between the linear and the tabular (or synchronic) reading. ${ }^{43}$ One good reason for not reducing their relation to a simple opposition is that the notion of tabular reading perhaps covers too much ground. In its current use in comics theory, the notion has a kind of all-encompassing quality with regard to various forms of non-sequential composition and reading. One ambiguity in the notion is that, on the one hand, it refers to specific techniques of nonlinear and/or non-sequential panel arrangement on the page while, on the other hand, it may also indicate a manner of reading comics in general, including also those comics that strictly follow a linear arrangement of panels in a sequence. It needs to be stressed that a 'tabular' reading is not a technique of composition in itself, but a convention of reading comics that the compositional choices in the work, such as a nonlinear grouping of panels, may encourage. Still another ambiguity in this notion is that it can refer to the strip, the page, or the double spread, or other zone of composition, as a unit of reading and a unit of design (involving a picture, a painting, or a surface) at the same time. This may not be totally avoided since forms of design, composition, and publication reflect and shape conventions of reading in comics, but narrative theory and analysis should at least be aware of that duality.

The global look, to which Genette refers in passing in his Narrative Discourse, may involve an appreciation of the design unit of the page or other aspect of composition perceived as a simultaneous whole. Thus, the global look is not only ambivalent in relation to narrative structure and understanding, but it may describe various kinds of readerly desires, impulses, or intentions, as well as different types of attention, such as the desire to contemplate the page layout as a unit of visual design, or a kind of quick look at the whole of the composition before reading it. Furthermore, there is a potential ambivalence in this concept between a global look, which can refer to different aspects of nonlinear looking and eye movement-a global look at the whole of the strip or the page, or a circular, freely-moving gaze around the zone of composition-and a type of reading that seeks to anticipate and, perhaps, to some extent also understand the narrative meanings of the work, in particular concerning the visual content of the composition. This contradiction can be avoided, however, insofar as we may be able to conceive the two activities of looking and reading as aspects of the same cognitive process in understanding comics.

Finally, it is important to point out that this chapter has been limited in its focus in that I have not considered the generic frames and publication formats that also influence, at least within some broad parameters, the representation of time in comics. One question that could have been given room here is how reading conventions, including for instance the expected time of the reading, are related to a particular genre and publication format. Humorous newspaper comic strips, for instance, are usually meant 
to be consumed speedily. They suggest a quick narrative rhythm, based on three or four panels that include a punchline. Generally speaking, popular genre fictions, such as mass-produced superhero comics, children's comics, humour strips in album series, such as Titeuf or Le Chat in France, or manga such as Dragon Ball or Naruto, invite extensive rather than intensive reading. By contrast, more intensive and in-depth reading is expected of narrative genres, where it is common to use more text, visual detail, more complicated panel relations, and create more complex characters, situations, and plots. ${ }^{44}$ The reading speed of some types of action manga may approach that of a flipbook, while the reading of complicatedly self-reflexive graphic novels, such as Chris Ware's Building Stories, is expected to take time. However, the relation between genre and the time of reading is also quite relative: the genre-related expectations are not always met, or the readers may challenge them. For instance, some popular comics, such as Edgar P. Jacobs's Blake and Mortimer series, include a considerable amount of text and visual detail, and invite the reader to spend more time in reading. Besides, many popular genres and series have dedicated fan cultures that encourage rereading and intensive reading. Thereby, a series that may have been designed for extensive reading can become something that is frequently reread or read more intensively by the same reader in another situation or a different kind of publication. The reproductions of popular strips and magazines in the book format encourage rereading while the change of the format may in itself invite the reader to spend more time with the work.

\section{Notes}

1 See, for instance, Scott McCloud's influential claim that "In learning to read comics we all learned to perceive time spatially, for in the world of comics, time and space are one and the same" (1993, 100).

2 Ware has emphasised that by allowing readers to shape the order of their reading, he wanted to explore the way in which "stories and memories are available from all sides and moments in our memories, and not really part of a continuum" http://www.tcj.com/i-hoped-that-the-book-would-just-befun-a-brief-interview-with-chris-ware/.

3 This is partly due to the privileged position that the notion of event has enjoyed as the basic constituent of narratives. See, for instance, Prince $(2003,58)$ or Abbott $(2008,15)$.

4 The distinction between fabula and syuzhet was first conceived in theoretical terms by the Russian formalists. See, for instance, Boris Tomashevsky (1965, 66-67).

5 See also Herman $(2002,214-215)$.

6 Surprisingly, however, Schüwer does not employ the narratological framework of temporal organisation in any systematic way. Surdiacourt contends that Schüwer's understanding of the story-discourse distinction is, in fact, based "on a traditional (read: structuralist) conception in which the story provides the raw material for a particular narrative representation (or discourse) and thus logically precedes this representation" (2012). 
7 The narrative present is a sense of the present moment of the story events. It is more or less distant from the time of its telling, that is, the temporal frame of the narrator's act of narration.

8 Genette derives his terms from Greek-analepsis (flashback), prolepsis (anticipation), and achrony-but these formulations are not widely used.

9 By a scene I mean an event or a situation that is unified by space, time, and the characters that are present. A scene can also be defined as the conventional equivalence between story-time and discourse-time in the given narrative segment. See Prince (2003, 85-86).

10 I find Neil Cohn's remarks in this respect to be highly relevant. He points out that "the gap cannot be filled unless it has already been passed over, making closure an additive inference that occurs at panels, not between them" (2010, 135).

11 For Robert C. Harvey, a breakdown is the process of dividing the narrative into successive panels in a narratively effective way $(1994,8,14-15$; 2005, 21).

12 Beaty also argues that the six categories could be boiled down to four basic cases: transitions that involve either a change in the subject of action or attention (subject-to-subject), a shift time (moment-to-moment), a complete change of scene (scene-to-scene), and the non-sequitur (1999, 69-71).

13 Dale Jacobs's notion that the gutter can be employed for "virtually any rhetorical end" is in line with this argument (2007, 504-505).

14 Groensteen's 'arthrology' is a neologism from the Greek arthon (articulation).

15 Postema has formulated the two-way reading of panels elegantly: "In looking at narrative panels in comics it is necessary to read back and forth in gathering the signification of comics panels. They do not represent stages or moments, but rather a continuum of possibility that remains fluid even after one has read the panel. In reading panels one's eye weaves continuously back and forth, as the meaning of one panel retroactively resignifies what was seen in the previous panel" $(2013,75)$.

16 See Baetens' convincing reading of this passage $(1989,93)$.

17 Neil Cohn's question "Where do the relations stop?" $(2014 b, 68)$ in this regard is relevant. However, the idea of "unrestrained transitions" between every possible panel in a document, and that would "overload the working memory of the human mind", remains mainly theoretical. The semantic relevance of all panel relations must be established on a case-by-case basis. See also Miodrag's defence of Groensteen (2013, 127-129, 134).

18 See Sattler on the disjunction between text and image, their interpolation of narrative and episodic memories, and their joint encoding of experiential memory in this passage $(2010,209-212)$. I would also like to thank Leena Romu for her comment on the woman's downward movement.

19 The multiframe does not have any predetermined limits, but can take various shapes such as a comic strip, a half-page format (2-3 strips together), a page, double spread, album, and a whole book (Groensteen 2007, 30-31).

20 Compare with the claim, which is perhaps revelatory of a certain antinarrative impulse at the heart of Groensteen's theory, that the principle of iconic solidarity does not have an inherent narrative purpose $(2011,17)$.

21 As in Chavanne's seminal work (2010, 18-23). See also Jesse Cohn's claim that the analysis of page layout can reveal how stories elicit the reader's desire to know $(2009,56)$.

22 Chavanne emphasises that even if the zone of composition frequently corresponds to the page, this is not always the case, and thus the notion of "mise en page" (the page layout) could be rejected $(2010,13)$. The point is relevant, 
but the emphasis on the strip as a basic unit of design has the unfortunate result of marginalising the significance of the page.

23 Peeters stresses that these four categories should not be perceived as mutually exclusive or in a historical sense $(1991,36 ; 1998,52 ; 2007, \mathrm{n} 5$; and Jesse Cohn 2007). Groensteen has complemented this model by the distinctions of regularity or irregularity, and discreet (discrète) or ostentatious layout style (1999, 112-118). The English translation mistakes the French 'discrete' as 'discrete' when it should be 'discreet', as in understated (2007, 95-101). See also Baetens' and Frey's discussion of these models $(2015,108-120)$ on the basis of the degree of correspondence between the page layout and the panel content (2015, 130-133) and Bateman's (2016) further important expansion. The relevance of this model for Japanese manga has been questioned by Rommens (2000).

24 The choice of the term 'decorative' is unfortunate on account of its normative connotations, i.e. that some element of the work is only of secondary value. Jesse Cohn points out, logically, that if the distinction between rhetorical and decorative layout "is to be of any use, it would seem, there must be some case in which we can be certain that the visual architecture of the page is not relevant to the meaning of the narrative, that it is purely ornamental. If images narrate, however, then no image can be purely innocent of narrative meaning" (2007, italics original).

25 Benjamin Widiss has emphasised the meaning of those passages in Ware's comics that, instead of (or as much as) engaging the reader fully in a sequential temporal progress, solicit seeing and "a process of association and reflection only partially dictated by narrative prompts" $(2013,89)$.

26 See also Gerald Prince's notion of the disnarrated (1992, 28-38).

27 Neil Cohn's cognitive linguistics model perceives sequentiality on this level, pertaining to the readers' comprehension of narrative meaning and conceptual information in a given unit of attention, according to the preference rules that guide readers. For Cohn, these rules dictate that: (1) grouped areas are preferred to non-grouped areas, (2) smooth paths are preferred to broken paths, (3) one should not jump over units, and (4) one should not leave 'gaps' in reading $(2013,9 ; 2014 \mathrm{a}, 6)$.

28 See Singer $(2012,57)$ for some examples of these categories in comics.

29 In McCloud's theory of time in comics, these two levels are confused. See also Cortsen (2012, 41-45) and Miodrag (2013, 118). However, it is also worth keeping in mind, as Genette points out, that diegetic time in in small segments of narrative fiction "is almost never indicated (or inferable) with the precision that would be necessary" for a detailed analysis of rhythmic effects $(1980,88)$.

30 Metalepsis is understood here in the Genettean sense of a paradoxical transgression of the boundaries between narrative levels (1980, 234-237).

31 Genette, in fact, refers to discourse-time as conventional pseudo-time and, thus, privileges story-time as a kind of real-time $(1980,33-35,94)$. The emphasis is problematic, given the fact that fiction, generally speaking, does not refer to actual events and situations, but these events and situations are created by referring to them in the telling.

32 See also Groensteen's discussion of the rhythmic function of panel frames (1999, 55-56) and the three basic ways of reinforcing rhythm in comics that conform to a regular layout: repetition, alternation, and progressivity.

33 See, for instance, Bongco, who argues that "the panel [...]graphically and diegetically unifies image and text in the comics: it forms a graphic unit which represents one moment, one instant of action in the narrative" $(2000,58)$. 
34 For Douglas Wolk, for instance, comics are "made up of a series of discrete moments" $(2007,125)$.

35 See, for instance, Eisner on the use of a full-page frame as a unit of time $(1985,63)$; Baetens and Lefèvre on the superposition of different phases of movement or the showing of different phases of the same movement by several similar figures (1993, 51-52); Hatfield on multiple images in a single panel or parsing simultaneous actions into successive frames (2005, 52-58); and Postema on the multiphase picture and panels that include visual signs from different time frames $(2013,19-20)$.

36 See, for instance, Postema (2007, 498-499) and Cohn (2007, 39-43; 2010, 131-132), on the polymorphic panel.

37 Cardinal functions are distinct from 'catalyses' or 'catalysts' that are not essential to the narrative action and the causal-chronological coherence of a narrative. See Prince $(2003,11)$.

38 See, for instance, Edward Branigan's reading of the first sixteen panels in Nick Fury, Agent of S.H.I.E.L.D. story entitled "Who is Scorpio?" (by Jim Steranko, June 1968) (1992, 81-82).

39 Groensteen has listed, among the ways in which cartoonists can accentuate a particular panel or a sequence, both formal features of the composition and the panel content, such as the panel's placement on the page, its form and size, how much of the subject is visible in the image, or a change in colouring, style, and the quantity of information (verbal or visual) (2011, 170-171). Also, frame types can emphasise narratively salient moments.

40 More precisely, Marion writes, "I would call the narrative function that which guides the viewer in gliding past the frame, and the panel function that which, on the contrary, is responsible for eliciting a fixation on the image by isolating — through various means-a continuum" (Joyce Goggin's translation in Mikkonen 2010, 82). See also Hatfield (2005, 48).

41 For instance, Lewis Trondheim and Jean-Christophe Menu's album Moins d'un quart de seconde pour vivre (1991), where only eight different images are used in 100 strips that contain four panels each. See also Groensteen on repetition as a specific form of emphasis within a regular layout (2011, 160-163).

42 See Herman's definition $(2002,213)$ : “[polychrony] includes both the more or less 'radical' types of inexactness in coding, as well as both the multiple and the partial ordering of the events".

43 See also Baetens on the insufficiency of this division in relation to the totality of the different operations that comics can employ, including duration in single panels, or the possibility of tabular or translinear connections between the panels (1998, 75-76; Baetens and Frey 2015, 106).

44 Eric S. Rabkin, for instance, has pointed out how different degrees of information intensity and representational immediacy in panels affect the speed with which we read a given frame and how this is often relative to the genre of comics $(2009,37)$. 


\section{Part II}

Graphic Showing and Style 
This page intentionally left blank 


\section{Narration as Showing}

Narration by visually showing something that evolves from one panel to another is such a common feature in comics that the importance of this mode of narration may remain unrecognized, unlike, perhaps, an unusual layout, impressive spreads, or richly detailed panels that demand greater attention from the reader. The depiction of characters in actions and situations in a world that they inhabit easily encourages a narrative response and evokes a story-like scenario in the mind of the reader. For instance, the introduction to Alan Moore and David Lloyd's $V$ for Vendetta (1989), involving twelve panels and set in 1997, first shows four panels portraying a dystopian post-war London and then juxtaposes two situations with two anonymous characters who are both dressing up and making up, or masking up, in front of a mirror. Evidently, the two characters, a masked man and a young woman, are listening to the same radio broadcast. The characters are shown in alternating panels; first, the woman preparing herself is presented in two panels, and then a man, shown from behind, appears in the next panel, after which the woman appears again in the proceeding panel. This is followed by two smaller panels of the man's hand and his mask. Finally, each character is given one panel each, the last panel including the chapter indication: "Chapter One: The Villain". The eight panels of this scene thus follow the pattern of 2-1-1-2-1-1. The verbal component of the passage does not suggest a narrative but, rather, gives some information on their world and a shared temporal frame for the characters' actions. This verbal 'track' is a radio news broadcast, reporting the weather, police surveillance, and raids on the homes of supposed members of a terrorist ring, the young Queen's public appearance, and other matters. The broadcast also underscores the duty of every man "in this country to seize the initiative and make Britain great again". The continuation of the overheard radio programme accentuates the impression of simultaneity created by the two juxtaposed situations. The images, however, carry the main narrative load. The two characters, evidently residents of this gloomy world, are shown to us in similar situations that suggest a story-like scenario, thus encouraging a narrative response. They are both preparing for an event that evening—perhaps a meeting, a date, a ball, or a masquerade—but 
we do not yet know what it may be or even if it is the same event. What increases the narrativity in this passage is the mystery of how the two situations are connected in the world of the story.

Comics usually tell a story in both words and images, but sometimes they predominantly or exclusively rely on images. The fundamental point that will be made in this chapter is that images in comics can visually articulate the narrative by showing certain things in certain ways. By showing I refer here to the use of pictures to communicate narrative meaning in front of the reader's eyes. In particular, I will focus on the presentation of characters, their bodies, behaviours, and actions that move the story forward, or at least evoke a story-like scenario.

To date, the narrative function of showing has remained implicit or cursorily articulated in comics theory. One reason for this may be that much theory on comics, seeking to define the formal essence or the basic elements of the medium, such as sequentiality, the spatial arrangement of the panels and the page, or the gutter, has deemed any considerations of visual material, for instance, relating to characters and their worlds, a matter of changing content. At the same time, while most comics concentrate on characters and their actions-showing human-like, but not necessarily human, characters in particular situations and events-this is not the only way to tell a story in this medium. Comics can take a narrative form merely by means of verbal narration. A further reason for the lack of theoretical interest in the issue of showing, as a narrative issue, is that visual showing in comics cannot usually be reduced to a mere narrative function. When an image in a comic shows a person, thing, scene, situation, or world, this may be done in terms of describing, presenting, or demonstrating instead of moving the story forward. Think, for instance, of the impressive surfacing of Captain Nemo's octopus-like Nautilus submarine in the harbour of Cairo, or the blood-smeared Mr Edward Hyde bursting into a Parisian flat in the first instalment of Alan Moore and Kevin O'Neill's The League of Extraordinary Gentlemen (1999), and how these page-size panels not only introduce us to important elements of the storyworld, but also present something spectacular in their own right. These panels function like a painting, showing a world, people, and things in terms of description, even if they are part of a story. Visual description, as in describing a character or milieu, is an important dimension in most narrative comics, both for its own sake as an aid and as an elementary part of narration. What would, for instance, be left of the appeal of Léo's popular science fiction saga of planetary romance-the three series of Aldebaran (1994-1998), Betelgeuse (2000-2005), and Antares (2007-2013) —without the visual imagination of earth-like but alien environments and strange creatures? These creatures are at the heart of the mystery of Léo's intergalactic adventures, prompting us to ask what these worlds and their habitants are like, what they contain, and how they function. Many of the larger 
images in these series create a world by describing it, thus presenting a world and its fascinating creatures for viewing.

Yet another reason for the lack of interest in the category of showing derives from literary narratology: classical models of narratology perceive showing in literary narratives as a problematic notion of mimetic presentation that equates some techniques of narration with life-likeness. For this reason, in literary narratology, the distinction between showing and telling has been frequently rejected, or modified by a gradient scale of varying modes of presentation and degrees of narrative mediation with regard to narratorial intrusion. However, in theatre, film, and comics, showing can have a more literal sense, with no relation to a narrator, and may be more easily separated from the presumed effect of verisimilitude. In these contexts, showing can be equated with the evolving performance, the moving image, or the sense of witnessing an event in front of one's eyes. This does not mean that showing can somehow represent the world directly or that the question of mimesis is irrelevant in this context; rather, mimesis becomes relevant in a different way, in and through the visual content of the images. In comics, the viewing and observing of drawn images are never 'direct'; it is always mediated at several simultaneous levels, including the level of graphic style. With the exception of photorealistic images, narrative drawings in comics bear some traces of the maker, the act of drawing, stylistic choices, and generic features-they do not simply represent something. The viewing of images in comics is also obviously indirect to the extent that pictures interact with the words that surround them, are superimposed onto them, or are placed within their space, and thus what is seen in the images becomes filtered and interpreted by words and verbal statements.

Before rehabilitating the concept of showing in the study of narrative comics, however, we need to specify what we mean by showing as a form of narration. First, it is important to emphasise that the narrative meaning of pictorial content is only an aspect, sometimes more pronounced and sometimes less, of images in comics, and not the only reason why images and visual material are presented for viewing. Second, the category of showing provisionally sets aside the important issue of the interaction between verbal and visual modes of presenting in comics storytelling. Therefore, the risk of a kind of myopic perspective in this regard is real. At the same time, it should also be emphasised that there are evident gains to be made from considering showing as a distinct narrative mode in comics. For instance, it may allow us to highlight the ways in which comics can tell a story by visual means alone. Furthermore, it offers us another means of accommodating the rich history of wordless comics, sometimes known as silent or pantomime comics, within the narratology of comics. ${ }^{1}$ In addition, it enables us to consider the narrative significance of those passages in comics where images lead the narration even if they also interact with words. 


\section{Showing and Telling}

From the writings of Henry James and the critic Percy Lubbock until the present day, an established distinction has existed between 'showing' and 'telling' in literary criticism even if there has always been much disagreement about the meaning of showing in literature. In literary fiction, the notion may be held to mean the impression that the story event is shown to the audience as directly as possible, for instance by means of dialogue or presenting a character's thoughts to be read word for word. More precisely, what can be called showing in literary narratives involves the use of narrative techniques that contribute to the mimetic illusion upon reading the text: the direct representation of the characters' speech; the aspiration of objectivity in scenic presentation; the narrator's spatial, temporal, and epistemic distance to the events told; and the avoidance of techniques of telling, such as summary and condensation. ${ }^{2}$ For instance, a dialogue scene can create a greater illusion of mimesis (imitation of real speech) than a narrative of events or a reported discourse of a character's thoughts. In the telling mode, in contrast, the narrative evokes the impression that the events are told by some agent, such as the narrator or the storyteller.

The distinction between showing and telling has its roots in the poetics of Plato and Aristotle, and especially in Plato's Republic, where narration can take the two basic forms of diegesis, which Plato calls the plain narrative, and where the poet (or storyteller) speaks in his own name, and mimesis, where the narrator imitates the characters' speech, which is thus quoted directly. Additionally, storytelling can be based on a combination of these two forms. ${ }^{3}$ Contemporary narrative theory has often employed, modified, or undermined this distinction. For instance, in The Rhetoric of Fiction (1961), Wayne C. Booth attacked the modernist emphasis on showing as the most advanced form of narration in literary art, arguing convincingly that the use of a particular narrative technique does not amount to more life-likeness or higher aesthetic value. In other words, all forms of narration have a man-made qualitythey are shamefully artificial (Booth 1983, 20). Gérard Genette's influential argument in this respect was that all forms of mimesis in literary narratives, in contrast to dramatic representation, are illusory: no written narrative "can 'show' or 'imitate' the story it tells. All this can do is to tell it in a manner which is detailed, precise, 'alive', and in that way give more or less the illusion of mimesis" (Genette 1980, 163-164). Language creates, Genette further argued, meaning without imitating (except, perhaps, for onomatopoeic words) and, consequently, mimesis in words can only be mimesis of words $(1980,164)$. Thus, the distinction between diegesis and mimesis in literary fiction becomes an issue of $d e$ grees of diegetic presentation, and it may no longer be conceived in terms of the polar opposites of mimetic showing and diegetic telling. 
A benefit of the narratological debate on the value of the category of showing is that it has illuminated the varying effects of imitation in different modes of presentation and narration. However, what can be meant by 'showing' in other narrative media and arts, such as comics, especially if they include performative and visual forms of presentation, is clearly different. While in written narratives (without illustrations), showing is metaphorical or involves types of direct discourse, showing in comics plays a central role both literally-a world is created by showing it-and metaphorically, i.e. the drawings stand for a world, characters, and their actions.

This may be stating the obvious, but a major appeal of comics is their visual style, how they are drawn, and what they show. Visual showing is a crucial component of the dynamic of the narrative in this art-it cannot be reduced to issues of panel relations, techniques of sequencing, the layout, or perspectival choices. Generally speaking, the images in comics, cartoons, and animated films are drawn in a way that maximizes their narrative meaning and function. What they show, then, is a form of telling. ${ }^{4}$ In John Leech's single-image cartoon "Substance and Shadow", published in Punch on 15 July 1843, and often regarded as the first 'cartoon' in the modern sense of the word-i.e. a satirical image in graphic form-the postures and expressions of the people seen at the art exhibition depicted in the cartoon create a marked contrast with the affluent people and idyllic scenes portrayed in the paintings around the walls (Figure 2.1). The highly caricatured visitors, the waifs and paupers in dirty rags, the many infirm and disabled, bemusedly inspect the exhibition at Westminster Hall, consisting of 'cartoons', in the sense of largescale sketches for historical murals, ${ }^{5}$ that are competing for selection by the government to decorate the new Houses of Parliament. Besides the title of the drawing, which refers to the ironic meanings of substance and shadow in the situation, Leech realizes much of his satire-a critique of government, and possibly also of artists who ignore social issues such as poverty-through graphic showing, by juxtaposing the two worlds of the visitors and the paintings.

But are images in cartoons and comics more interesting when they show (or tell by showing) rather than just describe? The question does not admit a straightforward answer. There is, as has already been underlined, so much variety in comics, and even inside a single comic in this respect. Nonetheless, a twofold argument could be made on the basis of this variety: comics can not only tell by showing, but they can also show things independently of the narrative function. Typically, the ratio between narration and description (or exposition) continually shifts within one story. It is worth noting that when Groensteen claims that for narrative drawing, showing and telling are one and the same thing, he immediately relativizes that claim, conceding that there are moments in comics when the narrative pressure is, in fact, released $(2007,163)$. 


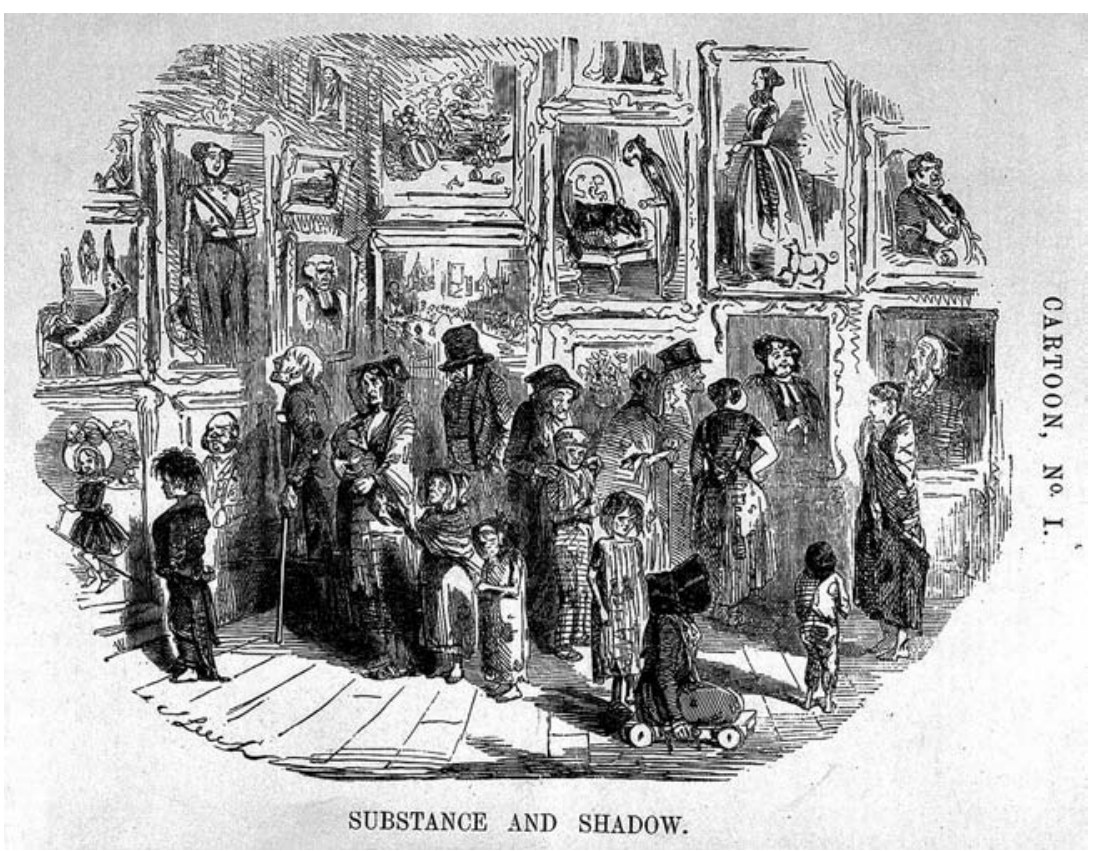

Figure 2.1 John Leech. "Substance and Shadow" (1843).

Such a release is evident, for instance, in individual panels, sequences, or larger units of composition that seek to be more pictorial, poetic, introspective, or sensual than narrative.

\section{Showing in Comics}

The focus on visual showing may allow us to better grasp the narrative mechanisms at work in comics, and, in particular, the narrative function that images play in this art form. However, before moving on to a more systematic discussion of the concept, we should reflect on the concept of 'image', more particularly on the pictorial specificity of images in comics. Most conventionally, an image in comics is expected to be a product of graphic design, that is, a drawn image consisting of lines and strokes that are applied to a two-dimensional surface. ${ }^{6}$ The comics and film scholar Philippe Marrion defines the graphic art of comics, or what he calls graphisme, accordingly, as the "configuring" use of lines, traces, colours, figures, and signs on a surface, with the intention of expression and representation (Marion 1993, 3). There are obviously some important exceptions to this expectation, and the idea that comics always involve drawn, graphic images is clearly too all encompassing. Comics 
may, for instance, incorporate blank colour panels, mere text panels, collage images or photographs, and they may even be wholly based on photographs (so-called 'fumetti') or computer-generated images. In the past two decades, computer technology, in particular, has transformed the making and conception of images in comics, and it can be difficult or outright impossible to distinguish between what is drawn and what is computer-designed in today's comics.

My focus here is on graphic images that invite a narrative response by showing an event or a situation that involves characters. Whether they are actually drawn or not is beside the point. For our next example of showing, let us consider H.M. Bateman's famous "One Note Man" (14 December 1921) wordless comic from the artist's "Man Who" strip series, ${ }^{7}$ which was published in the magazines Tatler and Punch in the 1920s and the early 1930s. "One Note Man" is a story four full pages in length that relates a day in the life of a musician who plays a clarinet-like wind instrument in a large symphony orchestra. On the first page of the story, we see the main events in what seems to be an ordinary day for the musician: he wakes up, takes a bath, has breakfast, smokes his pipe, practises playing, takes a bus to the concert hall, and chats with some of his colleagues before the concert. At the concert, his task is to play a single note, which seems to put him under much strain (Figure 2.2). We then see the man sneaking out of the rest of the concert, going back home, having dinner, smoking his pipe again, and getting ready for bed.

The humour of this story has various sources, one of which is the idea, also conveyed by the title, that the protagonist's musicianship consists of playing just one note. This does not seem like much in comparison, for instance, to the violinists, whose labours are also shown in close-up images. Another source of humour is the manipulation of narrative rhythm during the story, especially in the concert scene when the man plays his note. The various small events in the man's life, from shaving to smoking his pipe, which are shown before and after the concert, are given roughly equal space in the story, usually one panel per event. The panels show us that the man spends most of his waking hours in non-musical activities that neatly divide his day into a familiar and steady pattern. During the concert, however, the narrative rhythm
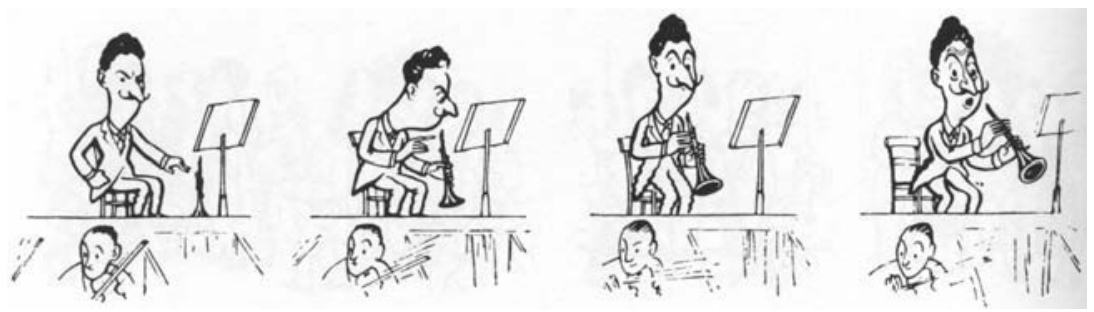

Figure 2.2 H.M. Bateman. “The One-Note Man” (1921). 
slows down considerably. The concert scene represents over half the panels in the story, while the playing of the note, and the immediately preceding and following moments, take almost one page. Therefore, the carefully timed moment of terrible intensity is humorously contrasted, through means of narrative rhythm, with a life that otherwise seems extremely monotonous.

Perhaps the most important source of humour in this story is nonetheless the visual content of the images. First of all, the presentation of the man's continuous action from panel to panel, and the pattern of following that is thus established, allow us to think of these strips as an evolving story. Second, the depiction of the man's expressions, postures, and body language, and the inferences that the reader can make about the changes therewith, move the story forward. The way in which the man's expressions are drawn suggests that he is rather content with himself and his life. For most of his day, his facial expression changes very little, beyond the need to yawn in the morning and at night. The man looks unwaveringly content and relaxed, and his posture is also confident as he walks along the street. The only striking exception to his demeanour of calm is when he actually plays the note. This is clearly reflected in his face, posture, and body language, which are shown in a series of four panels: (1) the man reaches for his instrument with a concentrated look on his face; then, (2) bends towards his music note stand, and very carefully follows the notes on the page; next, (3) he is shown with both hands placed on the instrument, and his eyes opened wide; and finally, (4) he is depicted with his eyebrows acutely raised and his mouth open, ready to play the instrument. After the note, the man slumps down dramatically, almost collapsing with his chair, while holding his chest in exhaustion-he has given his all for that crucial note (Figure 2.3).

To understand the story and its humour, the reader must be able to attend to changes in what is shown in the image sequence. The title of the story does not add much to the reader's understanding; rather, it repeats what can already be appreciated by following the visual content of the images.
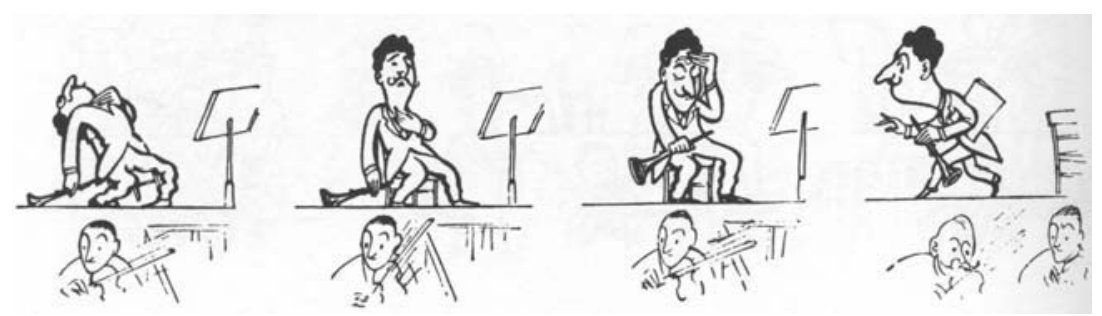

Figure 2.3 H.M. Bateman. “The One-Note Man” (1921). 
How, then, should the narrative showing of visual content be integrated into comics narratology? At least two approaches are readily available. On the one hand, to better understand the medium-specific qualities of showing in comics, we can revisit the theory of showing, or monstration, in film narratology, and modify the concept for our purposes. On the other hand, we can analyse the synthetic role of the continuing character as a means of connectivity and coherence. The musician in "The One-Note Man", who can be seen in each of the fifty-eight unframed panels of this comic strip, builds a sense of continuity and coherence between the panels by allowing space, time, and action to continue throughout the sequence. Next, we will turn our attention to the concept of showing in film studies. The issue of continuing characters will be tackled in the next chapter.

\section{The Concept of Monstration}

The theory of showing in cinematic narratives is based on André Gaudreault's ground-breaking narratological work from the 1980s and $1990 \mathrm{~s},{ }^{8}$ where he redefines Plato's concept of mimesis as monstration. ${ }^{9}$ For Gaudreault, a film narrative is the product of a linking, often complex and layered, of the two basic modes of narrative communication: narration and monstration (Gaudreault 2009, 7). In this context, narration does not refer to delegated narrators in film, who tell the story in voice over-voice-over narrators are, in fact, only a limited source of narration in films, or a kind of "sub-narration" (Gaudreault and Jost 1999, 53-54); rather, it denotes techniques of editing that permit individual shots to be articulated together as a narrative (Gaudreault 1987, 31). Narration, therefore, means sequencing, a process in which individual frames are turned into narrative units, i.e. shots. Editing also enables temporal modulation in films, that is, a change of temporal frame allowing, for instance, the unfolding events on the screen to be conceived as occurring in the past. In contrast, Gaudreault defines monstration as a mode of narrative communication that is limited to a sense of the present in the evolving events $(1987,31)$. More precisely, monstration is

a mode of communicating a story, which consists of showing characters (in English, monstrance) who act out rather than tell the vicissitudes to which they are subjected. Monstration could thus be used to replace the term "representation," which is too specific, too compromised, and far too polysemic.

Gaudreault $(2009,69)$

Theatrical monstration is therefore the product, at least in part, of the characters in dramatic action, that is, the performance of characters acting out the narrative (Gaudreault 2009, 37, 40). Monstration, moreover, 


\section{2}

includes two aspects of communication: mimetic and non-mimetic diegesis. Mimetic diegesis is a form of staged narrative where the characters depict the action through their physical actions and behaviour, while a non-mimetic mode of narrative communication may involve various forms of talking and quoting, such as conversation-in other words, "all cases in which words, speech, and verbal discourse are used within a staged performance to tell a story" (Gaudreault 2009, 71).

The different modes of verbal and visual narration are assigned to theoretically distinct agents in Gaudreault's model, which he terms narrator and monstrator (show-er of images). These agents are responsible for verbal and visual narration, respectively, and are set in a hierarchical model of narrative levels where they are both subordinate to a narratorial agent at a higher level of cinematic narration, which Gaudreault calls the 'mega-narrator', or 'grand image-maker'. This is the plural agent who is responsible for modulating all manifestations of "theatrical language" in film: mise en scène, set design, lighting, and acting (Gaudreault 2009,72 ), and coordinating the various sources of expression, including images, sounds, words, written materials, and music. However, according to Gaudreault, in collective productions such as theatre or cinema, the narrator is a more fleeting theoretical construction with less welldefined borders than is the case in literary narratives.

\section{Monstration in Comics}

In comics studies, Philippe Marion has applied and modified Gaudreault's concept of monstration in his theory of "graphic enunciation", published in the reworked version of his doctoral dissertation as Traces en cases (1993), which has been one of the key texts in French-language comics theory since its publication. Sharing Gaudreault's interest in narrative agents, Marion uses 'enunciation' to refer to the act and circumstances of producing an utterance in comics-with utterance meaning here, for instance, a strip, a page of a comic, or a narrative. ${ }^{10}$ For Marion, the very heart of enunciation in comics is their graphic material, the graphic trace (Marion 1993, 9-10). It is this graphic quality of images, so Marion claims, that always makes the drawings in comics, to some extent, opaque signs, at least in comparison to cinematic and photographic realism. Thus, by letting us see the trace of the graphic act-the signs of the graphic performance-showing in comics never has the same figurative transparency or the same transitivity as in cinema (Marion 1993, 36). Moreover, unlike a photograph, the graphic images in comics constitute the material that they present.

Generally speaking, enunciation in this sense is distinguishable whenever readers become aware of any signs of the writer or cartoonist's subjectivity. This may also occur whenever readers find themselves confronted by comic-book conventions as such, from the observation 
that "this is comics", acknowledged by self-reflexive uses of basic comic-book devices, such as layout or techniques of sequencing, to "I am reading a comic book (a strip, a graphic novel)", such as when a comic self-consciously reflects on its materiality or the publication format. ${ }^{11}$ In principle, any noticeable stylistic changes may increase the reader's awareness of enunciation. This can be a pronounced graphic line and trace, unusual colours (choice of colour palette, contrast, tone, shade, and so on) or chiaroscuro, types of foregrounding and backgrounding in the image field, or a number of other choices that pertain to the visual register and graphic design, and which may highlight the subjectivity of the source of narration.

However, to focus on narration and monstration as instances of enunciation makes it harder to see the narrative function of showing in its own right. In fact, what really seems to matter in Gaudreault and Marion's notions of showing is the identification of different kinds of narrative agents, to whom they ascribe responsibility for different aspects of presentation and who allow them to develop the theory of a higher-level narrator. The approach is in line with the Genettean model of narratology, where narrative mediation is equated with the narrator concept, i.e. all narratives supposedly have narrators, but it distances us from the question of narration as showing.

We will return to the question of narrative agency in comics in more detail in a later chapter. Here, it is sufficient to note that the category of showing, understood in the sense of characters acting out the story through a comic's visual content, can be distinguished for analytical purposes in comics. More precisely, showing can thus be understood to refer to certain aspects in the narrative drawings of comics that are presented for viewing, specifically characters and their actions, behaviours, and situations in a particular world, and that inspire a narrative response from the reader.

The way that comics narrate by showing is exemplified in a section in Bastien Vivès's graphic novel, A Taste of Chlorine, that relies on visual showing alone. The lack of words in the story is partly motivated by the protagonist's solitary visits to a swimming pool and the depiction of the act of swimming itself. At the same time, showing through images is also very effectively used in this work to convey mental and physical states. One particularly striking wordless scene, which lasts for eighteen pages, takes place towards the end of the story, when the protagonist decides to swim the whole length of the swimming pool underwater. As he approaches the end of the pool, he suddenly notices above him a woman with whom he had become acquainted earlier, a woman whom, to his great disappointment, he had not seen for a long while. The man seemingly tries to grasp the woman's leg, but either fails to do so or pulls back since he realizes that she is actually someone else. Whatever the case, at the same time, he gulps, sinks back, and runs out of air. The man starts 
to swim furiously upwards, at times evidently astonished and terrified by the distance. At the end of the scene, he finally comes to the surface, gasping for breath.

The various mental states that the protagonist goes through in this scene are depicted visually, by showing his changing facial expressions and postures. Thus, following the salient details of how the scene is acted out, we are able to follow the character's determination when diving, excitement in seeing the woman above him, his struggle in trying to catch her, surprise at being suddenly out of air and still so far from the surface, desperation in trying to reach surface, and total exhaustion when he hangs on the edge of the pool at the end of the scene. There, resting and looking towards the reader, he then seems to see something, but what that might be is not shown. Thus, the image of the gaze is used for a specific rhetorical effect: to stress the importance of the moment, and the event, and to increase the sense of the story's open-endedness.

There is obviously some latitude in our verbal interpretation of this scene and the character's actions, in particular relating to the man's emotional state. The precise meaning of the facial expressions and bodily postures portrayed in the scene may be hard to pinpoint. Perhaps the man is not so excited by the woman or the sense of challenge as he is simply curious and daring. Or, possibly, he is more disappointed with himself than surprised by not being able to catch her, fearful of what may happen instead of being desperate. Nevertheless, the exact terms that we choose to describe his mental state in this scene are to some extent irrelevant. What matters is the necessity of understanding the acting out of the scene and making narrative sense of the many visual cues for his overall mental state.

The concept of showing allows us to discuss the tremendous variety in comics with regard to the degree in which they tell a story by presenting characters in situations in narrative drawings. The differences in this regard relate not only to the ratio between narration by showing and telling by words, but also to the extent to which non-visual objects, such as propositions and states of mind, may be expressed visually, through such means as facial expressions. Generally speaking, such differences reflect both the choices and styles of individual cartoonists and authors and also generic expectations, particular traditions of the art form, and the reader's capacity to detect mental states from external signs. Typically, action comics and mainstream manga rely more on the image alone to convey narrative information.

In more text-oriented comics, the use of strategically chosen wordless panels or sequences is a common technique for achieving particular narrative effects. Wordless narrative drawings may, for instance, effectively alter narrative rhythm (a wordless panel being an indication of eventless time or the passage of time, for instance), accentuate the visual aspects of an event or a place, prompt the reader to look for the salient visual 
details or create a particular dramatic effect. Such functions are realized, for example, in the strategically placed wordless and textless panels in Raymond Briggs's graphic story of his parents' married life, Ethel \& Ernest. A True Story (1998), where dialogue carries the main narrative load. Among the wordless elements of this work is a page that contains a picture of Ethel and Ernest's wedding on which is partly superimposed another picture of the couple sitting on "the lovers' seat" in Fairlight Glen, Hastings in 1930. The juxtaposition of the two images shows that the couple had got married and travelled, possibly on their honeymoon, to this site on the south coast of England. Thus, set in relation to the honeymoon picture, the 'wedding' photograph functions as an index of an event and an easily recognizable scenario that does not have to be related by words.

While the wedding picture condenses time into one image, two other wordless panels in this story accentuate the visual and physical aspects of the represented experience. These include a panel which shows Ethel struggling with a large white sheet that has been drying on a clothesline in the garden, and another panel that shows a doodlebug (the V-1 flying bomb) flying over the cartoonist and his father, who have flung themselves to the ground in a field. In both of these panels, the object (the sheet, the bomb) that is presented for viewing, as well as the sound of the bomb, extends over the frames of the panel. The breaking of the frame heightens the object's size and speed, and the uncontrollability of its movement, both from the perspective of the characters and the reader. On the one hand, the relationship between the moving object and the characters in the image, and in Ethel's case also her facial expression and posture, suggests a strong emotional state. Ethel appears to be as much marvelling at the sheet as she is struggling to keep it in place-the sheet's movement is also associated with the white birds flying in the distance-while the speed of the doodlebug, and the shadow that it casts over the cartoonist and his father on the ground, increases the sense of fear in this scene. On the other hand, the objects are thus presented for viewing as something to be marvelled at and, potentially, as something that allows the reader to share the characters' sensations and emotions. Finally, the last wordless scene of the book, comprising four panels, relates Ernest's lonely death, contrasted with the couple's long life together, in which dialogue had played such a vital role. Here, the lack of words is a feature of the storyworld and Ernest's lonely situation, not just an element of dramatic effect or a means of description.

Although the wordless panels in the otherwise dialogue-centred story involve important events in the narrative, they are not uniform in purpose. While they involve scenes in which the main characters act out "the vicissitudes to which they are subjected" (Gaudreault 2009, 69), they also serve a descriptive function and invite the reader to share the character's visual experience. 


\section{Graphiation and Graphic Style}

Marion argues that the notion of monstration alone is insufficient to describe the medium-specific features of graphic showing in comics. This is because monstration in comics, so he claims, lacks the figurative transparency or transitivity ${ }^{12}$ that it has in cinema $(1993,36)$. Consequently, Marion developed the concept of graphiation, to describe the kind of enunciation that is typical of comics. Graphiation pertains to the graphic and drawn qualities of comics (lines, traces, and graphic design, used in both images and lettering) when these elements call attention to themselves. The notion of graphiation, therefore, focusses on those elements in comics that are not simply narrated or shown, but are only traced, or that have a traced, graphic quality that takes precedence over other qualities, such as the content that they present for viewing or narrative function. Graphiation may concern both drawn images and words, in particular when their graphic traces (empreinte graphique) draw attention to themselves as markers of the cartoonist's subjectivity. ${ }^{13}$ Therefore, by emphasising the drawn and written quality of graphic lines, the concept creates a unified perspective for viewing both text and drawing.

The focus of the concept of graphiation, then, lies on graphic style and, in particular, a specific stylistic effect or use: the autoreferential function of graphic design, where the graphic trace points to itself and, thus, functions as a marker of subjectivity, in particular, of the cartoonist's subjective style. This effect may be conceived simultaneously as a kind of artistic expression and a form of readerly attention where the graphic material draws attention to itself instead of moving the story forward. In other words, the notion casts light on the fact that in analysing narrative comics, we may be able to differentiate not only between what is shown in the image and how that something is shown, but also between the graphic act of showing and drawn traces that do more than just narrate and show or do not yet (or no longer) narrate and show.

The distinction between graphic showing and graphiation, while useful in highlighting the fact that not all visual content in images shows or tells something, or that graphic styles have different degrees of autoreferentiality, nevertheless, raises a terminological and conceptual problem concerning whether the concept of graphiation can do something different or better than the notion of graphic style. While the concept of graphiation remains popular in French-language comics theory (but less in actual analysis and research), it also has been convincingly argued that the scope of this concept is unclear, in particular regarding the extent to which it overlaps with the notion of style. ${ }^{14}$ Here, I choose not to integrate the concept of graphiation into the narratological approach to comics, the reason being that it is far from clear that we need a new term, let alone the construction of another narrative agent which 
would be responsible for the act of graphiation and would then require narratological attention of its own. As I hope will become evident in the course of this book, the notion of graphic style provides us with sufficient means for making useful distinctions between styles ranging from the maximally ostentatious, or autoreferential, where subjective expression may be highlighted, perhaps even at the expense of the objects shown in the images, to styles that aspire to maximal transparency and objectivity.

\section{Conclusion}

By concentrating on the narrative function of the visual content of images, I have demonstrated above why the concept of showing is useful for the narratology of comics. The concept allows us to better conceive and emphasise the visual aspects of narration in comics, i.e. the way in which comics rely on visual means of storytelling by showing characters engaged in a situation, behaviour, or action in some world. If what Genette calls narrative tense looks at the relationship between story time and discourse time, voice concerns the question of 'who speaks?' (i.e. 'who is the text's narrative voice?') and mood the question of 'who sees?' (or, 'where is the centre or the focus of perception?'), and then the category of showing invites a two-sided question: 'what is shown and how?' We can also reformulate this question from the reader's perspective and in relation to narrative comics in particular as 'what is shown in the image that inspires a narrative response?' and 'how does what is shown in narrative drawings make us relate one panel to another?'15 These questions are not just about image content-'what is this image about?'-but pertain to the narrative function of that content.

The issue of graphic showing, again, raises the question of medium specificity. With regard to the visibility and materiality of the graphic trace, there is perhaps no crucial difference between comics, cartoons, and animated films, except that in an animated film the trace is perceived as moving. In general, comics, cartoons, and animation privilege maximally narrative drawings that feature expressive physical gestures and easily identifiable features of physiognomy, action, and situation. The drawn quality of the image modifies the way in which something, such as a character's body, expression, or engagement in situation or world, can be shown. Nevertheless, showing in comics also has some medium-specific elements. Such elements include, perhaps most importantly, the narrative function of the sequence and the page layout. Thus, the frame is both a structural and expressive device-a feature that is also common in single-image cartoons-which can, in comics, also exert influence over the narrative process by separating the panels and setting a pattern for their reading and viewing. Another distinguishing factor in 
comics in this respect is the way in which the field of vision relates to the potential visual field outside the picture frame. While comics regularly reveal, in retrospect, that something important has been left out of the previous frames, the off-screen space seems to play a much more important and consistent role in animation and cinema than in comics. This may be because in comics the image is expected to focus on what is most salient in the evolving event, situation, or a place, and that any references to contingent but excluded fields of vision need to be clearly motivated. ${ }^{16}$

\section{Notes}

1 For the history of wordless comics see, for instance, Groensteen (1997, 1998), Gravett (2013, 34-53), and Kunzle (2001).

2 For a more extended discussion of these techniques, see Klauk and Köppe (2014).

3 See the third book of Republic (3.392c-398b).

4 See also Groensteen, who has defined the laws of narrative drawing in comics as those of anthropocentrism (the privileging of the character as an agent of action), synecdochic simplification (the leaving out of everything that is not necessary for intelligibility), typification, expressivity (maximal expressivity in the characters' faces and bodies), and rhetorical convergence, i.e. the narrative image, by all means of composition, colour, and framing, obeys the imperative of optimal legibility (1999, 190-191; 2007, 162).

5 Before the popularity of satirical cartoons in the printed press, the term 'cartoon', or cartone in Italian, had meant a finished preliminary sketch on a large piece of cardboard.

6 Baetens and Frey argue that "the fact that the story is less told or shown than drawn is what defines the difference between comics and graphic novels and storytelling in other media" $(2015,165)$. I do not think that such a distinction is useful, just because, as Baetens and Frey also write, "(l)ines display a story world in which the act of drawing cannot be separated from the drawn result" (ibid.). Moreover, the distinction between showing and drawing does not suggest a very sound basis for distinguishing between comics, cartoons, and animation.

7 It is widely known that the idea of the Albert Hall sequence in Alfred Hitchcock's original version of The Man Who Knew Too Much/ was derived from this cartoon.

8 Gaudreault draws not only on the Genettean model, but also on Albert Laffay's earlier "protonarratological" narrative theory of cinema outlined in Logique du cinema (1964).

9 Gaudreault's (2009) basic argument is that diegesis (the poet or the narrator speaks in his or her own voice, recounting of the events) and mimesis (theatrical form of presentation, imitation of an action) are not opposite categories in Plato and Aristotle since mimesis can be seen as a form of diegesis in Plato and diegesis as a form of mimesis in Aristotle.

10 In the linguist Emile Benveniste's definition, enunciation is the act of producing an utterance (énoncé) or mobilizing a language for the speaker's own use (1970, 12, "un acte individual d'utilisation").

11 I borrow Gaudreault and Jost's phrasing of cinematic enunciation $(1999,49)$.

12 Transitivity refers here to the way in which the contents of an image refer to a direct object-in contradistinction to a reflexive relation (in graphiation), 
where the material of the image refers to itself or, in other words, the direct object is the graphic line itself.

13 See also Baetens $(2001,150)$. Dürrenmatt $(2013,160)$ compares graphiation to the poetic function of language since it obliges to view the graphic gesture in itself, including the visual aspects of the alphabet.

14 See, for instance, Groensteen (2011, 92), Dürrenmatt (2013, 159-167). Baetens claims that Marion's distinction between monstration and graphiation is convincing, but is critical of these notions, nevertheless, with regard to what he sees as Marion's monolithic understanding and the biographical trappings of the graphiateur concept $(1998,39-40 ; 2001,152-3)$.

15 Verstraten formulates a similar question about narration through shots in film: "who or what is being shown and how are they being shown?" $(2009,56)$.

16 The issue of "hors-champ" (the field of vision outside the panel frame) has not been much theorised in the field. Baetens and Lefèvre, however, have coined the term péri-champ and discuss the diverse ways in which a comics panel can refer to the virtual visual field outside its frame, and activate it (1993, 26-36). See also Groensteen (2007, 119). 


\section{Character as a Means of Narrative Continuity}

The continuing presence of a character or a group of characters in a sequence of images, acting out a situation, or participating in an event, is possibly the most conventional feature of narrative comics. The ability to follow an easily identifiable character, such as Tintin, Astro Boy, Tank Girl, or "One Note Man", in evolving action from panel to panel, maintains a sense of continuity and coherence in a narrative sequence. In fact, the continuing character is such a common element in comics that it has sometimes been perceived as a defining characteristic of the medium. ${ }^{1}$ This, however, is taking the argument too far: While characters are undoubtedly central to narration in comics, comics are able to tell a story without the presence of one entity whose situation, action, or experience endures for the whole length of the work. Cartoonists, similar to literary authors and filmmakers, often shift attention away from one character to another, perhaps even in the course of a single scene. Sometimes juxtaposed or contiguous images are more relevant for storytelling than continuing characters, and it is always possible that a story establishes a scene or describes a milieu instead of a character in action and, in fact, a narrative can be present in a comic without a character being shown at all (see chapter on characterisation). For instance, a focus on the inner workings of someone's mind, i.e. the thoughts and emotions of a character or narrator, can make a narrative. In more experimental comics, frustrating the reader's ability to follow a visible character may also be the desired effect. That said, however, for a narrative comic to have no continuing entity that is repeated or which the readers may follow, presents a real challenge to the comic's sense of causal continuity and narrative coherence. Here, rather than being seen as a guarantor of the quality of a narrative or an attribute of a good narrative, cohesion simply refers to causal cohesion in a sequence of panels. ${ }^{2}$

Consequently, one might say that continuing characters are not a requirement for narrative comics; rather, they are a central convention that increases narrativity in any comic. In terms of the narratology of comics, the issue of the continuing character brings into play various key considerations and concepts. On the one hand, the depiction of a character 
in a sequence of images gives the reader access to the story through a clear unit of attention that can be followed. The continuing character can thus be conceived as an aspect of focalisation, or more precisely, of the focalised (the object of the perception). On the other hand, a continuing character allows the reader to gradually construct a person-like entity engaged in some action or situation and have a sense of the story content: The narrative is about a particular character or group of characters. Narrative events, and the experiences that stories highlight, usually revolve around people, or human-like characters, and their actions, perceptions, and experiences. Furthermore, the presentation of the mental states, thoughts, feelings, and experiences of sentient beings, which many narratologists regard as key characteristics of narratives regardless of the medium, suggests the presence of some agent who acts, feels, and thinks and whose actions or thoughts and perceptions can be followed.

Therefore, characters are simultaneously salient features of narrative comics at various levels, as person-like agents, as a means of narrative transmission, and as units of attention that move the story forward and allow readers to follow it. In narrative comics, salience in this sense involves the question of how something is shown, including the means of narrative drawing, layout, framing, and the juxtaposition of panels, stylistic choices, and the interplay between visual and verbal narration. However, what is considered salient cannot be merely reduced to the formal features of narrative comics. Narrative salience is also an expectation that readers have of the way in which stories are constructed and told and the outcome of their active interpretation. One expectation in this regard is that characters are allocated different roles and significance in fiction, i.e. there are main and minor characters. Another significant expectation is that the depiction of person-like agency and goal-oriented action will enable the reader to become imaginatively engaged with the narrative. This point is commonly made in narrative studies of film. The film scholar Murray Smith, for instance, claims that our 'entry into' the narrative structure of fiction films is mediated by character (1995, 17-18). We will return to Smith's argument at the end of this chapter. Similarly, many literary narratologists hold that the representation of an experiential agent is a minimal requirement of narrativity and our primary access to the narrative. The mere depiction of some character's action can say much about their intentions, thoughts or emotions, and experience.

Here, I consider a character present throughout a continuing situation, event, or action as a basic tool for building narrative continuity and coherence in comics. The point in thus focusing on and isolating the question of the synthetic ${ }^{3}$ role of the character, i.e. their continuity-building function, from other considerations pertaining to characters, such as focalisation, characterisation (characters' person-like qualities), or the representation of speech and thought, is to better cover the visual and multimodal means of connectivity employed in comics, and thus further 
develop the issues of connectivity and showing discussed in the previous chapters. This will also raise the issue of the relationship between visual and narrative saliency, ${ }^{4}$ which can not only overlap but can also be distinguished from each other in terms of what is central in the image (the content that is shown) on the one hand, and what is important in the image in terms of the narrative as a whole on the other. Verbal narration, in captions and dialogue, can by itself select for the reader those characters, entities, or objects that are to be tracked and followed, for instance by creating a sense of a character's continuing consciousness or a continuing conversation. Verbal narration may also specify how the situation or the protagonist's actions and movements should be understood, emphasise the salience of something, or point out, especially in first-person narration, that the narrator's inner experience is more important than what he or she may be perceiving (as shown in the images). In the next sections, however, the focus will be on the visual showing of continuing characters and the reader's spatial attachment to them.

\section{Match on Action in Comics}

The repetition of the same character in a panel sequence creates a visual bridge between the images. This basic convention can be clearly observed, for instance, at the beginning of stories. In the first instalment of Saga (2012), by Brian K. Vaughan and Fiona Staples, the cover image already establishes the two protagonists, Alana and Marko, as important figures in the story. The first panels of the first scene, which depict the birth of the couple's daughter Hazel, then confirm that the story is about these two characters. Both Alana and Marko are shown in close-up images that focus on their emotional states and intimate relationship. The first panel is an extreme close-up of Alana, who is clearly suffering, while the second panel, an establishing image, shows her lying on a table with someone between her legs, helping in what is evidently a childbirth scene. The two characters' emotional engagement with one another is then portrayed by an image and reverse image sequence where we first see the horned man Marko looking tenderly at Alana and commending the winged woman for her beauty, and then see Alana, suffering labour pains, looking less fondly back at him and responding with a sarcastic comment (Figure 3.1).

The visual bridges between the panels of this scene are based on recurring characters and the sense of their continuing action. Match on action is the most common continuity editing technique in filmic narratives and extremely common in comics as a means of panel transition: to match different shots with continuing action, the basic purpose of which is "to allow space, time, and action to continue in a smooth flow over a series of shots" (Bordwell and Thompson 1986, 231). In this technique, one shot cuts to another shot portraying the action of the subject in the 


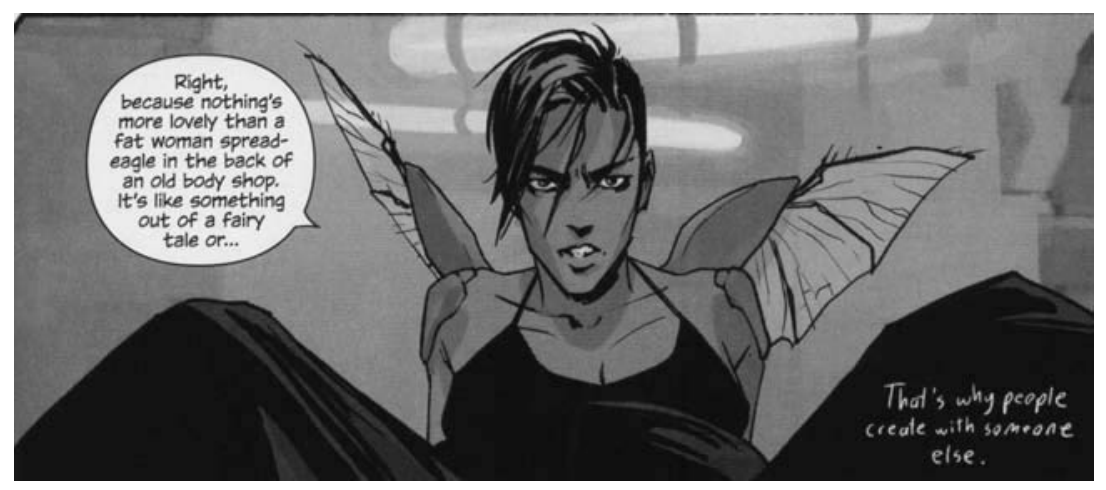

Figure 3.1 Brian K. Vaughan and Fiona Staples. Saga @ 2014 Brian K. Vaughan \& Fiona Staples.

first shot. Thus, the character's (or characters') activity creates a visual bridge between the gaps-that is, the shots-and conveys a sense of continuity in the scene. The effectiveness of this technique relies on its ability to suggest a simultaneous sense of temporal and spatial coherence. This is the reason why David Bordwell and Kristin Thompson also argue that match on action is a particularly powerful continuity editing strategy: "If an action carries across the cut, the space and time are assumed to be continuous from shot to shot" $(2008,250)$. We could further add that match on action as it typically involves a character or characters, provides film narratives and narrative comics alike with a centre of experience that can function as an additional dimension of continuity, that is, as an experiential frame connecting the narrative units. ${ }^{5}$ This is because the character imports a sense of subjective experience to the image, unlike in a match cut (or graphic cut) where bridging between shots, or images in comics, is based on graphic repetition and spatial matching between particular objects, shapes, spaces, or other visual aspects of the composition, suggesting thus an analogy of things seen or, possibly, a visual metaphor. ${ }^{6}$

One key difference with regard to match on action in comics and films is the so-called $180^{\circ}$ rule, which can be seen as the very basis of continuity editing in film narration, ${ }^{7}$ but which plays a more limited role in comics. This rule dictates that in a film the scene's movement-a person walking, people meeting, a car racing along a road, someone swimming laps in a swimming pool-is assumed to take place along a clear-cut vector or axis of action. In other words, the axis of action determines a semi-circle or $180^{\circ}$ area where the camera can be placed to present the action. The rule is not absolute in films-it can be broken for specific effects without necessarily undermining the coherence of the shot-but 
avoiding placing the camera beyond the line of action is a powerful means of reinforcing the impression of the continuity and coherence of action. The rule guarantees that the participants in a scene have a consistent eyeline direction and the same left to right relationship, and thus a shared notion of what may be off-screen, and it creates the impression that their movement has a continuous sense of direction.

In comics storytelling, the axis of action can also function as an important structural principle. Traditional comic strips, where the characters and their action are systematically seen from a steady angle somewhere to the side of the action, follows this rule strictly, thus providing the reader with the experience of stage-like action. However, the lack of perspectival changes in such strips likens the viewing experience to theatre rather than modern cinematic fiction. In modern comics that may alter the angle, focus, and width of the perspective, the logic of direction in movement also often respects the culturally bound rule of depicting forward movement from left to right (in a panel as on the page) whereas movement from right to left typically denotes return. ${ }^{8}$ For instance, in Rob Davis's graphic novel The Motherless Oven (2014), where the story involves a considerable amount of walking and running-the characters peregrinate in the streets, walk to school, or escape from school, fleeing from the police, the provost, or their mechanical and sometimes monstrous parents-movement is invariably depicted from left to right (from different angles) with very few exceptions. When the characters appear, unusually, to be moving from right to left, they are forced to run (anywhere) for their lives or save each other, or seek shelter from a rain of knives (literally a rain of knives). The changed direction of movement, thus, stresses the exceptional situation. In turn, panels that show characters moving towards the reader allow us to concentrate on their facial expressions, eyeline, and dialogue. In contrast, the movement of characters away from the reader tends to reinforce different perspectival effects, such as establishing an over-the-shoulder perspective, i.e. sharing the character's perspective, or emphasising distance, such as the changing distance between characters. ${ }^{9}$

In today's longer comics and graphic novels, such as The Motherless Oven, which employ alternating perspectives, the logic of narrative space and the direction of action are seldom challenged by changing the angle across and around the axis of action. However, at the same time, the axis of action is a highly flexible and relative notion in this context. ${ }^{10}$ The angle can move around the characters and the scene without undermining the sense of logic in the narrative space, the direction of movement, or the relative positions of and distance between the characters. It is, in fact, a kind of default expectation in much of the storytelling in contemporary comics that every panel in a scene changes the angle and field of vision by moving around the characters, their action, or the whole scene. Such constant shifts and contrasts of 
perspective are often employed, for instance, for scene-building in conversational scenes.

Another medium-specific means of connectivity through the characters in comics is to place and orient the characters in the panel in such a way that their gaze points to the next panel, thereby prompting the reader to look the same way. This may be particularly effective in cases where the order of the panels diverges from the most conventional forms of linearity. ${ }^{11}$ Awareness of a panel connection may thus be inbuilt into the representation of the characters through the direction of their movement and gaze.

In this double spread from the fourth instalment of The Walking Dead, by Robert Kirkman, Charlie Adlard, and Cliff Rathburn ("The Heart's Desire", 2005), the story is dominated by a large, panoramic panel which stretches over both pages and 'bleeds' to the sides of the pages and which is surrounded at the top and bottom by smaller panels (Figure 3.2). One of the top panels also partly extends over two pages, and the order of reading in the upper strip is reinforced by speech balloons that are superimposed onto the gutters. The double spread portrays the moment when the occupants of the prison, who had just been engaged in a leadership dispute, realize the approaching attack of the living dead from another prison block. The living are thus forced to join forces to protect themselves. Here, the protagonist, Rick Grimes, is shown from different angles in three corners of the double spread, as

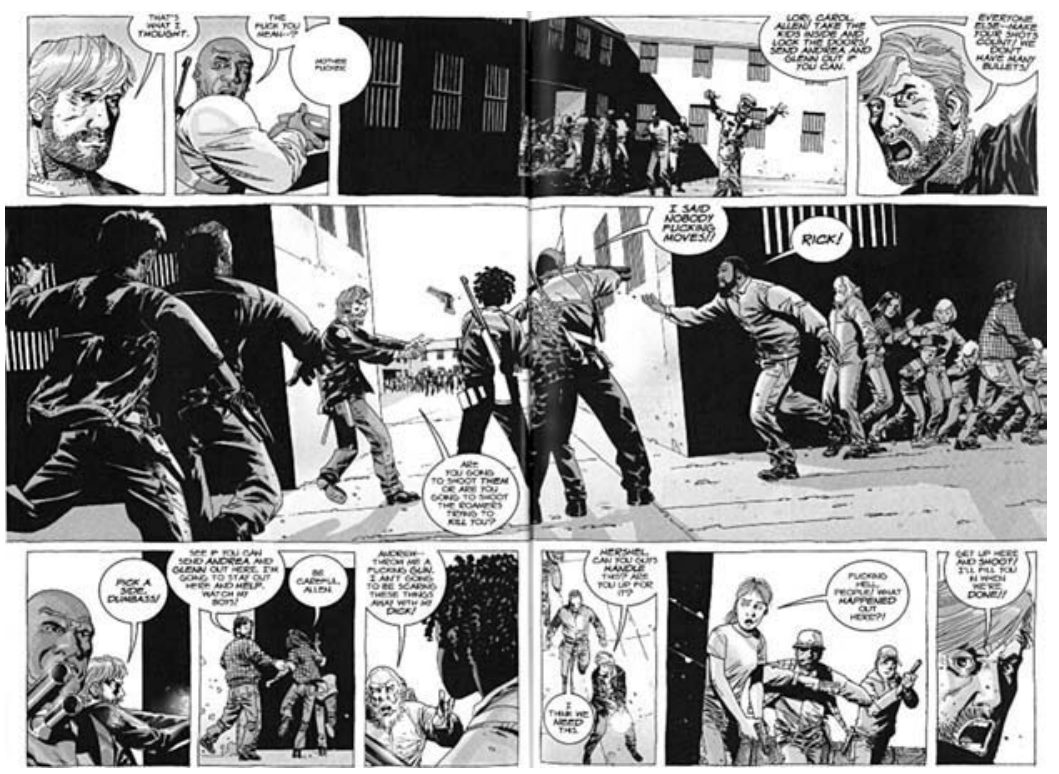

Figure 3.2 Robert Kirkman, Charlie Adlard, and Cliff Rathburn. The Walking Dead4 (2005) (C) Robert Kirkman. 
well as from above and behind (turned towards the right) in the panoramic panel. The constantly changing perspectives and positions do not undermine continuity in the passage; on the contrary, they add to the sense of fast-paced action and decision-making in the scene.

The double spread is unusual to the extent that panels or tiers of panels rarely extend over one page in The Walking Dead series. However, the arrangement is not so extraordinary given that in The Walking Dead, page layouts are dynamic and constantly change according to the action and the dramatic situation. The panel sequences in this double spread are highly typical of the series in terms of their perspectives, i.e. how the angle of vision constantly shifts from panel to panel around the characters of the scene, with the perspective sometimes focusing on a particular character, or approximating their perspective, before panning out from them once more.

The flexible, dynamically changing angle of vision around the characters and their action, irrespective of the $180^{\circ}$ rule, does important narrative work in many genres and works of contemporary comics. Unlike narrative films, comics are unconstrained by the need to avoid contradictions in the depiction of space and movement in moving images. On the contrary, they may exploit their necessarily discontinuous form to enhance the effects of a moving perspective without causing jarring effects in narrative development and the sense of space. The characters' changing position and orientation, furthermore, suggest a sense of continuing action or an ensuing event.

What is extremely similar in narrative comics and film, however, is the role of recurring characters in creating a sense of narrative coherence, and the fact that discontinuity in such characters' action makes scene changes more obvious. In other words, a character shift, ${ }^{12}$ which may occur either by addition, subtraction, or the complete change of major characters across panels, can indicate that a particular panel transition in fact represents a transition from one event, scene, or episode to another and from one narratively salient element to another. ${ }^{13}$ Therefore, the change of focus on a continuing character allows the reader to make inferences about transitions in narrative emphasis and in the larger narrative structure and context. ${ }^{14}$

\section{Continuing Movement}

Repetition of the same character in a sequence of panels can suggest narrative continuity when the sense of action and event is weak or the images depict no action. The showing of a character in stasis (that is, someone who is motionless and unengaged in any obvious activity) can serve as the description of a character or a mental state as well as the means of establishing a scene or a situation. ${ }^{15}$ Moreover, in contemporary comics such as in The Walking Dead, it is quite common for the images to focus predominantly on the presentation of dramatic situations by showing the characters' faces, positions, and gestures from different angles, instead of 'directly' depicting their movements or actions. 
In traditional superhero comics, action and dialogue are often found to alternate more frequently, but in The Walking Dead series, despite the centrality of action, the images rarely portray several phases of an ongoing movement or action. Typically, while the characters in The Walking Dead are engaged in a physical confrontation or are escaping zombies-both very frequent situations in this series-they are also engaged in a conversation. ${ }^{16}$ Although the action scenes in this series show aspects of the physical action, the focus lies on the characters' facial expressions and bodies, their gestures, positions, and situation in relation to one another and the depiction of their engagement in a conversation.

When comics portray movement and phases of action, or when a character is shown moving from panel to panel, the focus on the character's spatio-temporal path can serve as a means of continuity in quite specific ways. This technique is effective for the same reason as match on action in films: the depiction of phases of movement creates simultaneous spatial and temporal connections. In addition, however, in the showing of a character's movement, two levels or articulations of sequentiality also coincide: the character's imagined mobility and the sequence of the panels. Both of these levels of sequentiality help to mask or negate the discontinuous form of narration present in comics. Movement in itself is a way of connecting story elements and adding new elements at various levels: The phases of movement can create a rudimentary narrative, while moving characters also introduce the reader to new places and characters, thus giving the reader new reasons to follow the story. Furthermore, the illusion of movement provides the story with a visual logic that helps direct the reader's attention and the order of reading. The characters' forward movement-typically moving from left to right-reinforces the forward flow of the reading experience from panel to panel, strip to strip, from top to bottom, or in manga from upper-right corner to left and from the back of the book to the front. Potentially, a character's movement can also contribute to the reader's sense of curiosity, surprise, and discovery on the verso page after the page has been turned.

In order to better understand the function of movement in narrative comics in this regard, we should consider briefly the importance of stories of motion and movement for the development of narrative comics in the nineteenth century. As much research on nineteenth-century comics has shown, in the latter part of the century, the narrative art of comics developed greater continuity between images through the depiction of motion and action. For instance, rather than presenting separate stages of a story or an event, accompanied by captions explaining the gaps in the action between the illustrated scenes, images in sequence were increasingly conceived as phases of a continuous action (Gunning 2014, 41). This development was, in part, inspired by advances in motion capture in photography and cinema. ${ }^{17}$

Let us consider a few examples of the depiction of movement from the era to illustrate this important historical change. In John Tenniel's 
early comic strip for Punch, "How Mr Peter Piper Enjoyed a Day's 'Pigsticking' (Near Burhampoor, Bengal)" (1853), the eight panels of the story describe the different phases or events of a rather ridiculous and anti-heroic hunting trip. In these panels, the protagonist, Mr Peter Piper, first attempts to spear a wild boar and falls off his horse. He then stumbles over a family of crocodiles in some bushes, meets a tiger in the same bushes, climbs back on his horse, seemingly frightened by the predators, catches some piglets on his way back, rides triumphantly away with his pitiful game, and finally boasts of his achievements to his friends while having a hearty meal outdoors(Figure 3.3). Even though the panels are thus connected, beyond the captions, by both the recurring character, his movements, and the theme of hunting - the protagonist is moving in each image, and in the first panel we also see motion lines to emphasise this fact-the sequence lacks a strong sense of continuity between the various stages of action. Rather, what these panels depict are representative situations, such as major mishaps or revealing moments of self-deception, as the day progresses. While many aspects of the story rely exclusively on visual narration, there is no attempt made to capture ongoing action or movement from panel to panel.

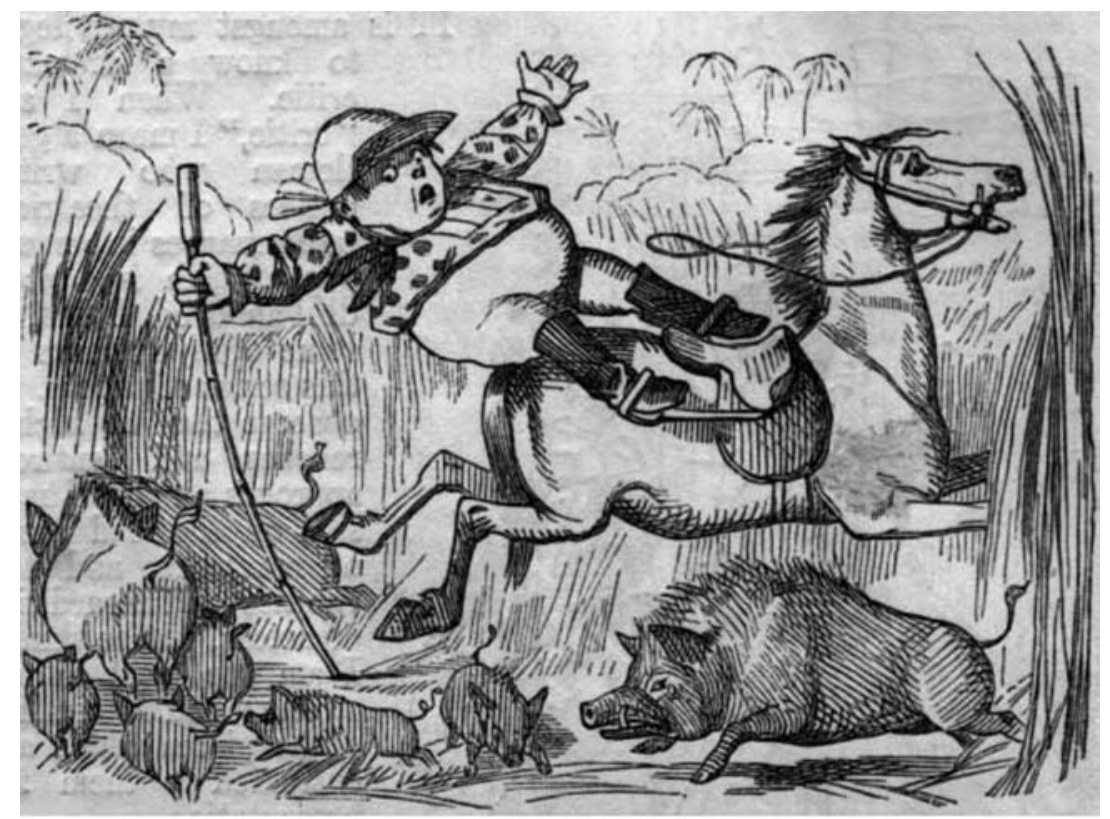

Having arrived at the 'Hunting Ground', Mr. Peter Piper tries to take a 'first spear' and fails.

Figure 3.3 John Tenniel. "How Mr. Peter Piper Enjoyed a Day’s 'Pig-Sticking”” (Near Burhampoor, Bengal)' (1853). 
Various means for depicting a sense of continuous action and movement nevertheless already existed at this time, and Tenniel was also aware of the alternatives. Two decades before Tenniel's stories in Punch, the Swiss artist Rodolphe Töpffer had developed a sense of continuing movement and physical action through a lively drawing style and the juxtaposition of image content, using such devices as character gestures implying a particular motion or a gradual movement (a chase scene, for instance) and modifications to the formal features of composition. The latter included the use of a dynamic, changing panel size (narrower panel, shorter duration, for instance), and framing-references to what is left out of the image can suggest causal links in movement, for instance.

It is important to note that these historical developments in the portrayal of movement were neither linear nor unidirectional. In another comic that Tenniel drew 2 years later, a story entitled "Mr Spoonbill's Experiences in the Art of Skating", which appeared in Punch in three instalments in February and March 1855, he chose a range of options for depicting movement. In this comic strip about Mr Spoonbill's embarrassing skating experience, we first see how the inexperienced protagonist has skates put on his feet in what is called "excruciating torture", then goes through a "variety of eccentric movements", falls down several times, and then, "blind with enthusiasm", skates onto dangerously thin ice and falls through into the water, from where he is fortunately rescued (Figure 3.4). Finally, he is depicted struggling back home, where he attempts to warm himself up. While some panels show representative moments of action, such as when a "vulgar" man puts the skates on his feet and, in the following panel, conducts him onto the ice, other panel sequences of the story, especially when the protagonist falls on his back or when he falls through the ice, show closely linked phases of continuous fast movement. Thereby, Tenniel's story reveals both an interest in the representation of continuous action and movement and experimentation with the visual connections between the panels with regard to the character's action.

The situation is no different in the comics of today to the extent that the depiction of continuing movement is an option in storytelling, but the impression of a continuing character engaged in an action or situation, or just being present, is a dominant convention. In some popular genres, such as superhero action comics, or war comics along the lines of the British series Commando, scenes of continuing physical action are one of the main means by which the story moves forward. In contrast, in many contemporary forms of autobiographical comics and graphic novels, physical action and movement play only a limited role as the main focus of narration is on the author's experience, thoughts, and emotions. Nonetheless, in many contemporary comics characterised by their depiction of physical action, such as The Walking Dead, it is remarkable 


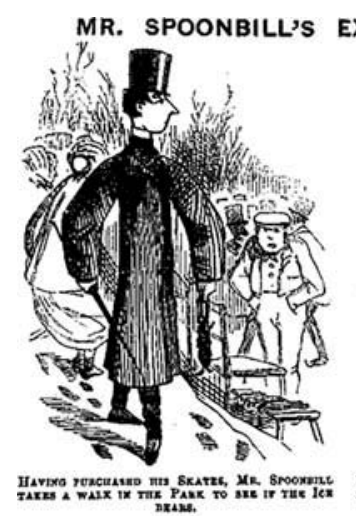

EXPERIENCES IN THE ART OF SKATING.-PART I.
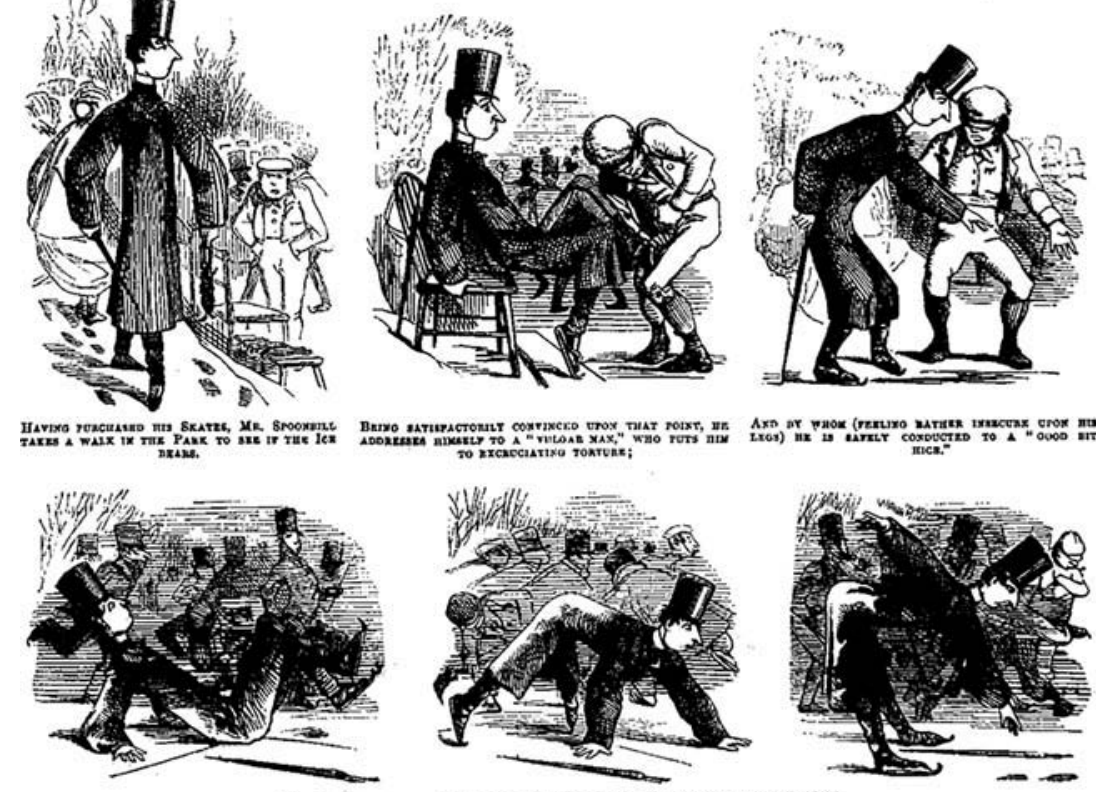

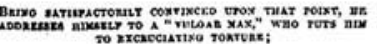

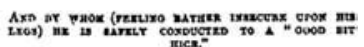
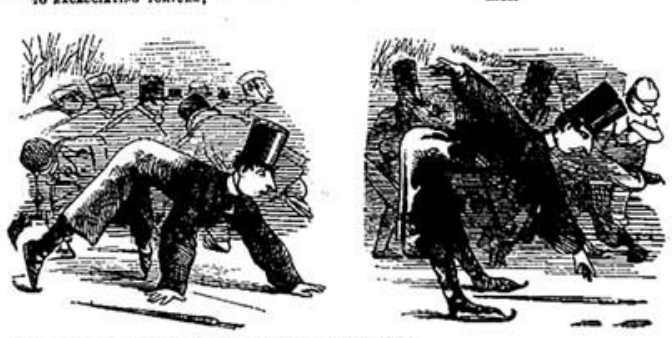

Figure 3.4 John Tenniel. "Mr. Spoonbill's Experiences in the Art of Skating” (1855).

how movement and action are implied without focusing on the different phases of movement. The rapidity of action, the quickness of succession and the complexity of the event are conveyed, perhaps most importantly, by concentrating on the expressive force of the character's face and body language. However, what have changed since Tenniel and the nineteenth century are the techniques of perspective-taking and layout, which are far more versatile today, and the various visual signs and symbols of movement and action, which have become conventionalised. In the past two centuries, the medium of comics has developed an impressive repertoire of techniques, devices, and visual symbols for portraying movement within a single panel or across a sequence or group of panels. These include motion and speed lines (lines that indicate the direction, form, or speed of movement), ribbon paths (lines or swaths of a light shade or colour that show the path along which a character or object has moved), and impact flashes (symbols that indicate sites where movements are initiated or terminated), ${ }^{18}$ blurring or streaking effects, the 'sound effects' of movement where onomatopoeia shows the direction of motion or the speed of movement, as well as superimposed images that depict various positions or parts of the movement. 


\section{Visual and Narrative Salience}

The effect of the characters' narrative salience may be reinforced by verbal narration, for instance, first-person narration, and also by visual cues. In Gunther Kress and Theo Van Leeuwen's visual grammar, salience is one of the basic means of composition in images (the other two basic factors being information value and framing). In this model, the salience of particular elements in an image can be emphasised through various formal features and cues, including the placement of the elements in the foreground or background, or their size, and the use of colour, tone, focus, perspective, overlap, and repetition (2006, 61, 183, 212-214). With regard to images in comics, Teresa Bridgeman has listed similar formal features that help the reader create a sense of salience for particular elements in the composition: depth of field, degree of iconicity (iconic or cartoony vs. a realist style), drawing styles, colour, panel limits/framing (close-up vs. panorama), composition (possible scanning patterns within the image), composition across page, page layout, and panel size (Bridgeman 2013). The character's placement in the foreground of the image from panel to panel increases the likelihood, although does not guarantee, that readers keep this character primed as an agent. Page layout, similarly, can be used to distinguish between primary and secondary information in a scene or ongoing narrative passage. Any significant variation in layout requires appraisal of what is taking place in the space of the composition. Importantly, the panel itself, which presents something for viewing, is also a unit of attention, ${ }^{19}$ and therefore, what is chosen to be depicted in a panel can be expected to be worth the reader's attention. Finally, repetition of visual content alone can draw attention to a particular figure, action, or behaviour.

Comics can use all these visual and compositional cues to bring the main character and his or her action into better focus. In this respect, however, it is important to note that the impression of visual salience in the image, for instance, due to the angle or focus in the image, or the placement of someone or something in the centre foreground, may or may not be reaffirmed as narratively salient by the surrounding panels or the narrative context. In other words, the visual salience of an element in a panel is not defined by the reader's evaluation of its relevance for the narrative as a whole.

Let us consider the relation between visual and narrative salience in an example that self-consciously tackles, and at some level also negates, the convention of continuing characters. In Martin Cendreda's one-page story "I Want You to Like Me", which consists of sixteen panels, ${ }^{20}$ the speaking characters and the spaces shown in the images change constantly, while what is said in the speech balloons, also including one thought balloon, suggests an ongoing, coherent discourse. From the outset, the story seems to lack internal consistency at the visual 


\section{2}

\section{Graphic Showing and Style}

level: characters, situations, and places keep changing, and we have no notion of whether these situations have any temporal connection. The lack of continuing characters creates various unusual effects. One of them is that the sense of narrative coherence in the sequence relies heavily on speech and the coherence of the sentences as a continuing thought, monologue, or conversation. The continuing speech suggests a shared idea and emotion for the changing characters, that is, the desire to be liked, accepted, or adored by someone whom the speaker likes. Another effect of the evident discrepancy between the characters and what they say is that by preventing the reader from following a continuing character, the story thus manages to illuminate the importance of that convention. One effect of the lack of continuing characters is that, facing the impossibility of following anyone, the reader may be tempted to search for visual clues for other connecting objects, details, or the milieu, in order to evaluate whether there might be more implicit forms of connectivity in the story beyond the ongoing discourse. In this attempt, a careful reader is perhaps rewarded by discovering that the first and the fourteenth panels actually depict the same two characters and the same scene: an artist painting on an easel and conversing with someone who is looking at his work. Once this panel-to-panel connection has been established, the reader may then be encouraged to go further on the same interpretive path. However, whether there are other translinear visual connections is much less evident. One option for such a connection is the potential visual analogy between the artist's easel, already shown twice, and the cartoonist's drawing board, the effect of which may be further reinforced by various other images or frames within the panels in this story (a computer screen, graph, blackboard, billboard, mirror). Furthermore, the angle of the dark-haired man in the last panel perfectly replicates the cartoonist's position in the earlier panel. These men also have quite similar features, thus implying the cartoonist's self-parodic reflection on wanting to be liked, through his work, by his audience. Yet again, in tracing such metaphoric connections the reader makes manifest the default expectation of being able to follow a continuing character.

\section{Spatial Attachment and the Process of Following}

I have discussed above some of the ways in which the recurring, continuing character-in action, movement, or stasis-functions as a means of connectivity between panels, in particular by creating a sense of temporal and spatial continuum through an experiential centre of attention. Similarly, in the study of narrative film, scholars have formulated models for analysing the ways in which characters 'filter' the story for the viewers. In this regard, Murray Smith's theory of spatial attachment in film and patterns of alignment and Rick Altman's medium-independent notion of following-units and following-patterns have particular potential 
for the narratological understanding of characters in comics. Both of these models stress the importance of the process of following, that is, the spatio-temporal path that a particular character takes throughout the narrative, as the audience's main means of access to the story. For Altman, the process of following, marked by the narrator's activity in selecting something or someone to be followed, usually a character or several characters, is a basic feature of all narratives, regardless of the medium. This premise seems particularly relevant in the context of narrative comics and the history of their development. Smith's approach, however, has an additional advantage for the narratology of comics in that his comprehensive account of narrative mediation through characters integrates the questions of following and focalisation into a suggestive whole.

One of the key terms for Murray Smith is alignment, which refers to a level in the spectator's imaginary engagement with characters, ${ }^{21}$ and which describes "the process by which spectators are placed in relation to characters in terms of access to their actions, and to what they know and feel" (Smith 1995, 83). In other words, alignment considers the way in which the viewer's attention is restricted to character knowledge, such as visual and aural knowledge that is more or less congruent with that available to the characters (Smith 1995, 75). The process of alignment involves, more precisely, two interlocking levels: (1) the spatial attachment to a character in the course of a scene or several scenes-i.e. the process of following a character-and (2) access to the character's mind-i.e. the processes by which the spectator may come to understand how a character perceives a situation, and/or reacts to it emotionally. Smith calls these two basic structures of alignment spatio-temporal attachment and subjective access. ${ }^{22}$ They are conceived as distinct functions, while, at the same time, they "interlock to produce a pattern or structure of alignment" (Smith 1995, 144).

The central insight in the idea of alignment, and one that clearly sets Smith's theory apart from literary narratology, is that the filtering effect of such alignment, jointly produced by these two interlocking functions, cannot be reduced to the question of perspective (or focalisation) as literary narratology defines it, even if these notions are closely related. In contrast, the concept of spatial attachment stresses the importance of characters' spatio-temporal paths for visual narration. The process of following a character is important, since it provides the spectator with an entry into the narrative. Perceptual alignment is only one dimension of this pattern. Spatial attachment to a character entails many other features, such as the character's action and behaviour, engagement in an event, and way of relating to others and the world. Thus, spatiotemporal attachment in film narration, similar to narrative comics, establishes a relation between the audience and the character where the spectator (or the reader of comics) may follow the actions of a character 
or a group of characters, witness the actions of other characters when they are in proximity to the focalised figures, and create a sense of the evolving event through the character's spatio-temporal path.

Let us think of an example that makes the significance of the convention of spatial attachment, and the accompanying subjective access to a character's mind, evident. In the first instalment of Sandman, entitled Sleep of the Just (by Neil Gaiman, Sam Keith, Mike Dringenberg, and Malcolm Jones III, 1989), the beginning of the story suggests a complex, multi-focus, spatio-temporal attachment to the characters. In the first two pages of the story, set in 1916, we see Dr John Hathaway arriving at Roderick Burgess's mansion in Wych Cross, England and then meeting $\mathrm{Mr}$ Burgess. On the third page of the story, four equally sized smaller panels are superimposed onto an image of the gate of Roderick's mansion, which thus serves as a kind of overall frame for the panels. The images within the frame introduce new characters and places, suggesting potential story paths that may be followed: Ellie Marsten in Toronto, Daniel Bustamonte in Kingston, Jamaica, Stefan Wasserman in Verdun, France, and Unity Kinkaid in London. The four situations are connected to the main narrative by the theme of dreams and dreaming, and through Mr Burgess, whose waking dreams, so we learn, are about the power and the glory and of death. On the next page, the story refocuses on events at the mansion, from where Hathaway has left. The alignment is now with Mr Burgess and a new character, his son Alex, who is shown with his father. What follows is a nocturnal scene, an unsuccessful occult ritual conducted by Mr Burgess. After that, alignment and focus shift again. The four characters who were introduced earlier are given three panels each that reveal more of their respective situations. This is then followed by another shift in alignment and a simultaneous change from external to subjective perspective, where we see Mr Burgess and Alex entering a room from the perspective of someone in the room who is not shown (later we learn that it is Dream). The subjectivity of the perspective is affirmed by the eyehole-type framing of the panels, the changed colouring of the scene, and the first-person narrative voice that we can read in thought balloons superimposed onto some of the these panels. Moreover, Mr Burgess evidently talks to and looks at that someone whose subjective vision we share and whose voice and thoughts we hear but cannot yet see.

Several more shifts of character, location, and action are to follow in remainder of the first instalment of Sandman, while some of the spatial attachments of the beginning have soon run their course. Hathaway's suicide, for instance, will shortly close up one potential path to follow. However, at the time the readers are introduced to the (as yet) unknown subjective perspective, they may ask themselves where the story is going and which of the many characters and situations is the main focus of the story. There are at least seven potential units of following at this 
point: Hathaway, Burgess (and Alex), the four characters who are given a perfectly equal amount of space-Ellie, Daniel, Stefan, and Unity-and the anonymous subjective viewpoint. Thus, the beginning of Sandman poses questions about the direction of the story and the reader's spatial attachment to the given characters: how are all these characters, situations, milieus, and paths connected? Who or what situation will emerge as the focus of the story? Here, by steadily multiplying the number of characters and potential structures of alignment (or following-units), the beginning of the story exploits the expectation that the reader needs to be able to follow a particular character or a group for the narrative to really begin. At this point of the story, it is possible that all these characters and their situations, as individuals or as a group, may offer the reader a potential point of spatial attachment.

From the reader's perspective, one might say that alignment with someone in the story involves the recognition that a character or a group of characters has narrative salience rather than mere visual salience. This recognition is achieved by the representation of the character's actions and behaviour, and/or consciousness, and is verified in a larger narrative unit in relation to other characters who are given less space and attention. Significantly, alignment with a character is also derived from the way in which the characters are shown and how the story hinges on their actions, perspective, and mental life. The continuous showing of a character plays a crucial role in creating a sense of spatial attachment in comics even if direct representation (or enactment) is not limited to the visual channel of the medium. At the beginning of Sandman, the characters' names are mentioned, and what they say or think also necessarily affects our understanding of who we should follow. Nevertheless, spatial attachment is created here by means of visual showing; there is no competing or accompanying level of continuous verbal narration in the form of dialogue or narratorial captions that could do this. ${ }^{23}$ If the character's actions are recounted by another character within the diegesis, either verbally or visually through a flashback sequence, then narration, as Murray Smith also suggests, can be said to establish an embedded attachment (ibid.).

From the model of spatial attachment, we can derive a typology of alignment patterns that also indicate some new possibilities for the development of a diachronic (historical) narratology of narrative mediation in comics. We may presume that most narrative comics, as is the case with narrative films, set up patterns of alignment between the extremes of continuous single attachment, where we follow one protagonist throughout the whole story, and multiple attachments, where narration "successively traces the distinct spatio-temporal paths of many different characters" (1995, 146). Rick Altman's conception of narratives, similarly, is in terms of following-units that form following-patterns (series of following-units) ranging from the basic forms of single-focus 
narration to dual-focus and multiple-focus narration and their alternation (Altman 2008, 26-27). The beginning of Sandman keeps all these three options consciously open. If such formal solutions for continuity building and narrative filtering were compared in a large body of comics in a particular genre or a period, this might allow us to detect historical changes, trends, and conventions in alignment (or following) patterns in the medium. More specifically, this might allow us to study historical changes in the association between forms of attachment and access to the character's mind, i.e. to identify the degree of subjective access that is entailed by attachment to a character. Similarly, a narratological history of storytelling in comics could investigate the emergence and development of parallel but medium-specific associations, formal patterns and alignment, and their wider implications for the medium.

\section{Conclusion}

What matters from the perspective of narrativity and narrative coherence in much storytelling in comics-in first- and third-person narratives alike-is that a character or group of characters, and sometimes other objects or the milieu, such as the landscape or cityscape, reoccur in the panels. Visual repetition thus builds a sense of continuity and coherence between images by allowing space, time, and action to continue over a series of panels. The showing of a continuing character from one panel to another, either engaged in a situation or action or simply present in some scene, increases narrativity in any sequence of images. We may surmise that this 'synthetic' quality in continuing characters and their actions, i.e. their use as a means to connect panels in a narratively meaningful and coherent way, functions through the reader's expectation of being able to follow an experiential centre of attention. The depiction of movement, or indicators of the character's movement and action, such as changing positions, can further strengthen the sense of connection between the images. With regard to the characters' gazes, the means of connectivity may be derived, for example, from aligning the perspective with a particular character, showing an exchange of looks between characters, showing a character looking at something and then revealing what is seen, or by positioning a character close to the picture frame.

In order to ensure that continuing characters provide temporal, spatial, and experiential connections between the panels, they are usually given high visual and narrative salience, or grounding, in comics. At the same time, it must be emphasised that neither this salience nor the way in which characters may direct narrative development are based solely on the formal means of showing the same character from one panel to another; rather, they are also 'psychological' in nature. Characters are worth the reader's attention, perhaps predominantly, because they are sentient beings who have anthropomorphic features. The mere depiction 
of a character's action or movement, for instance, already provides the reader with much information about the character's experience, intentions, and perception of the world. We will return to the questions of perspective, and the way in which characters may be constructed like people (their 'mimetic', person-like quality) in detail in later chapters, but before that we must develop the issues of graphic style and narrative agency.

\section{Notes}

1 Comics, Colton Waugh claims, usually have "a continuing character who becomes the reader's dear friend, whom he looks forward to meeting day after day or Sunday after Sunday" $(1991,14)$. Waugh, thus, refers to the readers' emotional engagement with 'mimetic' (person-like) characters with whom they already have become acquainted.

2 See also Henry Morgan's argument that the character "gives the image sequence its semantic cohesion and it is around the character that the storytelling is organised" and, further, that "it is the character [...] who allows the reader to understand the sequential logic, both in temporal and spatial terms" $(2009,35)$. Teresa Bridgeman argues cogently that the downgrading of the function of the experiencing centre is a threat to the narrativity of the text as a whole (2013).

3 See James Phelan's definition of the synthetic dimension and function of characters in literary fiction $(1989,2-3,9,20-21 ; 1996,29-30)$. The synthetic (character as artificial construct or plot device), the thematic (character as idea), and the mimetic (character as person) dimension can coincide in varying degrees, or be more or less foregrounded-their relations are determined by the narrative progression where some potential may or may not be realized.

4 These two may overlap and complement each other. See, for instance, Kruger (2012) on how visual salience in narrative films, such as the placement of certain elements in the centre of the image or shot, can be different from narrative salience, i.e. how something is shown and how certain elements are significant for the understanding of the narrative as a coherent whole.

5 Other forms of continuity editing include eyeline match, graphic match (or match cut), cross-cutting, establishing shot, re-establishing shot, narrative diegesis, shot-reverse shot, and cut in (Bordwell and Thompson 2008, 235-236; Magliano and Zacks 2011, 1491). Saraceni compares repetition between panels to a match cut $(2001,171-173)$.

6 In comics, graphic cuts can connect different scenes, but they seem less common than in films. One reason for this may be that longer ellipses of time can be easily précised in captions. By contrast, comics lack many cinematic devices for indicating a longer ellipsis, such as fades, dissolves, and wipes.

7 See Bordwell and Thompson $(2008,231,234)$.

8 See, for instance, Guaitella $(2003,523)$ on how Hergé's characters, who retrace their steps, are always shown to move from right to left.

9 The different connotations of direction in the depiction of movement in comics should obviously be examined in a larger corpus of examples to be able to say anything more general.

10 Compare with Kukkonen (2013b, 47-48) who points out that the $180^{\circ}$ rule is often violated in dialogue scenes in comics.

11 Chavanne $(2010,206-207)$ has shown how the figures' placement, organisation, and orientation in the panels can aid the technique of boustrophedon 
(the arrangement of alternate strips of panels in opposite directions) or unusual movements from right to left.

12 I am borrowing James E. Cutting's term that he has used in reference to continuity editing and narrative discontinuity in movies $(2014,70)$.

13 Some film studies, which draw on behavioural data, also confirm the importance of match on action as a major indicator of a scene and salience and, consequently, how discontinuity of action is a strong predictor of the viewers' perception of event boundaries. See Magliano and Zacks (2011, 1510).

14 Compare with James E. Cutting, who argues that continuity is a hybrid concept in films, partly psychological and partly physical-and can be broken down to the basic parameters of location, character, and time-and that the recognition of a scene is also psychologically determined, i.e. based on the psychological impression of what counts as an event $(2014,69-71)$.

15 See, for instance, Scott Bukatman's discussion of the significance of stasis, and the investment in stillness and the sculptural, in Hellboy comics (2014).

16 Baetens and Frey argue cogently that it is "a persistent misunderstanding to believe that the visual string of a graphic novel shows the successive parts of an action unfolding in time, as if the graphic novel was offering a selection of shots from a sequence of a virtual movie" and further that "What it [graphic novel] shows is in the first place a series of variations of the face. Even if graphic novels do tell stories, their first concern is not infrequently the portrait of the characters and the multiperspectival representation of their bodies" $(2015,176)$.

17 Kunzle argues that framing, already in the 1890 s, served the representation of movement, for instance by broken frames (1990, 368-369). See also Bukatman (2006, 2014).

18 For the definition of ribbon lines, motion lines, and impact flashes, see Potsch and Williams $(2012,15)$.

19 See Groensteen (2007, 53-57) and Neil Cohn (2007, 42, 2013, 56).

20 Published in Kramers Ergot, a series of anthology-style books of comic art edited by Sammy Harkham (Oakland, Buenaventura Press, 2006).

21 The two other levels are recognition (the spectator's construction and individuation of character based on a set of textual elements) and allegiance (the moral evaluation of characters on the basis of the values that they embody) (1995, 82-85).

22 By attachment, Smith means "the way a narration may follow the spatiotemporal path of a particular character throughout the narrative, or divide its attention among many characters each tracing distinct spatio-temporal paths" $(1995,142)$. By subjective access, he refers "to the way the narration may vary the degree to which the spectator is given access to the subjectivities- the dispositions and occurrent states-of characters" $(1995,142)$.

23 Smith points out that the decisive characteristic of what he calls direct representation in films is the absence of a mediating level of narration in the form of dialogue or voice-over $(1995,182, \mathrm{n} 11)$. He does not seem to consider the possibility that verbal narration and visual means of spatial attachment can also be contrasted with each other. 


\section{Graphic Style, Subjectivity, and Narration}

The question of subjectivity in comics is vast given the multiple senses of the word. To begin with, we can distinguish between two basic dimensions of subjectivity in graphic narratives, that of the author (or author function) and the character (or a narrator). The cartoonist's subjectivity can be detected in the use and combination of stylistic conventions such as the graphic line, lettering, colour, or the spatial organisation of the page. Traditionally, graphic style, the way of drawing comics, has been seen as a kind of signature of the story's creation, the image bearing the signs of its making. ${ }^{1}$ However, style as the maker's mark is only one dimension of the various aspects of style in comics. The visual style of comics can also be a largely genre- and format-related issue, shaped by a particular culture and production of the comics in question, with few traces of an individual maker. Think, for instance, of the popular Disney comics or the Manga industry where the cartoonists are expected to conform to a highly recognisable style. At the same time, it is not unusual today that the cartoonists make their mark by changing styles from one piece of work to another or even within a single work. The division of labour between the writer, the cartoonist, the colourist, the letterer, and the like introduce further complexities in this regard. The writer's style can be distinguished on the basis of linguistic and literary choices, the cartoonists are known for their visual style, the colourist for the use of certain kinds of colours, and so on. Thus, graphic style can also be viewed as the product of artistic cooperation.

Comics have a variety of devices available for presenting a character's subjectivity. These include perspectival techniques, narrative voice (manifest as external/internal, explicit, implicit, in legends and balloons), the presentation of dialogue and thought (as speech and thought balloons), the technique of spatial attachment or following (as sentiments and thoughts are revealed through action in a sequence of images), and other means of visual showing such as facial expression, gesture, body language, gaze, and the character's position in the image in relation to other things that are shown. Furthermore, a number of combined visual and verbal signs, such as metaphoric images and pictograms (emanata, symbolia) that mark thought, emotion, reaction and attitude, 
or onomatopoeia, such as interjections, can offer access to a simulacrum of the character's mind. Likewise, various aspects of spatial articulation, such as framing, sequencing, breakdown, page layout, and tabulation, can emphasise the attribution of mental functions to particular characters. For example, changes in the frame shape and size can accentuate a character's mental and emotional experience, and local changes in the visual style, such as blurry images, can indicate that a certain passage is a subjective mental image, such as a fantasy, dream, or memory. ${ }^{2}$ We will return to many of these devices in more detail later in relation to the issues of focalisation and the representation of speech and thought.

The traditional expectation regarding the relation of these two subjectivities, that of the author (or the cartoonist and the other creators) and the character, is that they are distinct, one belonging to the actual world of the story's making, and the other to the world of the story. However, as I will show in this chapter, it is possible that the interaction between these dimensions becomes significant in the story, especially in cases where a comic subverts the expectation of a unified style or closely associates important stylistic features with a particular character's mind. The question of the relation between two or more authorial subjectivities arises especially in cases where the writer and the cartoonist are different individuals with distinguishable styles and, subsequently, the verbal and visual narration (and styles) can be attributed to different persons. ${ }^{3}$ Next, I shall discuss the ways in which graphic style can be understood in comics. Subsequently, I will move onto the narrative uses of graphic style by way of stylistic variation and the so-called 'mind style'.

\section{Graphic Style}

In literary stylistics, the concept of style refers to patterns of linguistic choice and preference that can be attributed to a particular author's personal style, a period style, a generic style, or a given work of literature. ${ }^{4}$ In its broadest sense, literary style involves all possible linguistic choices in the text, whether lexical, grammatical, phonetic, or contextual choices, figures of speech, or any other. Subsequently, stylistic analysis focusses on such linguistic elements, patterns, and structures, provided that they are foregrounded in the text as having stylistic relevance. ${ }^{5}$ In film studies, David Bordwell has defined a film's style similarly as "a system of technical choices instantiated in the total form of the work, itself grasped in its relation to pertinent and proximate stylistic norms" $(2008,378)$. More broadly speaking, style in film is the use that an individual work of art makes of the medium: "the repeated and salient uses of film techniques characteristic of a single film, a filmmaker's work, or a national movement" $(1990,388)$. The stylistically important technical choices can, in Bordwell's model of functions of style, channel story information (denotative function), convey meanings (thematic function), 
signal a feelingful quality (expressive function), and exhibit perceptual qualities and patterns (decorative function) $(2008,377)$. All these functions of style are observable in comics as well. Our emphasis here, however, will lie on the channelling of story information through stylistic choices and variation.

In comics, graphic style has various potential functions: it marks the maker, a period, a genre, a particular work, or a contextual artistic reference; it connotes the cartoonist's tone, approach, and perception of the world and may create specific effects of realism, dream, memory, humour, suspense, and the like. In comics studies, Robert C. Harvey's definition of style as the mark of the maker and as the "visual result of an individual artist's use of the entire arsenal of graphic devices available, including the tools of the craft" $(1996,152)$, represents the traditional conception of the notion. The scope of this definition is relatively broad as it pertains to all possible devices and formal options available in the medium, from drawing techniques, the use of the brush and the pen, narrative breakdowns, and other compositional techniques, to layout style and the combined effect of all these devices, patterns of choices, and preferences. The scope of graphic style thus extends from the individuality of the graphic trace to the structural organisation of mise en page, that is, the broad functions of narrative organisation, selection, and arrangement of both words and images within the space of a page. However, the personal manner of holding the pen and the brush is central to this definition, and style is clearly understood as something that belongs to the artist, not the world that is depicted.

A narrower definition of graphic style can serve us to make it a more analytical concept, and more useful for the narratology of comics. And yet, the question of style should not be conceived too independently from the narrative function of channelling story information. For instance, Hannah Miodrag distinguishes between formal aspects of pictorial style and narrative functions of the composition. The former comprises pictorial elements in comics (and their semantic values) in their own right, comparable with traditional components in the art of painting, such as line and brushwork, light and shadow (chiaroscuro), texture, mass, order, proportion, balance, and pattern, as well as figures and composition (the ordering of the parts of the image into a whole) (2013, 198). By contrast, Miodrag includes in the narrative function of composition those forms of layout that "have no value in themselves" but only in relation to the narrative content that they organise $(2013,219)$. The problem here is not the analysis and appreciation of comics as significant works of art in their own right, but the distinction made between the pictorial elements of expressive line work, i.e. the visual form of comics on the one hand and their narrative content or strategy on the other. ${ }^{6}$ Beyond layout styles, graphic line and brushwork can also serve narrative functions, for instance, by helping to link panels in a sequence by 


\section{Graphic Showing and Style}

purely graphic means, much in the same way shots in narrative films are connected by a graphic cut. Visual style can also contribute significantly to the reader's understanding of a character or a storyworld. Think of the changing styles in Bill Watterson's Calvin and Hobbes (1985-1995) that generate from Calvin's fantasies, dreams, games, and perceptions (modality-to-modality transition). Such stylistic shifts and transformations, for instance, into cubism, the shadowy world of a hard-boiled detective or, most often, into the reality of Calvin's toys and games, from spaceships and dinosaurs to his favourite tiger, are indicative of the character's powerful imagination and capacity to immerse himself in the world of make-believe. And just consider how important graphic style is in constructing a fantastic world in Winsor McCay's Little Nemo (1905-1914, 1924-1927) or Fred's Philémon (1965-1986). In these two classic fantasy comics, stylistic features such as vibrant colours and the changing panel shapes stress the dreamlike inconsistency of Slumberland and the unreality of the letter islands of the Atlantic Ocean. McCay's and Fred's stylistic innovation and exploration of the spatial possibilities of the medium are inseparable from the worlds and the characters that their series depict. It is quite possible to examine their artwork in terms of formalist art analysis, but from our narratological viewpoint, we need to focus on the dynamic relationship between style and narrative meaning rather than seek to separate style from narrative content. The point in this is not to subject the issue of style to narrative sense-making, but to be better able to account for the narrative functions of style in comics.

In French-language comics theory, graphic style is frequently defined more narrowly as an instance of graphic showing, a personal graphic expression, or as "individual graphic writing" (écriture graphique singulière) (Marion 1993, 251; Groensteen 2011, 92). Graphic style, in this context, is a function of the graphic quality and identity of comics, but it does not extend to the broad functions of narrative organisation, selection, and arrangement of words and images-functions that these theorists usually relegate to an implicit and higher level "mega-narrator" (Marion) or "fundamental narrator" (Groensteen). ${ }^{7}$ Style, then, is conceived as a broad phenomenon and graphic style as one of its dimensions, a matter of pictorial choices that are stylistically relevant. Such relevance can be measured, generally speaking, by the prominence of certain devices and techniques of drawing (out of the arsenal that is available in the medium), their distinguishable (or foregrounded) qualities, and combined effects. One possible advantage of the narrower focus on graphic style is that it allows us to perceive style as a question of enunciation: to what or whom do we attribute graphic style? This is not simply a pragmatic question of attributing particular graphic features to a particular author, cartoonist, colourist, or their cooperation, but it involves the complex issue of the relation between graphic style and meaning, for instance, the narrative functions of style in terms of the presentation 
of the characters' mental life. Another advantage of a limited focus on graphic style is that this enables us to highlight the narrative dimension of visual style in comics. Obviously, the difference between layout, breakdown, and graphic style is not always that clear. Stylistic choices concerning, for instance, the drawing of frames, the gutter and speech balloons affect the way layout and breakdown may be conceived, while pictorial style can also be an efficient means of perspective-building, attributing perceptual information or types of perception to a particular character and/or world. For our purposes, however, a distinction between graphic style and other stylistic features, such as layout style, can usefully be made.

The aspects that may be salient in terms of graphic style in a given work of comics need to be evaluated on a case-by-case basis. This may, in principle, concern any visual elements in comics. However, we may identify certain general graphic features and techniques that are traditional means of style, and particularly well-suited and readily available for narrative ends. They include, for instance, graphic lines and line work. Perhaps the most famous and recognisable style of drawing in comics, and in particular in the European context, ligne claire ('clear line'), has been named after the use of clear strong lines of uniform importance. The 'clear line' style curbs the expressive use of the line, an alternative stylistic variant in many other comics. ${ }^{8}$ Another important conventional dimension of graphic style is the surface of the images and the page. Hergé's Tintin albums do not allow us to see the effect of pen work and brush strokes, whereas much of Joann Sfar's work, such as Le Chat $d u$ rabbin (The Rabbi's Cat), creates a bold impression by the use of expressive lines and the impression of a more or less spontaneous and intensive pen work, that the panel images, the writing and the frames alike, are made by the artist's hand in a kind of organic whole. Additional variants of graphic style so salient in the ligne claire school are the result of stylistic constraints that reject some conventionally available stylistic choices and techniques: the downplaying of contrasts of light and dark (or shadow), and the avoidance of shading techniques, such as hatching and cross-hatching. The uniform application of strong, flat colours marks another seminal stylistic feature of ligne claire. Other important variants of graphic style, but perhaps less prominent with regard to ligne claire, include the effects of volume (shapes, figures, ink), the impression of depth (variety of flatness and depth), the use of visual symbols, and the style of writing and lettering.

Consider, for example, how the three basic colours-blue, yellow, and red-in Tommi Musturi's Walking with Samuel (2009) function as a fundamental element of the protagonist's world. Throughout this wordless narrative, the three colours of the flag of Romania are featured in a piece of cloth that the protagonist Samuel carries with him, while inventing a number of new uses for this object, such as a towel, flag, armband, 


\section{Graphic Showing and Style}

blanket, sail, cloth, and bag. The colours have the synaesthetic quality of representing various sensory impressions and perceptions such as liquid (water or alcoholic beverage), smell (flatulence), sound (bird's song and Samuel's willow flute-playing emanate from the same colours), light (colours in a prism, fire, and the sun), and smoke (from Samuel's cigarette). In what is a potential reference to the cartoonist's stylistic choices, the colours are also seen as liquid for washing hands. Later, similar hands are shown in another double spread with a black background, to hold fire and point to the reader (Figure 4.1). Clearly situated outside Samuel's world, these hands suggest a great 'maker' figure, perhaps the artist's alter ego. All in all, the three basic colours simultaneously present the character's multisensory experience, the metamorphic quality of his world (all shapes change, but the basic colours remain), and the image-maker's stylistic choices in using expressive colours and colour schemes.

There is also a great stylistic range of options available in comics on a scale between diverse realist styles on the one hand and cartoonish styles and caricature on the other. This can be highly relevant, for example, in relation to characterisation and world-building. It is conventional to mix different degrees of realistic detail and caricature, pertaining, for instance, to the distinction between characters and their setting, or the foreground and the background of the image. Furthermore, the combination of different varieties of realism and caricature is a stylistic variant

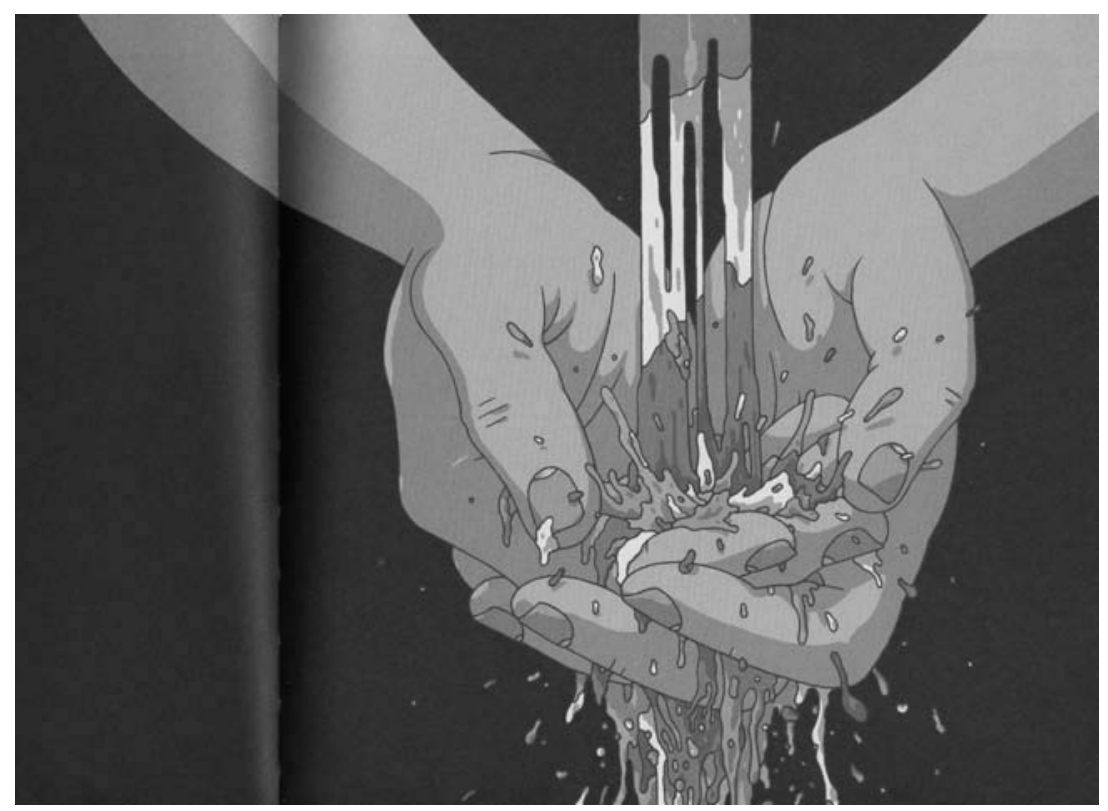

Figure 4.1 Tommi Musturi. Walking with Samuel (2009) @ Tommi Musturi. 
in its own right. Few cartoonists seek out-and-out photorealism. For instance, Tintin and ligne claire are characterised by a specific mixture of caricature and realism: the combination of cartoonish characters, and caricatured faces, with realistic and sometimes even photorealistic backgrounds and interiors. In particular, the main characters' faces are typelike, simplified, and condensed to a limited number of basic elements (eyes, eyebrows, mouth, and optional wrinkles) that can be easily manipulated to express recognisable forms of emotion and thought. There is no styleless comic in this respect. However, graphic style is a relative concept since the recognition of the salience of some stylistic variant in a particular work, such as the drawing of faces, requires comparison with the stylistic features in the rest of the work, or some other comparable body of work, or a particular tradition. The full stylistic effect of the graphic elements and techniques can only be measured in their combined effect with other stylistic choices, such as breakdown, layout, voice, the ratio of word and image, or perspective, and in relation to the narrative as a whole.

\section{The Narrative Functions of Stylistic Variation}

One particularly fruitful area for investigation in terms of graphic style is dynamic stylistic phenomena, such as stylistic heterogeneity, rupture, and shifts within a single work. From our narratological point of view, these stylistic features concern us for two reasons. First, stylistic variation makes stylistic choices and contrasts particularly conspicuous. Second, stylistic shifts can serve and highlight certain narrative and thematic functions, such as indicate a change of modality, perspective, narrative situation, temporal frame, or narrative level (between frame and embedded narrative or between different storylines).

Literary stylistics has shown how variation in style, even just a word or a banal sentence, may gain impact from the context in which it is found (Leech and Short 2007, 44). In comics studies, several scholars, including Philippe Marion, Thierry Groensteen, and Gert Meesters, have discussed the ways in which stylistic variation can raise questions about the cartoonist's identity and the conception of the work as a unified whole. ${ }^{9}$ A typical effect resulting from the use of heterogeneous graphic styles in one narrative, as they argue, is that style is no longer conceived of as a simple mark of the maker. Hence, what may happen when an artist adopts multiple styles in one work, such as in the French cartoonist Winshluss's (pseudonym for Vincent Paronnaud) parodic adaptation of Carlo Collodi's Pinocchio (2008), is that graphic style becomes an issue in its own right as stylistic variation points to the way in which the characters and their world are graphically rendered. Another consequence of the stylistic shift is the stylistic patterning of the work, such as through contrast or parallelism, which may contribute to the 
narrative and thematic meanings of the work. Drawing then demands to be regarded as a subtle medium that offers an infinite variety of expressive possibilities (Groensteen 2011, 125).

Let us examine stylistic shift and contrast in Winshluss's Pinocchio in more detail. One major stylistic contrast in this graphic novel is that between Pinocchio's tale-about a seemingly mindless robot and an out-of-control war machine who ends up being adopted as a boy-and Jiminy Cockroach's (Jiminy Cafard's) story. The alternating tales, which focus on these two characters in their respective settings, are intertwined through space in that Jiminy Cockroach, who is an alcoholic aspiring writer and a parody of Collodi's Talking Cricket and Disney's Jiminy Cricket (Pinnochio's conscience in the 1940 film), nests in Pinnochio's empty head. Jiminy Cockroach has found there a room of his own, after having been dismissed from his work. Pinnochio's eyes function as windows and doors to his apartment.

The stylistic difference between the alternating stories is striking. Winshluss draws Jiminy Cockroach's tale in a markedly simpler, sketchier, and looser style in black-and-white strips, thus emphasising the impulsive and introspective characteristics of the main figure (Figure 4.2). By contrast, Pinocchio's story is multicoloured, often includes strong contrasts of light and dark, and also represents a considerable amount of internal stylistic variation. Pinocchio's story comprises, for instance, some painting-like splash images of varying levels of detail, wordless passages in an expressionist style, parodic sequences of Disney animation and children's comics, and panels drawn in the style of British war comics. In addition, the two storylines utilise different modes of narration, and narrative situation, to the extent that the Jiminy Cockroach storyline, unlike Pinocchio's story, features external narrator's discourse and, on a few occasions, also showcases a narrator figure in the images with a highly schematised face who addresses his words directly to the reader.

The shifts between these two stories are frequently motivated by narrative. For instance, when Jiminy messes with the cables in the robot Pinocchio's head, this causes a dramatic short circuit that changes the course of the main tale. The two tales are also sometimes connected by gaze images, shared events, or the technique of graphic cut. In another shift between the two stories, the last panel in a sequence of Jiminy's tale shows the cockroach looking through Pinocchio's eye into the countryside. We then see Pinocchio walking, in the following Pinocchio segment, in the same scene, about to approach the house of the seven evil dwarfs and their captive Snow White (Figure 4.3). Yet another shift involves a raven pecking at the hung robot's head in Pinocchio's tale, and the sound of this pecking then wakes up Jiminy's drunken friend in the subsequent Jiminy passage. A stylistically striking shift occurs when Pinocchio is thrown into a fiery furnace at a toy factory, and in the 


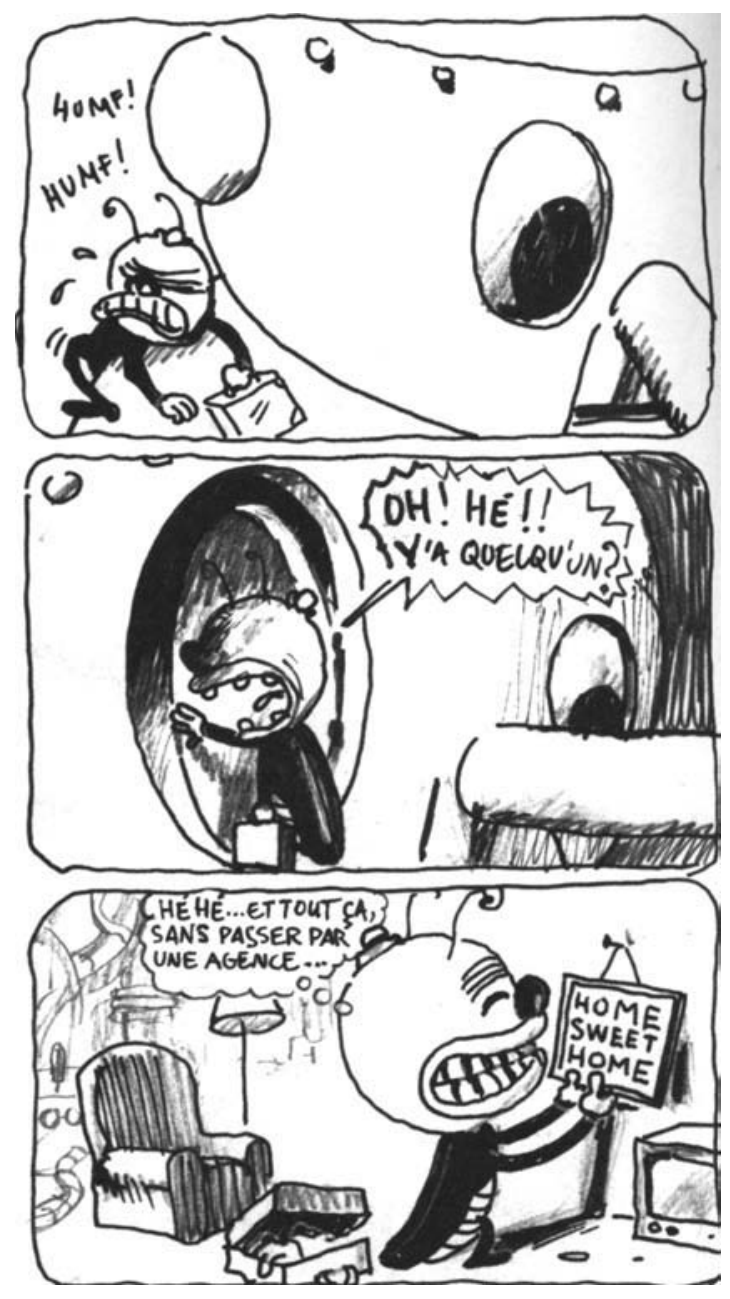

Figure 4.2 Winshluss. Pinocchio (2008) (C) Winshluss.

subsequent passage in Jiminy's tale, Jiminy feels like he is dying from the heat in his room. The drawings and the frames of this passage, "Jiminy Cafard in the heat of the night", are wavy and sketchy, thus reflecting the character's painful situation.

The stylistic shifts, therefore, accompany and amplify the transitions between the storylines and the focus on one character instead of another, and reflect the characters' perceptions and experiences. This is further evidenced in other shorter and wordless storylines that are interspersed with Pinnochio's and Jiminy's alternating narratives. These include Pinocchio's friend Candlewick's story ("Natural Born Loser"), told in flashback 


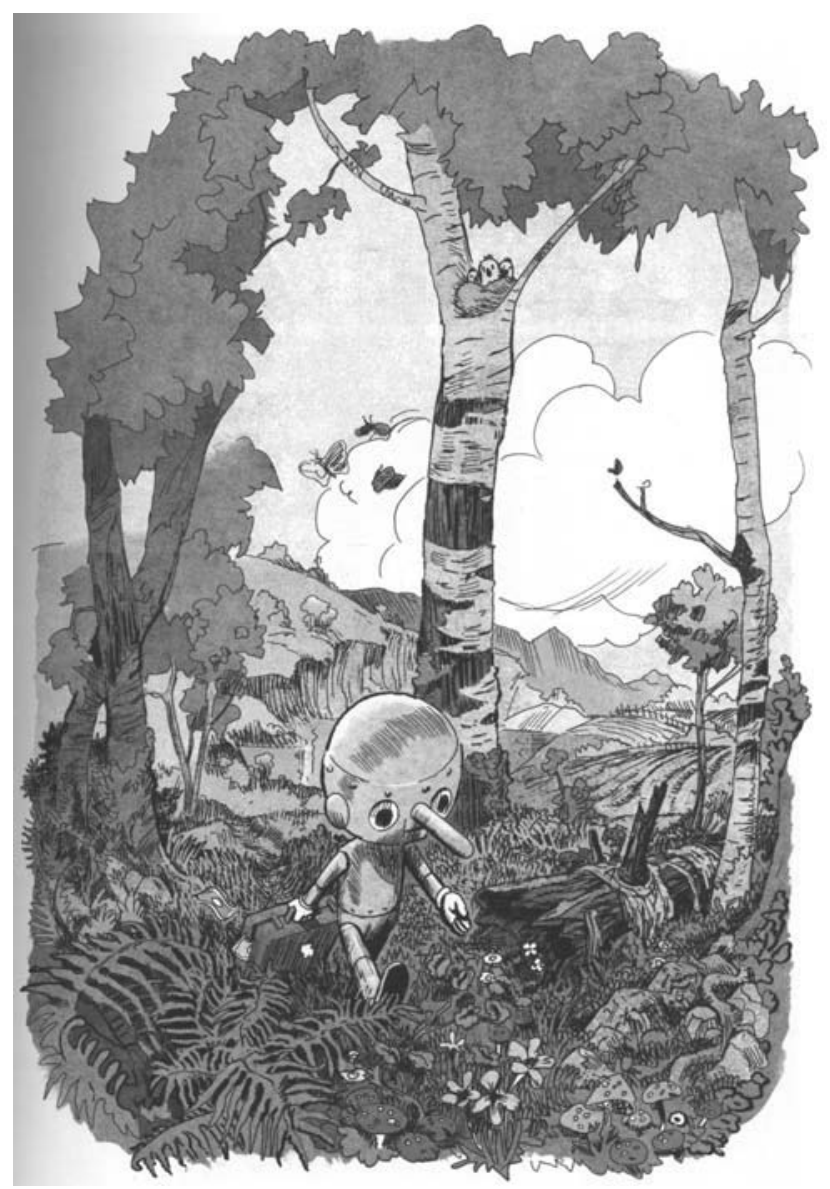

Figure 4.3 Winshluss. Pinocchio (2008) (C) Winshluss.

(for three and a half pages); the story, told in two instalments, about the farmer couple who lose their child at birth and who then adopt Pinocchio as their son; as well as a passage about the drowned Snow White and the surfer woman who rescues her. The stylistic features of these embedded stories are again quite distinct. Candlewick's and the farmer's tales have different monochromatic colour schemes, sepia-toned and hues of violet respectively, whereas the multicoloured four-page passage about the romantic encounter between Snow White and the surfer woman uses imagery that is reminiscent at once of romance comics and a racy $\mathrm{B}$ movie.

Graphic style is a highly dynamic feature in Winshluss's Pinocchio, but the result is not one of stylistic clash or unexpected discontinuity in the work. These shifts in style indicate changes not only in the storyline 
but also in the narrative focus and perspective, and they may reflect the character's experience, attitude, and emotion or the kind of world in which they live (dystopian nightmare, parodic Disney world, pastoral and nostalgic idyll, kitschy romance, and so on). Philippe Marion has argued that Fred's experiments with stylistic rupture can lead the reader to deny the mimetic "pseudo-evidence" $(1993,265)$ of the images, i.e. their reality effect and, therefore, perhaps better grasp the very consistency of their graphic traces. Given the dynamism of graphic style in Winshluss's parody, and the fantastic fairy tale world of Pinocchio, it remains unclear whether we can at all say that style establishes some reality effect. Perhaps rather, the stylistic variation in this work challenges the reader to think more deeply about the relation between style and narrative, style and perspective, or the meaning of style in creating a character and a sense of a world. Here, the varying styles reflect the narrative content, and contribute to it significantly, or perhaps even create it, at once undermining and reinforcing style as a marker of subjectivity.

\section{Mind Styles in Comics}

Yet another narrative function of graphic style is to dramatise a particular character's world view, perception, and habit of thought. In other words, a narrative can invite the reader to realise that certain choices of graphic style, such as stylistic rupture or variation, need to be attributed to an individual consciousness in the storyworld (rather than the author). Thus, the association between graphic style and a character's mind also has the potential to imply that the character's world view has profoundly affected the way in which the narrative is told and drawn. Thus, the impression that visual style or stylistic variation reflects the character's situation or state of mind is taken to another level, and in a way literalised, so that style per se seems to result from the character's mind and experience.

A useful way of thinking about the functions of graphic style in such cases is mind style. In literary stylistics, the notion of mind style is derived from Roger Fowler, who introduced the term to designate "any distinctive linguistic representation of an individual mental self” (1977, 103 ) in his stylistic analysis of prose fiction. More precisely, for Fowler, mind style is a realisation of a narrative perspective, and it is particularly detectable in clusters of linguistic features or techniques that give an impression of an author's or a character's world view. Geoffrey Leech and Mick Short elaborate on Fowler's notion, referring to it as the way in which prose style creates "a particular cognitive view of things" $(2007,28)$ that belongs either to a writer, a narrator, or a character. When a certain mind style can be attributed to a narrator or a character, this means that the writer slants the readers towards a particular narrator's or character's "mental set" (Leech and Short 2007, 151). The basic premise of mind style is that all systematic linguistic choices or patterns, 
such as lexical choices and patterns, figurative language, or conversational behaviour, may reflect style and, subsequently, the workings of individual minds in narratives.

The concept of mind style has roughly the same meaning as what will be later defined as cognitive focalisation, or what Alan Palmer calls "aspectuality". ${ }^{10}$ All of these notions-mind style, cognitive focalisation, and aspectuality-allow us to focus on certain textual and visual markers in comics as cues of a character's mental set or world view and, moreover, to interpret these markers in relation to an evolving frame of consciousness. In contradistinction to the other notions, however, the concept of mind style provides us with a focus on the stylistic dimensions of narrative. This is important in the sense that any systematic investigation of the presentation of minds in comics needs to incorporate the question of visual mediation in its diverse forms and must relate this question to the analysis of linguistic patterns-such as vocabulary, grammar, transitivity, speech representation, metaphor, conversational behaviour, and deictic choices (Semino 2011, 420)_given that the comic has words. Furthermore, the interaction between visual and verbal styles and their combined forms can function as a marker of a mind style in its own right. The study of mind styles in comics thus refers to those (fictional or authorial) minds to which we can attribute cognitive functions by way of linguistic, visual, and combined linguisticvisual patterns, techniques, and other stylistically important cues.

Let us think of some examples of character-bound stylistic choices in which style contributes to establishing a sense of a character's mental state. One remarkable aspect, for instance, about the stylistic heterogeneity in David Mazzucchelli's graphic novel Asterios Polyp (2009) is the function of graphic style as a means of characterisation. The various visual styles, such as expressionist, realist, or romantic style, abstract or mimetic style, and the changing colours and hues in the narrative correspond intimately to the characters' personalities and emotional states (and in some cases to specific events). Therefore, the various graphic styles and colours are metaphorically attributed to given characters, connoting their world view, experience, or emotional state. ${ }^{11}$ Furthermore, the association between graphic style and the characters' minds suggests that the individual minds have affected the way in which the narrative is visually told and organised. The situation clearly breaks with the conventional attribution of graphic style to an author and creates a unique form of multi-styled and multi-perspective visual narration.

Local stylistic changes or ruptures may also create an illusion of direct access to a character's psyche. For instance, Manu Larcenet's Blast (2009-2014), a four-part series about the homeless ex-writer and murder suspect Polza Mancini, includes various instances of local stylistic rupture in this sense. These ruptures, accompanied by the surprise effect of colour, illustrate a complex inner experience in the narrator-protagonist's mind. In these moments, which the narrator, Polza Mancini, calls "blasts", the black-and-white story incorporates children's colour drawings (the images 
are drawn by Larcenet's children, Lilie and Lenni). ${ }^{12}$ The drawings constitute a kind of colour explosion as if these colours were emanating from the narrator's mind. They first emerge around Polza's head and then spread all over the space of the image, sometimes superimposed on Polza's body. This suggests that the colour images are something that only Polza sees in his lonely moments. However, whether Polza literally sees these images remains ambiguous. While Polza, during these visions, occasionally seems to be looking at the drawings around him and even lifts his hands towards them, the images are also shown around his body and above him when he has his eyes closed (Figure 4.4). And, the drawings are associated with other figurations in his mind, such as a hallucination of the Moai statues, which frequently occur during these experiences. Later, it is revealed that the visions are stylistically related to the distorted figures in another character's, the schizophrenic sculptor Roland Oudinot's, obscene drawings and collages. The reader sees these too in colour panels (as if we were looking at Roland's notebook). While Polza's coloured visions are much more childishly drawn, the last part of the series also suggests a close affinity between these images in Polza's imagination.
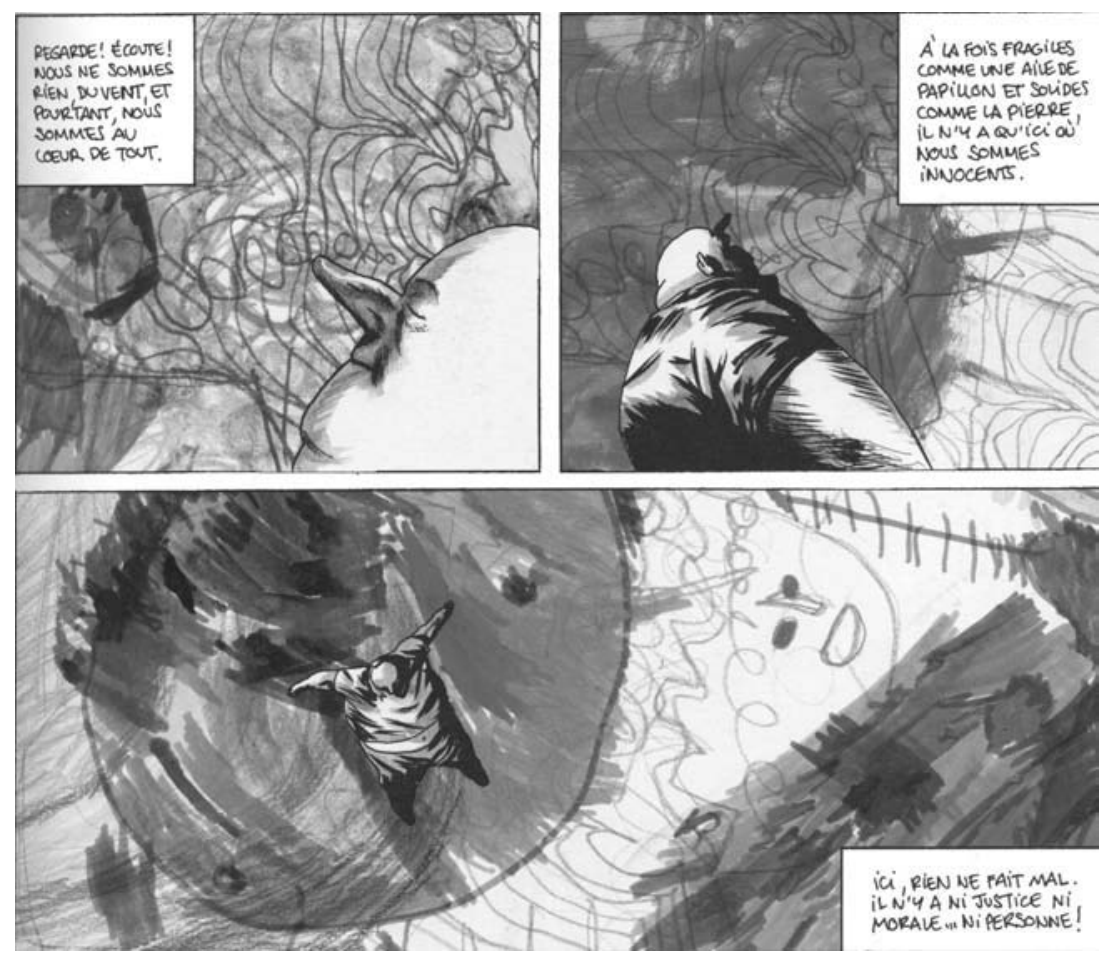

Figure 4.4 Manu Larcenet. Blast 4 (C) Blast-tome 4, Pourvu que les bouddhistes se trompent DARGAUD by Larcenet. All rights reserved www.dargaud.com. 


\section{2}

The stylistic contrast the colour drawings establish with the rest of the narrative dramatises the power of the experience, giving the readers a glimpse of the protagonist's inner perception and tumult. The vivid colours and the distinctly childish style of the drawings separate the blast sensations from his everyday experience. The narrator's verbal descriptions specify their meaning. Polza Mancini explains that the blasts involve sensations of fullness and of the instant, accompanied by a sudden clarity of vision as if the whole world appeared to him without morality or any preconception. The blasts are, as he specifies, an out-of-body experience of incredible lightness that allows him to hover above the ground, constituting a kind of rebirth or an 'intimate apocalypsis', one that implies a profound sense of union with nature. ${ }^{13}$

The verbal narrative track in Blast contributes significantly to a sense of a continuing-consciousness frame ${ }^{14}$ in these scenes, thus deepening the reader's understanding of the character's experience. Marion has pointed out that colour in comics can have an extensive impact on the development of an array of sensation, evoking a sensation of the real, while colour may also have a predominantly expressive and poetic function, presenting a high tenor of the graphic trace (1993, 156-57). The scenes of colour explosion and stylistic rupture in Larcenet's Blast series rely on both of these functions (referential and poetic) at once. Something similar happens in Marc-Antoine Mathieu's black-and-white graphic narrative $L a Q u \ldots$ (1991), in which the colour explosion at the end of the narrative marks the passage between the world of dreams and the world of reality. Both Larcenet's and Mathieu's works employ the sudden eruption of colour as a means by which to represent a character's inner experience. ${ }^{15}$ In Blast, however, this device is more systematic and accompanied by a more extensive stylistic rupture.

The concept of mind style provides us with an analytical frame for studying cases in which graphic style and an individual character's consciousness are intimately associated. Nevertheless, the limitations of this notion become apparent in wordless comics: precisely how should states of mind be ascribed to characters? ${ }^{16}$ For instance, Musturi's Walking with Samuel underscores the difficulty of drawing a coherent distinction between a character's consciousness and the fictional world since it is challenging, or perhaps impossible, to evaluate how much of what we see in the images is subjective. Beyond his muteness, the mystery of Samuel's cognition is further emphasised by the nearly expressionless face and lack of gestures. Due to sleeping, physical effort, or (perhaps) excitement over the course of the narrative, only some minute changes occur in his pupil-less eyes; the rare gestures he makes when he dances remain minimal (Figure 4.5). Furthermore, the storyworld, and sometimes the protagonist's body, is metamorphic and destabilised. Samuel seems at once to be able to live through the erosion of his world, while, at times, his body is manipulated and literally remoulded by huge hands 

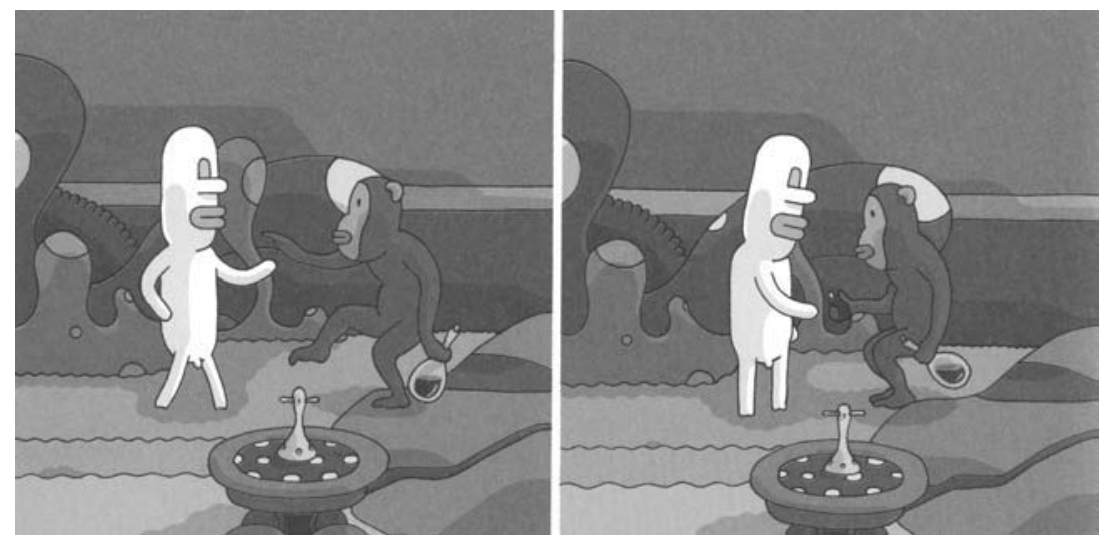

Figure 4.5 Tommi Musturi. Walking with Samuel (2009) (C) Tommi Musturi.

that suddenly appear. The six dictums that are listed at the end of the book, and that comprise the only words in the narrative (beyond the title), might represent Samuel's world view. Equally, they can constitute the author's interpretation of his character and the story. The dictums, such as "Do not be afraid", are followed by Samuel's gradual disappearance into the whiteness of the page.

\section{Conclusion}

The present chapter has focussed on medium-specific features of style in comics, involving specifically two questions of graphic style: stylistic variation and mind style, which pose important challenges for the analysis of subjectivity in this medium. The comics that have served as my main examples show us how graphic style can have many narrative functions by channelling story information. Graphic style is another means of connectivity and coherence between the images, and it helps to create a sense of a character and that character's experience, perception, and world. Stylistic variation and rupture may also be motivated through perspectival shifts or transitions between various storylines and different narrative levels. The above examples of stylistic variation, shifts, and rupture also suggest that the notion of mind style can be applied to comics where aspects of style reflect a narrator's or a character's world view. In other words, stylistic changes can be consistent with a given individual character's mind, emotional state, or mental state, and they may prompt us to imagine that elements of graphic style emanate from the character-narrator. I emphasise here the importance of imagination, and metaphoric attribution, and do not claim that the narrators of Asterios Polyp and Blast are or become agents who are responsible for stylistic 


\section{Graphic Showing and Style}

choices-graphic style is metaphorically associated with their consciousness and experience. What these examples suggest is a kind of thought experiment that invites the reader to question the distinction between the character-narrator and the agent responsible for stylistic choices. At the same time, it is important to realise that the application of the concept of mind style is open to challenge in a number of cases. The concept may lose its usefulness, for instance, when graphic style appears to remain so uncontrived that it is hard to distinguish it from a generic norm, when it is difficult to draw a coherent distinction between a character's consciousness and the fictional world. With regard to wordless comics, the challenge is to determine the degree of subjectivity of vision from images alone.

The functions of graphic style in comics are closely related to the general problems of perspective on the one hand and enunciation (source of narration), narrative agency, and narratorial idiom on the other. As we have seen, graphic style is a potential means of narration, perspectivetaking (from without and within the depicted world), and the creator's subjective expression. The importance of first-person narratives in contemporary comics and graphic novels is perhaps another factor that might inspire us to rethink the relation between narration, perspective, and graphic style. Yet again, since it may be difficult to agree on what exactly would be an 'implicit' narrator in this narrative medium, i.e. a narrator who is not a character and does not have a personal voice, the distinction between these categories in comics must be given some serious medium-specific attention. We will next turn our attention to the narrative situation and the issue of narrators in comics.

\section{Notes}

1 See Marion (1993, 249-253), Baetens (2001, 147), Bredehoft (2011, 109-114), and Gardner (2011, 54, 66).

2 Gaudreault and Jost (1990, 128-137) refer to “opérateurs de modalisation” ("modalisation operators"), such as flash images, which mark particular images out as mental images in cinema. See also Miller $(2007,106,119$, 122-123).

3 In Harvey Pekar's autobiographical comics, for instance, the question of the author's and the cartoonist's stylistic coherence and narrative control over the narrative is quite relevant. See Bredehoft (2011).

4 See, for instance, Leech and Short (2007, 10-11).

5 Foregrounding, a term used in formalist and empirical literary research, refers to the range of stylistic effects that occur in literature, whether at the level of phonology (e.g. alliteration, rhyme), grammar (e.g. inversion, ellipsis), or semantics (e.g. metaphor, irony) (Miall and Kuiken 1994, 390).

6 However, I find Miodrag's point that words and images, as different semiotic systems, maintain to a large extent their distinctiveness in comics despite their various forms and levels of collaboration, quite relevant $(2013,8-11)$.

7 One option is to equate all visual elements in comics, including page layout and framing, with graphic style. See Meesters (2010, 217). Jacques Lefèvre, 
by contrast, divides style in comics into the components of graphic style, the composition (mise en scène, framing), and the sequencing of panels $(2011,15,31)$.

8 The term was coined by the Dutch cartoonist Joost Swarte to refer to the school of work in the tradition of Hergé, to which Swarte himself belongs.

9 See Marion (1993, 262-267), Groensteen (2007, 98-100, 2011, 102, 124-129), and Meesters (2010, 232-233).

10 For Palmer, "whenever events occur in the storyworld, they are always experienced from within a certain vision" (2004, 51-52). See also Semino (2011, 418-420).

11 Fischer and Hatfield refer to Asterios Polyp by way of Palmer's notion of aspectuality to point out how conflicts between the protagonist, Asterios, and his wife, Hana, "are visually represented as non-compatible ways of seeing the world" $(2011,77)$.

12 Larcenet also uses colour in various passages in the series, where colour indicates a simple flashback or a recurring image in a dream. Another important stylistic rupture in the last part of the series is the coloured comic strip about a polar bear called Jasper (signed by "Milton Ferri"), apparently a children's comic that Polza reads with another character. The main figures of this comic also become the stuff of Polza's hallucinations.

13 Larcenet has described his fascination with children's drawings "without a code", comparing them to drawings by mentally disabled persons: "J'ai eu l'occasion de travailler dans un hôpital psychiatrique et d'observer les dessins des malades et c'est aussi passionnant que ceux des enfants. Ce sont des dessins qui n'ont pas de codes" (2011).

14 For Alan Palmer, continuing-consciousness frame is "the ability to take a reference to a character in the text and attach to it a presumed consciousness that exists continuously within the storyworld between the various, more or less intermittent references to that character" $(2010,10)$.

15 On how colour can function in a perception structure and character narration in films and how the origin of the colour may be metaphorically attributed to characters, see Branigan (1984, 94-95).

16 Groensteen poses a similar question about the interpretive challenges in wordless comics to anchor images to a subjectivity, including the difficulty in knowing whether what one sees in the images emanates from the reality or the imagination $(2011,137)$. 
This page intentionally left blank 


\section{Part III}

Narrative Transmission 
This page intentionally left blank 


\section{Narrative Agency (in Jiro Taniguchi's A Distant Neighborbood)}

The problems of narrative agency and enunciation have received much less attention in comics studies than they have in film studies. This is unfortunate because comics have important, medium-specific features in the way they tell stories. Many of these features we have already discussed, including the necessarily discontinuous form of the panel sequence and setup, and the expressive use of graphic style. Sequencing, perspective, layout, and graphic style can also draw the reader's attention to a kind of intelligence at work in visual narration, and these devices do this quite differently from, say, camera-movement in a film. Moreover, many signs that are typical of narrative discourse in comics, from the onomatopoeic Wham! or Pow!, to speech and thought balloons, cannot easily be placed within the word/image binary opposition since they are simultaneously visual and verbal, and are not analogous to the audiovisual means of cinema or theatre.

It is thus highly interesting, but also challenging, to describe how we go about constructing a sense of the prevailing frame of narration in comics storytelling. By this I mean a conceptualisation of a kind of global frame of narration that enables us to estimate the meaning and importance of the various visual and verbal or visual-verbal elements, their relations, and alternating perspectives of the story. The notion of 'narrative agency' is another name for such a global frame of narration, and it involves the question of the source of narrative discourse, that is, the conception of some agency or agent that is responsible for the selection, arranging, and distribution of the story material. ${ }^{1}$ The notion is not unambiguous, however, since narrative agency may be defined alternatively either as an extratextual source of the discourse, equalling a reader's construct of a kind of implied or inferred author who is prompted by the text, or an instrument or a structural principle within 'the text itself' that functions as a kind of reading instructions. Here, my intention is to combine these two views. Thus, by narrative agency, I refer to the ways in which a comic may acknowledge the source of narrative discourse. Ultimately, however, such acknowledgement depends on the reader's awareness and construction of that agency. 
It also needs to be asked to what extent our theoretical notions, such as narrative agency or a narrator, are informed by background assumptions about authorship and the practices of creative production in the medium. Presuppositions about authorial 'voice' or intention, authorship, and the process of production regularly play into the narrative analysis of comics whenever the analysis moves into the domain of interpretation. The question about the relation between narrative agency and the author's intention becomes relevant, for instance, when the reader tries to understand what someone intended to convey by writing or drawing a comic in the way that they did, and not simply to understand what the story means, what happens in it, or how the comic works structurally. Some of the challenges in this respect are medium-specific. As with films, comics are often collectively authored and produced-most mainstream mass-market comics and many independent 'auteur' comics are not created by a single author or consciousness-yet, unlike most films, a single author can also control the whole production. Thus, while many comics involve a complex set of relations among contributors, in many other cases we can refer to a single author. Moreover, joint authorship can take forms that are quite specific to the art form. This not only refers to the traditional division of labour in comic book or comic album productions, such as between the writer, the penciller, the inker, the colourist, and the letterer, but also the ways of conceiving authorship in the medium. ${ }^{2}$ For instance, some collectively produced comics are commonly identified with and recognised for the cartoonist's style (Jack Kirby), others for the writer's style and oeuvre (Alan Moore), and still others for the collaboration between a particular cartoonist and a writer (Jack Kirby and Stan Lee, Albert Uderzo and René Goscinny, Jean-Michel Charlier and Jean Giraud, and so on). Authorship is often collective in comics, but since joint authorship and production can take various medium-specific forms, the crucial question then is how individual and collective intentions may relate to each other. ${ }^{3}$ This is a particularly relevant question in many autobiographical and first-person fictional narratives.

\section{Theoretical Possibilities: Narrative Agents in Comics}

But how, then, do we conceive of narrative agency in comics storytelling? If narratological notions like 'narrator'-or other options that refer to the activities of a more or less personifiable narrative agent such as 'teller', 'presenter', or 'enunciator'-are problematic and potentially misleading, we are left with the difficult question of to whom to attribute the functions of selection, organisation, comment, and distribution. The problem is partly one of terminology, but it also begs the difficult questions of the specificity of the medium and the individuality of the narrative comic (versus what is common to all narratives irrespective 
of their medium). Let us first sketch out a palette of available theoretical options to address the question of narrative agency in comics, and then elaborate the question in an extended close reading of a first-person graphic novel.

\section{The Fundamental Narrator}

The available theoretical options, due to the composite nature of the medium of comics, are in many ways closer to the models proposed in film studies than they are to narratology that is based on literary examples, notwithstanding the essentially spatial nature of narrative organisation in comics and the lack of sound. One option is to hold on to the narrator concept and redefine it to fit it better to the medium. A strong version of this approach would be to argue that an implied or underlying narrator, or a narratorial consciousness, call it for instance a graphic narrator, is responsible for the whole narrative organisation, including the production of both the words and the drawings, as well as the showing of each panel image and scene. This position is one that Thierry Groensteen adopts in Comics and Narration, following Philippe Marion's earlier formulations (1993) and André Gaudreault's film narratology. It necessitates the distinction between the global and implicit 'graphic narrator' and any narrator who may be included in the storyworld.

Philippe Marion's narrative system of enunciation in comics is a hierarchical approach to narrative agency that gives the narrator concept a global role, as a kind of great graphic image-maker, and envisions new subcategories of enunciation that are specific to the medium. Based on Gaudreault's notion of a higher-level 'fundamental' narrator, a 'mega-narrator', or Great Image-Maker, who is responsible for both monstration (the activities of mise en scène and shooting) and narration (the editing of the images) (Gaudreault 1989, 88-89, 91-94), Marion's fundamental narrator is responsible for communicating the work in comics as a whole. Therefore, the narrator's activity in comics involves narrative breakdown and page layout, and is thus roughly equivalent to editing and montage in film composition, as distinct from the functions of the graphic sign and showing, e.g. presenting characters in action (Marion 1993, 193-194). Again in accordance with Gaudreault's model, the agent responsible for graphic showing is the monstrateur or monstrator (show-er). Graphiation, by contrast, is reflexive or 'autoreferential', directed to the graphic trace and gesture themselves (Marion 1993, 36). The two activities, monstration and graphiation, are partly overlapping due to the fact that, as Marion claims, the graphic trace in comics is always to some extent self-referential, i.e. marking the artist's style and subjectivity.

Groensteen derives his notions of the fundamental narrator (narrateur fondamental) and monstrator from these narratological premises. 


\section{2}

However, what is important from our perspective is that Groensteen sees the distinction between monstrator and graphiator as superfluous for the reason that the idea of graphiation basically indicates the 'haunting presence' of style in all drawn narratives rather than any separate level of enunciation (2011, 92-93). The question of graphiation can then be subjected to the issue of graphic style-a position that I also hold here. At the same time, however, Groensteen distinguishes between je montrant (a graphic shower who is responsible for showing the images), and the reciter, je récitant, the instance responsible for verbal enunciation (as in the narrative captions). Clearly, Marion's model neglects the issue of verbal narration and narrative instance, which can take both extradiegetic (narrator/narrative voice does not belong to the world of the story) or intradiegetic (narrator belongs to the world of the story as a character) forms. This is an important modifier to Marion's model: the issue of verbal narration needs to be given more attention, especially with regard to many of today's comics, such as Rutu Modan's Exit Wounds (2007), that create complex relations between the first-person voice and visual 'track' of narration. In Exit Wounds, the main narrative burden is seemingly carried by third-person narration, which focuses on dialogue and action, but it is interspersed at regular intervals with passages told by first-person narrative voice, a taxi driver called Koby Franco. These passages have the effect of subjectifying much of the rest of the story. Think also of the importance of the main character's statement of unreliability in Alan Moore and Brian Bolland's Batman: The Killing Joke (1988). The Joker is not a narrator, but his claim that he remembers his past sometimes in "one way, sometimes another", and further that "if I'm going to have a past, I prefer it to be multiple choice!", affects the reliability of the many subjective flashback passages in the story. The Joker can thus be perceived as a potentially unreliable focaliser.

At the same time, while more sustained verbal narration is crucial in many comics today, comics rarely show narrators in the images in their role of narrating. There are various significant exceptions, but these remain mainly local instances. One exception is Jack Cole's Betsy and Me comic strip series (1958), where the protagonist Chester B. Tibbit is shown as the narrator in the first panel of each strip, and Blutch's Blotch (1998-2000), where the character-narrator, the despicable commercial artist Blotch, is shown in the first panel of each instalment as a kind of master cartoonist. In Bryan Talbot's graphic narrative Alice in Sunderland: An Entertainment (2007), the cartoonist frequently depicts himself as the narrator who guides the tour of the history of Sunderland. In Marjane Satrapi's Persepolis (2000-2003), the autobiographical narrator-character may occasionally address the reader directly in the images where she is portrayed-thus the younger experiencing and the older narrating self temporarily fuse into one. The rarity and perhaps a sense of awkwardness in portraying the narrator in the act of narrating 
may be another indication of the importance of telling by showing. Relatively rare are those comics that would suggest that all images of the story are wholly marked by the narrator's subjective vision, as well as those, autobiographical comics exempted, that would imply that the images are somehow shown to us, or perhaps drawn, by the narrator-character.

A difficult problem that characterises both Marion and Groensteen's theories of graphic enunciation is the multiplication of narrative agents and levels, namely, the positing of various agents responsible for different functions, such as verbal narration and graphic showing and, further, the positing of hierarchical relations between these levels. In Groensteen's model, there are three levels of narrative enunciation at play. While any character-narrator, including the writer as the autobiographical character in their story, is subjected to the graphic shower in the images (monstrator), both of these agents, the reciter (or the character-narrator) and the monstrator, are subjected to and manipulated by the fundamental narrator (2011, 109-110). The fundamental narrator, furthermore, is also responsible for the panel arrangement and relations, and page layout whenever these elements have a narrative function. What confuses the hierarchical relations between these levels, however, is that on the level of graphic showing the monstrator is capable of acting to some extent independently of the fundamental narrator: "the narrator leaves to the monstrator the task of representing what the characters are up to" $(2013,96)$. The fundamental narrator, nevertheless, 'intervenes' in the monstrator's act of enunciation whenever that includes speech and thought. This is because, Groensteen argues, images are powerless to translate speech, and can only cite it in their space $(2011,106)$. Accordingly, in dialogue scenes speech would be represented as if the narrator had 'recorded' the characters' speech as it is supposed to have been pronounced and then reports that speech to the reader $(2011,106)$. Thus, one unfortunate consequence of this approach is that narrative analysis becomes increasingly interested in identifying the activities of various narrative instances and evaluating the relations between these modes, for instance whether one of the reciters or the monstrators is highlighted or backgrounded with respect to the other instance, instead of looking at narration as a whole.

The presupposition that dialogue between characters in comics is a kind of pretended speech act is problematic in at least two senses. First of all, this presumes that the characters' speech does not constitute a level of telling in its own right, but requires us to posit a fundamental narrator who channels and filters their narrative meaning to the reader. Certainly dialogue in comics is always mediated, for instance through layout, style of writing, or speech balloons, perspectival choices (point of view images), or the narrator's discourse that may filter and interpret the speech. Yet, the relevance in positing two different narrative instances and activities in conversational scenes, those of the monstrator and the 


\section{Narrative Transmission}

narrator, is not clear, and seems as superfluous as the distinction between the monstrator and the graphiateur. Second, the idea of pretended speech acts that are controlled by the fundamental narrator supposes that verbal narrative information in conversational scenes can be understood on its own, cut off from the way the scene is presented in the images. However, dialogue scenes in comics are typically based on the relation between represented speech, the speakers, and the other contents of the image. What is shown in the images affects the way in which the utterances can be understood, while represented speech guides us to look at the images in certain ways. Briefly, it is not necessary-and it can even be misleading - to posit two different agents of enunciation in order to analyse the narrative meaning of conversational scenes in comics. It may also be significant to relate speech in dialogue to narrative captions, or study and appreciate the effect of layout in such scenes.

The notion of the graphic narrator also begs the admittedly difficult question of how human-like or personal the graphic narrator may be conceived, in the sense of a human subject or intelligence communicating to his or her audience. Speaking of film narratives, Seymour Chatman has offered a clearly depersonalised version of the cinematic narrator concept, understood as 'the composite of a large and complex variety of communicating devices' $(1990,134)$. Other film theorists, however, have preferred more anthropomorphic models, amongst them the strong position that a narrating agency's consciousness and commentary informs each scene in a film (see Butte 2008). ${ }^{4}$ A further problem with concept of the graphic narrator is that it may be based on false assumptions about symmetric organisation between narratives in different media, for instance, that all narratives would make use of narrators.

\section{The Implied Author}

From the idea of some overall intelligence at work in comics, it is only a short step to the notion of an implied or inferred author, or some other author construct, that could be seen to be responsible for the choices and values of a given story. In fact, while emphasising the importance of distinguishing between author and narrator, Gaudreault perceived his 'fundamental narrator' also as an intratextual image of the real and concrete author $(1989,88-89)$. The advantage of the implied author theory is similar to the strong version of the graphic narrator concept in that it offers us a means by which to account for the possible discrepancies between the narrative as a whole and what is presented in words or what we may see in the images, including the role of character-narrators. In other words, the concept of the implied author is a theoretically grounded way-and a kind of compromise between text-centred interpretation and intentionalism-for talking about the constraints imposed on the understanding of narratives. These constraints are not created by 
textual (or text-image) elements alone, or directly related to the actual author's intentions and activities, but require that the reader makes inferences about that relation. The implied author approach also enables us to redefine the concept of the narrator in a weaker sense: the graphic narrator would then be responsible, for instance, for all narrative effects and conventions that are observable in a particular comic, or that can be inferred from the text, but distinct from any authorial element whose presence can be inferred in a work. Some of the uncertainties and problems embedded in the fundamental narrator and implied author approaches, however, are similar: is the implied author a textual and structural element or rather something that the reader constructs? ${ }^{5}$ How does one distinguish between those indexes in a narrative comic that indicate the activity of the narrator and those that may be associated with the implied author? Is the implied author an intratextual image of the actual author(s), or not at all an anthropomorphic figure? The use of any implied author notion requires a definition, and the definition in itself suggests a particular perspective on the question of enunciation. Subsequently, our choice is likely to influence the way the narrator can be conceived.

The two options of an extended mega-narrator or an implied author may or may not be accompanied by a (re)conceptualisation of the issue of narrative agency in comics through a new theoretical term. In film narratology, for instance, Manfred Jahn has coined the phrase 'filmic composition device' (or 'filmic composer'), which is in many ways comparable with Chatman's notion of an impersonal cinematic narrator, while Jahn refrains from using the concept of the narrator in the broad sense. This enables Jahn to give the concept of the narrator a strictly limited role, relegating it to an optional status, as in the cases of a voiceover or on-screen narrator. ${ }^{6}$ In comics storytelling, a similar 'graphic composition device' or 'comics composition device' could be envisioned. Concomitantly, a narrator in comics would then only refer to narrators as characters, or narrative voices when they can be distinguished from the author(s), i.e. cases when a narrative comic represents in some sense the act of narration itself, such as shows the narrator telling a story, while the overall narration is conceived in terms of an impersonal activity (narration) or 'device'.

\section{Impersonal Narration}

Finally, comics narratology can opt for not multiplying narrative agents, that is, not using any concepts referring to an agent, such as implicit narrator, implied author, presenter, enunciator, monstrateur, graphiateur, Great Image-Maker, or the like. In practice, this would mean defining narrative agency in comics as 'narration' or in terms of some other meaning-making activity, which can embrace the whole complex of 


\section{6}

narrative devices and expressive techniques in the medium without referring to an implicit agent of narration and enunciation. One advantage of this option is that it would allow us to keep better in view the question of reception and the readers' active production of meaning. One analogy in film studies for this position is David Bordwell's influential theory of film narration that rejects the idea of external personified agents such as the cinematic narrator to explain the organisation of a film. Bordwell's claim that in watching films "we are seldom aware of being told something by an entity resembling a human being" (1985, 61-62) seems a logical intuition, even if it is not based on actual empirical evidence concerning moviegoers' perceptions and sensitivities. ${ }^{7}$ Bordwell's theory of narrative agency in films does not adhere to a model of communication that would look at stories as messages from some sender (author, implied author, implicit narrator) to a receiver (reader, viewer, narratee), but stresses the role of narrative strategies, as means by which films may have a certain effect on the spectator. In this view, the viewer's meaning-making processes constitute the crucial constructive activity, and the issues of enunciation and authorial intention are to a large extent left aside. The concept of the narrator is not altogether abandoned in this approach, but it is relegated to those cases where there are evident traces of the presence of such an organising instance. ${ }^{8}$

It is useful, in order to avoid confusion between various narrators at different levels, to restrict the use of the narrator concept to the diegetic level. What matters, then, is our capacity as readers to evaluate changing degrees of subjectivity in visual showing and perspective, and to relate this information to changing degrees and types of verbal narration, and less to identify the presupposed overall authority of narration. Besides, there are two crucial general aspects about the reading and making of comics that need to be discussed and settled before adopting any strong notion of a graphic or fundamental narrator. One of them is that it may not always matter that much to the reader or viewer of comics who is responsible for the showing or organising of the images, or indeed if 'anyone' is showing or seeing at all. The authority behind particular choices in the images, or their perspective, may remain indeterminate without blocking our understanding of the story. This is not just because in fiction, in general, we can overlook inconsistency and paradoxical implications concerning the source of narrative information, ${ }^{9}$ but also that comics, fiction and nonfiction alike, enjoy considerable flexibility in alternating the subjective quality of the images on a scale from subjective to impersonal images. One option in comics, as in films, are images (or 'nobody's shots') that present reality "as if it were a question of seizing the essence of the action without underlining who runs through the shots or shows them" (Gaudreault and Jost 1999, 60). In comics, as in film, we may also accept, in order to believe in the narration, that despite the fact that the narrator himself is shown from outside in his story, the 
events and the world of the story may be presented in some sense from 'his' perspective (as he remembers them, for instance). In autobiographical comics, such as Persepolis, that are both written and drawn by the same author, the presumption of correspondence between the two visual perspectives-internal and external-may be particularly strong even if their distance may be equally important.

Still another point that should be made is that comics vary greatly in terms of the prominence of the verbal narrator, or narrative voice, within one work, as well as between genres and traditions. In the classical Franco-Belgian school of comics, for instance, the narrative is typically based on the mastery of the spatial organisation of the images and page, structured around dialogue and action, and the main character(s) movements from one panel to another. There is often an extradiegetic and heterodiegetic ${ }^{10}$ narrative voice in these stories, but this agent is transitory and highly restricted in its role to simple indications of temporal and spatial changes, or occasional short comments, for example, in Tintin, Asterix, and Lucky Luke. ${ }^{11}$ Narration through showing, breakdown, and page layout style, are essential to this tradition, but it is not clear why we should associate these activities with a separate agent. Moreover, in recent autobiographical comics, where the verbal firstperson narration is continuous, and the images are often subjectified in one way or another, thus creating a sense of a continuing-consciousness frame, it also seems counterintuitive to separate a graphic narrator, or a kind of comics mega-narrator, from the author as narrator who speaks in his or her own name. On the contrary, in fictional autobiographies, the distinction between the narrator and the author (or authors) is usually justified, and this is also the case with some autobiographies where the writer and the cartoonist are different individuals, but again, the choice of terminology should not be merely based on a wish to respect assumed symmetry in narrative structures across the media. If the theory does not aim to create new and better concepts, the choice of terminology should at least be flexible enough to accommodate genre- and medium-specific devices and their uses in this regard; narrative theory should, in fact, reflect such differences and not just infer and analyse similarities between different narrative media.

\section{The Story}

To illustrate and ground my general arguments about narrative agency in comics, I will draw on examples taken from the Japanese manga artist Jiro Taniguchi's 400-page, first-person graphic novel, Harukana Machi-e (1998), translated and adapted into French as Quartier lointain by Kaoru Sekizumi and Frédéric Boilet in 2006 and translated into English as A Distant Neighborhood by Kumar Sivasubramanian in 2009 (adaptation and layout by Sly Wind Tidings). Notice that I am not 


\section{Narrative Transmission}

only working with a translation of Taniguchi's novel but with an adaptation of his work where the writing, and the order of reading, and to some extent also the layout, ${ }^{12}$ have been changed, that is, westernised. Hopefully, the choice of the example does not limit the more general, theoretical nature of my arguments.

Taniguchi's autodiegetic narrative ${ }^{13}$ is split into two principal diegetic levels, a frame narrative and an embedded narrative. The frame narrative shows us the main character, a 48-year-old man called Hiroshi Nakahara, at Kyoto train station on the morning of April 9th, 1998. He is suffering from a headache after a night of heavy drinking. Intending to take a train back to Tokyo where he lives, Hiroshi, however, finds himself on a different line that takes him to his hometown of Kurayoshi. In Kurayoshi, Hiroshi visits his mother's grave where he kneels down to pray. The narrative then switches to a different time-frame where the narrator wakes up by the same grave on April 7th, 1963. In the ensuing tale, Hiroshi discovers that he is reliving his life as a 14-year-old boy, trying to prevent his father from leaving his family, while maintaining his adult consciousness. At the book's end the narrative shifts back to the frame narrative where Hiroshi, restored to his adult body, awakens by the same grave and returns to Tokyo.

An important feature of the narrative is the challenge that it poses to determining the reality of the embedded story, which comprises the major part of this graphic novel. The frame narrative lets us understand that Hiroshi is suffering from memory lapses, perhaps triggered by delirium or alcohol poisoning. Yet, the embedded narrative could also be Hiroshi's life-like dream, as implied by his waking up by his mother's grave. Furthermore, Hiroshi's prayer at the cemetery, and some of the symbolism surrounding this scene, associates his transformation with divine inspiration. Finally, various aspects of the story suggest that we are not dealing with a shift between ordinary and imaginary perception but with ontologically unstable worlds. The latter effect is created by way of the common fictional device of metalepsis, meaning an existential crossing or transgression of different narrative levels in a hierarchical structure (Genette 1980, 234-137; Fludernik 2003, 383). The most important proof of metaleptic infractions is that Hiroshi is pictured in the frame narrative receiving a novel from his childhood friend Daisuké whom he has re-met in the embedded story. Daisuké's novel has the same title as Taniguchi's A Distant Neighborhood, and the dedication in this novel, 'to the time traveller', is only understood if we take Daisuké and Hiroshi's meeting in the embedded tale for real (real from the perspective of the frame narrative, that is).

The following investigation comprises three narrative strategies that manipulate the relation between words and images, or verbal narration and graphic showing, and that can make us aware of narrative agency in this medium. These strategies include the use of subjective point of view (or the 
point-of-view image), the function of the spatially determined perspective in relation to alternating modes of verbal self-narration, and the role of wordless panels and sequences, seen from a non-character-bound viewpoint.

\section{The Subjective Point of View}

To return to my question concerning the global frame of narration, how does Taniguchi's first-person novel raise the question of narrative agency?

First of all, the subjective point-of-view panels of the story are carefully chosen instances that create meaningful contrasts with predominant impersonal or partially subjectified perspectives. The most striking instance of subjective visual focalising in the narrative is the moment when Hiroshi recognises his new (old) body as a young boy. In this passage, we see several panels as if through Hiroshi's eyes as he looks down at his hands, fingers, and the rest of his body, as well as his shadow cast on the ground (Figure 5.1). These subjective images create the mimetic illusion of direct access to the narrator-character's visual experience. In nonfiction, this would be equivalent to the witness's viewpoint that emphasises the immediacy of the experience. Here, in fiction, the focus on Hiroshi's hands, lower body, and shadow invites the viewer to share the protagonist's subjective viewpoint.

Notice, however, that the subjective point of view is not at all consistent in this passage, but alternates with impersonal perspectives that show different parts of Hiroshi's body from the outside. Furthermore, the position of these impersonal points of view is ambiguous in that, while they clearly show something that the narrator could not see, they also refrain from showing Hiroshi's face or eyes. Due to these effects of ambivalence in viewpoint, Gérard Genette's categories of internal, external, and zero-level focaliser become too rigid for our use (1980, 189-194). In most images included in the passage as a whole, the visual vantage point is clearly external but also limited by the character's field of vision. Simultaneously, on the verbal plane, we have full access to the character's thoughts and feelings (internal focalisation). The images clearly convey a sense of subjective perception, in varying degrees, but do so mostly-except in these few panels where we are looking with Hiroshi at his own body-without explicitly internal perspectives.

The effect of this alternating perspective is that the narrator's process of self-discovery is extended over several pages of the story, and the reader-viewer is prompted to participate in this discovery. We follow Hiroshi in the town's streets seen through his eyes, as it were. The stroll ends in a scene of self-recognition as Hiroshi sees a reflection in a window. Here, the subjective viewpoint gives way to a kind of graphic appropriation of shot-reverse shot technique in films. Now we see: (1) Hiroshi, seen from the side, noticing in a mirror a boy moving beside him; (2) Hiroshi, seen from the window looking at the window in awe; 

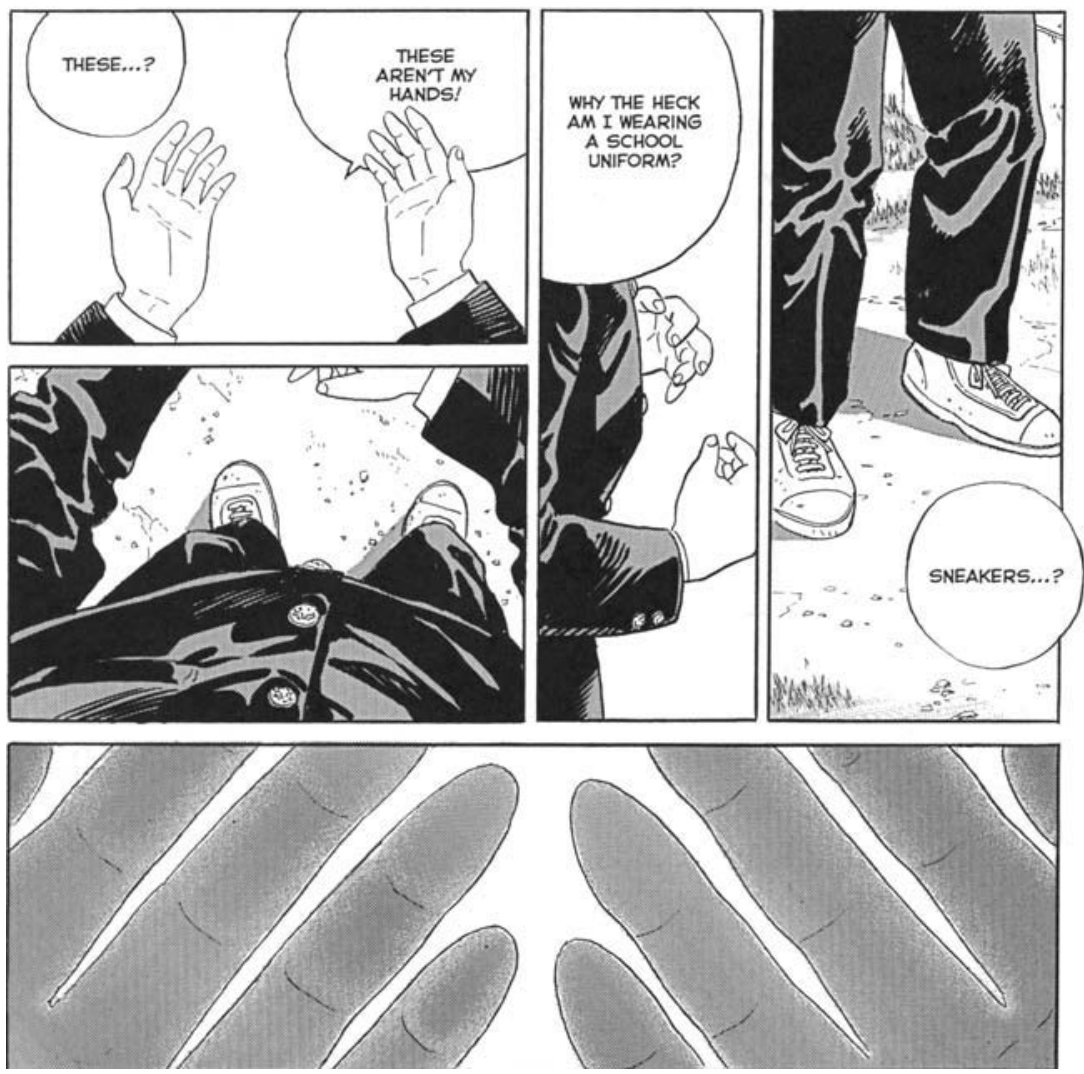

Figure 5.1 Taniguchi, Jiro. A Distant Neighborhood. Vol. 1. (2009) (C) 1998 Jiro TANIGUCHI (C) Fanfare/Ponent Mon, 2016 for the English edition. Www.ponentmon.com.

(3) Hiroshi, seen from his back recognising his face in the window; and (4) Hiroshi horrified at his image, seen from a perspective that is close to the gaze in the mirror image but not exactly the same (Figure 5.2). The final close-up lets us see his recognition of himself as a young boy.

We can make some general inferences from this scene. First of all, the subjective point-of-view panels typically remain localised manifestations. Comics where subjective focalisation extends beyond a few panels at a time are not common. If this were the case, the effect would be similar to a film where the camera eye could never leave a character's viewpoint and the person whom we follow and/or whose perceptions we share could never been seen from the outside except through reflections in mirrors and the like. Second, it is equally rare to find a narrative comic that would obliterate the authorial focalisation of the impersonal viewpoint throughout the story. In the best-known autobiographical graphic 

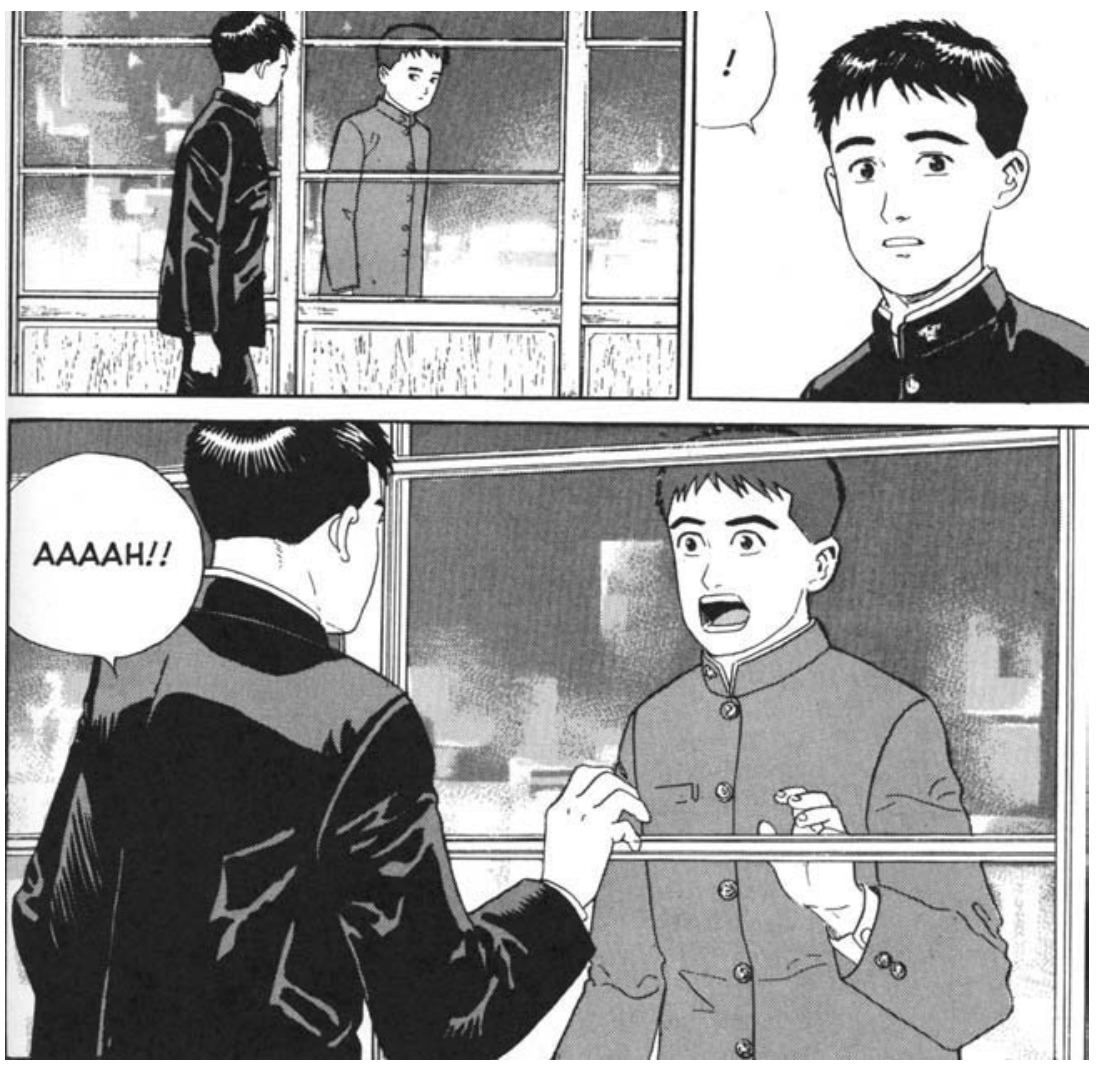

Figure 5.2 Taniguchi, Jiro. A Distant Neighborhood. Vol. 1. (2009) (C) 1998 Jiro TANIGUCHI @ Fanfare/Ponent Mon, 2016 for the English edition. Www.ponentmon.com.

narratives such as Keiji Nakazawa's Barefoot Gen (1973-1985), David B.'s L'Ascension du Haut Mal (Epileptic, 1996-2003), Marjane Satrapi's Persepolis, Alison Bechdel's Fun Home (2006), or in the works of Art Spiegelman and Will Eisner, as well as in much first-person fiction, the impersonal perspective remains predominant. At the same time, most of these works also include crucial, carefully chosen instances of subjective visual perspective. In contemporary documentary graphic narratives, as in Joe Sacco's reportages and Guy Delisle's travel stories, the witnessing subjective vantage point is widely used, but still the author is frequently shown from the outside, for instance, revealing his reaction to what he sees or situating him in particular surroundings and in relation to people around him. Autobiographical comics drawn by someone other than the writer, such as Emmanuel Guibert's Alan's War or Harvey Pekar's American Splendor, pose even more challenging questions about narrative agency in relation to 


\section{2}

the divergence of words and images. ${ }^{14}$ In such cases, it may be relevant to raise the question of the proliferation of sources of enunciation and the relation between the writer and the cartoonist as narrative agents.

Still another inference that we may make here is that comics can obfuscate the divide between external and internal focalisation on the visual plane, and that the verbal plane of narration can contribute to and even enhance such ambivalence. We can find another striking example of this strategy in the embedded narrative of A Distant Neighborhood, when the narrator sees and hears, in his drunken dream, a conversation between his wife and children at home. The unusual, furry-edged speech balloons, which are placed mostly on the frames of the panels, imply that the narrator is following the scene, is present in it as an observer, but cannot himself be seen or heard by the others. He is then disembodied in the scene in which he participates as an observer (Figure 5.3). ${ }^{15}$ As for the perspectives of the panels in this scene, it is possible to interpret all of them as belonging to his subjective viewpoint, but it is equally possible to maintain that only some of them approximate his supposed field of vision. The dream context, further, allows us to imagine paradoxical viewpoints. We may, for instance, suppose that in a dream the narrator could move anywhere without being seen, or occupy the camera-eye positioned above the scene, viewing as if from or through the ceiling. All in all, the narrator's gaze and his voice are paradoxically located both inside and outside the space of the images. Hiroshi occupies the position of a kind of frozen narrator who is spatially cut off from the world in which he thinks he is participating (at least as an observer). The effect of such partial or one-way metalepsis, which is restricted to the narrator's visual and auditory perception, would be difficult to achieve in literary narratives, where narrators who do not participate in the events, but who know the story world thoroughly, are commonplace.

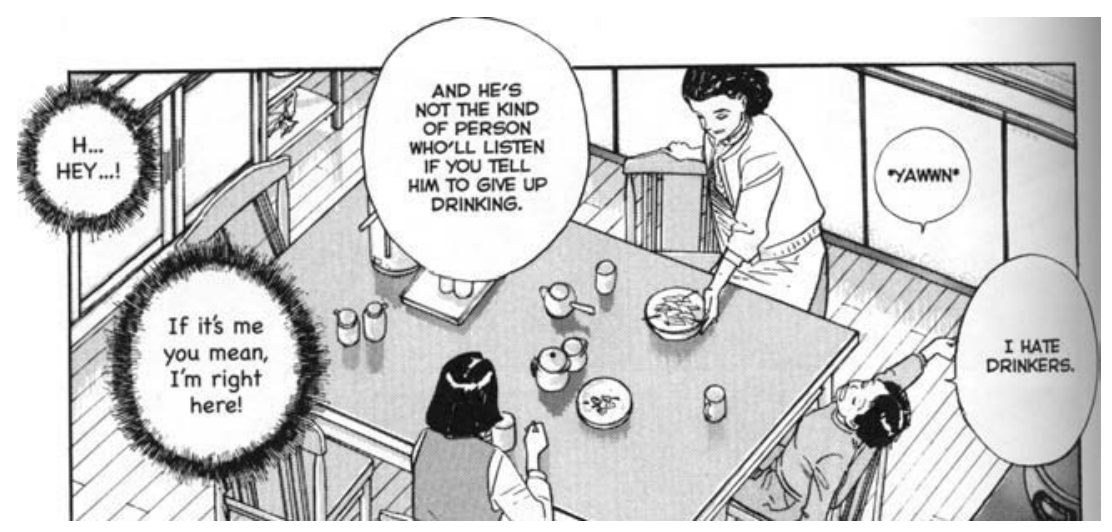

Figure 5.3 Taniguchi, Jiro. A Distant Neighborhood. Vol. 1. (2009) (C) 1998 Jiro TANIGUCHI @ Fanfare/Ponent Mon, 2016 for the English edition. www.ponentmon.com. 


\section{Alternating Modes of Self-Narration}

Another narrative strategy used in Taniguchi's graphic novel that is revelatory of narrative agency at work is the combination of intrinsic perspective of the images with two alternating types of verbal self-narration. A Distant Neighborhood, like many contemporary first-person graphic novels and narratives, employs a doubled verbal self-narration. This means that we have, on the one hand, the narrator's thoughts, including 'silent speech', as expressed in thought and speech balloons placed within the images. On the other hand, the story has a continuing narratorial voice, closer in many aspects to a written discourse, that is visually marked off the space of the image. For the latter kind of narration, Taniguchi uses fonts with a drop shadow and placed at the edge of the image frames.

Besides visual marking, the two types of self-narration are distinguishable by their varying temporal relation to the spaces and perspectives shown in the image. The speech and thought balloons represent the narrator's thoughts and speech in the present tense, the here and now of the moment that is shown in the image. The voice given in the drop-shadow text, which uses mainly the past tense, may include the narrator's thoughts at the same moment. ${ }^{16}$ For the most part, however, this voice serves the complex functions of thought report, including a summary of the narrator's ideas, expression of his emotions, memories, the meaning of his observations, descriptions of broader stretches of inner-time, and anticipation of things to come. In general, the second verbal narrative track is not only spatially distanced from the space of the image, but also allows more temporal freedom in the narration. At the same time, it gives the reader much more direct access to the narrator's subjective mental states. Swinging between internal and external verbal focalisation, the continuing narratorial voice of the past tense thus connects the two worlds of the adult man and the boy.

Occasionally, the voice in the past tense suggests that certain fields of vision may in fact belong to the narrator, meaning that something is perceived by him and not just focalised through the character in the images. One such occasion is the moment when Hiroshi sees his secondary school building and the narrator explains how the sight moved and almost thrilled him. Thus, the past tense in the narrator's voice maintains a certain distance from the experience, but the subjective perspective of the panel where the school is shown also closely ties the focaliser (i.e. the holder of the point of view) to the narrative voice. The fact that the panel is a point-of-view image is suggested by Hiroshi's look at the school in the previous frame. The narration in the thought balloons, in contrast, requires the narrator's physical presence in the image. The only exception to this rule-are instances when the narrator is shown looking at something and then we hear his thoughts in a balloon superimposed on what he is looking at. These images rely on the narrative context for being understood as subjective. An example of this is when Hiroshi verifies 


\section{4}

the date on which he is experiencing his new life from a newspaper. In other instances where the speaker of the balloon is not present in the image, as in the paradox of the spatially absent but verbally present narrator discussed above, or when we hear a voice from the television, the frames of the balloons are marked differently, such as with a serrated or jagged contour, to indicate the absent source of enunciation.

Verbal narration in the past tense, which is tied to the experiencing character's perspective, enjoys a significant role in the conversational scenes when Hiroshi Nakahara first meets his parents-his dead mother and his absent father-and other family members in the embedded story. Here, the narrative captions identify the milieu and the people he meets, and they also explain the narrator's emotions during the scene-he did not believe his eyes or was about to faint. This is a highly paradoxical situation: the narrator draws attention to his hindsight as an adult, whom he continues to be (inwardly) in the embedded story, but this hindsight-concerning what happened in the past and what consequences those events had-is not altogether helpful since he is reliving his past differently and perhaps in ways that may change his future. How would it, then, help narrative analysis to think that there is an extradiegetic narrator, who is responsible for the utterances of the dialogue, and possibly also for the perspective-taking and layout, while the character-narrator is in charge of the narrative captions and a monstrator does the showing by images? ${ }^{17}$ Instead of distinguishing between these narrative activities and their respective agents, and evaluating their hierarchies, it seems imperative to understand the relationships between these activities, and examine carefully the possible gaps between the diverse forms of narration and planes of enunciation.

All in all, the verbal strategies of split self-narration cue the reader to see the images in relation to certain temporal frames or changing temporal windows, marked by the narrator's perception and consciousness, even if clear cases of point-of-view images, belonging either to the experiencing character or the character as a narrator, remain singular instances. In order to generate a fully developed narratology of comics and narrative mediation in multimodal documents such as graphic novels, we must be able to account for such changing relations between various levels of verbal narration and visual showing, or between voice and the centre and focus of perception.

\section{Wordless Impersonal Perspectives}

Another means of manipulating the relation between the vantage point of the image and verbal narration in A Distant Neighborhood, and indication of narrative agency, is the strategic use of wordless panels with noncharacter-bound focalisation. These involve frames that can be attributed to narration, or the narrative event, and to that alone. The most striking examples of such panels, which remain localised instances in the story, can be found in the diegetic transitions between the frame and the embedded 
narrative. At these turning points in the narrative, marking the shifts between the narrative levels, we see scenes of the sky, the moon, or the cemetery from above. An important narrative function of this series of images is to emphasise maximum distance from the narrator-character's perception and consciousness. The changes in the frame size and shape, breaking the usual rectangular panel form into uneven frames or to full-page panels, further stress the sense of distance and transition. Several of these images portray a butterfly flying over the scene, and also approximate the butterfly's position in the air; sometimes the butterfly is also superimposed on the panel frames and the gutters to underscore the sense of transition between the spaces and temporal frames (Figure 5.4).

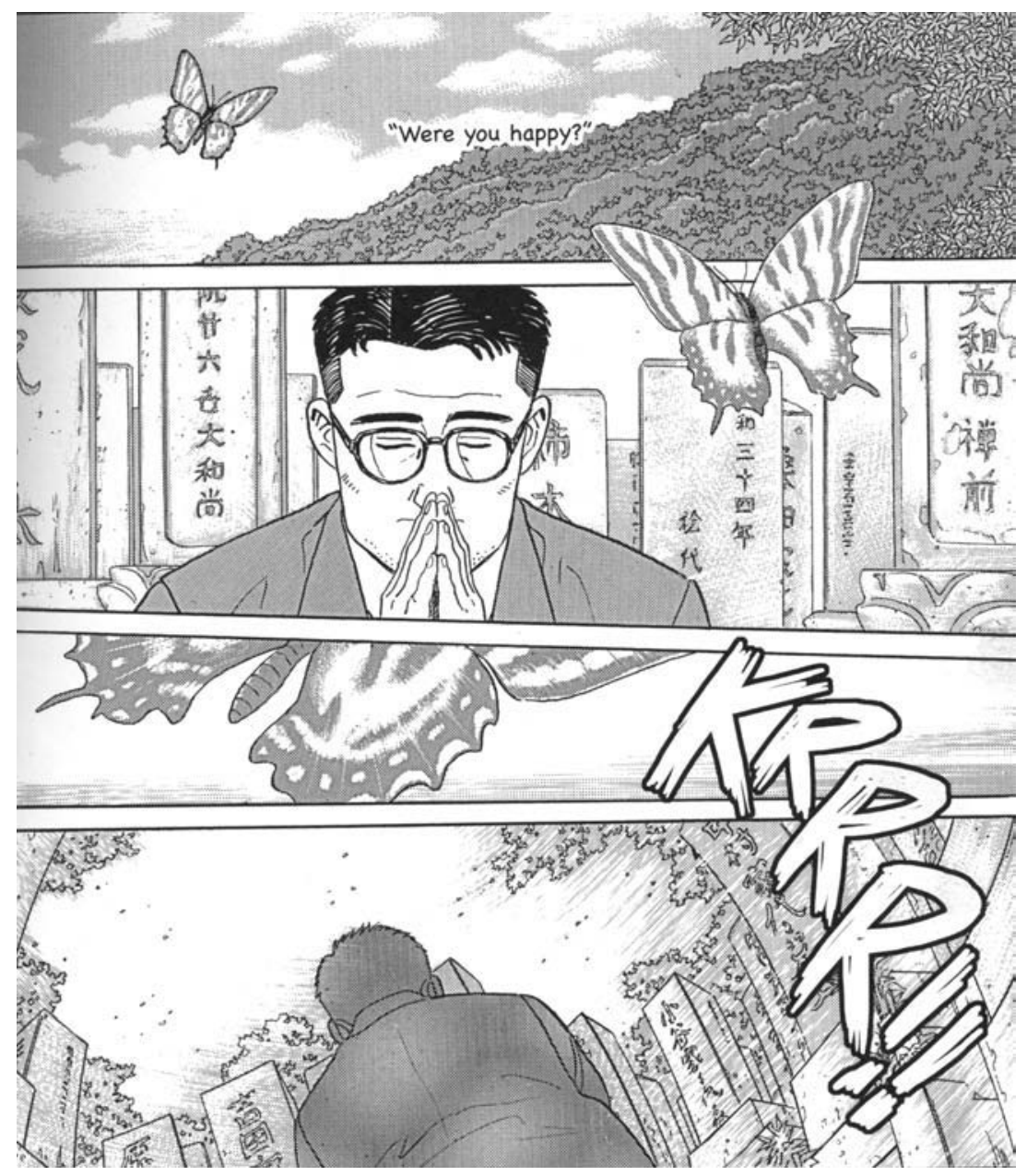

Figure 5.4 Taniguchi, Jiro. A Distant Neighborhood. Vol. 1. (2009) () 1998 Jiro TANIGUCHI @ Fanfare/Ponent Mon, 2016 for the English edition. www.ponentmon.com. 
Symbolically, the butterfly is a likely reference to the many Japanese stories of the butterfly as the wandering soul and as the soul of the dead. Many of these stories are of Chinese origin, ${ }^{18}$ including the Chinese philosopher Zhuangzi's famous butterfly dream: upon waking up Zhuangzi no longer knew if he was Zhuangzi who had dreamt about being a butterfly, or a butterfly dreaming he was Zhuangzi. Like this story, we can think that in the beginning and at the end of the narrative, Hiroshi Nakahara goes through a miraculous transformation in his dream, like Zhuangzi, and that he guides us to see the world from this perspective. Yet, how can we come to this conclusion if the point of view, after all, remains external and impersonal, and the butterfly is seen from the outside?

One way to answer this thorny question is to argue that the narrative perspective in the images of transformation supports the theme, namely, that the ambivalence of the perspective accentuates the double identity between the man and the butterfly. The scene builds on the formal convention, to which I have already referred, that comics can be clearly marked by a sense of subjectivity while the subject is shown from the outside, thus highlighting the question of the relation and distance between a perceiving entity and a narratorial identity. In comics storytelling, as in modern picture books, it is almost automatic to combine first-person verbal narration with third-person images, i.e. images that show the narrator from the outside. ${ }^{19}$ There is thus a potential disparity between what is told and what is shown. Hiroshi, as we learn in the course of the narrative, is uncertain about how to evaluate the reality of the embedded narrative, to know whether he is inside or outside of the story in which he participates. The lack of words in this sequence accentuates the uncertainty concerning the identity of the butterfly in the scene of transition, or perhaps, suggests that Hiroshi and the butterfly have merged into one. At the same time, other visual and verbal elements of the story accentuate the radical and deeply estranging nature of the transformation: in the embedded story, Hiroshi continues to think and speak like an adult resident of Tokyo, not like a boy in a small village in the countryside.

The function of the transitional panels differs fundamentally from other wordless panels in the story, where non-character-bound focalisation establishes scenes, focusing on the central themes and figures of each chapter. Some of these wordless images located at the beginning of chapters in fact imitate a photographic image, thus emphasising the visual or picture function (vs. narrative function) of a single panel image. Here the images, furthermore, seem to point self-consciously towards an authority that is capable of such combination and organisation, freely moving between various positions of telling and showing and occupying many points in time and space at once. Yet, even the impersonal field of vision of these images may sometimes encompass a personal one, for instance, by showing the main character's shadow in the foreground of the image. 


\section{Impersonal Visual Imagining}

Some recent theories in film studies have argued that the default mode of the spectator's engagement with cinema is impersonal visual imagining, but that there can always be suitably cued episodes of make-believe seeing from a subjective viewpoint (see Gaut 2004, 246). The features of perspective-taking in Taniguchi's graphic novel point in the same direction: at the visual level of the story, the impersonal perspective is the predominant mode of showing and the (purely) subjective point of view remains a localised instance of intervention. Impersonal points of view, however, despite revealing things that the character could not see, are often more or less related to, or encompass or are synchronised with, the character's point of view. They show fields of vision as if from the character's back or their side, illustrate the character looking at something, or include their point of view within a broader field of vision in another way. Purely non-character-bound perspectives remain localised instances.

The manipulation of the changing relations between the visual and the verbal register in this story also points to the conclusion that narrative agency in comics storytelling is not only a composite of different semiotic sources or channels, but is necessarily variable and morphic in its use of these sources. For instance, a continuing use of thought report is a strong indicator of first-person narrative. Yet, the verbal narrator's relation to the vantage point of the image-his presence as the filter and 'source' of the images that are shown — can best be evaluated by paying heed to the changing relations, including contrasts and incongruities, between words and images. The compositional techniques of the panel, the frame and the page setup, the balloon shape, or the onomatopoetic words and signs can also bear traces of perceptual activity, of mental and emotional processes of selecting, organising, and commenting on the images. Furthermore, various components of graphic style, for instance, a recognisable graphic line and trace, colour, or stylistic rupture, can be attributed to a certain source of enunciation and narrative discourse, and ultimately the author. The sense of mental processing implicit in such devices may sometimes be associated with the character-narrator, even if in general it remains impersonal.

Further, to study the narrative functions of the intrinsic (literal) viewpoint of the image requires one to revisit the basic narratological distinctions between who sees and who narrates, as well as the difference between so-called internal, external, and zero-level focalisation that is important in classical narratology based on the study of literary narratives. Such distinctions are different in comics that both tell a story in words and images and show a world in images. What counts, then, are the varying relations between a narrative voice, the verbal focaliser (i.e. the one who perceives things in verbal narration), the centre of 
visual perception (or visual focaliser), and the centre of attention (the visual focalised). Furthermore, in comics storytelling, these relations may involve different strategies of congruence and deviation between the components. We may equally pose the question of the reader's disposition to appreciate the medium-specific play on the anthropogenic distance between a narrating and an experiencing self, between a self who speaks, a self who sees, and a self who is seen. A Distant Neighborhood foregrounds the question of narrative agency by complicating the relation between the narrator's voice, his physical presence in the image, and the often photorealistically rendered world that the images depict. The narrator's bodily transformation, furthermore, emphasises the necessary split in the act of perception, possible in any homodiegetic narrative comic, between the experiencing and the narrating self, and between the personal and the impersonal vantage point of the image.

\section{Notes}

1 See, for instance, Seymour Chatman (1990, 113-119). I also draw on Donald Larsson's formulation of narrative agency: "Is there intradiegetic or intratextual evidence of extratextual agency? In other words, is there evidence within the diegesis or the text (aside from title pages, prefaces, acknowledgments and so on) of how this text came to be? How, if at all, does the text acknowledge (or pretend to acknowledge) that agency and why?" (2000). Larsson's model, in contrast to Chatman's notion of the impersonal "organisational and sending agency" (1990, 127), emphasises the reader's role, asking thus "what awareness of 'acting,' 'speaking,' and 'creation' we bring to fictional texts", and further, and how texts "encourage, evade, suppress, or direct that awareness" (2000).

2 Surprisingly little has been written about the theory of intention, authorship theory, or the history of collective production in comics studies to date. Some exceptions are Ault (2004) and Uidhir (2014).

3 This question has often been posed in film studies. See, for instance, Sellors (2007, 268).

4 For an overview of this debate, see, for instance, Gaut (2004), Thomson-Jones (2007), Bordwell (2008, 121-133). Similarly, in literary narratology there has been some well-argued resistance to the notion of the narrator, especially in relation to third-person narration. See, for instance, Patron (2009, 135-147). However, many literary narratologists also hold that the tendency to attribute stylistic features to a hypothetical narrator persona may be a kind of default expectation. See, for instance, Fludernik $(2001,622)$.

5 For a thorough discussion of this paradox, see Kindt and Müller (2006).

6 The filmic composition device is a theoretical agency and device that, as Jahn specifies, "need not be associated with any concrete person or character, particularly neither the director nor a filmic narrator" (2003).

7 Bordwell also argues that "very few viewers would take, say, a bit of actors' business or a pattern of lighting as having its source in an intermediary, a cinematic narrator" $(2008,122-123)$.

8 Gaudreault and Jost (1999) point out, however, how Bordwell's abstract instance of 'narration' is occasionally granted clearly human attributes. For example, Bordwell argues that: "Furthermore, the pensive ending 
[to Antonioni's La Notte] acknowledges the narration as not simply powerful but humble: the narration knows that life is more complex than art can ever be" (1985, 209-10).

9 See Thomson-Jones $(2007,84)$, Walton $(1997,65)$.

10 Genette distinguishes between two basic types of narrative with regard to the narrator's relation to the story and its characters: "one with the narrator absent from the story he tells" (heterodiegetic), and the other with "the narrator present as a character in the story he tells" (bomodiegetic) (1980, 244-45).

11 This is, obviously, a sweeping generalisation. See, for instance, Groensteen's insightful analysis of the different modalities of the narrator's intervention in Franquin's Spirou and Fantasio album L'ombre du Z (1962) (2011, 96-98).

12 Various changes occur in the translations: the placement of the narrative texts, speech balloons, and onomatopoeia in the image field often differ, the number of balloons per panel may change, onomatopoeias are sometimes omitted, the manga convention of using colour pages in the beginning is not respected in the English translation, and so on.

13 In Gérard Genette's terminology, an autodiegetic narrative is a first-person narration in which the narrator is the story's protagonist (1980, 245), and thus the narrator's experience and actions are central to the story.

14 See Baetens (2008, 84-85).

15 In contradiction with Jan Baetens and Hugo Frey's claim that in graphic novels characters are never disembodied $(2015,185)$.

16 Since there is continuous interaction between these two levels of first-person verbal narration, I find it problematic, and potentially misleading, to suggest, as does Badman $(2010,97)$, that narrative discourse in the thought balloons is not part of narration but monstration, i.e. showing and narrating by images, and an indication of internal focalisation at work in the monstration only.

17 One open question about the category of the monstrator in Gaudreault's theory is also whether the monstrator can have more than just a narrative role, such as a descriptive or painterly function. See also Gunning 2009, xxii-xxiii.

18 See Hearn $(1904,96-110)$. I am aware that the symbolic meaning of the butterfly as the soul, or as the soul of the dead, can be found in other cultures and traditions. For instance, Psyche of the Greek myths is a butterfly-winged goddess. I would like to thank Associate Professor Mayako Murai for her helpful suggestions about the myth of the butterfly and her interest in the presentation, based on an earlier version of this chapter, which I gave at Kanagawa University, Yokohama, on 24 August 2010.

19 See Nodelman 1991, 4; Yannicopoulou 2010, 66-67; 73-75. 


\section{Focalisation in Comics}

Perspective is a key aspect of transmission and mediacy in narratives, regardless of the medium. The choice of the kind of narrator or narrative voice, for instance, brings with it a narrative perspective from which the events, the characters, and their world are presented. The reflecting character through whom the events, other characters, and their world are perceived is another crucial technique of perspective. Beyond this, stylistic and compositional choices pertaining to language and visual expression, which cannot be attributed to a narrator or character, may also be conceived in terms of authorial perspective. For instance, in much of Chris Ware's work, such as in Building Stories or in his graphic novel Jimmy Corrigan: The Smartest Kid on Earth (2000), the graphic qualities of the text, and more precisely, the careful attention to the placement, shape, and colour of the graphic words and letters, functions as a form of perspective-taking.

Perspective, which is usually referred to as focalisation, is one of the most debated and researched questions in narratology. In his ground-breaking treatise Discours du récit, Gérard Genette undermined the hierarchy of 'showing' and 'telling', much used in earlier literary studies, by claiming that 'showing' in verbal narrative discourse can be only a way of telling. Genette then introduced the new term 'focalisation' as a replacement for 'perspective' and 'point of view' and made the distinction between narrative mode and voice. In this distinction, focalisation answers the question Who is the character whose point of view orients the narrative perspective? whereas voice pertains to the question Who is the narrator? More simply, Genette argues, this distinction can be formulated with the questions Who sees? and Who speaks? $(1980,186)$. The distinction between focalisation and voice is significant for narrative analysis, in particular, as it is possible for one person to express the perceptions and experiences of another. This can happen in real life as well, but it is characteristic of narrative fiction where narrators relate characters' vision and perceptions within their own discourse. If the distinction between voice and mode is not made, we may then not be able to sufficiently comprehend the role of mediacy in narrative fiction. Furthermore, from this distinction, Genette derived the well-known triadic typology of 
the focal possibilities of internal, external, and zero-level focalisation, based on the degrees of access to characters' minds in a given narrative (Genette 1980, 187-189). Internal focalisation, whether fixed, variable, or multiple in kind, involves a perspective that is limited to some character's mind. External focalisation, in contrast, is spatially limited to the role of the witness, without direct access to characters' psychology. Finally, zero focalisation, which Genette sometimes also calls 'omniscient focalisation' or 'non-focalized narrative', gives the illusion that the narrative perspective is spatially unlimited.

The concept of 'focalisation' has changed a great deal since these formulations, and Genette's basic premises have been brought into question numerous times. For one thing, Genette himself later redefined the question of Who sees? as Where is the focus of perception? $(1988,64)$, thus moving the question of perspective from some character as a seer to the problem of the affective, perceptive, and conceptual centre orienting the narrative. However, no character needs to embody such a centre of perception. Mieke Bal's early critical comments on this theory have also been highly influential in that she threw out Genette's category of external focalisation altogether because it rests on a confusion between Who sees? and What is seen? The category is really based on clues about how something is seen from the outside without the mention of inside views-if, that is, focalisation is consistent throughout the story (Bal 1991, 83-84; 1997, 142, 146). Bal thus redefined focalisation as the relation between the vision of the agent who sees (i.e. focaliser) - which does not need to coincide with any character-and what is 'seen' or perceived (i.e. focalised) (Bal 1997, 142, 146). Thus, any analysis of focalisation in narratives should investigate the relationship between the agent that perceives and that which is seen. Moreover, we need to add, with regard to visual narratives, the importance of the relation between what the characters are presumed to be seeing and the image field as a whole. These two can coincide in point-of-view images, when we supposedly see what a character sees, overlap in other cases to a varying degree, or remain more or less distant.

The re-emphasis of focalisation as a relation between the focaliser, the focalised, and the image field is significant in the context of comics storytelling since the person or character who is the focus of attention always has the potential to import perceptual information and subjective vision into the image. The general point that this brings to view is that there is a potential focaliser in every focalised person in comics: images reveal looks, acts of looking, fields of vision, and so on. This potential is similar to that of literary narratives, but images in comics also literally reveal acts of perception from the outside, that is, from an external viewpoint. Furthermore, other images in the sequence may always modify, through juxtaposition, the sense of subjective perception in a particular panel. 


\section{Narrative Transmission}

This chapter will concentrate on a limited number of vital questions about focalisation in comics, some of them medium-independent but others more medium-specific, that need to be accounted for in order to achieve a more comprehensive understanding of narrative perspective in this medium. These include the problems of:

- the relationship between narrative voice, mode, and showing,

- epistemic access to the point of perception and what is perceived, concerning the spatially determined point of perception in graphic images,

- the distinction between perceptual and cognitive focalisation,

- embedded and simultaneous focalisation, i.e. the simultaneity of different visual focalisers inside and outside the image frame,

- the complex scale of intermediate (visual) focal points between internal and non-character-bound positions, or between personal and impersonal viewpoints.

These questions involve visual information about observation, such as the centre and object of perception, the relation between various visual focalisers, and the degree of deixis ${ }^{1}$ and subjectivity in the field of vision.

In addition, I must make one further note about the consciously limited scope of this chapter: in what follows, I will be operating with a notion of focalisation that is restricted to the information that the narrative conveys about the spatial and physical point of observation, the sensory range of that position, and the spatio-temporal position of the focalised, that thing which is perceived. This is simply to avoid making the term too broad to be useful. I admit that it may not be possible to make an absolute distinction between an analysis of the spatial point of observation and, for instance, assumptions about the potential meanings indicated in what is seen. Judgements about internality, externality, or omniscience in narrative perspective are frequently accompanied by presuppositions that concern the limits of the narrator's or the character's knowledge and experience, or the epistemic motivation in perception.

\section{Who Speaks? Who Sees? and What Is Shown?}

Recently, there has been a strong trend in so-called postclassical narratology to emphasise perception as a fundamental cognitive frame in understanding any narrative, while these new theories have again challenged earlier definitions, typologies, and uses of the focalisation concept. Perhaps most importantly, Monika Fludernik has called our attention to the idea that focalisation is largely an interpretive category, or at least "not exclusively a textual category" $(1996,345)$, i.e. a postulation that is determined by the processing of certain textual clues, which in literature are verbal clues, such as deictic and expressive markers. The person who 
'sees', as she claims, "is the reader, but à travers the linguistic medium, and not in terms of visual perception" (Fludernik 1996, 345). For this reason, Fludernik argues that narratology has assigned false rigour to the distinction between 'who speaks' and 'who sees'. Furthermore, Fludernik's subordination of perceptional parameters in a narrative-or the perceptual metaphor-to the question of the presentation of consciousness may help us to see how it does not always matter who speaks or sees in the narrative (1996, 345-346). What may be much more important is how the reader, or the viewer, gets optimal information about a character's consciousness: his or her motivations, thoughts, and perceptions.

In the multimodal narrative environment of comics, the classical narratological distinction into voice and mode (or focalisation) must be modified for at least three reasons. First, the question of Who sees? or Where is the focus of perception? is relevant on two different modal levels simultaneously, both in words and in images. Focalisation is expressed in words in Taniguchi's A Distant Neighbourhood, for instance, when the narrator relates his emotions and thoughts as he looks at his little sister who is sleeping next to him ("I notice the light breathing of my sister sleeping beside me. It feels very strange"; "I can't believe that I'm sitting here like this...looking at my elementary school-aged little sister", 63). At the same time, focalisation is realised visually in a series of perspectival shifts between the narrator's gaze images and the object of looking at different angles.

Second, while the question of Who speaks? is significant in relation to verbal narration in comics, that relationship must also be reconciled with the visual narration and information. In the multimodal context, the formal markers of perspectival filtering, therefore, are necessarily both verbal (metaphorical) and visual (literal) clues that require the reader to integrate perceptual information from different semiotic channels in a meaningful way, for instance, in terms of complementary functions or contrast. Thus, the question of cross-referencing, or interplay, between visual and verbal information conditions the distinction between focalisation and narration. Wordless comics make an important exception in this case, as the question of Who speaks? can become largely irrelevant, even if, insofar as a wordless comic has a title, the issue of how to relate images to words remains pertinent.

Third, as comics can also narrate without any words, we need to add to the questions Who speaks? and Where is the focus of perception? another set of questions of narrative mode and mediacy: What is shown? and How is something shown? These questions underscore the importance of narration by predominantly visual means. Here, we could also refer to a kind of universal 'grammar' of pictorial narration where visual showing is essential in narration, that is, to the importance of showing (versus telling about visual perception) across various visual narrative media, from picture books to films and from comics to photo essays. 
In comics, therefore, the processing of narrative information involves paying attention not just to the distinction between Who perceives? and Who narrates? but to the interplay between a narrative voice, a verbal focaliser, a centre of visual perception (the visual focaliser), a centre of attention (the visual focalized), and the image field seen in the picture frame. We have to take into consideration the multiple ways in which the textual element (by which I mean written and drawn language) and visual focalisation interpenetrate each other and thus allow a multiplication of perspectives by way of typography, page and panel setup, and other means. Comics present a specific challenge to transmedial narratology in this respect, as the medium requires the reader to integrate perceptual information from different semiotic channels in ways that are both similar to and different from other forms of visual or multimodal narration.

Fludernik's claim about the perception of reality in films, put forward in An Introduction to Narratology (2009), points to a fundamental difference between verbal and visual ways of encoding narrative perspective beyond particular narrative techniques that may or may not be employed across media. This involves the question of epistemic access to narrative mediation, including knowledge of both the point of perception and what is perceived. Recent debates over narrative agency in film narratives offer us additional concrete means by which to conceptualise such a fundamental difference between verbal and visual focalisation. The basic idea in this debate is that film images give us a view into a space from some more or less determined perspective and that films, in fact, cannot avoid doing so.

Visual and multimodal narratives can employ a wide range of visual techniques as means of focalisation, including, for instance, techniques that may be associated with stylistic features in literary narratives but that do not have exact equivalents in literary discourse. In David Mazzucchelli's graphic novel Asterios Polyp, for example, every character is given an individual visual style, lettering, dominant colour scheme, and word/thought balloon format, and therefore all these techniques also become means of focalisation. Another important feature of perspective in Asterios Polyp, and one that would be difficult to realize in the moving images of a film, is the partial superimposition of panels on one another. For instance, the overlapping panel frames of a passage that relates the meeting of the main character Asterios and his girlfriend Hana with the composer Kalvin Kohoutek create a sense of a continuing space, while simultaneously they also divide the space into individual areas (the varying colours in the panels further sharpen that distinction). Thus, the 'polyphony' of these panels does not concern spatio-temporal transitions as may be the usual effect in the cinematic technique of the dissolve. Moreover, the overlapping panels may be taken as an illustration of the themes of simultaneity and polyphonic experience that are 
discussed by the characters in the panels. The split screen device in films may create similar effects of simultaneous events or disruptions of space, but the stress in Asterios Polyp is on spatial simultaneity and ambivalent spatial relations. There is no split screen. The speech bubbles enjoy spatial freedom from any screen structure, being both juxtaposed on the images and located outside the panel frames. The spatial organisation of the panel sequence and the page, as well as the distinct visual styles that are attributed to individual characters, highlight the importance of the questions What is shown? and How is something shown? in the space of the image.

\section{Spatially Determined Forms of Perception in Comics}

Narrative theory has to come to terms with the simple fact that images in comics, as in films, have an intrinsic, explicit point of view from which they are seen, no matter how personal or impersonal that perspective may be. In literary narratives, the sense of a physical, spatial vantage point from which the story is told may, likewise, be indispensable, serving as a cognitive frame in the understanding of the story (see Bortolussi and Dixon 2003, 171). Spatial positioning in literature, however, is crucially different from perspective in comics, in that prose fiction may relatively freely fuse, in any one sentence, the vision through which the elements are presented and the narrator's voice that verbalises that vision. ${ }^{2}$ Moreover, spatial positioning in literature is not an inbuilt feature of the medium. Genette has famously stated in Narrative Discourse Revisited that, unlike the movie director, "the novelist is not compelled to put his camera somewhere, he has no camera" $(1988,73) .^{3}$ As the invention of concepts such as Manfred Jahn's "zero", "weak", and "ambient focalisation" (1999, 98), and Fludernik's "neutral narrative situation" (1996, 172-177) also indicate, it is not always possible to locate any exact point of perception in literary narrative discourse. Point of view can certainly remain ambivalent in visual storytelling as well, but any image that creates the effect of three-dimensionality presupposes a perspective.

In literary narratives, further, we conceive propositions about focalisation and voice by determining certain verbal clues, such as deictic and expressive markers (see Fludernik 2001, 633; Herman 2002, 306-309). The sense of vision or voice in literature, therefore, is always a matter of verbal interpretation of another verbal representation. In comics, the reader may conceive propositions about the presented world both in terms of verbal clues (or metaphors of visual perception) and graphically rendered (literal) visual perception. When reading comics, we often literally see a person or a character in action, such as looking at something, from an external focalised perspective, or through the perspective that belongs to that person or is close to a figure who participates in the scene. The attribution of traces of personal or impersonal 


\section{Narrative Transmission}

intelligence to a continuum of a single individual consciousness in the images can be challenging. Still, this ambiguity has a different character from literary narratives.

When film theorists compare techniques of focalisation in film and literature, they often point out a crucial difference between these media in regard to the epistemic access available into the world that is seen. For instance, Katherine Thomson-Jones has argued that the "placement of the camera in filmmaking makes it impossible not to have an explicit point of view in cinematic narration, whereas literary events need not be described from anywhere in particular" (Thomson-Jones 2007, 88). Other film theorists, similarly, contrast the 'explicit focalisation' of films to metaphorical viewpoints in novels. ${ }^{4}$ The basic idea put forward in these approaches is that cinematic images give us a view into a space from some more or less determined perspective even if the degree of their deictic value or the degree of subjectivity of vision may be difficult to designate from the images alone. ${ }^{5}$ In comics storytelling, as in film, the point of view may always remain impersonal or ambiguous in terms of its possible subjectivity, but the image necessarily reveals a spatial point from which something (the focalised) is perceived. This constraint has various potential consequences for the understanding of the text-image interaction. One possible result is that it may not really matter that much to the reader of comics who is seeing, or if any person is seeing at all, since we know (culturally and intuitively) that images always show things rather than tell them, and also that if they represent three-dimensional space, they conventionally do this from a particular viewpoint.

There are some important exceptions to the general rule of the determined perspective, especially graphs and symbolic images, nonperspectival or multi-perspectival images, or polyphasic figurations that are used in comics. The non-perspectival images are 'pure' surface images that refuse the window-effect of a built-in perspective and the iconic expectation of 'pointing to something'. Such images, include, for instance, pictures that are blank panels or frames that include mere writing or speech and thought balloons, symbolic, abstract, or conceptual images, or scientific and technical ('objective') pictures that neutralise the viewer's spatial perspective. The latter category would comprise, for instance, maps, diagrams, charts, and geometric shapes. The case of the polyphasic image, where one picture juxtaposes or superimposes different points of movement or time, creating a more or less blurred image (Kolp 1992, 134-137), does not challenge the rule since it depends on the norm from which it is a deviation. Typically, in cases of effet Marey, ${ }^{6}$ the suggested ocular position, as well as the image frame, remains relatively stable, whereas the focus in the image undergoes changes in time, reflecting various stages of a process. By showing that the focus of perception is more or less undetermined, such images accentuate, as in the fight scene between Tintin and Doctor Müller in L'ile noire (The Black 
Island), a particularly hectic temporality. Similarly, a layered page or a double spread, which creates an effect of an ambiguous relationship between the frames and their backdrop, can manipulate the default mode of viewing from a determined point in space. Typically, the technique prompts the viewer to imagine different interrelated viewpoints. This is what happens, for instance, with what Groensteen has called a multicouche in shōjo manga (2011, 66-67), a multi-layered page layout typical of this Japanese genre that is especially targeted to girls and women. The same principle applies to split panels that juxtapose different points of perception and fields of vision.

While such exceptions are easily incorporated into comics storytelling, they usually tend to have limited and strategic functions, i.e. they remain localised instances. Many of them also depend on the general rule of the determined perspective. Typically, multi-perspective images, such as blurred or superimposed images, acquire meaning as perspectival distortions in relation to the expectation of a fixed viewpoint. Moreover, their visual form and frame may carry information about a particular (experiential, conceptual, stylistic, or other) viewpoint related to the other images around them.

\section{Focalisation and Ocularisation}

When a character in narrative fiction, regardless of the medium, perceives something, the object of that perception is regularly given and understood in the context of that character's thoughts, emotions, and experience. Thus, the distinction between perceptual and cognitive focalisation can be quite artificial. At the same time, the distinction between perceptual and cognitive focalisation, or perception and thinking (or knowing), is commonly made in film narratology, where it has been argued that in film narratives, the point of perception and the cognitive perspective (or attitude) do not have any obligatory correlation. The argument is not only medium-specific, but it also carries significance for other forms of visual storytelling. François Jost, drawing attention to how Genette's focalisation concept refers simultaneously to the act of perceiving and the acts of thinking and knowing (1983, 195; 1987, 21-22), has pointed out that this confusion becomes particularly problematic in the analysis of film narratives, where not all uses of the terms 'perspective' or 'focus of perception' are metaphorical. ${ }^{7}$

In films and comics, we need to come to terms with the fact that the image has a certain deictic value in itself (as pointing to something), and that the point of view is their inherent meaning-making mechanism. The representation of perception in visual narratives must also be conceived differently from those in literary narratives due to the functions of showing, including the showing of deictic relations in space, the literal spatial positioning of the focus of perception, and the spatial impact of 
the frame. In films, as Jost argues, perceptual and cognitive focalisation can have clearly separate functions, expressing quite different things $(2004,78)$. While in literature, we may need to distinguish between a narrator (who speaks) and a focaliser (who perceives), films may prompt us to further distinguish between perceptual (ocular position) and cognitive orientation. In films, Jost points out, "it is possible to show someone or something and at the same time express something completely different through the voice" $(2004,73)$. At the same time, it is possible for the perspective in films to be simultaneously 'inside' and 'outside' a character, i.e. to see a character from the outside while the perspective is also to some extent tied to the character's perception and experience.

Jost thus distinguishes perceptual focalisation (what he calls "ocularisation"), meaning the "relation between what the camera shows and what the characters are presumed to be seeing", from cognitive focalisation, or what he calls 'focalisation', designating "the cognitive point of view adopted by the narrative" $(2004,74)$. Similarly, despite the absence of a camera in comics, the manipulation of the relation between what the image shows (from some perspective) and what some character supposedly sees is an essential means through which comics can create a sense of a subjective vision. Not surprisingly, then, Jost's distinction has been increasingly applied with varying degrees of theoretical rigour and clarity to comics and graphic narratives. ${ }^{8}$ The same premises about perspective-taking in cinema apply to comics storytelling, with some obvious differences of technique, such as camera movement, or the means of aural perspective in films.

However, it is important to specify that Jost does not define focalisation (as distinct from ocularisation) purely in cognitive terms, but claims that the distinction enables us to focus on a relation between perception and knowledge. Focalisation, he argues, is "a complex product of what one sees, what the character is presumed to be seeing, what he or she is presumed to know, what he or she says, and so forth" $(2004,74)$. In other words, in Jost's definition, focalisation concerns the relation between the ocular position that the image postulates, the visual field in the image, and the character's speech, thoughts, and presumed knowledge, while ocularisation refers to the visual representation of perception in a literal sense. The analysis of focalisation thus addresses the way in which cinema works in several registers at once, combining the visual, aural, and verbal narrative tracks, to give the reader an illusion of an individual mind, while ocularisation characterizes visual information about the point and focus of perception only (and auricularisation describes the centre of auditory perception).

The ocular position is a prerequisite for the impression of depth in the image. Ocularisation, however, does not have to belong to any person or instance in the storyworld or in the frame narrative; it can remain fully impersonal and hypothetical. Thus, Jost's category of "zero 
ocularisation" does not refer to a missing point of perception, but to a broad range of perspectival options where the focalizer remains impersonal. Equally, the distinction between external and internal ocularisation is potentially misleading in comics because purely internal or purely external points of perception are usually only short instances. The crucial distinction, then, is not between external, internal, or zero-focal positions, but how and to what extent the image, and what is seen in the image, is subjectified by narrative conventions and context and associated with the perception and consciousness of a character in the storyworld.

Jost's categories of narrative perspective in films are useful in the analysis of visual focalisation in comics given that we do not make the distinction between perceptual and cognitive focalisation mechanistically. I see three main reasons for developing the dimension of perceptual focalisation in comics narratology. One reason for this is that the (narratological) functions of the point and focus of perception in comics, and the visual encoding of narrative perspective in this medium, are only partially understood at present. Therefore, we must try to take the question of the perceptual and, more precisely, optical dimension of focalisation in comics to its outer limit-to see what can be said about the specificity of comics in terms of narrative perspective-before a more holistic theory of perspective can truly be developed. Second, in a medium that is much less reliant on narrator figures than literary prose fiction-even in first-person graphic narratives-it is important to thoroughly investigate all relevant aspects of visual narrative mediation, including not just the point and focus of perception, but the functions of graphic showing and style, to better understand how minds and worlds are created in comics.

A third reason for focussing on ocularisation in its own right is that it allows us to recognize some of the formal options and narrative devices that are available in comics to present the characters' minds. The manipulation of the ocular position in comics can create various kinds of effects that may be important for the understanding and enjoyment of the story. For instance, this can contribute to an effect of ambiguous subjectivity and communal perceptual focalisation that would be difficult to imagine in literary narratives in quite the same way. In Matthieu Bonhomme and Lewis Trondheim's Omni-visibilis (2010), the whole storyworld shares the sensory perceptions of the main character-narrator Hervé, who is an office employee in his thirties. The point of perception is often the very basis of the parody of continuous webcam presence. In Omni-visibilis, all people, even from other parts of the world, begin to see through the protagonist's eyes when their eyes are closed, hear what he hears, and feel what he feels. Especially interesting in this light are the point-of-view images in the story. For instance, when the main character sees his pursuers through a peephole in the door, this cues the reader to imagine that the perspective belongs both to the protagonist 
and his pursuers. Furthermore, it is implied in these images that the people whom the readers see with the main character's eyes can also observe themselves from the same viewpoint. Thus, the experience of communal visual perception reaches new heights of ambiguity. The verbal narrative track is crucial to knowing what exactly is happening, but without the skilful manipulation of the ocular position, the whole point of the parody of webcam presence and surveillance, and paranoid reactions to webcams, might be lost.

In films, as Jost points out, verbal anchoring can easily transform internal into external ocularisation or vice versa $(1983,196)$. The same is true of comics. However, in a wordless comic such as Tommi Musturi's Walking with Samuel, shifts in perceptual focalisation can be detected by visual clues alone. In this story, the reader is suddenly invited to share a perspective that appears to belong to a bird that pecks the protagonist's eye. In order to understand the viewing position, the reader must compare this image with the surrounding sequence of images and the respective perspectives they offer. This wordless comic underscores the important role played by following image-to-image and scene-to-scene transitions in the representation of perspective, but, equally and efficiently, undermines the degree of deixis in the image, that is, the distinction between a character's consciousness and the rest of the fictional world.

I would like to conclude this section with the idea that Jost's ocularisation concept itself is not necessary in comics narratology, ${ }^{9}$ but that a systematic assessment of the distinction between perceptual and cognitive focalisation can improve our understanding of the multimodal dimensions of focalisation in comics and graphic novels. Such an investigation involves the study of the relation between the point of perception and what is seen in the image, the interaction between perceptual and cognitive focalisation in a sequence of images, and the relationship between visual and verbal focalisation. In other words, for the theory of narrative perspective to develop in comics studies, it is imperative that we investigate how visual perspectives are created and manipulated so that they become associated with a certain subjective vision or cognitive attitude. Equally, this requires that the criteria that help us determine how and to what extent something in the image is subjectified should be defined.

\section{Simultaneity of Different Focalisers Inside and Outside the Picture Frame}

All focalisation, be it in a literary or visual narrative, is typically variable. Whether in literature, films, or comics, perspectives vary between more or less subjective and objective viewpoints, or between different narrative levels, and can shift between different perceiving characters, or between narrators and characters. While proposing his original typologies and distinctions, Genette was fully aware that it is difficult to find 
pure examples for his three basic categories of focalisation: external, internal, or zero focalisation $(1988,73-74) .{ }^{10}$ Yet, in visual and multimodal narratives such as comics, the variety of forms of focalisation seems even greater. We need not only a revision of these categories, but the creation of more flexible ones.

As can be easily shown in film narratives, several internal and external focalisers can appear simultaneously at different points inside and outside the image frame. In his analysis of classical films, Celestino Deleyto has analysed the tendency to use external perspective to make the internal gaze understandable $(1996,223,226)$. Similar practices and effects are known to us from picture books, where it is a common convention to combine first-person verbal narrative with uninvolved third-person visual perspective.

While different internal and external focalisers can appear simultaneously at different points inside and outside the picture frame, this capacity can also set up tensions between the character's perception and the field of vision on the one hand, and between visual and verbal narration on the other. Playing with the divergence and convergence between visual and verbal perspectives is a common practice in autobiographical and other kinds of nonfiction graphic narratives. Typically, contemporary first-person graphic novels, such as Taniguchi's A Distant Neighbourhood, create a meaningful contrast between the narrative 'voiceover' and the narrator who is seen in the image from the outside. While the 'voiceover' narration often offers a clarifying perspective on the contents of the image, indicating what is important and salient in the things that are seen, the words may also maintain an ambivalent relation to the space of the image. This is different from literary narratives, in which the ability to present characters at the same time from the outside and from within is usually another indicator of the fictionality of the world, and the notion of simultaneity in this respect is more metaphorical.

To illustrate these points about multiple visual perspectives in comics storytelling, I would like to briefly discuss one specific device for multiplying perspective, which is the use of embedded photographs in the panel sequence. In the following three examples, the photograph, whether a real photograph incorporated in the story or a graphic rendition of an imaginary photograph, draws attention to the choice of perspective, and the use of juxtaposed perspectives, by multiplying points of view. In thus remediating photographs, narrative comics further pose the question of the specificity of their art form for representing visual experience. Here, comics, so to say, think with their own medium by way of the other medium.

The Australian artist Shaun Tan's graphic novel about immigration, the prize-winning The Arrival (2006), is a story with wordless images. What I would like to highlight in this work are some of the functions of the graphically drawn family photo portrait that we see at various moments of the story. The portrait is pictured at the beginning of the 


\section{2}

narrative and at its end, in addition to when the father of the family is shown looking at it after being separated from his wife and daughter in a strange new land. First of all, it is significant that this image draws our attention to the characters, who gaze directly at us, and furthermore that this exchange of gazes is seen through the father's eyes. The subjective aspect of the viewpoint is revealed by the father's hand, which we see taking the picture from the shelf and packing it in his luggage. Besides the subjectivity of the gaze, the introduction of the portrait reveals the emotional intensity that accompanies all the later viewings of the image. Another important aspect of the viewing is that it emphasises the impression of a photographic frame, that is, the sense in which all the panel images in Tan's book more or less resemble old black-and-white photographs. The effect is even more prominent in the father's passport photo and the worn-out panel frames in some of the embedded stories, which recall an old photo album.

Jarmo Mäkilä's fictional portrait of an artist, Taxi van Goghin korvaan (A Taxi into Van Gogh's Ear, 2008), the second part in a trilogy by this Finnish painter, is a complex mixture of childhood memories, hallucinations, and a Dantesque journey into the world of the dead. The main character is split into different personalities: a lonely boy, a clown, and an adult man, the artist called Itikka (meaning 'bug' or 'mosquito'), who all seem to live in two different realities at the same time, the world of the living and the world of the dead. At one moment in the narrative, the artist is lost somewhere in a forest where he finds a photograph in his pocket depicting himself as a young boy with his parents and a girl he loved (Figure 6.1). The incorporation of a graphic version of this photograph again multiplies perspectives by introducing subjectivity into the sequence, and underlines the interpenetration of different levels of reality and memory. The effect continues in the next panel, spread over a whole page. Here, we are suddenly taken, as if momentarily sharing the main character's gaze, into another world where we meet the artist drifting in space amidst toys on a plastic rowing boat. The multiplication of perspectives leads us to other levels of experience, contrasted with the verbal focalisation of the story, which gradually moves from first- to third-person narration.

Finally, Emmanuel Guibert and Frédéric Lemercier's travel book trilogy Le photographe (2003-2006, translated as The Photographer: Into War-Torn Afghanistan with Doctors Without Borders) raises quite explicit questions about media hybridity and the realism of the photograph. The Photographer is based on a true story written by the French photographer Didier Lefèvre about a Doctors Without Borders mission in Afghanistan in the late 1980s. Almost every page and spread of this story includes Lefèvre's actual photographs from the dangerous journey, and sometimes a whole page or a double spread is composed just from them. The graphic image and the photograph have pretty much equal weight throughout the book (Figure 6.2). 


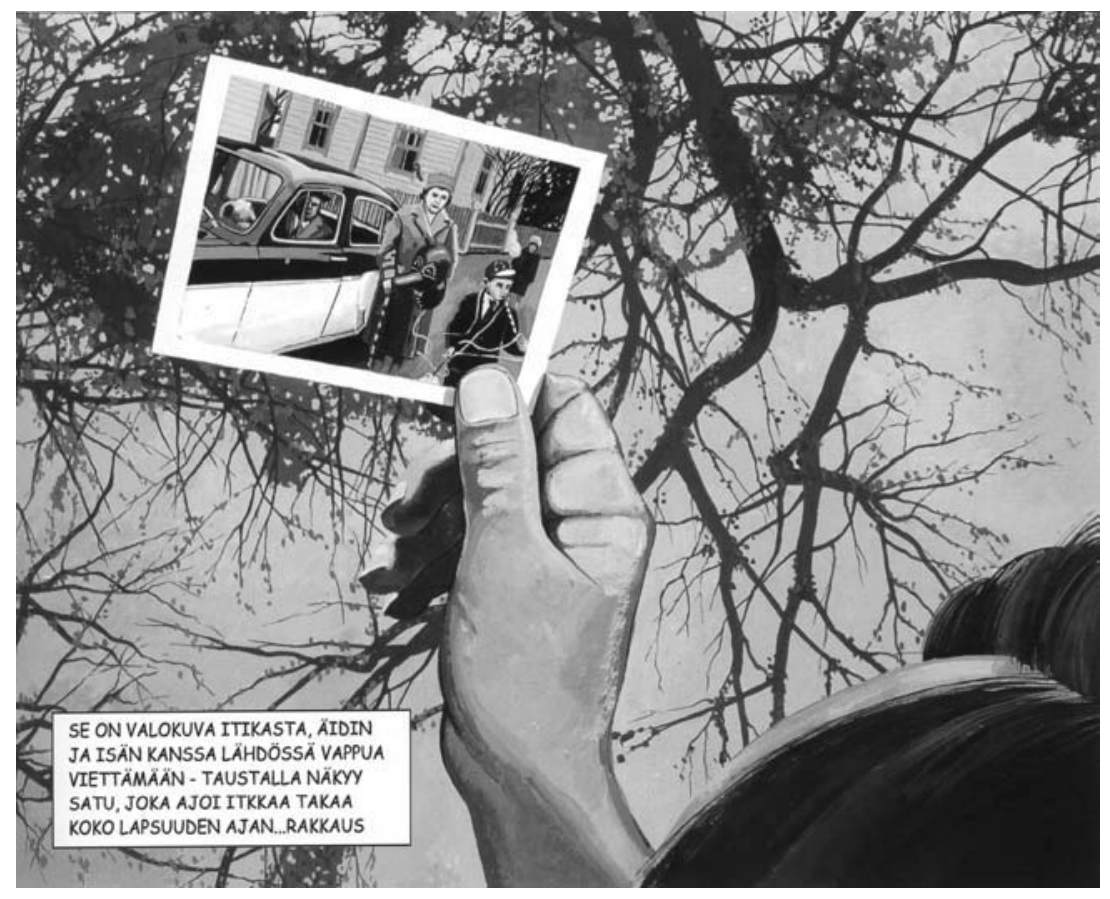

Figure 6.1 Jarmo Mäkilä. Taxi van Goghin korvaan (2008) (C Jarmo Mäkilä.

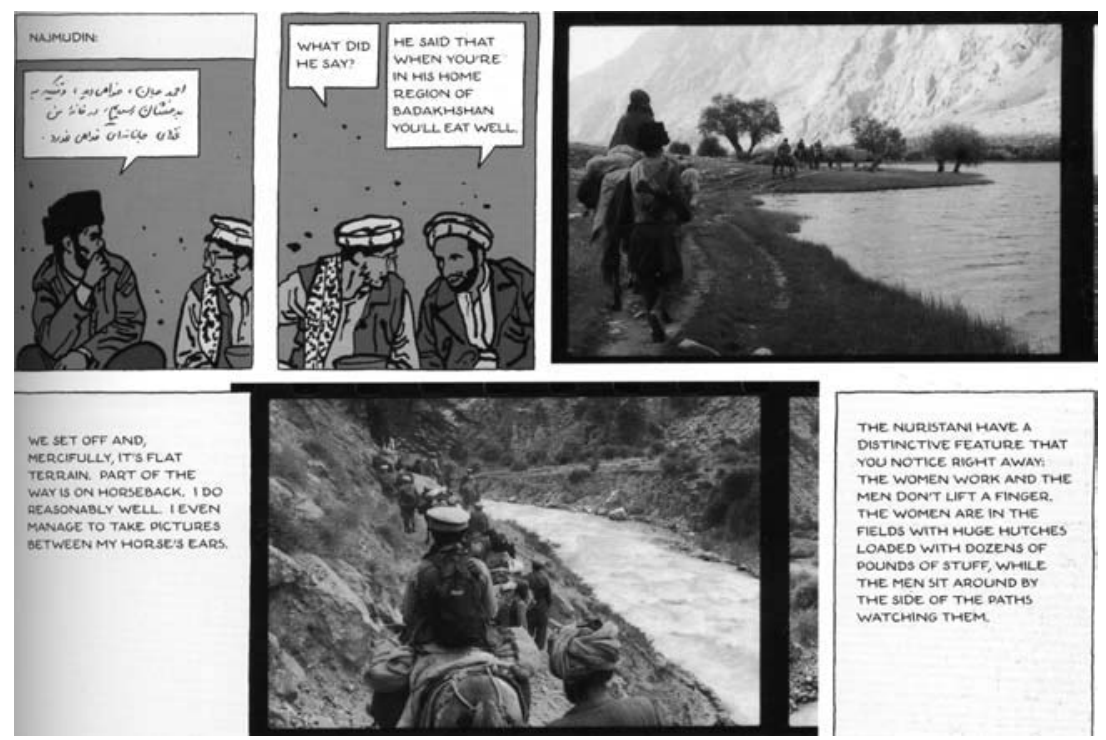

Figure 6.2 Emmanuel Guibert, Frédéric Lemercier, and Didier Lefèvre. The Photographer () 2009 by First Second. 
What interests me in this last case is not so much the remediation of the photograph as part of comics storytelling-a process by which comics, as in the two previous examples, refashion photographs, break them into a story and thus, in a sense, try to improve upon them-but a more balanced dialogue between the media, where photographs have an impact on the graphic images, and vice versa. On the one hand, the various instant moments that are captured by the camera and included in Le photographe are given narrative shape and meaning by the graphic images and the first person verbal narration that accompany and surround them. The photographer, his medium, and his equipment are literally made part of the story. On the other hand, the integration of photographs has an obvious, many-sided impact on the graphic images and their sense of veracity. This is not so much that the photographs give an air of authenticity to the graphic panels even if this might happen as well, but that they make the drawn images look more like photographs, like an instant shot.

The effect of this intermedial composition may perhaps appear awkward to eyes unaccustomed to it, but the alternation between the media is itself also worth attention. Neither medium serves as the other's relay or amplifier, but both move the story forward. Sometimes one of them dominates a single page, a double spread, or an episode. The photographic images are interwoven with the graphic panels in the sequential order of the narrative, while they also preserve something of their status as individual images illuminating an instant. The photos enjoy a certain distance from the verbal 'voice-over' in the narrative boxes. The same is not afforded to the drawn images. While verbal narration often accompanies the photographs, the photographic images never include dialogue, and the verbal narration next to them remains carefully separated, not part of the frame.

The question of veracity is further complicated by the graphic nature of the images involving real objects and people. The reality of the point of observation and the field of vision in comics storytelling, even if it is nonfiction, is necessarily make-believe. No one ever saw the world as drawn, or as caricature, no matter how detailed or instantaneous the graphic line may be. Naturally, no one sees the world as photographs either, but there is a necessary graphic distance from the world that is represented. As happens in Le photographe, the distance between different kinds of image and the perspectives they offer can provide nonfiction with particular self-critical potential, pointing out that the reality in pictures is always mediated. In Le photographe, the hybridity and the multiplication of visual points of view highlight the meaning of the perspective both for narrative organisation and for the sense of reality. 


\section{Scale of (Visual) Focal Points between Internal and Non-Character-Bound Positions}

In visual storytelling, in general, impersonal focalisation is a much more prominent and broader phenomenon than in literary narratives. ${ }^{11}$ Visual narration across media, from films to photo stories and picture books, can deploy a broad scale of techniques between more or less personal or impersonal perspectives, from panoramic views that no person could see to partly sharing subjective vision and to a point-of-view image. Comics storytelling, when it comes to the visual perspective of the narrative, uses an extremely complex scale of potential intermediate positions between subjective or internal focalisation at one end, and clearly noncharacter-bound perspective or external focalisation at the other. This scale, however, is not organised in a strict external/internal, or reflector character/narrator binary, as may be the case in literary narratives. Rather, it reflects varying degrees of congruence and divergence between a character's point of view and the reference world of the narrative, as well as the fact that comics can use internal and external viewpoints at the same time. In a great number of externally focalised images, no supposition of an individualised perception can be made. For these panels, we may postulate some anonymous agent, a non-character-bound external focaliser. What makes subjectivity particularly flexible in comics is also the capacity to alter the perspective from one panel to another. However, contextual information, based on our evaluation of the narrative sequence of panels, the layout, or the relationship between words and images, becomes important especially when the degree of subjectivity changes from one panel to another. Only contextual information of a larger narrative sequence may allow us to determine whether a certain perspective moves towards or away from subjective perception.

The techniques for subjectifying narrative perspective in comics include different formal choices with regard to the position, angle, field (or scope), distance from the regarded object(s), depth, and focus of vision-the latter involving, for instance, foreground-background relations, level of specificity, and detail. ${ }^{12}$ The most common techniques of subjective focus of perception, in comics as in film, comprise the various ways in which the character's positioning in a given image or sequencein relation to the frame and what is shown in the images-suggests a subjective narrative perspective. Such techniques comprise, for instance, the point-of-view (POV) image (the impression that the reader shares the field of vision with a particular character), the positioning of the viewpoint on the eye level of the characters (without implying an actual POV image), the over-the-shoulder image, the gaze image (showing a character looking at something), the eyeline image/match cut (a combination of 
a gaze image that is preceded or followed by a point-of-view image), and the reaction image (a character reacting to what he has just seen).

Equally, subjective vision can be suggested by the exaggeration of the foreground of the image, by particular optical effects, such as an out-offocus image that is related to someone's mental condition, and that can be used to create a sense of a perception image, ${ }^{13}$ as well as by visual effects or signs of the observer's physical presence, such as a shadow, a speech balloon, or parts of the body, positioned in the foreground and close to the image frame. All these effects can be accentuated by the context of other panels and the page layout, where a field of vision may be connected to someone looking or speaking. All "objective" or descriptive images may also be subjectified or have this effect when juxtaposed with a gaze image, a reaction image, or other clear indications of gaze and subjectivity in the panel sequence.

To better describe this multitude of positions classifiable as 'vision with' or 'vision from behind', we can first point out that comics storytelling has at its disposal most of the cinematic techniques for getting close to a specific subjective point of view, from suggesting a subjective perspective to adopting and wholly assuming it. We can use as our starting point Mandfred Jahn's (2003) list of the five most important subjectifying filmic devices. The typology, however, requires us to change the filmic term 'shot' into 'panel' or 'image' and make some further medium-specific adjustments. It needs to be stressed that the equation between a shot and a graphic image in comics is not unproblematic since a shot is a sequence of frames ${ }^{14}$ and a panel is (mainly) a single image. Moreover, the perspectival function of a single image in comics may often be specified only in the context of other images. The categories, however, are illustrative of the general options in this regard:

1 the point-of-view image (or sequence) (POV)

2 the gaze image

3 the eyeline image/match cut

4 the over-the-shoulder image

5 the reaction image.

First, the point-of-view image (or sequence) (POV) is the most internal, direct, and subjective perspective. It assumes the viewer's position; the image frame functions as the representation of someone's gaze and a field of vision. Yet we must also add that the presumed subjectivity of a POV image always involves an interpretive move and potential ambiguity: how do we know that a certain perspective in a panel or an image sequence belongs to someone? One clear cue for this is the positioning of the viewpoint on the eye level of the characters even if it is insufficient in itself to imply a POV image. In comics, more context can be given in a sequence or other group of panels, including for instance a gaze image or 
indications about the viewer's position and relation to the field of vision. The technique, however, also cannot be used extensively for obvious reasons, as studies of 'subjective camera' in film or the picture book have shown. The main difficulty is that the character whose perception we share could never appear in any picture except in a mirror or a reflection.

What film studies calls the perception shot is also always possible in comics. This is a specific type of POV shot that, as Branigan defines it $(1984,81)$, reveals the mental condition of someone looking at something. A perception image in comics most often shows difficulty in seeing or the viewer's heightened attention, typically a blurred scene that presents the vision of a drunkard or someone who is fatigued. Another option in this respect is the highlighting of something in the image by graphic means as the focus of a character's attention. This is realised, for instance, in a sequence of two pages in Scott McCloud's The Sculptor, where the protagonist, David Smith's focus of attention on a young woman is indicated by two visual means that operate together: the POV image, where the readers share David's perspective, and the colour contrast of the female figure's bold black colour against the faint blue-toned grey background scene.

Moreover, the way that something like a city scene or a landscape is visually rendered, by using conventional implications of colours, varying intensities of lines and shading, or patterns of shapes, can suggest a subjective viewpoint. In this respect, there may be great differences between different visual media in terms of technique even if the basic function of the perception image, sequence, or shot is similar. The tone of a film narrative, for instance, is the result of a wide variety of stylistic choices encompassing lighting, cinematography, mise en scène, and the editing of both the image and sound tracks, while cartoonists can use the panel setup and page layout, the frame shape and size, colouring, and the graphic line for similar purposes.

Second, the gaze image is a picture of a character looking at something. More precisely, this is an image with external perspective that shows a character looking at something that cannot be seen in the same image, thus drawing our attention to perception. Generally speaking, in viewing visual stories we continually make inferences from people's looks, gazes, glances, and facial expressions so as to have access to their subjective states even if these states may remain fairly indeterminate. Another important category of looking and gazing in comics is the direct gaze, meaning participants in pictures who look directly at the viewer. We could recall here the theoretical division into 'demand' and 'offer' images, as defined by Kress and van Leeuwen (2006, 126-127). Realising a visual 'you' in this way, the 'demand' picture suggests a particular, often genre-specific type of interaction between the picture and the viewer. The technique can also increase narrativity by suggesting a heightened level of involvement for the reader.

Third, the eyeline image/match cut is a combination of a gaze shot that is followed by a POV image. Thus, the gaze image cues the audience 
into interpreting the preceding or the following image as a POV image. The use of image/reverse image technique is also common, as we will see later in this section of the book, in dialogue scenes. Another form of this technique is the impression of a collective vision in which the perspective is not mediated through any one character, but rather falls within the perceptual range of several characters whose gazes and objects of vision alternate in the given scene. ${ }^{15}$

Fourth, the over-the-shoulder image is a less direct and less internal means of subjectification than the point-of-view image. In a film, this means that the camera gets close to, but not fully into the viewing position, or that a camera follows the movements of some character. In comics, the image may be shown, for instance, from behind a character's back, close to the character's viewing position, or in conformity to the character's direction of looking. A sequence of images may also follow a character's action or movement so closely as to suggest that the story follows not just the character's action and movement, but also their perspective. Another similar device is the placement of the character in the image in such a way that his or her position can subjectify the perspective, for instance, when the character's back or side profile is placed beside the edge of the image frame so as to heighten the reader's association with the character's perspective. Likewise, the depiction of the viewer's hands or lower body beside the image frame, or his or her image in a mirror, also points out that we share his or her perspective. The drawing of a character's fingers beside the panel frame, as in the example above from The Arrival, is another pictorial convention that reveals the subjective perspective of the image.

Finally, the fifth subjectifying device is the reaction image, which shows a character reacting to what s/he has just seen. Similar to the gaze image, the reaction image draws our attention to perception, but does so retrospectively, after the act of viewing or perception.

All these techniques and devices amount to an extremely complex scale of intermediate positions between clearly subjective and clearly non-character-bound perspectives. One important conclusion to draw from this is the central role of ambivalent focalisation in the medium. This could also be characterised as the predominance of 'free indirect perception'. The category of 'free indirect perception' originally refers to a kind of 'narrated perception' in literary discourse, which occurs when the narrative clearly describes or implies the perception of a character. A character's mind is thus implied as the perceptual angle of some textual passage, but his or her perceptions are never directly introduced by perception verbs or other linguistic means. ${ }^{16}$

In films and comics alike, similar effects are commonplace. Charles Forceville, for instance, has suggested that studying techniques like character-bound camera movement in terms of free indirect discourse, specifically when such techniques create ambiguity between the external narrative instance and the character, could contribute to a transmedial narratology $(2002,133)$. The free indirect instances in films, which take 
place when audiovisual information is shared between the character and the overall narrative frame, also involve passages when certain things are distilled through a character's perception by means of colour filters, visual distortions, and other such techniques. In comics, as we have already seen above, there are a number of ways by which the narrative can focus on the character's field of vision and make us systematically look either with him or her or look from behind him or her. These techniques may allow the reader to look deeply into the character's field of vision, even limiting the view to the range of perceptions available to some character, while at the same time retaining the sense of a hypothetical viewing position that does not belong to any character. In comics, the spatially determined viewpoint obviously cannot be associated, as it is in narrative film, with the movements of a camera, but this does not make it more personal as such. We watch along with a character, from the character's back, or gain insight into his or her point of view through what we see in other ways, but focalisation is usually not entirely left to the person, or any person.

To briefly return to Shaun Tan's The Arrival, we can detect here a great number of images that imply a strong subjective angle even if there are relatively few clear cases of a POV image or sequence. Consider the end of the episode where the main character, who at this point is still living alone in the foreign country, is invited to have dinner at the home of another immigrant family. In one large image that spreads over a page, we see a scene around the dinner table (Figure 6.3). The following twelve close-up images consist of a series of eyeline matches, leading us from one subjectified field of vision to another, motivated by the direction of the character's gaze in the previous panel, and at the same time restricted to what these characters may see from their respective positions. This creates the effect of intertwined looks around the table that reveal to the viewer things happening from different angles, as if we could alternate between each person's eyes. Yet, while the lonely father's subjective vision is at times strongly implied, especially at the end of the scene when the couple looks directly at him and us, we also see him from the outside. We also observe the other people from angles that could belong to any of the characters present or to no one in particular.
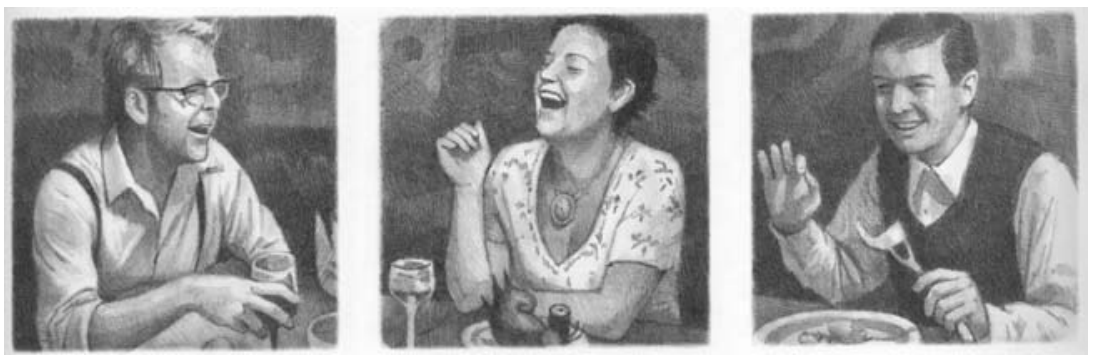

Figure 6.3 From The Arrival by Shaun Tan. Scholastic, Inc./Arthur A. Levine Books. Copyright ( 2006 by Shaun Tan. Reproduced with permission. 
It does not seem necessary to postulate one particular subjective viewpoint in order to understand this scene. Instead, what matters, and what, to some extent, subjectifies all the views at the dinner, is the restriction of visual information to the immediate views of people around the table. This limitation of the perspective, combining a possible gaze image with a potential POV image in a sequence, emphasises the joyful atmosphere and the feeling of sympathy among the members of the party. Instead of any fully subjective, personalised vision, the readers have access to many subjective views. Besides, we may also have access to what literary narratology sometimes calls the empty deictic centre: a position that is clearly on the scene with the characters, but without the possibility or even the need to identify with any of them.

One explanation for this vast scale of varied and combinable options in focalisation is, to follow François Jost $(1983,195)$, the difficulty in designating the degree of deixis in a single image, even when we are dealing with cases of subjective perspective. The non-character-bound perspective, generally speaking, is coded for transparency: while showing and framing a field of vision, it does not necessarily presuppose a human narrator or a reporter. Depending on the narrative context, however, the 'objective' image or sequence can also be marked by a character's or a narrator's subjective field of vision and perception, or encompass it. The default mode for visual focalisation in general seems to be an external viewpoint subjectivised to varying degrees by various visual, stylistic, and compositional techniques.

Another conclusion that we can draw from the importance of the vast range between impersonal and subjective forms of visual focalisation is the way that the intermediate positions may heighten the play of divergence and convergence between words and images. These techniques enable comics storytelling to fully exploit the distance between a self who speaks, a self who sees, and a self who is seen, or the split between a narrating and an experiencing self.

Exempting wordless comics, the effects of all visual techniques of subjectification are accentuated, complemented, and sometimes contrasted by verbal narration. Thus, to understand the degree of subjectivity in focalisation in comics, it is necessary for the reader to process the interaction between 'focalisation markers' at these two levels of narration, the verbal and the visual dimension of narrative representation. Verbal narration may also complicate the establishment of perceptual focalisation. Particularly in cases of split verbal focalisation, in which the same person (or character of fiction) speaks and narrates simultaneously outside and inside the image, the relationship between the visual and the verbal narrative perspectives can become quite complex and dynamic. This is usual in first-person literary narratives, but what is specific to comics in this regard is that the two modes of telling need to be reconciled with two modes of showing: the narrator shown in action and the narrator shown as a narrator, or as someone who is aware of his or her drawn self and role as a narrator. ${ }^{17}$ 


\section{Embedded Focalisation}

The distinction between the source of focalisation and the focalised entity is often relaxed, or remains ambiguous, in the technique of embedded focalisation, which takes similar yet ultimately different forms in comics (and visual storytelling) compared with literary narratives. What Mieke Bal calls an embedding of focalisations involves transference between subject and object positions along different diegetic levels in a narrative, for instance when some narrative object (the object of narrating, focalising, or acting) becomes the subject (the narrator, the focaliser, or the principal actor) of the following level (Bal 1981, 45). Another possibility of the same phenomenon is that the external focaliser in a narrative watches "along with a person" but without leaving focalisation entirely to this character (Bal 1997, 159). ${ }^{18}$ In visual terms, this may be likened to the many techniques that subjectify the field of vision without turning the image into a pure subjective image, as discussed above.

It is difficult to find any literary equivalent for the techniques of perspective filtering in Calvin and Hobbes, where the mere presence of some figures in the scene may indicate a change in perspective. Most importantly, when only Calvin and Hobbes are present in the image, Hobbes is not drawn as a stuffed animal but as a living being, an anthropomorphised tiger. This is one of several sophisticated means of focalisation in this series, and not only different in degree from the techniques used in literary narratives. The effect is based, however, not so much on inferring how graphic style reveals a narratorial or authorial perspective that might embed other perspectives, but on visual information about the supposed focaliser in the image. The child centre of attention (Calvin) is simultaneously often shown from the outside. Hobbes is depicted in a particular way in particular images-either alive or not alive-since we have come to learn that certain people, whom we also see present in the image (Calvin or his parents or someone else), perceive Hobbes in this way. What Calvin and Hobbes shows us so well is that, in visual narratives such as comics, internal and external focalisers can appear simultaneously, embedded in a literal sense, at different points inside and outside the image frame.

\section{Conclusion}

In order to develop a narratological understanding of perspective in comics, it is important not only to find common ground between different narrative media with regard to their techniques of perspective-taking, but also to develop ways to explain the fundamental differences between different narrative media in these techniques. Emphasising the issue of perceptual focalisation, I have posed a number of medium-specific questions that should be accounted for in order for us to conceive a more 


\section{2}

comprehensive narratology of perspective and narrative mediation in comics. These include, in particular, the problems of epistemic access to the point of perception and of what is perceived, pertaining to the spatially determined point of perspective and the evaluation of the degree of deixis and subjectivity in graphic images, as well as the difficulty of distinguishing between the focaliser and the focalised in some cases, and the implications of that difficulty for the theory of focalisation.

These are not the only important questions to ask about the possibilities of analysing focalisation in narrative comics, but they are ones that are of great value for narrative theory that seeks to be relevant in this field. In the narratology of comics, problems will arise if we assume that notions of narrative perspective, transmission, and mediacy can be transferred from one medium to another without due modification. Yet, we may also confront problems if we are unwilling to see some of the crucial similarities that exist across narrative media. The latter include, for instance, the importance of a situated focus, spatial representation and perceptual information, the relation between perception and cognition in the representation of fictional characters' minds, and the variable quality of types of focalisation in narratives across the media.

\section{Notes}

1 Indications of the viewer's identity, location, and time of viewing within the given image or sequence. In linguistics, deictics are most commonly defined as linguistic expressions, such as personal and demonstrative pronouns, whose referent has to be found in the situation relative to their act of utterance. See, for instance, Lyons (1977, 636-690).

2 See Bal $(1997,143)$.

3 Genette's conclusion, however, disagrees with those views that emphasise the necessity of spatio-temporal positioning in all narratives, such as Bortolussi and Dixon's psychological viewpoint (2003, 166-178). For Genette, focalisation is merely a device that may or may not be employed in literary narratives, and the question of the literal location of the point of view is often of negligible importance $(1988,76)$.

4 See, for instance, Deleyto $(1996,222)$ and Gaut $(2004,247-248)$, on what he calls the intrinsic perspective of the film image.

5 See also Jost (1983, 194-195) on this question in film narration.

6 The Marey effect is named after the French physicist Étienne-Jules Marey's (1830-1904) chronophotography. Marey photographed the movements of men and animals several times in a second with his chronophotographic gun, thus allowing the decomposition of movement in elementary phases (recorded on the same image or in several frames of print).

7 Genette's original formulation does not distinguish between focalisation as the "focus of perception" or focalisation as the "selection of narrative information" (1988, 64, 74). For a discussion of this fundamental contradiction, see Jesch and Stein (2009). In comics studies, Badman (2010) follows Shlomith Rimmon-Kenan's distinction between perceptual, psychological, and ideological orientation; Horstkotte and Pedri's definition is similar $(2011,331)$. 
8 See Miller (2007, 106, 109), Badman (2010), Groensteen (2011), and Mikkonen (2013).

9 Groensteen prefers the notion foyer perceptif to ocularisation so as to avoid a too-close association with cinematic devices such as the viewfinder, the objective, and the camera eye (2011, 90-91). However, 'focus of perception' or 'perceptual focus', which are possible translations of 'foyer perceptif', are too limiting for my purposes. Bal's definition of focalisation as "the relation between the vision and that which is 'seen,' perceived" (1997, 142, emphasis added), is more encompassing.

10 As William Nelles points out (1990, 371), Genette has explicitly recognized this difficulty in regard to the category of zero (free) focalisation. He writes, "the right formula would be: zero focalisation = variable, and sometimes zero, focalisation. Here as elsewhere, the choice is purely operational" $(1988,74)$.

11 Much of the critique of anthropomorphism in focalisation theory, meaning the assumption that the focaliser would always be a person who perceives, has been based on the insight that we need to pay more attention to techniques of impersonal perspectives.

12 Corresponding in large part to what cognitive linguists call perspectiverelated parameters in narrative contexts and that include: the location of a perspective point with a "referent scene", the distance of a perspective point from the regarded scene, the perspectival mode, and the direction of viewing. See, for instance, Talmy (2000, 311-344), and also discussed in Herman (2009b, 128-132).

13 Branigan distinguishes the perception shot from the point-of-view image, suggesting that the former includes an indication of a character's mental condition, "a signifier of mental condition has been added to an optical POV" $(1984,80)$.

14 A shot "is a sequence of frames filmed in a continuous (uninterrupted) take of a camera" (Jahn 2003).

15 See also my discussion of a passage in Daniel Clowes's Ghost World from this perspective in Mikkonen (2011b, 647-650).

16 See Chatman $(1978,204)$ and Mikkonen $(2008,301-321)$.

17 The insufficiency of the distinction between narration and focalisation in film narratology is also reflected in the need to add a third component to the model. Branigan has argued that there are three distinct types of narration in films-narration, action, and focalisation-that describe "how knowledge may be stated, or obtained" $(1992,105)$.

18 However, Genette rejects the notion of embedded focalisation in literature, claiming that the focus of the narrative cannot be at two points simultaneously, even if a narrative mentions that a glance "perceives another glance" $(1988,77)$. What Bal defines as embedded focalisation, Genette would call displacement of focus $(1988,76)$. See also my critique of O'Neill's transmedial version of the notion of embedded focalisation (Mikkonen 2011b). 


\section{Characterisation in Comics}

How I want thee, humorous Hogarth!

Thou, I hear, a pleasant Rogue art.

Were but you and I acquainted, Every Monster should be painted:

You should try your graving Tools

On this odious Group of Fools;

Draw the Beasts as I describe them:

Form their Features while I gibe 'em;

Draw them like; for I assure you,

You will need no Car'catura;

Draw them so that we may trace

All the Soul in every Face.

-Jonathan Swift, "A Character, Panegyric, and Description of the Legion Club" (1736/1998, 229-230)

One indication of the centrality of the character in Western fictional imagination is the naming of major works according to the protagonist, from Odysseus and Aeneas to Don Quixote, Tristram Shandy and Anna Karenina, and from Little Nemo and Adamson to Corto Maltese, Little Archie, Bamse, and Gaston Lagaffe. These classics are known for their characters, and the protagonist's name serves as a kind of reading instruction: their behaviour, fate, or experience is the focus of the story. Another indication of the importance of characters in fiction, regardless of media, is their semi-independence: we can refer to them and their unique features independently of the stories where they have been created. Little Red Riding Hood, Robinson Crusoe, Dracula, Peter Pan, Sherlock Holmes, Pippi Longstocking, James Bond, Mary Poppins, Tintin, Asterix, Batman and Superman, and Mickey Mouse and Donald Duck are not only characters in well-known fairy tales, novels, films, and comics, but widely known figures in our culture.

Characters are a persistent feature in comics, but are there comics without characters? In some non-person comics, or it-narratives, ${ }^{1}$ such as Intérieurs by Régis Franc (1979), The Short History of America by Robert Crumb (1979), and The Cage by Martin Vaughn-James (1975), ${ }^{2}$ 
the only focus and 'engine' of the story seems to be the changing décor and the world of places and things held in the field of vision. Yet, the rejection of characters in these works is not as absolute as may first seem. The human figures who can be seen as part of the images in Crumb's short American history ${ }^{3}$ are not characters in the story, but indicative of the signs of human imprint and influence: how people shape the environment over a long period of time. At the same time, a human voice plays a seminal role in the two other examples: Franc's and Vaugh-James's comics suggest a character's presence by verbal narration in the first person. In fact, in Franc's Intérieurs, the first-person narration creates a strong sense of a continuing consciousness frame, accompanied by a subjective focus of perception, for the entire story. The narrator, who has lived in the rooms of a small apartment that is the visual focus in the story, gives meaning to the interiors and objects of the flat through his contemplation, memories, and experience. Even if he is a mere voice, he clearly is a character in his own story.

In it-narratives in comics, there is considerable variety in the way in which the narrating voice or the perspective can be personified, that is, attributed personal properties. In Woodrow Phoenix's Rumble Strip (2008), where the only human-like characters in the images are the figures in traffic lights and signs, the narrating consciousness may be associated with the actual author. At the same time, the dynamic narrative situation of this work complicates our evaluation of the identity of the narrating voice. Most of the book is told in you-narration, where the pronoun you hovers between the reference to the actual reader, an implied audience and a second-person character. At the end of the book, by contrast, the narrator shifts from the impersonal mode of argumentation, concerning wasteful road accidents, injuries, and deaths caused by reckless drivers, to first-person narration by telling a story of how he was nearly killed in his car on a motorway from London to Brighton. What challenges the concept of character in this case, however, is not only that no characters are seen in the images, but also that much of the book is not framed as a fiction or even as a narrative, despite its story-like situations, scenarios, and case histories of road accidents-Rumble Strip is rather an argumentation, or an essay, about car culture and road deaths in the author's own name. The category of the character tends to connote fictiveness: characters are an element of a composition; they do not exist as persons even if they are often person-like.

What is important in all of these four it-narratives (Crumb, Franc, Vaughn-James, and Phoenix) from the viewpoint of characterisation is that the spaces that are the focus of the images are strongly marked by human experience, memory, and involvement. The lyrical captions in Vaughn-James's The Cage do not directly refer to any speaking or narrating subject even if they clearly suggest that some human consciousness is filtering the images. The lack of characters functions differently here 
from the other examples, in that we can ask if The Cage is a narrative at all. Might it not be a visual poem, or a lyric comic featuring a series of tableaux that involve various abandoned spaces only loosely connected with each other? ${ }^{4}$ The lyrical captions in the work are strongly indicative of a human consciousness (or several characters' minds), but they do not suggest a continuing consciousness frame that could easily be conceived in terms of narrative cohesion.

In other comics, buildings, objects, or streets have been conceived as characters. For instance, the apartment building where the protagonist of Chris Ware's Building Stories lives, has thoughts and emotions, and Danny the Street in Grant Morrison and Brendan McCarthy's "Doom Patrol", an actual street, is a sentient character with superpowers. Similarly, in Jeanne Puchol's it-narrative Dessous Troublants (1986), the furniture shown in a room has a subjective voice that refers to personal memories and experiences. Also in Wally Wood's adaptation of Ray Bradbury's dystopian short story “There Will Come Soft Rains” (1950), which describes a series of events that take place in the rooms of an automated house during a day in 2026, the story does not feature any humanlike characters. In this case, the narrator's continuous reporting voice, which is external to the story world, is remarkably impersonal: the captions merely describe the evolving events, or indicate the present time and place of the given panel. Nevertheless, the focus on the automated house that has several humanlike qualities-it has a voice in the automated walls and some of its appliances, it has a sense of time, it dislikes dirt, fears fire, and so on-allows the reader to imagine the house as a potential character who is at least to some degree, no matter how illusorily, a sentient and anthropomorphic being.

Drawing on these examples of it-narratives, we can identify three important aspects about characterisation in comics. First of all, it is difficult to tell a story without characters. A mere voice, a subjective perspective in the image, a caption, or a place that is followed, may be perceived as an impression of consciousness and a mental state, which can then be attributed to a potential character. Second, the function of voice and perspective as indicators of subjectivity and characterhood open the question of how to define a character in comics in the first place. It is important to note how comics vary greatly in this respect. On the one hand, personal traits can be expressed predominantly by visual means of external description, or through dialogue, and the representation of mental states can remain implicit or less relevant. On the other hand, mental states and verbal narration can also be the main focus of the story. Third, the limits of the character category in comics have, in some cases, less to do with the characters' visibility and continuity in the images, or the ambiguity of their consciousness or physical shape, than the genre in question. Nonfiction comics, as well as many non-narrative forms of comics, such as lyrical, pedagogical, 
and abstract comics, do not necessarily presuppose the category of the character, despite their possible focus on mental states, consciousness, and subjective experience.

\section{The Concept of Character}

From a narratological perspective, the concept of character is truly multidimensional: characters in fiction are at once entities of the storyworld, elements of a narrative composition, and a means for telling the story. The multidimensionality of the concept has posed significant challenges to narratological theoretisation in the past, reflected in disagreements about the meaning and relevance of the category. One reason for the debate is that the conception of characters in fiction is dependent not only on artistic devices of characterisation but also on genre and medium-specific conventions of storytelling, as well as models of real-world people and their typical situations. ${ }^{5}$ It may then be difficult to distinguish character as a textual component from person as a kind of cognitive model.

Earlier in this book, I have discussed characters mainly in terms of their function in narrative mediation and composition, as important means of connectivity and units of attention in narrative comics. What was left out from this discussion was the question of characterisation, that is, the creation of person-like qualities, and the understanding of the personality traits of fictional characters. Fotis Jannidis defines characterisation as the process of "ascribing information to an agent in the text so as to provide a character in the storyworld with a certain property or properties, a process often referred to as ascribing a property to a character" (2015). In other words, the process of characterisation presupposes that characters are agents that have properties. In this capacity, characters are person-like, i.e. they resemble a person in the real world, or in previous fictions, at least to some extent.

Not all narratologists have been convinced, however, that the question of characterisation belongs to narratology at all. Some have limited the interest in the category of the character to the sense of actants, that is, the study of characters as structural roles in the narrative composition, such as heroes and villains that fulfil a certain plot function. Gérard Genette, in turn, has conceived the issue of character as a matter of story content: what the story is about instead of how it is told. More precisely, Genette defines characterisation as "the technique of constituting characters with narrative texts" $(1988,136)$, and that is constituted of various narrative devices that are not specific to it, such as denomination, description, focalisation, and speech and thought representation (ibid.). Therefore, for Genette, characterisation is a particular semantic effect that should not be privileged over other effects. For instance, if the reader's knowledge of a particular character's personal traits, intention, 
experience, perspective, or worldview is revealed in part through the techniques of focalisation, description and speech, and thought representation, we should privilege the study of those narrative techniques instead of the study of the character effect. ${ }^{6}$

Yet, one rationale for arguing that the question of characterisation indeed belongs to narratology, and that the category of the character should not be reduced to narrative techniques or effects of the story content, is that characterisation cannot always be that easily decomposed into narrative devices without losing sight of a crucially important element of narrative fiction. The study of characterisation allows us to focus on a vital aspect of narrativity, which is the capacity of fictional characters to inspire a narrative response. ${ }^{7}$

In what follows, I will ask how comics may prompt their readers to ascribe properties to characters. In this investigation, I will consider the main parameters of characterisation, especially with regard to the characters' mimetic (realist) function, that is, how they can be perceived as possible anthropomorphic persons. Subsequently, I will discuss the way in which a character in comics may achieve a sense of psychological complexity (to be defined later). One of the most used and useful approaches in this respect has been the rhetorical narrative model that addresses three aspects of characterisation. This model, developed by James Phelan on the basis of earlier approaches and taxonomies in literary studies, distinguishes between the character's mimetic (character as person, as images of possible people), thematic (character as idea), and synthetic (character as artificial construct) dimension. The premise in Phelan's theory is that all these elements are present at some level in all characters in fiction, but they may be more or less developed or underscored. ${ }^{8}$ For instance, even the most realistic character in comics is an artificial construct at some level, made of drawn images and words, but that artificiality can be more or less covert. The meaning of the distinction is that narrative texts make different applications of these dimensions and can change their relation as the story progresses, increasing and decreasing the reader's interest in the reality of the character, a particular theme or some aspect of the composition.

Before moving forward, however, it needs to be emphasised that to focus on characters in narrative comics is always a matter of taking a particular perspective on certain kinds of stories. The idea of characters as agents that have properties is dependent, on the one hand, on the kind of narrative that we are reading and, on the other hand, on how we focus our attention as readers, such as how much we pay attention to characters instead of plot, theme, idea, historical context, or something else. In other words, the focus on characterisation necessarily isolates the character as a separate category.

Most narrative theory agrees that character and action (or event) are interdependent elements in fiction and that for that reason their 
distinction always remains to some extent arbitrary. ${ }^{9}$ The characters' actions create events and move the plot forward, and the meaning of an event is typically filtered through what we know about the participants and how they perceive the event. The events that are related in the story, in turn, can reveal and illustrate the agents' properties. Likewise, it may be difficult to think of characters as distinct entities in relation to first-person narrative fiction where the narrator-character tells his or her story. When the whole narrative is indicative of the narrator-character's properties, focussed on the narrator's speech and thought, it may indeed be more meaningful to investigate the narrative techniques of voice, style, perspective, or the relationship between the time of narration and the time of the events, than to study the narrator as a character. The relevance of the concept of character in first-person narration will ultimately depend on the individual features of the given story and the kinds of questions we seek to ask from it.

In the following sections, I will focus on characterisation in comics, specifically in the mimetic sense: how do comics prompt the readers to ascribe mimetic properties, comparable to some extent with real-life persons? Moreover, how can comics prompt us to ascribe such properties to entities with qualities that are strikingly dissimilar from humanlike persons? The characters' thematic function-how do comics associate characters with certain thematic properties?-will only be discussed briefly. It is, nevertheless, important to emphasise that while characters in comics can represent a theme, an allegory, or an ideology perhaps similarly to the way in which real people may symbolise an idea, an ideology, or a world view, types and caricature play a noticeably more significant role in this medium than in many other forms of representing person-like characters or real people.

\section{Identifying Characters in Comics}

The establishing of a person-like character, or a character as a possible person, involves a set of basic expectations about what characters are, regardless of the narrative medium. This is, to a large extent, dependent on the reader's knowledge about real people, their minds and behaviour. Fotis Jannidis has referred to these expectations by using the concept of basis type. This means the presupposition that a character has an inside and outside, or more precisely that a character has, on the one hand, an invisible "inside", which is the source of all cognition, intentions, wishes, emotions, and, on the other hand, a visible "outside", which can be perceived (Jannidis 2015). Therefore, what distinguishes characters from things and other entities is that the reader can attribute to characters anthropomorphic and anthropoid qualities, such as thoughts and emotions. Subsequently, the recognition of mental states in fictional entities invites the making of the fundamental distinction between 
human-like persons, or anthropomorphic beings, on the one hand, and things, or non-personal entities on the other. Jannidis specifies, however, that all aspects of the basis type can be negated for a specific character in a particular narrative, but either this is done explicitly or it results from generic conventions that enable the treatment of characters in this way (ibid.).

But what makes some entity a character in comics, and how can characters be given traits of personality? Moreover, is there something that sets characters and characterisation in comics apart-especially in terms of their mimetic dimension-from characters in other forms of narrative fiction such as prose fiction, picture books, or films?

We may be able shed some light on these questions by comparing the basic forms of characterisation in two adaptations of Dante Alighieri's Inferno, the first part of his famous epic poem The Divine Comedy: Guido Martina and Angelo Bioletto's Mickey's Inferno (L'Inferno di Topolino, 1949), an important milestone among the Italian adaptations of literary classics in the popular series, Topolino, and the American artist Gary Panter's Jimbo's Inferno, a prequel to Jimbo in Purgatory (2004). ${ }^{10}$ The graphic styles, narrative techniques, readership, publication format, and distribution of these two adaptations are radically dissimilar. However, at the same time, there is sufficient similarity in their characterisation, in particular their use of typified and caricature-like characters, for the comparison to be illuminating about certain basic strategies in comics in this regard.

Gary Panter's Jimbo's Inferno features as its protagonist the graphic artist's pug-nosed character called Jimbo, who is often referred to, and perhaps with good reason, as a punk-rock character. ${ }^{11}$ Jimbo's sidekick and guide, and a stand-in for Virgil, the Roman poet, is a character called Valise. Valise is a rectangle box that speaks and hovers above the ground, reminiscent of a large suitcase, a portable stereo system, or possibly a miniature storehouse that also functions as Jimbo's means of transportation. Valise is also referred to as a "self appointed parole robot". The Hell in this version is a Los Angeles shopping mall named Focky Bocky, and Beatrice, Dante's dead loved one, is represented by a group of "cute girls" called Soulpinx Girls. By contrast, Martina and Bioletto's Mickey's Inferno features Mickey Mouse as a Dantesque character-although an actual Dante is also portrayed at the story's end-and Goofy as Virgil.

The first step in the process of understanding characters, regardless of the narrative medium, is the recognition of some entity in the story as a character. For many readers of these adaptations, the identification of the protagonists probably takes place already before the reading. This is due to various extratextual reasons. First, the title and the cover image tell us who the protagonists are. Second, the reference to Dante's Inferno may provide the reader, at least those readers who know something about Dante's work, with other expectations concerning the characters, their situations, and world. Gary Panter, moreover, specifies, in a description 
of "Focky Bocky" that is placed underneath the Table of Contents, what kind of adaptation the reader is about to read:

Don't try to pass a pop quiz on Dante's hell based on a reading of this comic: it won't work. Even though this comic is engorged with Dante's hell and though Jimbo mouths a super-condensed version of what happens in the infierno [sic], canto by canto, characters are fused, action inverted, parodied, subject to mutation by my odd memories and obsessions and my odd whims, sentences are clipped.

Third, the reader may be familiar with the characters, Mickey, Goofy, and Jimbo, from elsewhere, such as from other comics, and can thus expect them to be the focus of the adventure.

The protagonist Jimbo's name is made perfectly clear by the paratexts of the book, including the title and the two subtitles. The subtitle on the cover reads "wherein, Jimbo, led by Valise—city appointed parole robot, enters the vast gloom rock mallscape, Focky Pocky, in pursuit of the Soulpinx", and on the name page: "A Ridiculous Mis-Recounting Of Dante Alighieri's Immortal Inferno In Which Jimbo, Led By Valise, In Pursuit of The Soulpinx, Enters Focky Pocky, Vast Gloomrock Mallscape". Upon reading, then, a global look at the first pages of both stories can verify who the protagonists are: the named figures who are continuously portrayed in the images (Figure 7.1). In Jimbo's Inferno, the protagonist's name is confirmed in the first lines uttered by Valise in response to the question of the whereabouts of Focky Pocky: "Just look over your left shoulder Jimbo" (7). Similarly, Valise's name is given in the dialogue as Jimbo reveals that "Valise, I have some fear of entering Focky Pocky" (8). Furthermore, a few pages later at the beginning of Canto VIII, Valise identifies himself and Jimbo, upon talking to the amphibious vehicle that is to take them across the marsh of Styx, as Jimbo and "his parole Valise" (12).
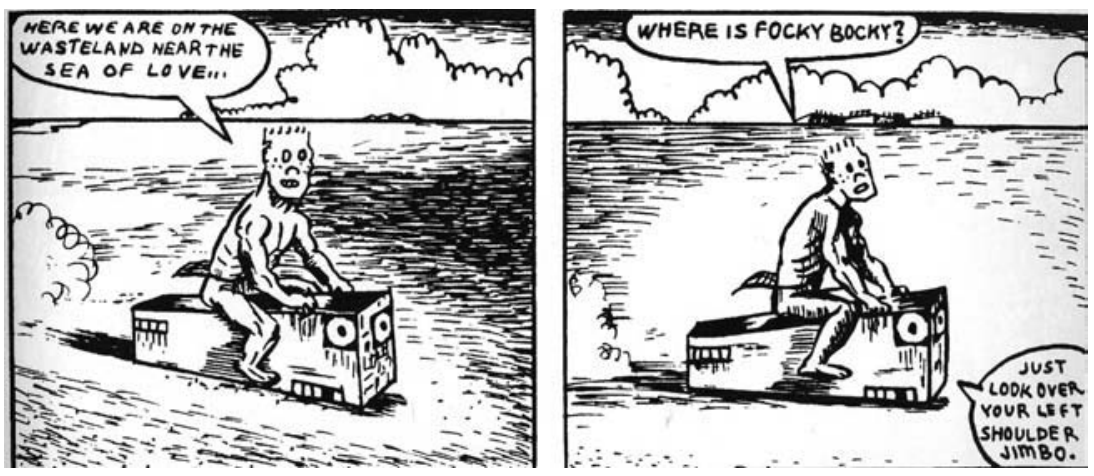

Figure 7.1 Gary Panter. Jimbo’s Inferno (C) 2006 by Gary Panter. 
In the frame narrative of Mickey's Inferno, where Mickey and Goofy are acting in a theatre production of Dante's Divine Comedy, and are then hypnotically transformed into Dante and Virgil and transported into the Underworld, the protagonists' familiar names are given in the first panel. As Mickey and Goofy enter the Hell of the Divine Com$e d y$, they don Dante's and Virgil's clothes and Mickey is also ascribed other aspects of Dante's identity. Mickey wonders to himself: "I saw this creepy forest, as I knew it/From my first canto-I was Dante, right? Or was I? Hmmm! I still felt like a poet... But I'm not Italian, am I?" (Figure 7.2). Mickey's uncertainty about his identity does not last long, however, since a skull falls on his head and he comes back to his senses. Goofy, by contrast, has not lost his old self in the transition and, furthermore, is aware that they have been transported to another world. The situation thus provides the two characters a kind of double identity with their familiar personal traits-parts of their ordinary clothing can also be seen underneath the new cloaks-and with some new features borrowed from Dante's characters, Dante and Virgil.

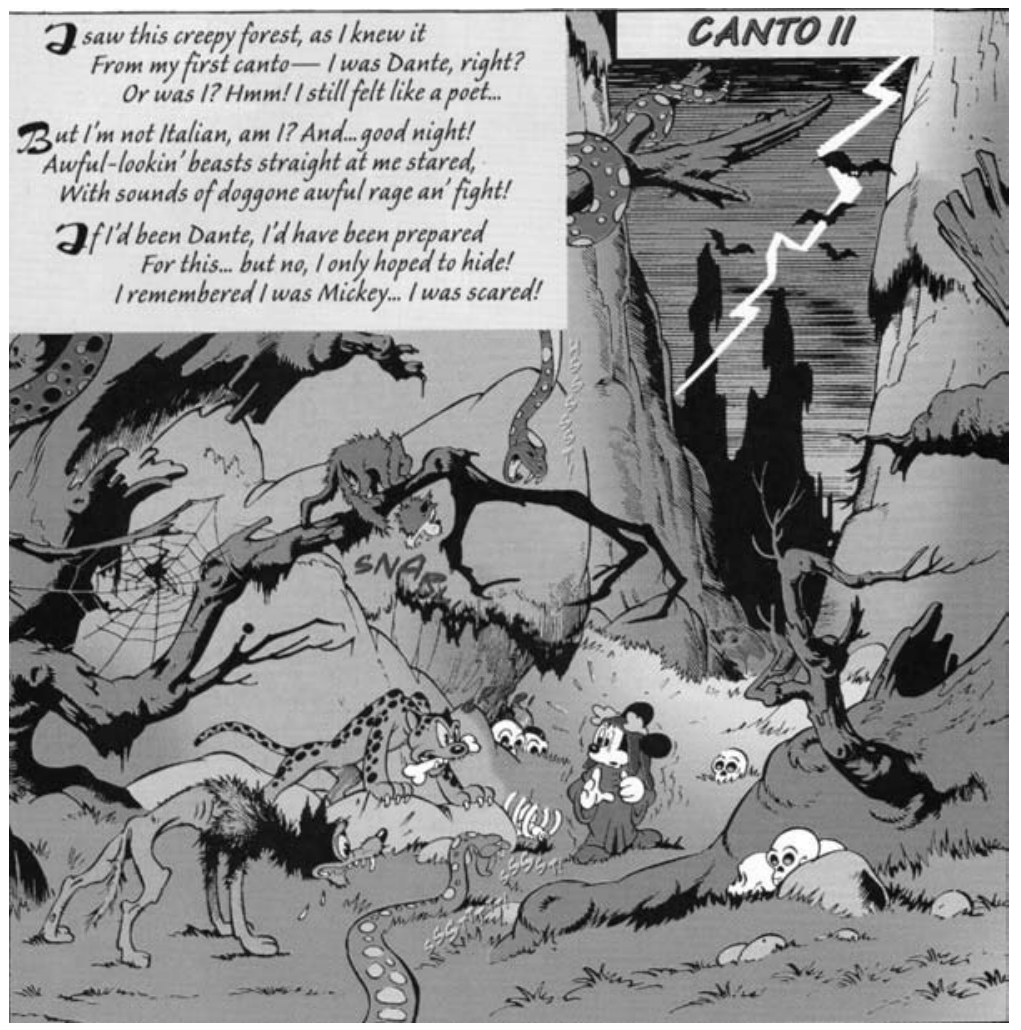

Figure 7.2 Guido Martina and Angelo Bioletto. Mickey's Inferno (1949) (C) Disney. 
The technique of transposition between the fictional worlds thus creates a specific effect of parody, where Disney characters are transported to a well-known literary milieu with some limited changes to their identity - the technique is then re-employed in many later Italian Disney adaptations of literary classics. The presence of various other characters from the Donald Duck universe, such as Pegleg Pete, Zeke Wolf, Pinocchio, and Donald Duck himself further enhances the effect of two superimposed worlds and casts of characters. As Dante, Mickey Mouse maintains his basic characteristics: he continues to be as imaginative, resourceful, persistent, brave, and valiant as always. Yet, his figure also has some added Dantean attributes: Mickey refers to himself and Goofy as poets, and the two protagonists are dressed like Dante and Virgil in the style of Gustave Doré's illustrations.

Characters' names and personal pronouns, regardless of narrative media, are important points of reference in fiction that unite all references and descriptions pertaining to that character. Nevertheless, in written fiction, a name, a personal noun, a name-like description, or other linguistic marker of a character ${ }^{12}$ may play a more seminal role in terms of keeping track of a certain character than in visual narratives. In literature, a proper name provides not only the process of characterisation with a rudimentary but also necessary means of consistency, that is, a point of reference to which properties can be ascribed. Both the implicit means of characterisation, such as the character's actions that suggest traits of personality, and explicit descriptions by the narrator or other characters, can be united in the character's name. In comics, the recognition of the same figure can already fulfil this function to a large extent. The character's continuing presence in the panels serves as a point of reference that helps the reader to construe an entity as a person-like character.

Not much is required of a thing to become a character in comics. Just an abstract shape is sufficient, provided that it behaves to some extent like a person, can speak or think, or engages in goal-oriented action. Think, also, how square shapes, cubes, and boxes have been characters in comics from Ernest Riebe's Mr. Block from the 1910s to Squarehead who was featured in the late 1950s American comic book Cosmo the Merry Martian. In Jimbo's Inferno, Valise is a rectangle robot-looking box, unchanging in outlook, with no other signs of inner life other than his speech-Valise's movement can also be perceived to be mechanical. Valise's comments are often mere instructions to Jimbo, and he may refer to his own thoughts and imagination as "computations". At the same time, Valise can be quite spontaneous, opinionated, ironic, and metaphorical in his responses, and obviously he also speaks and understands speech-all indications of human-like mental states. Valise is also much more reflective and analytical than Jimbo concerning the potential outcomes of his actions. Jimbo, on the other hand, is a man of action and simple pleasures who appears to be a kind of blank canvas with little 
interiority and is mentally clearly more limited than his robot-like counterpart. Despite his person-like appearance, Jimbo is without a sense of a past or much sense of the future, and seems to have a very limited notion about the meaning of his experiences. His simple-mindedness, straightforwardness, and innocence contrast with Valise's knowledge, experience, and occasional complex thoughts. Both of these characters, thus, in their varying ways, challenge the division into a character's exterior and interior qualities.

But let us stop here for a moment: how was I able to claim that these particular traits can be ascribed to Jimbo's and Valise's characters? Beyond the recognition of a basis type, or the identification of a character's bare existence, there are a number of other conditions that affect the recognition of some entity as a character, regardless of the narrative medium. The basic principles of characterisation that narratology has identified in literary narratives are effective here, with the exception that we also need to pay attention to visual cues of characterisation and the interaction between words and images. Mieke Bal, for instance, lists four principles of characterisation, which work together to help the reader to construct an image of a character: repetition, accumulation, relations to other characters (or a character's relation to itself in an earlier phase), and transformations $(2009,127)$. Rimmon-Kenan, in turn, argues that the four main principles of cohesion that help the reader to create a sense of a character's traits in literary fiction are repetition, similarity (or analogy), contrast, and implication $(2003,39)$. It is worth noting that these two models share three principles: repetition, character's relation with other characters, and the question of consistency. ${ }^{13}$

The repetition of any aspect of the character's external feature, behaviour, speech, or thought has the potential to be an indication of personality. This can involve a word, phrase, way of speaking, facial expression, gesture, behavioural pattern, clothing, association with a certain environment, or the like. Also forms of graphic style, such as colour and the graphic line, can describe a particular character and his or her situation. The repetition of Jimbo's spontaneous reactions to things and people—“Ugh!", "Back off mister dim ass!", or "Hey!"—and his constant questions about where they are going and what is happening are indications of a certain innocence and empty canvas-like quality. These short comments are contrasted with Valise's know-how, advice, or ironic and metaphoric comments, such as "If you don't bury your eggs in the sand, you are only a consummate ape of nature" (XXIX canto). Unlike Valise, however, Jimbo is attributed with explicit signs of emotion that can be seen on and around his face, gestures, and posture, such as the character's sense of surprise, shock, fear, and anger that are depicted by lines and beads of sweat around his face. Jimbo's emotional involvement in action is a steady feature in the story, and this may be taken as an indication of a personal trait, such as spontaneity or, again, certain 
simplicity. He never seems to learn, for instance, that unexpected things are bound to happen in Focky Bocky.

The principles of similarity and transformation can be perceived as aspects of the same rule, pertaining to the evaluation of a character's consistency. In this regard, Uri Margolin has helpfully discussed the minimal constitutive conditions of characters under which they can be introduced and sustained, including the principle of consistency. Such conditions include the possibility of assigning at least one property to an individual whenever that character occurs in the text (Margolin 1995, 376-377). Furthermore, in most narratives, this also requires that the character is distinguishable from other individuals, has a coherent set of features (Margolin 2005, 53), and some persisting sense of identity in temporal continuity despite all the changes that the figure may undergo in the course of the narrative. All these conditions can be easily undermined in comics, but such problematisation is likely to be motivated and thematically foregrounded.

Jimbo and Valise are types, unchanging characters who maintain their visual and personal consistency throughout their journey. An unusual indication that Jimbo might have a more conscious intention behind his actions emerges when he states in canto XXVIII that "I come here gyre by gyre to gain experience of the way" (33). The story, however, does not provide us with any proof that Jimbo's mental state would go through changes over the course of the adventure. In Mickey's Inferno, the situation is more complex in that while Mickey and Goofy clearly maintain their basic, unchanging personal traits in the Dantesque Underworld, they also go through a partial transformation under hypnotist Abdul's influence. Mickey's first-person narration, in rhymed prose, also creates a continuous consciousness frame for the narrative that allows us to have some sense of his mental state. At the same time, Mickey's rhyming narration is another indication that he has, indeed, become a poet. However, Goofy also identifies himself with poets as he refers to Dante as a "fellow poet".

The character Valise's box-like shape manifests to us that a character in comics can be invented on the basis of a few recognizable lines and their bodies can be freely shaped for expressive uses. ${ }^{14}$ Moreover, the convention for placing utterances, typically in speech and thought balloons, next to the source of speech has the potential to match some entity directly with a mental state. With regard to anthropomorphic animal figures, such as Mickey and Goofy, the expectation that characters have an "inside" is usually held, and typically made evident by means of dialogue, although that inside may not contain much depth. What is unusual with regard to Mickey's Inferno is that the continuous consciousness frame of this narration reveals aspects of Mickey's mental state, for instance concerning his conviction, but also sometimes uncertainty, that he may be dreaming the underworld adventure and not actually experiencing it. 


\section{Complexity in Comics Characters}

Characters in comics, in general, are sometimes taken to be flatter than in literary fiction. Such a generalisation is unfair, at least from today's perspective. Yet, the claim also holds true for many kinds of comics. From a historical perspective, it can be claimed that, especially before the development of the graphic novel and autobiographical comics in the 1960s, the psychology of characters in comics had not attracted much attention, and the medium as a whole had strongly relied, as it does still, on caricature and type-like characters. Types in comics are not without psychological appeal, since caricature can effectively illustrate forms of behaviour, individual traits, or social roles, but, as Hergé's Tintin also points out to us, despite the realism of the milieu, the development of the protagonist's inner life is often not that important. ${ }^{15}$ One reason for this is simply that so many comics have been targeted at children.

By contrast, it is a generally held notion that today's character-centred graphic novels and narratives, which have an adult audience, can create complex personalities who have psychological depth. We must then ask: How do we come by information in comics that suggests that a given character is an individual with complex mental states?

Before trying to answer this question, it is useful to think of the way in which character's complexity has been defined in narrative theory. For the British writer E. M. Forster, flat characters are constructed around a single idea or quality in their purest form; they can be summed up in a single phrase. By contrast, Forster defined, in his Aspects of the Novel (1927), the round character as someone who is capable of surprising and changing, and thus having "the incalculability of life about it" $(1953,75) .{ }^{16}$ However, for Forster, flatness does not correlate in a simple way with the character's lifelikeness despite his emphasis that round characters are a major achievement of literary modernism. On the contrary, flatness can suggest a kind of lifelikeness of its own. This is exemplified, for instance, in Charles Dickens's protagonists who can be summed up in a sentence, but still have a "wonderful feeling of human depth" in them (Forster 1953, 68). Moreover, flat characters are useful in providing the story with their own atmosphere, and their collision with each other or with round characters may create specific effects of lifelikeness (Forster 1953, 66). Forster further claims that flatness and roundness can occur in the same characters in different parts of the narrative. ${ }^{17}$

Both Mickey and Goofy are clearly flat characters, easily recognised and remembered. It does not make sense to expect them to be lifelike persons. Goofy's dominant trait is that he is clumsy or, perhaps more precisely, that he is foolish in his clumsy behaviour. The character's goofiness is underscored both by his name and appearance: his long legs, ears, and muzzle, big shoes and tall green, or sometimes blue, hat. The same quality can be observed in his behaviour as Goofy regularly gets into trouble due to his rashness and lack of reflection as much as his 
physical shape. Perhaps this is also something to do with the perceived real-world characteristics of large hounds that, with their sometimes amusingly doleful expressions and clumsy antics, might be perceived as somewhat comic and affectionately soppy. ${ }^{18}$ His dominant trait is further associated with another qualification that is equally unchanging: his goofiness is always lovable; his clumsiness and lack of reflection is funny in a sympathetic way. If Goofy stories include exceptions to these basic qualities of the type, they are thematically emphasised and motivated. For instance, while it is not unusual for Goofy to make the right decision and get himself out of trouble, his cleverness is likely to be presented as luck or a happy coincidence rather than the result of conscious reflection.

These recognisable properties are simultaneously inner and outer qualities. The characters' relationships are similarly invariable. Mickey and Goofy's personal traits are sharpened by their contrast: the witty and reflective versus the unreflective character, one imaginative and the other simple, one more heroic and one foolish, one short and the other tall, and so on. The contrast between Mickey's intellect and poet-like qualities and Goofy's simplicity is reflected in the English translation in the difference between Mickey's standard English and Goofy's colloquial accent. Goofy responds to Captain Charon, the ferryman of Hades, when the latter does not recognise Mickey Mouse: "How ignorant can yuh be, cap'n? This is Mickey Mouse! Don't yuh read comic books?" (Figure 7.3). Exceptions to expectations are again possible-Goofy, for instance, unexpectedly takes a good deal of initiative in getting through the Underworld in Mickey's Inferno; he is determined to find the exit-even though this can be explained by the exceptional circumstances. The source text of Inferno provides the story with an unusual setting, and the characters with new pseudo-identities, which create some effects of defamiliarisation, that is, limited alterations to their dominant characteristics (compare with Elseworlds stories of DC superheroes), without the need to explain these changes.

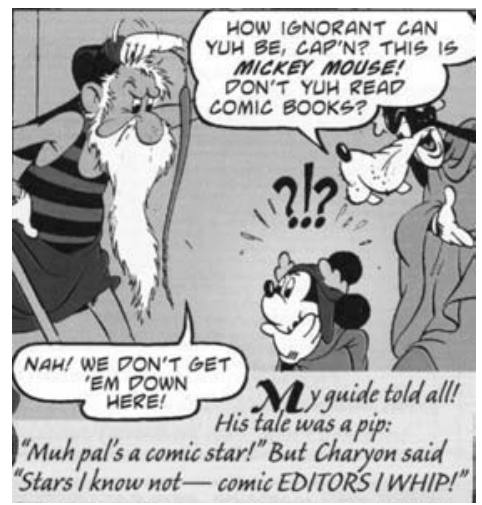

Figure 7.3 Guido Martina and Angelo Bioletto. Mickey's Inferno (1949) (C) Disney. 
Forster's categories of flat and round character do not do justice to the medium of comics, where caricature can take so many different forms and have such a variety of effects. To better understand a character's type-likeness in comics, we can turn to David Fishelov's development of Forster's model, where Fishelov distinguishes the characters' flatness and roundness on the two levels of textuality and the reader's construction. On the one hand, flatness on the textual level refers to the amount of space that is apportioned to a particular character, and the kind of literary or linguistic attention that a character is given in the text. ${ }^{19}$ The character's textual flatness typically amounts to limited and one-dimensional attention so that a character is portrayed only from one perspective, always saying the same things, repeating some pattern of behaviour, or is associated with only one trait. Examples abound in comics: Goofy's goofiness, the extreme luck of Gladstone Gander, the mayhem-seeking Dennis the Menace, the dishonest and lazy Roger the Dodger, and the unbearably malodorous Stinky in the Moomins. In contrast, in 'round attention', a character is extensively represented and referred to in the text, and characterisation may take multidimensional forms. This can involve, for instance, the presentation of consciousness and inner life, varied points of view on the character's action, behaviour, and perception-through narratorial strategies, focalisation techniques, speech and thought representation, for instance-and dramatisation in action in different situations and circumstances.

In Posy Simmonds's graphic novel Tamara Drewe (2008), which includes sustained verbal narration by various characters, two first-person narrators, Glen Larson and Beth Hardiman, are given extended and varied attention throughout the work. In their verbal narration, these characters relate the events at a writer's retreat in the English countryside through diary-style exposition. Their narration complements and contrasts with each other and with the perspectives and voices of other characters; the narrators may also quote each other or other characters. Beyond the verbal narrative track, Glen and Beth are shown engaged in action and dialogue scenes and their mental states are also related to us by means of narrative drawing. All this amounts to 'round attention' on the textual level.

On the other hand, the distinction between flatness and rotundity on the conceptual level reflects the way in which characters may be perceived in the reader's imagination as person-like entities in a fictional world. Thereby, a conceptually flat character represents some single (and sometimes simple) category, such as some moral, social, or aesthetic category. A constructionally round character is a character who cannot be portrayed in this way. Such an effect may be achieved by using multilayered modes of representation, including the presentation of inner thoughts, or complex sensory and mental processes. The crucial criterion here is that conceptual roundness requires the reader's perception 
of the character's complexity as an individual rather than as an element or the narrative structure or a theme.

Jimbo, Valise, Mickey, and Goofy are conceptually flat characters in their varying ways. The contrast between the two characters of each pair further associates them with the literary device of characterisation through contrasting types, such as Don Quixote and Sancho Panza, or indeed Dante and Virgil, where the opposites highlight each other's personality as a kind of foil. The reader's background knowledge of Jimbo's other stories, or about Mickey and Goofy's personal traits in the Disney universe, may also affect our evaluation of their conceptual flatness. Mickey's first-person narration affects the conception of this character by allowing the reader to perceive him as a more conceptually rounded character than the usual. His narration, however, does not reveal much about the narrator himself; Mickey rarely mentions his own thoughts and emotions. Instead, his narration focusses on what is happening around him and Goofy, what has just happened and, frequently, he simply quotes dialogue.

Schematic and type-like qualities can have an allegorical function. This potential is much more prominent in Jimbo's Inferno than in Mickey's Inferno. In Dante's Divine Comedy, allegory affects the whole composition, from the story events to characterisation. The narrator, Dante, is not only a poet, but he also plays the role of Everyman, or a pilgrim. His journey to Hell, Purgatory, and Heaven, during which he encounters many people whom he has known in real life, is at once a personal experience and an allegorical journey that leads him to the spiritual state of love and faith, represented by his lost love, Beatrice. At the same time, the journey reflects Everyman's fate that pertains to all people who, "Midway upon the journey of our life", start to ponder the possibility of their death and what may happen thereafter. Over the course of his travel, Dante learns the spaces that the soul may inhabit after death, but these places are also states of the soul, not real spaces. His first guide, Virgil, symbolises human reason and poetry, whereas Beatrice, his second guide, stands for love, faith, and heavenly grace.

In Guido Martina and Angelo Bioletto's Mickey's Inferno, the journey in the Underworld is represented as a kind of hypnotic dream. The allegorical level is thin or non-existent. At the end of this adaptation, however, the comic is compared to the allegorical qualities of Dante's epic. Here, it is revealed that the writer Martina and the cartoonist Bioletto's alter egos, who are tortured by Dante, who thinks that the artists have betrayed him with their adaptation, have used Mickey and Goofy's characters to gain new readers for the epic poem. It is thus suggested, by analogy, that the comic gives access to Dante's classic, perhaps similar to the way in which an allegory can illustrate complex ideas to make them more comprehensible to readers. The staging of Dante's approval of the adaptation does not make the characters of Mickey's Inferno any 
more allegorical or less parodic, however. By contrast, in Gary Panter's Jimbo's Inferno, the allegorical dimension of the story is quite explicit. Here, Hell is conceptualised as a vast shopping mall called Focky Pocky. Jimbo's joy about being once again able to look upon the stars at the end of the journey can be conceived as an allegory of Everyman liberating himself from the Hell of suburban shopping and infotainment. Jimbo may be a punk rock character by appearance and association, but he is also an allegorical figure in his own right, journeying through a consumerist Hell on earth.

One clear advantage of Fishelov's dual perspective on complexity in characters is that it allows different strategies of characterisation to result in varying effects of flatness and rotundity. Flatness on both the textual and conceptual levels creates a pure type while roundness on both levels can produce a 'pure' individual, but other combinations are equally possible. Textually one-dimensional description may also suggest conceptual roundness (a type-like individual). For instance, the trait of eccentricity in a minor character, such as Uncle Toby and Walter Shandy's obsessions in Tristram Shandy, may not be tied to a simple effect, but require complex evaluation from the reader. In these cases, an individual's single trait, such as an eccentric obsession, presupposes some psychological depth, "an element of which a mere type is deprived" (Fishelov 1990, 430). By contrast, in Glyn Dillon's graphic novel The Nao of Brown (2012), changes in colouring and visual 'flatness' add another dimension to the general round attention to the protagonist. Here, the narrator-character called Nao suffers from severe obsessive-compulsive disorder, involving especially preoccupation with violent thoughts, and often the symptoms of her mental state are also indicated by means of stylistic change. Thus, during some of her violent visions of hurting other people, the colouring of the panels may change into all red or grey, and at one point Nao Brown is also portrayed in black and white against the colour background (Figure 7.4). The uncontrollable symptoms of Nao Brown's condition are further dramatised by the embedding of her violent visions, at various instances, as parts of the unfolding events of the narrative. For this reason, the reality of her visions can sometimes be evaluated only by reading the story forward in order to detect, retrospectively, whether the violence is something that has actually occurred in the reality of her world or only consists of her inner visions.

Type-like individuals cannot be easily identified in our two Dante adaptations, but the possibility that textual flatness can have a great variety of conceptual effects is significant for characterisation in both. We might want to claim that Valise, who is given an exceptionally flat visual treatment in Jimbo's Inferno, nevertheless has some roundness in the conceptual sense as he keeps surprising the reader by the spiritual profundity of his statements. These words of wisdom include, for instance, his advice to Jimbo in canto XIX, "Don't agonize over God's divine equity", and 

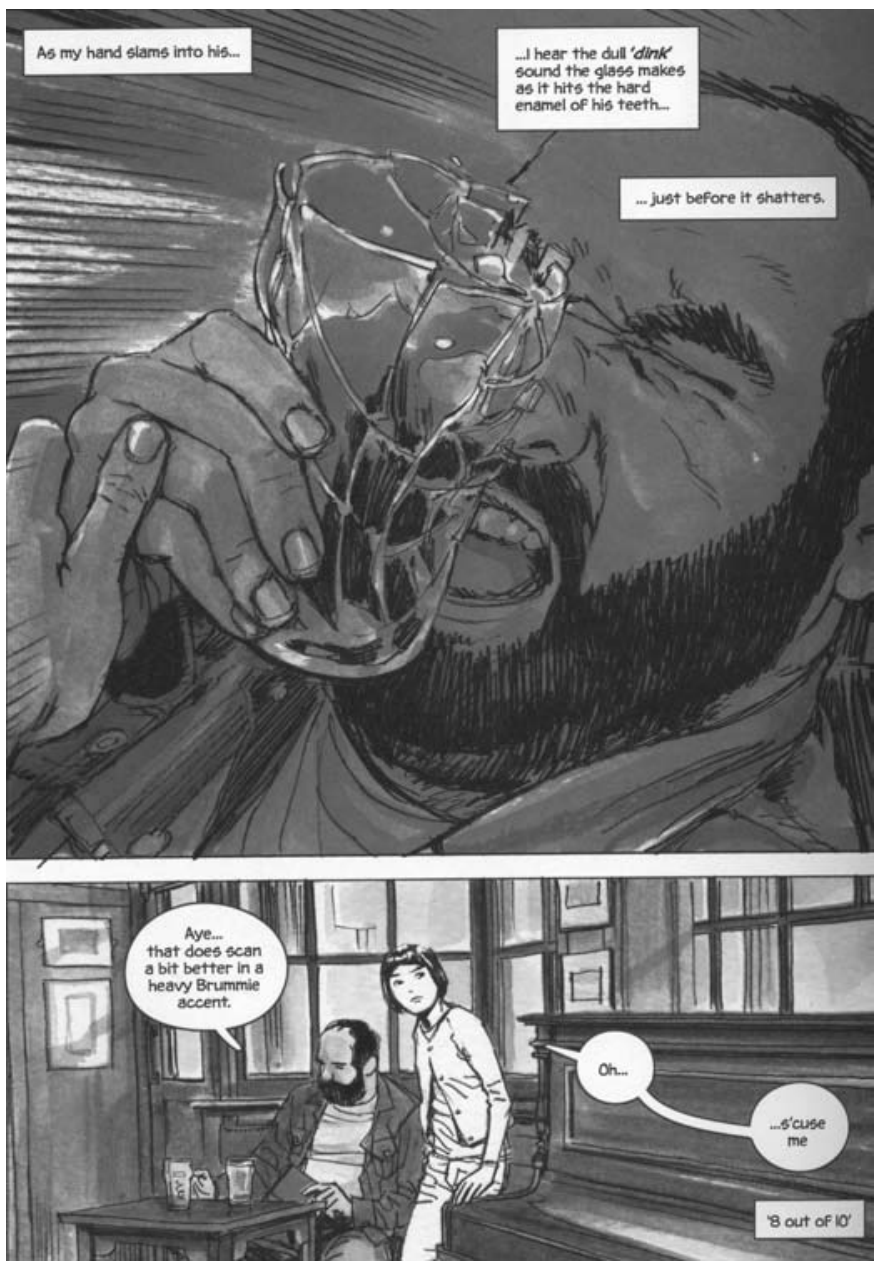

Figure 7.4 Glyn Dillon. The Nao of Brown (C) 2012 Glyn Dillon.

the insight that I quoted above concerning the burying of eggs in the sand. Whether these statements presuppose psychological depth and a sense of individuality is another matter, however. A significant challenge in ascribing mental states or psychological depth to Valise is his bluntly mechanical form. With the exception of his capacity to think and speak, and the circles on his box that could be his eyes, Valise is a radically non-anthropomorphic figure. By contrast, the appearance of Mickey and Goofy is a combination of an animal, mouse and dog-like shapes, and much that is human; their shapes serve as a kind of envelope for a flat, anthropomorphic self, but one that can display a rich repertoire of 


\section{2}

caricatured facial expressions, gestures, and postures. Mickey's character shifts towards more individuality through first-person narration, but not to the extent of becoming a type-like individual-he remains a type with some new personal features.

Individual-like types that have a round and multi-perspective representation, combined with typification on a conceptual level, are usual in comics. An individual may resemble a literary type, such as Sancho Panza; a roundly described character may also be typified thematically or socially (for instance, as a representative of ethnicity, gender, age, or any other group). In Tamara Drewe, Nicholas Hardiman and the protagonist, Tamara Drewe, are portrayed in a relatively round fashion. They are not just shown in action or dialogue in the images, for instance, but the readers also occasionally have access to their thoughts, recollections, and perceptions. They also have type-like qualities in their roles as a womanising artist, or the adulterous husband, and the femme fatale. One structurally important contrast in characterisation in this graphic novel is based on differences in terms of textual attention: the contrast between characters who are allowed to narrate at length in first-person narration (Glen Larson, Beth Hardiman, and Jody Long), and those who do not narrate, but whose thoughts and emotions are presented to us in direct discourse by other means, such as through quotations of their writings or speech and thought balloons (Tamara Drewe, Nicholas Hardiman, and Casie Shaw). The lack of continuous narrative voice does not necessarily make the latter more conceptually flat, however. On the contrary, the alternation between implicit and explicit means of characterisation may increase the interest in the characters' psychology and thus suggest new levels of complexity.

We run into problems, however, if we insist on a strict distinction between intermediate categories such as a 'type-like individual' or an 'individual-like type'. The crucial point here is that characters in comics, both within one story and with regard to one character, may create dynamic relations and combinations between type-like and individual-like qualities. Fishelov's model suggests that there is a long continuum between flatness and rotundity, or 'pure' type and 'pure' individual. The focus on such a continuum, and the variety of relations between type and individual, is especially important from the perspective of narrative comics, where types and caricature have traditionally enjoyed a central position. ${ }^{20}$ The multimodal nature of narratives in comics also often requires that we pay heed to the varying indications of flatness and rotundity, or type and individual, in words and images. Fishelov's estimate that "the modernist novel strongly tends to dispense with the 'pure' type character as well as with the individual-like type" $(1990,432)$ certainly does not hold with regard to the history of comics, even if emphasis on the complex individual may be a key element in the development of the graphic novel in recent decades. 
One advantage in Fishelov's model is that it pays due attention to the issue of the reader's active construction of characters as person-like agents. In this perspective, characters are not only products of formal devices and features of the text. However, one evident problem with Fishelov's approach is that it does not sufficiently reflect the possibility that characters serve various functions simultaneously or over the course of the narrative, as explicated in James Phelan's division into mimetic, thematic, and synthetic aspects and functions. The readers of comics may need to focus on the dynamic between these functions during the narrative progression of a story. Thereby, it must be noted that characters who can be conceived as mimetically round can, at the same time, be thematically relatively flat, and a character's textual flatness may function as a kind of guise for exploring the limits of the given type. Mimetic flatness may also give way to thematic roundness, or the foregrounding of the character's synthetic aspect may bring the thematic component into greater prominence. ${ }^{21}$ The latter is especially true in relation to the ambitious rewritings of the superhero genre in the late 1980s and the 1990s, such as Alan Moore, Dave Gibbons, and John Higgins's Watchmen (1987). Frank Miller's Batman in The Dark Night Returns (1986) maintains his superheroic label and costume (although small changes are made to the latter), but the superhero's age and pathology, as well as the dilemma of whether he could break his oath of never killing anyone, gives the type considerable new complexity and mimetic roundness. In The Dark Night Returns, Batman's allegorical role as a crusader against crime also becomes more ambiguous, as his affinity with the villains is underscored, for instance due to his lawless methods, rivalry with Superman, and split personality.

\section{On the Significance of Genre and Medium}

Generic expectations-understood as conditions of meaning and conventions of reading that relate to a specific genre-can significantly predetermine the kind of relation that the fictional world holds with the real world, including the characters' affinity to or distance from people in the actual world. Such expectations may also affect the kind of information that is given about characters. This involves, for instance, the relevance of background knowledge, the portrayal and prominence of the characters' inner life and sense of development (experimental, psychological self or signs of aging), or visual detail and verisimilitude with regard to their appearance and environment. The impact of generic expectations can be presumed to be strongest where the generic features are also the strongest, that is, in genre fictions such as humour strips, or superhero, war, romance, erotic, and horror comics that privilege particular kinds of stories, characters, and narrative modes. By contrast, a central expectation of contemporary first-person graphic novels, such 


\section{4}

as Tamara Drew or The Nao of Brown, is that they portray complex characters with considerable psychological depth.

Characters' genre-related roles, as representatives of a social type, moral categories, or themes for instance, or their relation to their perceived realism, can take precedence over real-life social knowledge, concerning, for instance, social identity such as gender, age, and ethnicity, and stereotypical frameworks, as also some empirical research has shown. ${ }^{22}$ The attention to generic expectations can also help to explain how readers set up a certain kind of character mode and fictional world, and channel their inferences according to what can be regarded as probable, possible or relevant knowledge in this world. For instance, as the readers know that characters in superhero comics have superhuman powers and capabilities, it may be expected of them to be able to throw heavy trucks off the road, shape shift and shift shapes, have incredible speed, telepathic powers, or see, smell, taste, feel, and hear more than any normal human. The readers of this genre are also likely to be aware of the fact that superheroes and supervillains are constantly recreated, revised, and adapted for new audiences. Not all comics are compatible with such expectations.

Other qualities in characterisation are medium-specific. For instance, some of the differences between literary narrative fiction and the visual means of characterisation in comics may become more evident if we think of the challenges of adaptation between these two media. Why is it that the adaptation of characters from comics into literature has been much less common than the reverse, save alone adaptations of comics into film? Comics adaptations of literature have included popular series, such Classics Illustrated or the parodies in Topolino that attempt to encourage young readers to read the source texts. In recent decades, numerous ambitious interpretations of literary classics have come out, including Martin Rowson's version of Laurence Sterne's Tristram Shandy (2010), Eric Drooker's adaptation of Allen Ginsberg's Howl: A Graphic Novel (2010), Régis Loisel's Peter Pan (1990-2004), Posy Simmonds' Gemma Bovery (1999), Winshluss' Pinocchio, or Gareth Hinds' various versions of Shakepeare's plays, just to name a few. Dante's Inferno has been adapted into graphic novels such as Seymour Chwast's Dante's Divine Comedy (2010) and Hunt Emerson and Kevin Jackson's Dante's Inferno (2012). ${ }^{23}$

The scarcity of literary adaptations of comics can be explained to some extent by different kinds of audiences, or niche markets. The power of markets is reflected, for instance, in the fact that comics are adapted into literary fiction usually only when some series becomes extremely popular. In continental Europe, some of the most popular comics series for young readers, such as the French-Italian W.I.T.C.H. and the Belgian adventure series Thorgal have been adapted into novels. ${ }^{24}$ In the United States, the main superheroes in the Marvel and DC Comics universe, 
starting with the 1940s Superman fictions, also exist in paperback series. Outside the market of adaptations, literary versions of superheroes and manga characters are quite popular in fan fiction.

Beyond market considerations, the one-sidedness of adaptations between literary narratives and comics may also be explained by the possibility in comics of overcoming certain dissimilarities between the narrative media. Comics regularly manage to tell the 'same' story as literature or, at least, make a successful parody, but the need to give up the characters' recognisable visual appearance in the novelisation of comics may seem a much bigger loss. The art of caricature, the recognisable and memorable quality of characters in comics, based on some simple visual traits, is difficult to transfer into literature. Protagonists in comics tend to have physically remarkable features, such as a sketchy face with a particular shape or expression, or head, body shape, hair, or clothing. Thus function, for instance, Tintin's quiff hairstyle and clothes-brown plus fours and brown shoes, to which are later added a white shirt, a blue sweater, and white socks-Corto Maltese's captain's hat and sideburns, the superheroes' costumes, Mickey's ears, Donald Duck's beak, and the small indications of individuality that help us to distinguish between the Smurfs.

The caricature-like and 'cartoony' presentation of characters allows comics to focus on the essential in the narrative situation at hand, such as action or the character's mental state. Scott McCloud has called the simplified reality of cartooning "amplification through simplification" (McCloud 1993, 30). ${ }^{25}$ Visual simplification and exaggeration are also means of focus. Exaggeration helps to emphasise a trait, mental state, an idea or the nature and impact of an action, for instance. In traditional Disney comics, it is usual that when characters are shocked or surprised, their hats jump in the air; a loud noise or shout can throw things around the room; and hits or blows to the head cause bumps that protrude impressively. In Mickey's Inferno, hitting and hurting are frequently accompanied by star symbols; decisiveness and persistence by puffs of smoke; shouting by drops of flying saliva; while fast movements are indicated by multiplying the same figure in the panel, and so on. Big bad Zeke Wolf also manages to run around as a mere clothed skeleton and a head when all his flesh is blasted from his body.

The demands of graphic drawing and style, the use of caricature, and the rich symbolic language of comics, easily compromise the sense of lifelikeness in characters. The use of photographic models, or the attempt to represent characters and reality in the photographic sense, ${ }^{26}$ are relatively common, but usually they occur in combination, from Hergé and Jack Kirby, Alex Raymond and Garry Gianni, to Alison Bechdel, with the cartoony techniques of simplification and exaggeration. There are many reasons why the use of photographic representation and photorealistic style is thus restricted. From the cartoonists' perspective, the systematic 
use of photorealistic style can be time-consuming and expensive. At the same time, photorealistic characterisation and reality-building has the potential to undermine idiosyncratic cartooning that draws on the cartoonist's individual style of the graphic trace-a strong expectation in the medium.

The necessity to give characters a particular visual shape clearly distances comics from literary narratives. In comics, as in picture books, external description of characters and their action can be both verbal and visual, and can either confirm or contradict each other. Psychological description can work both through words and images. Mental states may be suggested in many ways in pictures, as we have already seen, but their representation may also need, as Nikolajeva and Scott argue with regard to picture books, "the subtleties of words to capture complex emotion and motivation" $(2001,83)$. At the same time, verbal external description, especially in terms of describing the character's physical appearance and environment, becomes superfluous. Visual description is simply more efficient for this purpose, while speech as a means of characterisation is verbal by definition (Nikolajeva and Scott 2001, 83). Generally speaking, however, the visual means for depicting characters' mental states are more versatile in comics than in picture books. This is partly due to the possibilities of the sequential form. For example, a sequence of panels can depict complex mental states by contrasted perspectives, or by showing evolving emotional states in the characters' facial expressions and body language. Moreover, action and speech, or action and verbal description, are much more closely connected, both in a spatial and conceptual sense, in comics than in picture books. This is not an absolute rule, obviously, but a convention and expectation.

\section{Conclusion}

In the beginning of this chapter, I emphasised that characters do not need to be the sole focus of narrative comics, since the story's centre can also be an incident, event, plot, theme, or sometimes a space or an idea, for instance. Yet, the lack of indications of character and mental state does point to certain narrative limitations in comics. The concept of character is tied to a larger generic frame: the expectations and qualities of narrative fiction. In nonfiction, and in lyrical and abstract comics, the character category may be irrelevant, even if the representation of mental states or experience remains vital. In first-person narration in fiction, the relevance of the concept may also be limited, especially if the story is wholly focussed on the narrator-character's personal experience.

As we have seen, comics can employ a wide scope of artistic devices for ascribing mental states and personal traits to characters. Such devices include, among other things, the visual description of physical appearance, facial expressions, gesture and behaviour, speech and thought 
representation, the depiction of action, and the narrator's or other characters' descriptions. Slomith Rimmon-Kenan's emphasis that any element in literary narrative fiction, in principle, may serve as an indicator of character $(2003,36)$, that is, will further a sense of a character's personality and mental state, is perfectly applicable to comics storytelling, provided that we consider the function of images and the interaction between words and images in this process. The distinctions between a person and a thing, personal and non-personal entities, or human and (imagined) non-human experiences are crucial. We may also perceive the constant violations of these distinctions by means of caricature as another proof of the importance of that distinction. ${ }^{27}$ A character may lack cognition and sentience, at least in terms of what may be expected from humanlike characters, have a highly unnatural appearance (fantastic, invisible, or other) or, indeed, look like a thing. The character of Valise illustrates how the mere attribution of voice to an inanimate object, combined with a role in action, can turn an entity into a believable and rather complex character in comics storytelling.

The importance of external visual characterisation in this respect can hardly be overestimated. A few lines only can create characters, the art of caricature has the capacity to freely shape the characters' bodies for expressive uses, and graphic line and style offer a very efficient means for showing signs of inner life in the character's appearance, behaviour, and action. Sometimes characterisation can also be based on a contrast between visual and verbal information: a box-like robot can be philosophical; Mickey Mouse can become a medieval poet. It is then important to evaluate how much weight the reader gives to either visual and verbal narration and how much our understanding of a character's mental state, or person-like inner life, is based on verbal information. Although it may be much easier for literary fiction to develop the impression of a character's inner life-think of Marcel Proust or Robert Musil, for instance-comics can use most of the same verbal means in character description, and circumvent some of the restrictions posed by their spatial form through visual and multimodal techniques.

My choice of these examples in this chapter was deliberate in that I wanted to give more attention to the relation between type and individuality instead of focussing on graphic novels, such as Tamara Drewe and Nao of Brown, with strongly individualised characters. This was in order to better perceive how different strategies of caricature and typification may result in varying effects of flatness, rotundity, and complexity. The crucial point here is that characters in comics may suggest a great variety of dynamic relations and combinations between type-like and individual-like qualities.

A certain set of basic expectations about what characters are and how they exist are similar to comics and other narrative media, such as literature and film. Characters in comics can be expected to have inner and 
outer qualities, and they have consistency, for instance. Yet, the outer forms of the protagonists in the Dante adaptations-anthropomorphic animals, a robot, and a person seemingly without an inner self-also suggest that many aspects of human experience and embodiment may not be that relevant here. As in much fiction, characters in comics are not always processed as if they were real people, and the properties ascribed to them do not need to conform to actual world regularities (Margolin 2007, 68). In addition, graphic drawing and style, the use of caricature, and the rich symbolic language of comics set limits to lifelikeness in characters. A wide range of options exists between roughly outlined caricature and detailed photorealism in comics, but the general tendency is to allow room for caricature and the subjectivity of the graphic trace. While it is certainly possible for readers to identify with characters in comics, or find them truly convincing as persons, especially in today's graphic novels, the history of the medium is marked by a fundamental distance between real-life people and caricature. Our evaluation of the reality and complexity of human-like characters in comics, however, is also always a matter of the larger context of reception, such as generic expectations, as the rich variety of today's nonfiction comics so well illustrates.

\section{Notes}

1 Mark Blackwell defines it-narratives as "prose fictions that take inanimate objects or animals as their central characters, sometimes endowing them with a subjectivity - and thus a narrative perspective-of their own, sometimes making them merely the narrative axes around which other characters' stories spin” (2012, vii). I am thankful to the members of the ComixScholars discussion list, who responded to my inquiry about it-narratives in the spring of 2015.

2 These three works are among Groensteen's $(1999,19)$ examples of how to bypass the presence of a recurrent character in comics and still tell a story (1999, 19-20).

3 Crumb has later added other future scenarios to this work: The Fun Future, Ecological Disaster, and The Ecotopian Solution.

4 See also comics included in the anthology Comics as Poetry. Ed. Franklin Einspruch. New Modern Press, 2012.

5 David Herman has referred to the latter as models of self or personhood (2013, 134, 195).

6 Genette argues that "it seems to me that by allowing the study of characterisation to have the privilege of shaping, and thereby governing, the analysis of narrative discourse, we make too much of a concession to what is only one 'effect' among others" $(1988,136)$. The category of the character has, however, a key role in Shlomith Rimmon-Kenan and Mieke Bal's classical narratologies, and more recently, narratological theory of character has made important advances in Uri Margolin's work.

7 In this regard, the historical conception of the 'disintegration' of the classical character in the history of modern literature-a character with a proper 
name, physical and moral nature, and distinct from the narrator-and that Genette associates with Marcel Proust's Recherche (1980, 246-247), also requires us to hold onto this concept.

8 Phelan further connects the three dimensions with a scale of character functions and readerly interests in the narrative, respectively, called the mimetic, thematic, and synthetic function or interest. See Phelan $(1989,9,1996,29)$ and Phelan and Rabinowitz $(2012,113)$.

9 Henry James's much-used dictum, from “The Art of Fiction”, about the interdependence between characters and action remains relevant: "What is character but the determination of incident? What is incident but the illustration of character?"

10 Jimbo's Inferno compiles seven Jimbo comic books, published by Matt Groening in his Zongo Comics, 1995-1997, and that are reformatted to the format of the sequel, Jimbo in Purgatory.

11 Jimbo's adventures were first chronicled as a comic strip in the 1970s LA punk paper Slash and later in $R A W$ magazine.

12 Basically, any deictic linguistic marker may identify the agent of speech, thought, or action as long as the story contains sufficient information about the context of the depicted speech, thought, or action.

13 We must note, however, that some of these principles are also techniques of characterisation. What Bal calls 'accumulation' can be perceived as an effect of repetition, but also a means of characterisation. Rimmon-Kenan's 'implication', similarly, comprises various implicit means of character-building, such as revealing personal traits through action.

14 Edward Gorey explores this freedom in his "The Inanimate Tragedy", which parodies the drawn inanimate figures' supposed sentient, cognitive, emotional, and verbal qualities, and where the characters include No.37 Penpoint, The Four-Holed Button, The Glass Marble, The Two-Holed Button, The Half-Inch Thumbtack, and Needles and Pins.

15 In cross-media comparisons of this kind, it may also be forgotten that literary fiction is not always focussed on characters as individuals or on their psychology, but on types or behaviour.

16 Lack of change does not necessarily make characters flat, however (Rimmon-Kenan 2003, 41; Bal 1997, 117). Much modern literary fiction portrays, from Dickens's minor characters such as Harold Skimpole in Bleak House to James Joyce's Leopold Bloom, characters who lack development, but exhibit much depth.

17 Forster claims, for instance, that in Persuasion, Jane Austen was capable of inflating a flat character into a round character and collapsing her back into a flat in one sentence $(1953,73)$.

18 I would like to thank Mark Shackleton for this point, and also Essi Varis for her many valuable comments on this chapter.

19 Compare with Alex Woloch's notion of 'distributed attention', which refers to the attention that a specific character has in the course of the text, and character-space. For Woloch, character-space means "that particular and charged encounter between an individual human personality and a determined space and position within the narrative as a whole" $(2003,14)$.

20 Rimmon-Kenan's 'flat' pole on the axis of complexity includes characters constructed around a single trait or around one dominant trait along with a few secondary ones, such as allegorical figures, caricatures, and types (2003, 41).

21 As Phelan has shown in many of his studies on characterisation. See, for instance, Phelan $(2007,222)$. 
22 See Livingston (1998, 80-81) and Louwerse and Kuiken (2004, 171).

23 Joseph Lanzara's Dante's Inferno: The Graphic Novel (2012) utilises Gustave Dorés illustrations of the Divine Comedy to tell the story, and Robert Sikoryak's Inferno tells Dante's classic in one page as a "Bazooka Joe" in Masterpiece Comics (2009). See also Tondro (2015).

24 Hugo Pratt, the creator of Corto Maltese, has turned some of his graphic novels into prose fiction.

25 For a more historical and technical perspective on the graphic definition of character in modern comics, see Morgan (2009). Morgan emphasises, especially, the role of reproduced hand-drawn (or written) style and the swift creation, or speed-drawing, in portraying characters as types.

26 Detailed description does not always serve the effect of realism. The amount of detail in the description of Hell in Martina and Bioletto's Mickey's Inferno is impressive, but this is not realistic as such since no one knows what hell looks like. Instead, detailed description emphasises the unique qualities of this world, while it also refers to the visual imagination of Gustave Doré, and his version of that world (1861).

27 David Herman argues that the distinction between a person and a thing, or between personal and non-personal entities, is anchored in humans' embodied experience, but is also shaped by circulating cultural models of what a person is, and of how persons relate to the world at large (2013, 193-194). The division is, thus, a persisting one. 


\section{Part IV}

Speech and Thought in Narrative Comics 
This page intentionally left blank 


\section{Presenting Minds in Comics}

Narrative theory has recently shown increasing interest in the representation of consciousness in narratives. Some approaches, especially those that redefine narratives in cognitive terms, have emphasised the centrality of the mediating mind for all narrative understanding. For instance, Monika Fludernik demonstrates that narrativity, a set of properties that characterise narrative, fundamentally involves the presentation of a consciousness, reflecting on and reacting to events, and evaluating them. In developing her influential concept of narrative experientiality, Fludernik suggests that any extended narrative relies on the experiential portrayal of sequences of events and human consciousness (1996, 28-30). In the multimodal environment of comics, Alan Palmer's idea of mental functioning as action in narrative fiction (2004) provides a compelling approach by developing Fludernik's concept further. Palmer problematises what he calls the "verbal norm" in the predominant speech-category approach to narratological analysis of characters' thought processes. By the verbal norm, Palmer specifically means the preoccupation with the highly verbalised flow of self-conscious inner speech and thought $(2004,14,63-67)$, and free indirect perception where the narrator uses a character's consciousness as the perceptual angle on narration $(2004,48)$. Palmer contends that the verbal bias in narratological research has favoured modern literary fiction that employs techniques of inner speech and thought, instead of more indirect means of thought presentation, and has contributed to a limited notion of thought and mind in narrative analysis.

The analysis of mind presentation in comics storytelling might suggest ways to loosen the grip of the 'verbal norm' in narratology, while also helping us to evaluate what might work across narrative media in the speech-category approach. What makes comics, and their mediumspecific constraints and preferences, especially interesting in this respect is that the medium stimulates the viewer's engagement with the minds of characters by recourse to a wide range of verbal modes of narration in a dynamic relation with images that show minds in action. Many aspects of this multimodal interaction between words and images are similar to the way in which film narratives function, for instance in the relatively limited use of narrators in both media. Various cognitive approaches in 


\section{Speech and Thought in Narrative Comics}

film studies have also examined the audience's engagement with characters whose minds and emotions are predominantly displayed through images, with little or no direct access to the characters' verbal thoughts. ${ }^{1}$ Yet comics storytelling is also fundamentally different from film narration in this respect. ${ }^{2}$ In regard to speech-act categories, further challenges to narratological analysis of mind presentation in comics are posed by the spatial demands of the medium, which specifically limits the use of certain categories of speech and thought that are common in literary fiction. For instance, extended employment of inner speech, direct thought presentation, and thought report is relatively rare in comics. In this chapter, I shall focus on three issues pertaining to the presentation of minds in comics and graphic narratives: the mimetic aspect of the image, the problem of free indirect discourse (FID), and the interaction between visual focalisation and verbal narration in first-person narration. My theoretical discussion will be illustrated by some representative extracts from comics, moving from third-person to first-person narration, both in fiction and nonfiction. It needs to be emphasised, however, that what I refer to as first- or thirdperson narration in comics is not exactly the same as in literary fiction. The use of such grammatical persons to describe particular modes of telling is compromised by the need to base them principally on the mode of verbal telling, i.e. the continuing presence of a narrative voice, who can be identified as a character or a narratorial voice. Visual narration can always take considerable distance from the character-narrator's voice and realise a third-person framework on the visual level. At the same time, and as was illustrated in the previous chapters of this book, comics have various visual means at their disposal for subjectifying the image, regardless of the identity of the narrative voices (first- or third-person narration, or no narrator), which can also affect our understanding of the degree of subjectivity in the narrative voice. Equally, the linguistic categories of direct, indirect, and free indirect discourse, which describe the grammatical construction of speech and thought representation, need to be redefined appropriately in the multimodal environment of comics.

\section{The Mimetic Image and the Redundant Word}

Among the most obvious constraints of verbal mind construction in comics is contextual character portrayal: the rendering of a character's thoughts and sensations with the help of the physical context. The reasons for this are two-fold. The narrator's verbal report of a character's thought, feeling, and perception may be redundant with regard to the visual content and information in the images. On the other hand, reporting syntax can create an arbitrary shift in the narrative from images to words, at the risk of turning the images into illustrations of the text. These restraints are especially pertinent in the case of third-person narrative comics, but they also affect first-person varieties. 
In classical French-language third-person perspective comics and graphic novels, from Rodolphe Töpffer to Hergé, the presentation of a character's thought as mental action is realised predominantly in images, distributed through what is seen on the page, often from an impersonal viewpoint. In Töpffer's Monsieur Vieux Bois ("The Story of Mr Wooden Head", 1827), the narrator's reporting voice is quite dominant and often mentions things that can be seen in the images. Yet even here, it is the visual image that directs the reader's attention to the social situation, justifies action, and embeds a character in a physical setting. In two panels from the story (Figure 8.1), we first see the main character, Mr Wooden Head, asking for his loved one's hand in marriage at her parents' home, and then we see him in his own house, jumping for joy as his proposal has been accepted. All of his furniture seems to have been overturned. The juxtaposition of these two images, if we look only at the visual information given in the two panels, suggests an intense positive experience. The physical shape of the body, body language, facial expression, and scenery are shown rather than described. In contrast, what the images cannot fully reveal on their own is the precise meaning of the visit to his loved one's house, the difference of location (it is possible to imagine that he is jumping in the same room as in the first image), the precise meaning of his emotions while he is jumping (joy), and the duration of this jumping (3 hours).

In contrast, many features of time condensation or expansion that Dorrit Cohn associates specifically with reported speech in modern literary fiction-or what she calls 'psycho-narration', "the narrator's discourse about a character's discourse" $(1978,14)$ — can be shown in comics storytelling directly through images, without resorting to words. The different functions of summary, its iterative, durative, and

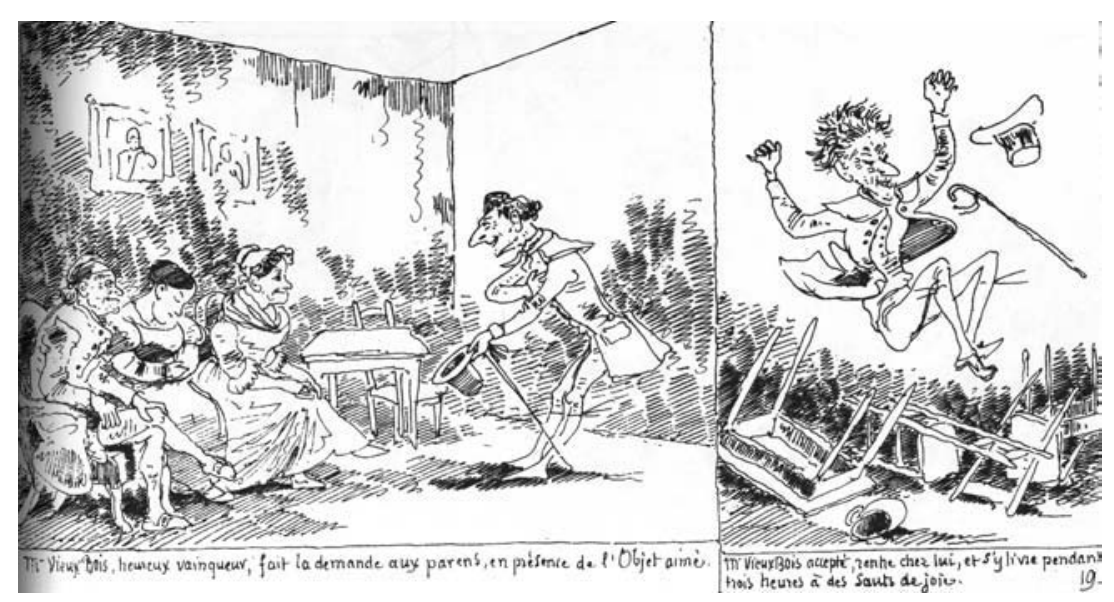

Figure 8.1 Rodolphe Töpffer. Monsieur Vieux Bois (1827). 
mutative rhythms or patterns of recurrence, persistence, and gradual change (Cohn 1978, 35), can be established through purely visual cues or through the narrative breakdown between the panel images. For instance, in an open-frame panel from Hugo Pratt's Corto Maltese. La Ballade de la mer salée (The Ballad of the Salty Sea) 1967-69, we see the repetition of the same information in a chain of changing interlocutors. The verbal content of the information-that a white young woman has been seen with a Maori man on the island-need only be related once. Similarly, the sense of the expansion of the moment, often realised in reported thought in modernist literature, can be achieved by purely visual means, for instance by enlarging the size of the picture panels or by eliminating the panel frame. Twentieth-century comics invented such visual conventions and devices to portray repetition, temporal duration, and spatial change in order to avoid the use of a sustained narrative voice. The expressive function of the panel frame's shape, size, and focalisation, pointing to changes in narrative space and time, as well as in the character's state of mind, was already part of Töpffer's narrative inventiveness. Changes in the panel size and focalisation between the images also accentuate changes in the narrative focus: the move from the polite encounter between suitor and parents in the living room to the expression of intensive private joy in one's own room. The second image brings the man closer to the reader's view, showing him from the front so that we can focus on the expression on his face.

In written narratives, the indirect discourse of reported thought and speech are often indistinguishable from scenic description. For example, when a character sees or hears something, the description of that sensory experience, introduced by verbs of perception, links his or her psyche with the scene. ${ }^{3}$ In comics, verbal scenic description is usually redundant, as images show scenes all at once, creating the effect of panoramic views, or presenting a character gazing at something and reacting to it. Verbal "telescoping" can of course serve an interpretive purpose, as a pointer to the image and its details: in Töpffer's stories, the narrator's comment actually accompanies each image. An extended use of verbal report of a character's emotions and thoughts, however, can slow down the visual narrative flow considerably. The presentation of abstract thoughts and prolonged soliloquies poses special challenges to comics in this light. Reported thought is generally restricted to localized use, as in a scene from the epic western Marshall Blueberry (Le Spectre aux balles d'or/The Ghost with the Golden Bullets) 1972, where the narrative box summarizes the character's diffuse feelings (Figure 8.2). The narrator explains that the dry basin just discovered by the character Prosit Luckner is already strangely familiar to him. The sensation of familiarity, the narrator explains, contributes to a growing sense of fear and the emergence of memories from the shady corners of his mind. 


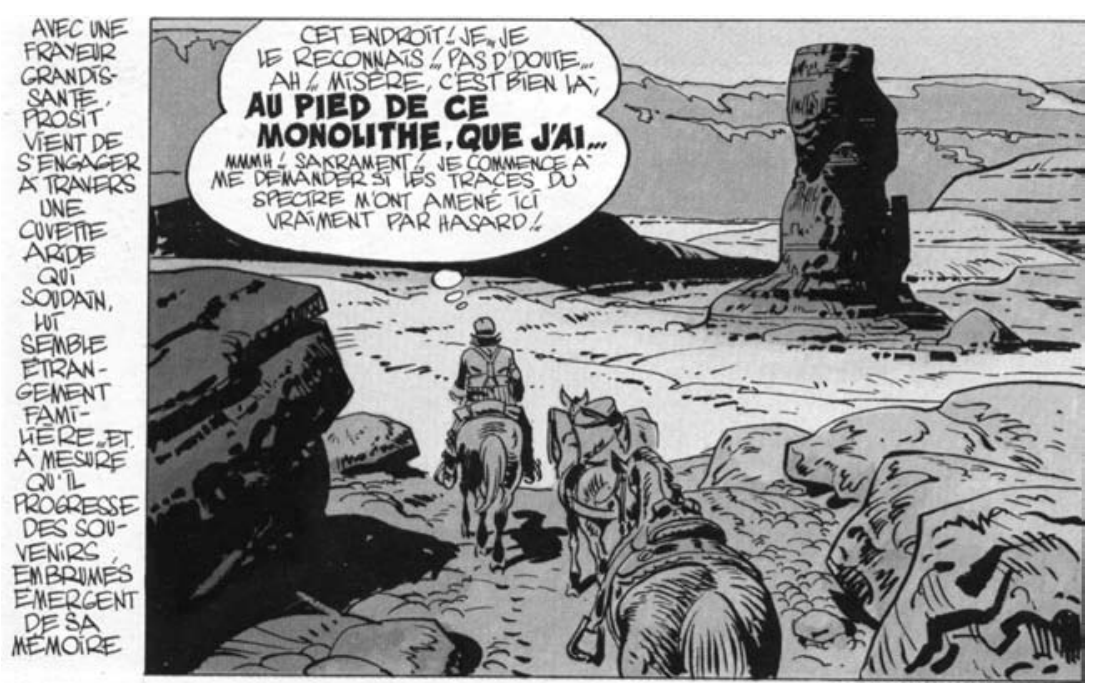

Figure 8.2 Charlier and Giraud. Blueberry: Le Spectre aux balles d'or (C) Blueberry-tome 12, Le spectre aux Balles d'or DARGAUD by Giraud and Charlier. All rights reserved www.dargaud.com.

Third-person narration that focusses on dialogue and action easily allows occasional lapses into a character's monologue, direct thoughts in thought balloons, or passages of reported thought that can have the narrative function of informing the reader about certain facts in the story (Saraceni 2003, 66-67). For instance, in Jason Lutes's Berlin (2000-2008), the shifts in visual perspective are further complicated by the way that verbal narration, in a number of localised manifestations, shifts into the direct discourse of character-narrators. Two characters, Kurt Severing and Marthe Müller, take turns as narrators, speaking as if directly through quotations from their diaries; in such passages, thought report is also used. Indirect discourse of other characters' speech and thought may also be the principal means of storytelling, for instance when this is characteristic of the personality and activity of a character-narrator, or a first-person narrative voice. In the first volume of Joann Sfar's five-volume The Rabbi's Cat (Le Chat du rabbin. 1. La Bar-Mistva, 2002), the character-narrator is a cat, whose narrating, describing, and reporting voice in the captions frames the whole story. His discourse is mostly in present tense, thus reporting what his master the rabbi, the rabbi's daughter, the rabbi's rabbi, or other people say, think, and do in the given scenes, as well as explaining his own current emotions, thoughts, and plans. The cat's initial inability to speak justifies, in a sense, the narrative situation, where indirect discourse clearly outweighs direct speech. At first, the cat can supposedly only understand 
what the humans say but cannot speak back-thus, it is logical for him to have a kind of ongoing conversation in his head-but after having eaten the rabbi's parrot, he also begins to speak. While dialogue is given more room in the subsequent albums, the cat's thought and speech report, nevertheless, remains prevalent in the story. ${ }^{4}$

In classic twentieth-century third-person comic albums, narrative comments are much more severely limited. The most common means for verbally presenting minds is dialogue, in interaction with visual information. The ironic treatment of dialogue in Tintin au Tibet (1960), for instance, where Sherpa Tharkey calls for the yeti in the mountains of Tibet and gets no answer, and Captain Haddock shouts to his own echo, presents the characters' minds in action. The scene is yet another instance of dialogue, and of action through reaction, that reveals something of the puzzling workings of the captain's mind. After Tintin has explained that what they have heard is only the echo of Haddock's voice, the captain still insists that the echo is a person with whom one could have a conversation ("The echo! No one asked him anything, the one up there!"). The interaction highlights the captain's inclination to respond to any verbal stimulus, to take every word literally, and to assume that there must always be a speaker behind every word.

The localised use of the direct discourse of inner or silent speech is relatively common in classic French-Belgian third-person examples. Unlike reported thought, which clearly belongs to the margins of the image, or to the narrative box detached from the image, inner or silent thoughts can be placed within the picture panel with visual markers of its own, mainly by the thought balloon. Most typically, in the Western tradition of comics, the thought balloon is connected to the character by a line of smaller bubbles, instead of the arrow-like tail common in speech balloons. The thought balloon forges a double link between the text and the image in that it simultaneously constitutes a message while its presence, position, and visual shape also convey information relating to the character's state of mind. The example above from Blueberry involves an instance of inner speech, indicated by the thought balloon. In the balloon, we hear Prosit's direct reaction to what he sees. The bold letters of the sentence "au pied de ce monolithe, que j'ai..." ("at the foot of this monolith that I...") indicate the most important and emotionally charged content of these thoughts, the recollection of what the character has done at the foot of the monolith. The possibility of integrating inner speech and thought within the graphic image, while indicating the emphasis or other qualities of the thoughts, makes this speech category convenient for the medium. Any extended use of this mode, however, naturally alters the balance between the visual and the verbal components in favour of the latter.

Thus, third-person behaviourist comics like Tintin and Corto Maltese, a genre still predominant in the medium, very rarely explore characters' 
psyche through indirect verbal discourse like reported thought. ${ }^{5}$ Instead, these narratives resort to direct verbal discourse through dialogue and inner speech, as well as multimodal and visual narrative techniques, which we have discussed earlier in this book (graphic showing, focalisation).

\section{Free Indirect Discourse in Comics}

To examine some of the medium-specific features and limitations of indirect speech and thought in comics, I would like to turn to two vastly different adaptations of Gustave Flaubert's novel, Madame Bovary. Posy Simmonds' graphic novel Gemma Bovery (1999) is a self-reflective rewriting of the novel set in our contemporary world, and it follows Flaubert's story only partially. Bardet and Janvier's adaptation in the album format Madame Bovary (2008), in contrast, faithfully quotes Flaubert's language and closely follows the order of the events in the novel. ${ }^{6}$ My focus in this comparison will be on the literary style of free indirect discourse (FID), and the accompanying technique of shifting viewpoints (or focalisation) between internal and external positions, for which Flaubert's novel is so well known. FID entails, without being clearly internal or external, both a reference to the perceiving subject (the character) and the narrating instance (the narrator).

Before moving onto a more detailed textual analysis of the examples, however, we must examine the difficulty of transferring FID to visual storytelling and multimodal texts, where the scope of the verbal element is limited and combined with other kinds of signs. Analysing the major film versions of Madame Bovary, Mary Donaldson-Evans underscores the difficulty in transferring FID to the visual medium:

Whereas the shifting narrative viewpoint created by Flaubert's use of this narrative style can be easily transferred to the screen, the camera naturally embracing the perspective of different characters, the simultaneous representation of two viewpoints and the ambiguity and irony that often result are difficult to convey in film, where differing perspectives must be represented sequentially.

(2009, 31-32)

The only exception that Donaldson-Evans can think to this rule of non-simultaneity of different perspectives in films is "the use of a voiceover narration that mocks the picture on the screen" $(2009,32, \mathrm{n} 3)$. While the ambiguity that can result from the use of FID in literature is hard to convey in film, the claim about perspective-taking in cinema seems to rest on questionable premises.

First, it is not clear how literal simultaneity in viewpoints could be achieved in the temporally organised order of literary narratives. Changes in the windows of focalisation in Flaubert's Madame Bovary 
are presented sequentially, as the narrative moves from Charles to Emma and back (or between other characters and the extradiegetic narrator). FID can maintain, by linguistic means, the third-person reference while reproducing the character's own language. This relation of voices may remain ambiguous as to the source of the words or, equally, constitute a clear hierarchy where the narrator cites some character's words without quotation marks. Still, to equate the relationship between the narratorial context and a character's voice with 'perspective' or 'simultaneity' in a literal sense is quite another matter. The various theories of 'embedded focalisation' or 'dual focalisation' that have been proposed in literary narratology usually try to come to terms with the temporal ambiguity of this concept. ${ }^{7}$ Donaldson-Evans seems to uncritically collapse voice and focalisation, perhaps misled by the fact that in literature, our interpretation of these elements is based on the same linguistic features in the text. The separation between these two structures of narration remains important in novels where the perspective, which is usually closely tied to the characters, keeps shifting and where the narrator has varying degrees of presence.

Second, there are other pertinent disparities between media. In visual storytelling, and in the spatial arrangement of comics in particular, several focalisers can appear simultaneously. For instance, fields of vision in a panel can include a sense of a subjective vantage point without being limited to it, thus violating clear boundaries between external and internal perspectives. In Bardet and Janvier's version of Madame Bovary, the close-up of Charles's eyes, as he waits for a sign as to whether Emma wants to marry him, brings us close to Charles's vision (Figure 8.3). The subsequently represented field of vision then embraces the character's viewpoint. The illusion of subjectivity in this sequence is based both on the juxtaposition of the perspectives in the first image-the focus on the man looking and his gaze, which is directed at something that we do not see-and on the shift in perspective between the panels.

In fact, many common techniques of embedded point of view in films, including ones that are also commonly used in comics, have been likened to FID. For instance, Charles Forceville has suggested that studying cinematic techniques like character-bound camera movement in terms of FID, specifically when such techniques create ambiguity between the external "narrator" and the character, could contribute to a transmedial narratology $(2002,133)$. Another use of embedded viewpoints is the over-theshoulder shot, in which the external focaliser in a narrative watches "along with a person", but without leaving focalisation entirely to this character (Bal 1997, 159). The technique, as Mieke Bal has suggested, is comparable to FID, in which the narrative discourse approximates a character's voice without letting him or her speak directly $(1997,159)$. I believe these to be interesting suggestions for the study of comics and visual narratives in general, as long as we are ready to modify our understanding and 


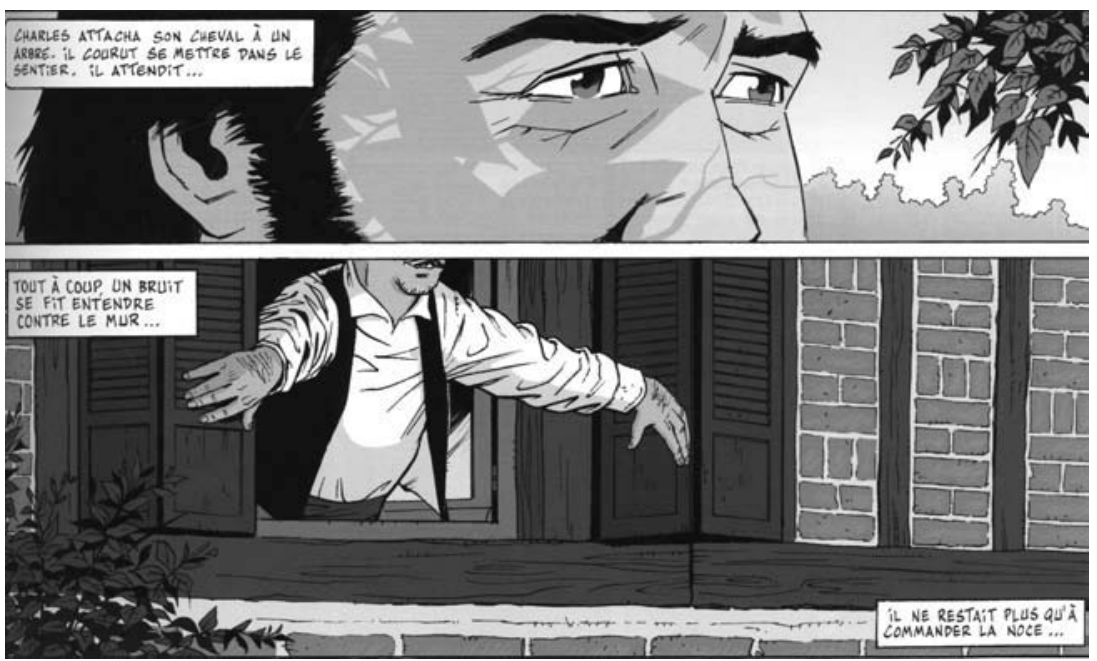

Figure 8.3 Madame Bovary par Daniel Bardet et Michel Janvier (C) Éditions Adonis, 2008.

use of speech-act categories in the multimodal and mixed-media environments. However, these cases chiefly represent forms of visual ambiguity even if they may filter a sense of subjective perception and cognition, and not ambiguity in the sense of speech and thought.

All these (and many other techniques) can accommodate the effects of FID on the visual plane as they combine a character's viewpoint with some more objective perspective or manifest one character's thoughts inside another's. Visual narratives have, in fact, so many means at their disposal for creating embedded perspectives that they are vital, in adaptation, to compensate for the limitations the narratives face in the representation of thoughts by visual means. To better see where the medium-specific constraints lie in this respect, it might help us to distinguish between free indirect speech or thought and free indirect perception. In literature, free indirect perception, sometimes also called represented perception, means the presentation of a character's non-verbalised perceptions as they occur in his or her verbalised consciousness (Prince 2003, 35). Visual narratives, by contrast, are characterised by their capacity to show fields of vision as if they were someone's non-verbalised perceptions. However, at the same time, it is challenging to describe the subjective meanings of these perceptions by visual means alone. Thus, the difficulty in transferring novelistic techniques of thought representation to comics does not lie in the simultaneity of the viewpoints - which may, in fact, be more viable in the spatial arrangements of visual narratives than in literary discourse-but the way that 


\section{Speech and Thought in Narrative Comics}

certain techniques of indirect speech and thought, such as FID in literature, can give the reader access to some subjective psychic reality and mediate that access through narratorial sympathy or irony. In Flaubert's Madame Bovary, FID typically reflects a subjective mind onto the objective structure of the language of narration, emphasising expressions of emotion or the irony in the mind's illusion.

Take the passages towards the end of the first part of Flaubert's novel, when Emma is growing more and more dissatisfied with her country doctor husband and their life in the commune of Tostes. Here, her thoughts and emotions are given in alternating stretches of reported thought and FID, while the perspective is mediated through internal focalisation:

Emma le regardait en haussant les épaules. Que n'avait-elle, au moins, pour mari un de ces hommes d'ardeurs taciturnes qui travaillent la nuit dans les livres, et portent enfin, à soixante ans, quand vient l'âge des rhumatismes, une brochette de croix, sur leur habit noir, mal fait. Elle aurait voulu que ce nom de Bovary, qui était le sien, fût illustre, le voir étalé chez les libraires, répété dans les journaux, connu par toute la France. Mais Charles n'avait point d'ambition!

(Flaubert 1993, 76)

Emma looked at him and shrugged her shoulders. Why, at least, was not her husband one of those men of taciturn passions who work at their books all night, and at last, when about sixty, the age of rheumatism sets in, wear a string of orders on their ill-fitting black coat? She could have wished this name of Bovary, which was hers, had been illustrious, to see it displayed at the booksellers', repeated in the newspapers, known to all France. But Charles had no ambition. (Trans. Eleanor Marx-Aveling, Flaubert 1946, 69-70)

The viewpoint is clearly Emma's. At the end of the passage, further, the exclamation point functions as an emotion marker, suggesting a shift from reported thought to free indirect thought. Some telling transmutations to the narrative mode occur in Bardet and Janvier's adaptation of this scene. The line "Mais Charles n'avait point d'ambition..." ("But Charles had no ambition..."), now accompanied with an ellipsis, becomes part of the narrator's thought report. A few panels later, another passage in FID, this time in Charles's perspective, is shortened and transmuted into a brief dialogue between Emma and Charles:

Il en coûtait à Charles d'abandonner Tostes après quatre ans de séjour et au moment où il commençait à s'y poser. S'il le fallait, cependant! Il la conduisit à Rouen voir son ancien maître. C'était une maladie nerveuse: on devait la changer d'air.

(Flaubert 1993, 78) 
It cost Charles much to give up Tostes after living there four years and "when he was beginning to get on there." Yet if it must be! He took her to Rouen to see his old master. It was a nervous complaint: change of air was needed.

(Trans. Eleanor Marx-Aveling, Flaubert 1946, 69-70)

The predominant narrative mode in Flaubert's novel is what F.K. Stanzel has called a 'figural narrative situation', ${ }^{8}$ centred around the main reflector-characters, Emma and Charles. This entails that the readers are allowed to access their inner world, but this access is mediated by the narrator, as indicated by the fact that the characters' reflections are largely cast in language that they would not use. There are only brief instances of direct thought where the main characters articulate their thoughts.

As the transmutations above suggest, it is difficult to find equivalents in comics storytelling for narration that severely restricts the use of direct discourse, such as dialogue or inner speech and thought, and prefers more indirect forms of speech and thought representation. Bardet and Janvier's version of Madame Bovary simply dispenses with the free indirect passages even if, in other respects, their version strives to be as loyal to Flaubert's language as possible. The outcome is a neat distinction between the direct discourse of the speech and thought balloons on the one hand and the indirect narratorial discourse of the narrative boxes on the other hand. There are only a few brief exceptions to this arrangement. ${ }^{9}$

Bardet and Janvier's adaptation radically reduces the variety of techniques of representation of thought and speech in Flaubert's novel. This does not concern only FID, but all more complex cases of direct discourse or shifts between different modes for speech and thought representation as well. To take another example, we may think of the scene in which Rodolphe writes a letter to Emma to dismiss her. In the novel, the scene is narrated through a complex alternation between indirect thought report, FID of the writer's reflective consciousness, and direct discourse, including Rodolphe's speech to himself and thoughts or quotations from the letter that he is writing. In the comic book version of the same passage, there are quotations from the letter in the panels and some brief thoughts given in direct discourse and thought report. The narration thus becomes much more straightforward, as if underlining the importance of the story (order of the events) over the discourse (order of presentation) in adaptation. Certain visual elements in this scene, nevertheless, manipulate the distinction between internal and external perspectives in a way that may remind us of the effects of FID. In particular, the incorporation of Rodolphe's handwriting and the showing of his hands in the foreground subjectify the perspective, although they do not render it fully subjective. 


\section{Speech and Thought in Narrative Comics}

In contrast, Posy Simmonds' adaptation of Madame Bovary as Gemma Bovery takes advantage of a great variety of types in thought and speech representation. A crucial structural element that makes this variety possible is the employment of a consistent first-person narrator, who is a witness to the main events of the story, while his personal perceptions are at the core of the narration. With regard to the technique of FID, however, both of these versions avoid it. In Simmonds' Gemma Bovery, the main verbal mode in thought representation is the narratorial report of Gemma's diary, which is interrupted by frequent direct quotations from her writing or the narrator's reflections and observations. What is quite different from Bardet and Janvier's Bovary is the use of a homodiegetic witness-narrator, accompanied by the 'objective' focalisation in the images, which allows smooth perspectival changes, and changing degrees of irony between the narrator, called Raymond Joubert, and the other characters. Despite the focus on Gemma's adventures-her marriage, exile, and adultery-Joubert's personal experiences, perceptions, and evaluations of Gemma's behaviour remain at the core of the story, creating a continuous "voice" that frames the story and the quotations. What adds to the irony is that the narrator is obsessed with what he sees as coincidences in his British neighbour Gemma Bovery's life and that of her near namesake, Emma Bovary. Thus, irony, unlike in Flaubert, extends to the narrator himself, particularly with regard to amorous Joubert's spying and infrequent obtrusiveness, such as when he sends, out of jealousy or due to his delusions of coincidence between Emma's and Gemma's life, anonymous letters to Gemma and her friends in order to influence their behaviour.

The narrator's comments, interpretations, and summaries in direct discourse, concerning the events or Gemma's diary, make an exception to the general divide between direct and indirect discourses on the verbal plane. These commentaries are marked off from the main body of the narration by decreased font size. Other devices for marking more direct speech or thought within indirect discourse is the inclusion of Gemma's writing in the images, or the use of French in quoted dialogue in the images, with the accompanying English translation. The question of interlinguistic transaction becomes also occasionally evident in Joubert's difficulty with colloquial English expressions that he cannot find in his dictionary. An even more important means of manipulating the relation between direct and indirect discourses in this narrative, however, is the visual perspective and visual showing that give the reader information beyond what could be included in any written diary, both in terms of visual details and people's thoughts, speech, and dreams. The images may, for instance, cast the narrator in an ironic light. 


\section{Interaction of Verbal and Visual Narration in First-Person Comics}

With regard to first-person narration in literary fiction, the roles of the narrator and the character may diverge and become more independent from each other, for instance when the character-narrator tells about things that he has not witnessed or seen. Gerard Genette has dubbed this technique, where the narrator gives more information than is authorised by the overarching form, paralepsis $(1980,195)$. Similarly, in third-person figural narration, as happens with the narrator's rhetorical questions in Flaubert's Madame Bovary, or in some descriptive passages in the novel, the narrative may deviate from the main characters' represented perceptions to a clearly external and nearly omniscient perspective.

Comics storytelling may take advantage of a similar alteration in narrative mode, while it can rely on this disparity in a much more systematic way: the voice is subjective, but the perspective in the images shifts between various more or less objective and subjective positions. The divergence between the verbal narration of Joubert's consciousness and the perspectives in the images suggests varying degrees of distance between telling and showing, the narrator's voice, and his embodied existence in the fictional world. The complex narrative situation in Simmonds' graphic novel, and one that is somewhat unusual for the medium, achieves similar effects to that of FID in Flaubert's Madame Bovary, making the role and significance of the narrators' involvement and moral stance central to the story. Other examples of the difficulty in pinpointing the exact degree of subjectivity in the images in Simmonds' Gemma Bovery are the documents that are included in the book, such as menus, restaurant reviews, letters, notes, advertisements, magazine articles, or objects mentioned in Gemma's diary. To whom can we attribute these images? Are they part of the narrator's direct discourse or paratexts that are neither direct nor indirect? Are they shown as they were seen by the characters? Or does it really matter?

Alan Palmer $(2004,15)$ defines a 'continuing-consciousness frame' as the reader's creation of a sense of consciousness out of the isolated passages of the text that relate to a particular character, and 'thought-action continuum' as the idea that action and consciousness descriptions are often inseparable in fictional narratives. These are useful tools for analysing homodiegetic graphic narratives where the narrator is a character in the story and where the narrator's voice is sustained throughout the story. For instance, in Guy Delisle's travel memoir from North Korea, Pyongyang: A Journey in North Korea, the narrator's words provoke a sense of a continuous 'voice-over' in the reader's mind. Tensions between verbal narration, scenic showing, and visual focalisation are crucial to understanding the story. The narrative employs various means of visual focalisation from the subjective perspective to the objective camera eye. The story begins with the narrating 'I's arrival at a North Korean 
airport where no electric light can be used. The scene is first shown from an impersonal, exterior perspective. It soon becomes evident, however, that the viewpoint actually belongs to the passenger going through customs, the narrator himself. The subjective viewpoint is finally evoked in the panel where the eyeline is matched with the character, who is looking at the shadowy man who greets him. The subjective perspective is then affirmed gradually through several changes in the windows of focalisation, from the external showing of the scene to the main character's angle of vision and finally to his verbal self-communion.

Another aspect that is significant in this passage, and typical of many first-person comics today, is the presence of two first persons: the narrating voice and the experiencing self who is seen. The voice and the view of the narrating 'I' can coalesce in one panel, but they can then again be severed: the narrator re-emerges in the following panels as a figure seen from the outside. The transition is gradual so that we are first shown the narrator's shadow emerging from the side of the panel's frame, as if in between the interior and the exterior of the panel, whereas the next panel shows him from an external viewpoint. It is important to note that narration in Pyongyang builds on transitions of this sort between the narrative of the mind and the showing of the body, and the act of perception, which is itself split between the perspectives of the experiencing and the narrating self. A whole range of effects is produced by these transitions.

By contrast, in Paul Hornschemeier's autobiographical fiction Mother, Come Home (2003), there is an unusually sustained use of reported thought, related to two specific features of the story: it helps to create the temporal perspective of self-narration - the narrator's investigation of his childhood experience- and it contributes to the narrator's concern about the state of another person's mind. Self-narration here manipulates not only the distance between the narrating, verbal 'I' and the experiencing self of the visual figure, but also the disparity between the narrator's present self and his childhood self. The narrative presents the narrator's childhood self as if to assess it. The story of the death of the narrator's mother and his father's subsequent deep depression, eventually leading to suicide, is told through a perspective many years in the future. Reported thought is employed frequently when the narrator tries to understand the diffuse thoughts and feelings that he had at the age of seven, and the decisions he made at that time. For instance, the narrator explains that one long walk to the graveyard, which we see in the images, marked an important turn in the boy's understanding and experience. In his summary of these memories, the narrator also elaborates on the cognitive and linguistic disparity between the narrating adult and the child. On several occasions he points to the fact that as a boy he did not understand what was happening around him, especially in the mind of his father. The child's alienation, and his cognitive and temporal distance from the narrating 'I', is further visually dramatised by the lion mask that is often drawn on his face. 


\section{Conclusion}

What does this survey of the strategies for presenting speech and thought in comics reveal about the specific constraints and options for presenting minds in this medium?

It shows that graphic narration subjects to doubt certain theoretical presuppositions prevalent in much narrative theory that is based on literary narrative fiction, and requires us to critically examine and redefine various key distinctions, such as those between first- and third-person narration, between direct, indirect, and free indirect discourses, and between telling and showing. In a multimodal narrative comic, vast areas of mind presentation are not suitable for analysis using the concepts of narrator and voice or the speech-category approach. The question of narratorial authority, enunciation, and control-Who tells the tale? Who authorizes narrative meaning? Who controls it?-must also be posed differently when speech categories interact with graphic images.

In reading and viewing visual narratives, we often see the mind in action from a focalised perspective or through a figure in action. However, it is often doubtful whether third-person comics involve a sustained continuing-consciousness frame in the same sense as literary heterodiegetic narration pertaining, for instance, to varieties of "omniscient narrator". The framing and the focalisation of the panel image typically bear traces of personal or impersonal intelligence, but the attribution of these traces to a continuum of a single individual consciousness is often impossible.

Understanding the workings of the characters' and the narrator's minds is important in most graphic novels and nonfiction. But typically, comics homogenise their visual compositional techniques and sensibilities to create a sense of a coherent narrative whole or to foreground elements of a persistent graphic style. With so-called behaviourist narratives, the reader can also always construct a sense of consciousness from dialogue or mere descriptions of behaviour when there are no inside views. What helps in this construction is that impersonal points of view, though revealing things that the character could not see, are often related to, or encompass, or are synchronised with, the character's field of vision. They show scenes as if from behind the character's back or by his or her side, present him or her looking at something, or include his or her point of view within a broader field of vision in other ways.

The examples that I have discussed above all portray characters in action within social environments. The showing of the characters and their actions from panel to panel creates a sense of a continuing tale, and establishes what Palmer calls a characters' 'frame'. ${ }^{10}$ Keeping in mind the major role of the authorial focalisation of the impersonal viewpoint ('camera eye') in all comics storytelling, we may conclude that the sense of a continuing subjective perspective is not a necessary semiotic channel for mediating information in such narratives. As the ongoing debate over the notion of 


\section{Speech and Thought in Narrative Comics}

a cinematic narrator has proved, it is not as simple to argue about visual narratives-where the presence of the narrative agent is only implicit-as it may be about heterodiegetic literary narratives, that the viewers necessarily attribute stylistic features to a hypothetical narrator persona. ${ }^{11} \mathrm{Be}-$ haviourist and first-person comics, likewise, constantly exploit the various possibilities of external, objective viewpoints. While developing what she calls "visual narratology", Mieke Bal has admitted that even if the distinction between external and internal focalisers may hold in visual arts, this distinction is not always easy to point out $(1997,163)$. In her comments on the American artist Ken Aptekar's paintings, Bal refers to a similar and potentially ambiguous relation that I have discussed above, between the "external" positioning of the figures who are shown in the image and the direction of their gaze, hands, or other gestures that may call our attention to specific aspects within the space of the image. Other types of challenging contrasts and fusions of internal and external perspectives in comics storytelling include the function of the panel frame as a potentially identifiable frame of vision. Within a sequence of images, the panel frame can easily alternate between indicating either external or internal vision, or narratorial or figural (character-bound) focalisation. Furthermore, the images and words in a panel can point to events that take place outside the frame and are, therefore, only indirectly linked with what is seen.

Study of the cognitive construction of consciousness, especially with regard to behaviourist fictional narrative and contextual character portrayal, can be helpful in understanding the presentation of minds in comics. Recent narratological work on fictional minds is important in pointing out the often unjustified privileging of 'intrinsic' character portrayal in classical narratological approaches, based on modernist figural literary narratives of inner speech, at the expense of reported speech and thought or the showing of a mind in action. Nevertheless, third-person graphic novels differ from third-person realist and modernist novels, which are Palmer's main focus in Fictional Minds and Social Minds in the Novel (2010), in that both inner speech and reported thought usually serve only limited, local purposes. At the same time, the meaning of a 'behaviourist', 'neutral', or 'objective' narrative may have to be rethought in this medium-specific context where the reader's cognitive ability to read minds is tested not only in words and images but also in placing the two components in interaction. Transitions between internal and external visual focalisation are often essential in the understanding of the story, while shifts between direct and indirect verbal modes of narration are not as smooth as they may be in literary narratives. Inner or silent speech can be visually marked, integrated within the space of the image in thought balloons. Indirect speech, in contrast, such as the reported thought of a third-person consciousness, remains doubly indirect, difficult to place within the image and always in danger of repeating the information given in the image. In the multimodal context 
of comics storytelling, the directness of speech or thought can only be adequately evaluated against the visual information of the image, just as the personality or impersonality of the viewpoint in the image must be assessed against the verbal content. I hope that it has become evident that the study of mind construction in comics needs to go beyond the speech-category approach, and beyond the categories of narrative situation and focalisation that are based on literary examples, to consider speech acts in interaction with visual information and narration by showing. Moreover, the study of mind construction in comics storytelling can benefit from examining the act of showing as a form of thinking, and caricature (of face, gesture, pose) as a form of perception.

\section{Notes}

1 See, for instance, M. Smith (1995), G. Smith (2003), Bordwell (1985), and Branigan (1992).

2 On the formal relationship between comics and film, see Boillat (2010), Lefèvre (2011), and Kolp (1992).

3 See Cohn $(1978,49)$.

4 Saraceni, while helpfully discussing the presentation of speech and thought in comics through linguistic categories, mistakenly claims that speech and thought can only be reported directly in comics $(2003,62,69)$.

5 Gerald Prince defines a behaviourist narrative as a "narrative characterized by external focalisation and thus limited to the conveyance of the characters' behavior (words and actions but not thought or feelings), their appearance, and the setting against which they come to the fore" $(2003,10)$.

6 Bardet and Janvier's version belongs to the Romans de Toujours series, which is marketed with the intention of increasing interest in "treasures of world literature" among young readers. To further this purpose, Bardet and Janvier's Madame Bovary also includes a CD version of the novel and a dossier that gives relevant information on the writer and the social, economical, and political situations in France 1815-1848.

7 See, for instance, Phelan (2005, 118-119).

8 In this type of narrative mediacy, a reflecting character "thinks, feels and perceives, but does not speak to the reader like a narrator. The reader looks at the other characters of the narrative through the eyes of this reflector-character. Since nobody 'narrates' in this case, the presentation seems to be direct" (Stanzel 1984, 5).

9 These include, for instance, the moment when Emma considers leaping out of the attic window after she has received Rodolphe's letter of dismissal. Here, the first two sentences, "Pourquoi n'en pas finir? Qui la retenait donc?" preserve the sense of free indirect style, placing Emma's thoughts within the narrator's discourse.

10 The reader's processing of fictional minds based on bidirectional information flows, such as hearing about the characters for the first time or setting up initial hypotheses about them (top-down information), and being fed new information about the character in the text and thus modifying earlier information about them (bottom-up information) $(2004,176)$.

11 Fludernik argues that in terms of reader response to individual literary texts, "the tendency to attribute stylistic features to a hypothetical narrator persona and/or a character is a simple fact" $(2001,622)$. 


\section{Dialogue in Comics \\ Medium-Specific Features and Basic Narrative Functions}

Conversation is a basic element in the medium of comics, where much of the narrative appeal is derived from the interplay between dialogue and action. The speech balloon, a favoured visual symbol for voice and utterance in the medium since the mid-twentieth century, has become a symbol for comics. In Italian, famously, the word fumetto- the word for a speech or thought balloon-also refers to the art form itself, whether in the form of a comic strip or a comic book. In fact, dialogue is such a central feature in the medium that it may sometimes be difficult to think of it as a distinct element. A character who speaks his thoughts aloud when apparently nobody is listening is a much-used convention, and many comics, for instance, 'talking heads' or humoristic comic strips that deliver a verbal gag, focus on speaking. Perhaps paradoxically, dialogue scenes may be more distinguishable when their use is more restricted, for instance, in comics when action is predominant and only occasionally interrupted by a scene of talk or when first-person verbal narration is predominant, as in autobiographical comics that occasionally lapse into dialogue.

The reason for the popularity of the dialogue form in comics is at least partly related to medium-specific constraints and affordances that encourage its use and, concomitantly, restrict the employment of more indirect forms of speech and thought representation. In contrast with dialogue, forms of indirect discourse, such as free indirect discourse or the narratorial reporting of a character's speech, tend to demand more space for words. Conventional strategies for distinguishing between these modes of verbal narration have included their visual form and placement in relation to the images. The dichotomy between narratorial voice in caption boxes and dialogue or other forms of direct speech in text balloons is not always clear-cut, let alone all-inclusive. Speech in comics can also occur in captions, verbal narration can take place in text balloons, the narrator's and the character's voices may intermingle, ${ }^{1}$ and neither verbal narration nor direct speech or thought must be placed in boxes or balloons. Moreover, text in comics can occur outside these two categories in the image background or as part of the image. However, the continued assertion of the difference between direct speech and 
other modes of verbal narration in comics also needs to be taken into consideration as an important convention in the medium.

This chapter focusses on the dialogue form as a key narrative device and technique, and it examines the main compositional principles and narrative functions that characterize conversational scenes in comics. The starting point in this investigation is the multimodal character of speech and conversational exchange in comics. This requires us to focus on the interaction between the utterance and the elements of the image. Thus, on the one hand, I will discuss the ways in which dialogue, in the form of written speech, interacts with what is shown in the image, such as the interlocutors' facial expressions, gestures, body language, and other visual cues of mental states and participant involvement. Furthermore, this necessitates an investigation of the visual possibilities and expressive functions of typography, the graphic style of writing, onomatopoeia or imitatives, ${ }^{2}$ visual symbols, and standalone non-letter marks in the written rendering of conversation. On the other hand, I will discuss the function of speech balloons as metaphors for an utterance-'utterance' meaning here a specific piece of dialogue-voice, and turn-taking, and their narrative role in organising the time of the speech event and the order of its reading. Utterances in comics are characterised by their dual role as both instances of imagined speech in the world of the story and written language to be read. As to their latter function, it must be taken into account that readers of comics need to process the relations between the various utterances both in a single panel, when it includes several utterances, and between the panels in order to create a sense of a continuous conversation. Finally, I will briefly discuss some strategic uses of contrast and emphasis between visual and verbal narration in speech representation in comics.

The ultimate goal of this chapter is to develop a medium-specific understanding of the dialogue form in comics and outline the basic narrative functions of scenes of talk in comics. In this investigation, different examples will be drawn from innovative uses of dialogue in this medium. The subject is admittedly very broad. Within the bounds of this chapter, I can merely hope to highlight the main features of interest in this crucial and often central form in comics.

\section{The Embodied Speech Situation in Comics}

Given the multimodal nature of the medium and the importance of visual showing in comics, the question of dialogue in comics requires us to think of the areas of interaction between the image content, such as the portrayal of the participants in the conversational scene, the utterance that is placed in the image, and the main formal aspects of the composition, such as panel relations and the page layout. First, let us consider the ways in which the participants in such scenes are visually shown to be engaged in 


\section{Speech and Thought in Narrative Comics}

the speech situation by means of non-verbal communication. Such means include, especially, facial expression, posture, hand gesture, eye contact (or gaze), and the expressive distortion of the interlocutors' bodies.

Existing research on the gesture-utterance connection in comics suggests that the use of gestures as signs of emotion largely follows real-life models in everyday speech situations (Fein and Kasher 1996; Forceville 2005). Both in everyday real-life conversation and comics, body language and posture are elemental communicative resources. At the same time, however, research has also suggested that in comics, since they commonly simplify and exaggerate bodily forms through caricature, the speaker's and the recipient's gestures often have a more prominent role than in real life (see Forceville 2005, 85; Fein and Kasher 1996, 795). In particular, facial expressions that are based on elemental features, such as eyebrows, eyes, gaze, mouth, furrows, and wrinkles, or the head position, are conventionally exploited as signs of emotion, thought, attitude, and stance. Similarly, speech in comics, while it may seek to be verisimilar and can provide the linguist with useful examples of spoken language, can take on wilfully distorted forms, such as simplification or exaggeration, that are different from uses of spoken language in real-life speech situations. ${ }^{3}$ As dialogue in comics also necessarily has a written form and often an ostentatiously graphic and handwritten quality, the study of speech in comics needs to be sensitive to graphic features and the visual effects of written language.

Rodolphe Töpffer, who many see as the inventor of modern comics, claimed in his essay "Essai de physiognomonie" (1845) that a graphic trace has unique expressive potential, especially in relation to the drawing of a human face. For Töpffer, all faces in drawings, however, naively or poorly completed, even in the form of simple scribbling, possess a fixed expression. He further surmised that the viewer can recognize such expressions without education, knowledge of art, or any experience in drawing a face. ${ }^{4}$ Similarly, one of the basic tenets in today's psychological research in face recognition is that people identify faces from very little information. In such identification, as in recognizing an emotional expression, the eyes and eyebrows are among the most salient regions to pay attention to, followed by the mouth and the nose (Sadrô et al. 2003; Sinha et al. 2005). Töpffer saw, similarly, that in order for the drawing of a face to be effective, one needs to focus only on a limited number of key aspects, such as the eyes, eyebrows, nose, nostril(s), chin, forehead, wrinkles or folds of skin, and the shape of the head. ${ }^{5}$ Töpffer also thought that the relation between these facial features and the person's posture - the form of his or her upper body, gestures, and attitudesmattered, even though he did not see them as important as the internal features of a face (the eyes, nose, and mouth).

As recent psychological and sociological conversation analysis has shown, facial expressions can enhance or disambiguate the speaker's 
and the recipient's stances towards what is being said in real-life speech situations (Ruusuvuori and Peräkylä 2009). In comics, likewise, a basic element of speech representation is the relation between verbal utterances, facial expressions, and other features of body language such as eye contact, typically accompanied by the sense of perspective and field of vision that are inscribed in the image. For instance, a way of speaking and listening can be revealed by an exchange of looks in subsequent gaze images, images portraying someone looking at something or someone, or reaction images, that is, images showing someone's reaction to something that is said. A recipient's look can, for instance, indicate pensiveness, concentration, or confusion, affiliation with the topic or the speaker, the sharing of an understanding, or the rejection of an idea, or it can reveal what is important and salient in the conversation situation as a whole. ${ }^{6}$

Notice, for instance, the significance of facial expression, gaze, body language, and hand gestures in this scene from Abel Lanzac and Christophe Blain's Quai D'Orsay. Chroniques diplomatiques II (Weapons of Mass Diplomacy 2012), which depicts a meeting between the French Minister of Foreign Affairs, Alexandre Taillard de Vorms (inspired by Dominique de Villepin), his speech writer Arthur Vlaminck, and a representative of the logistics department, Gilles Mande (Figure 9.1). In this scene, the furious minister protests to Mande about not being able to have a bigger aeroplane (Airbus) for himself and his advisors on a diplomatic visit to Russia. The intensity of the minister's gaze and his facial expression, emphasised in the close-up image framed to show only his piercing eyes and part of his gigantic nose, convey the persistence of his stance, as well as his manipulative attitude towards the others. The minister pours forth a tirade of complaints, evidently fuelled by a sense of self-importance, about the tightness of space in the smaller Falcon aircraft that has been offered to him and his staff. All this is accompanied by expressive and manipulative hand gestures.

Besides facial expressions and gaze, hands, hand gestures, and arm positions can also have a significant function in speech situations in comics, communicating meaning themselves or specifying the words' meaning. Two likely reasons for the significance of hands in comics are that we can gesture meaningfully and simulate shapes and things much more accurately with our hands than with other body parts, and that they can relatively easily be drawn to demonstrate this. ${ }^{7}$ Hand gestures may be used as forms of illustration, specifying a type of action, a spatial relation, or a physical shape of something, or as a form of emphasis, while a hand can also point to an object, place, or the interlocutor. Waving, pointing, and beckoning can have a conversational function, for instance, as an expression of the participant's emotion, attitude, and personality, and also as a conversational signal. The salience of hand gestures in the image, or facial expressions, for that matter, can be 

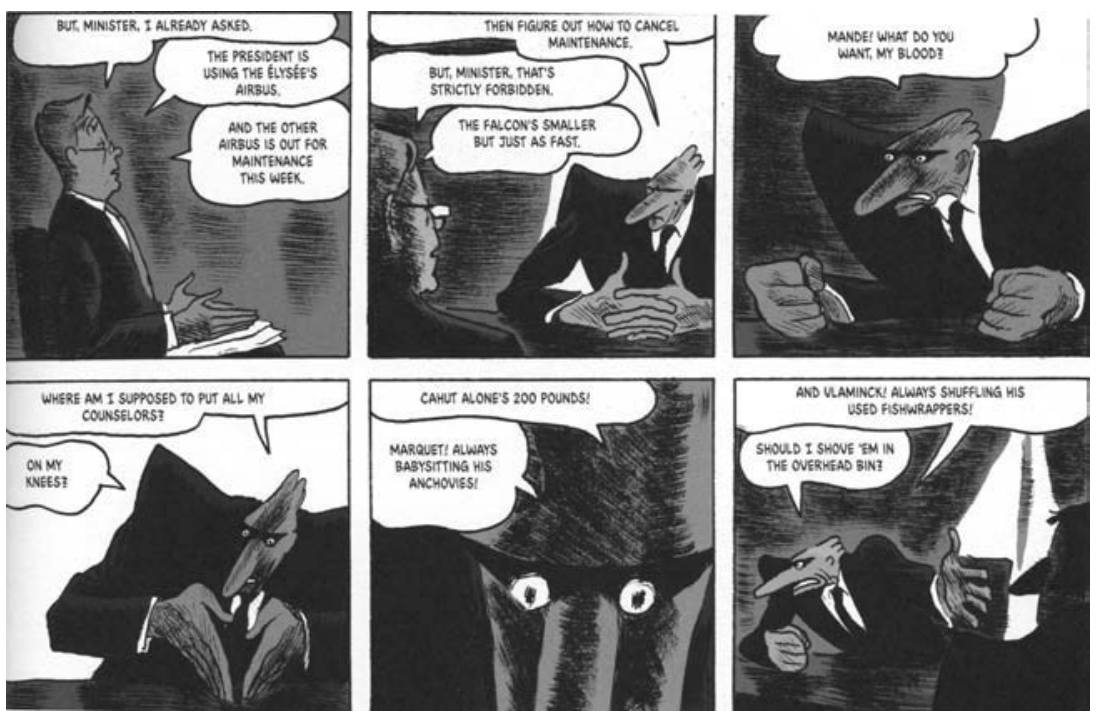

Figure 9.1 Abel Lanzac and Christophe Blain. Weapons of Mass Diplomacy (C) DARGAUD 2010, by Blain (Christophe), Abel Lanzac.

further emphasised by means of layout, perspective, foregrounding, or visual means of emphasis.

The meaning of the participants' positions in an interaction, and what psychology calls (inter)personal space behaviour or proxemics-how people use the personal space around them as they interact with otherscan be effectively portrayed in comics by showing how participants in a scene of talk take their space or relate to each other and the surrounding environment. Focus on a particular person in a close-up image or framing the image close to a participant or his or her field of vision may also suggest a (narratorial) sense of proximity to that participant. ${ }^{8}$ This is also common in film narratives. A more medium-specific aspect of significant body language in comics is the non-realistic manipulation and distortion of body shapes through caricature, that is, the relative malleability of the drawn body. We can observe this, for instance, in the above example from Weapons of Mass Diplomacy where Alexandre Taillard de Vorms's shoulders and nose change their proportional size from panel to panel. The speaker's body is thus modified to reflect his speech, attitude, and personality; the body has an expressive function in itself.

Conversational scenes in comics, as in film narration, have an advantage over dialogue scenes in literature in that they may show various non-verbal communication cues, which co-occur with verbal communication and can combine the effect of such cues. All visually observable aspects of non-verbal communication that may be integrated in a 
face-to-face dialogue in real life can also be portrayed in comics: facial expression, posture, gesture, eye contact, touch, adornment, physiological responses, position and spatial relations, personal space, locomotion, and setting. ${ }^{9}$ While comics, at least in the traditional forms of printed strips or books, cannot usually represent sounds, they have developed various ways of suggesting auditory signals and vocal behaviour, such as onomatopoeia, sound effects, and symbols. All these cues are potentially relevant in conversational scenes in comics, where they co-occur with the verbal utterance. As they interact with each other and the utterance, these devices help the reader to create a sense of a continuing speech event, or what is meant by what is said; better perceive the participant's mental state, attitude, and intention; and grasp the nature of the relation between the speakers. Yet, the ways in which cartoonists may take advantage of the rich possibilities of non-verbal communication in the medium vary greatly. For instance, while facial expressions are generally important, from children's comic strips to adult-oriented graphic novels, or from superhero comics to nonfiction reportage, some cartoonists also simplify facial expression cues or minimise their use. ${ }^{10}$ Thus, the varying aspects of non-verbal communication, and in some cases even facial expressions, can be conceived of as optional tools of visual showing and narration in conversational scenes.

\section{Symbols of the Speaker's Mental State and Engagement}

In much comics storytelling, the use of visual symbols and verbal-visual signs that emanate from the characters may also contribute significantly to speech representation and dialogue scenes. In the passage of Weapons of Mass Diplomacy above, Mande's heavy sweating, shown with drops of sweat, his changing facial skin colour, and later also his gradually shrinking head and body clearly point out his submission to the minister's authority. The visual symbols around his head, which the cartoonist Mort Walker has called 'emanata' and John M. Kennedy identified as 'pictorial runes' $(1982,600),{ }^{11}$ portray emotions (agony), mental states, and an internal condition (submission). These and similar graphic devices, such as drops of sweat or more symbolic signs such as wiggly lines, starbursts, circles, halos, and clouds, often have little or no relation to the outer signs of emotion and attitude in real-life speech situations. As conventions that are used in modern narrative drawings from cartoons to comics, emanata are metonymically motivated signs that result from a character's emotion and thought or some immediate sensory stimuli and effect. Typically, they specify the force of the speech act, a speaker's enthusiasm or uncertainty, the recipient's understanding or lack of understanding of what is said, acceptance and disappointment, or, as here, gradual submission to the speaker. Emanata and altered body shapes can also portray types of perception and reactions, including the sense 


\section{Speech and Thought in Narrative Comics}

of cold and warmth, smell, newness, light, and brightness or perceptions of speed, reflection, sudden or fast movement (speed lines), the direction of movement, or surprise and suspense. Not all comics employ them, but when they are used, they can contribute significantly to our understanding of the other elements in a scene of talk such as facial expression, gestures, and gaze.

Beyond the emanata, or pictorial runes, conversational scenes in comics can also comprise various other signs, including stand-alone punctuation marks, ${ }^{12}$ pictograms, ${ }^{13}$ sound effects, imitatives, and onomatopoeia ${ }^{14}$ that have similar or related functions. Placed in the space in the image around the characters, or possibly continuing from panel to panel, these signs can equally specify the characters' emotions, thoughts, and attitudes or a way of acting, behaving, and speaking; clarify what is said; or express movement, sounds, and other sensory stimuli that are relevant in the scene.

Comics imitatives, which are widely used for humorous purposes, approximate non-linguistic sounds and action or contact between the characters, as well as attitude, emotion, sensations, and movement by adapting them to the phonemic system of the language. Onomatopoeia and sound words (or descriptive sound effects), which can be regarded as a specific case of imitatives, represent sound and voice in verbal form and, at the same time, often aim for a visual effect, which in itself can mime some quality of the sound or reflect its source, such as an event causing the sound. Onomatopoeia may also indicate variation in sound effects such as volume, pitch, timbre, and duration. Typically, onomatopoeia fit the phonology of the language in which they are used ('boom!', 'wham!', and 'whoosh!' in English or 'baoum!', 'pff!', and 'vlan!' in French). In comics storytelling, however, it is also common that an onomatopoeic adaptation of a sound does not necessarily have to constitute a word or even be pronounceable. Onomatopoeic expressions in comics are not usually reducible to the sound that they imitate-one reason being that they are given a visual, graphic form that contributes to their meaning and effect. The use of stand-alone descriptive words (or descriptive imitatives) for sensations and emotions is also common ('snort', 'gasp', 'tickle', 'sigh', etc.).

Stylistic elements of writing, such as lettering, typography, and fonts, as well as what has been called para- or quasi-balloonic phenomena, ${ }^{15}$ can be incorporated in a dialogue scene for similar purposes. The graphic style in which speech is written is often meaningful in such scenes in two senses. First, the graphic style of writing can create an effect of continuity between the world of the story, or the speech situation, and the written speech. For instance, written speech can be placed and shaped in the image field so that it reflects the visual contents of the image. ${ }^{16}$ The graphic line that depicts the speaking figures can also give the impression of continuity in the writing (or vice versa). Second, the style of writing 
can in itself express certain aspects of the utterance, such as emphasise the meaning of a word, a phrase, or an utterance through bold lettering, convey humour, add a metaphorical or ironic layer through a stylistic change, imply a way of speaking or type of voice (whispering, singing, a broadcast voice, and so on), the intensity of speaking (by changing the letter size, for instance), and the speaker's attitude or emotional state. It can also portray differences between the speakers' register, style, or voice. Not all comics use the rich graphic potential of writing in this regard, but the style of writing and the choice of typography are important features of conversational scenes in many comics. Think, for instance, of Walt Kelly's Pogo, or Neil Gaiman's The Sandman, where typographical choices may reflect the characters' personality or attitude, or René Goscinny and Albert Uderzo's Asterix, where changes in lettering can indicate important vocal and linguistic differences in the characters' speech (accent, dialect, stylistic register, language). By these means, written dialogue in comics can overcome some of the limitations that affect the representation of spoken language in conventional literary fiction. ${ }^{17}$

All in all, the various visual and verbal-visual signs that have become conventionalised in comics can be metaphorically motivated as indexes of a speaker's emotions, thoughts, attitudes, and perceptions. All these features may also contribute to the meaning of what is said, and potentially influence the reader's attribution of mental states to characters. Frequently, such signs work together to identify the speaker's attitude, complementing the meanings of facial expression and body language, and thus specify or enhance the speaker's relation to the propositional content of the utterance and the other participants in the scene. ${ }^{18}$

Let us take as an example the main components of a scene of talk in Finnish cartoonist Aapo Rapi's (auto)biographical narrative Meti (2008). This story is based on the cartoonist's interviews with his 80-year-old grandmother Meeri Rapi, known as Meti, but it also has a strong autobiographical dimension: the cartoonist pictures himself in the story, meeting and conversing with his grandmother, taking notes during the conversation, and relates some other events in his life at the time of the interviews and the storytelling. The narrative perspective of Meti is often ambiguous in that there are clues in the story that let the reader think that it is told and illustrated in the way that Aapo imagines the events have happened-Meti's story would thus be within the frame of Aapo's imagination-but there are also passages in the narrative where Aapo's story and Meti's memories appear to be in competition. At times, the frame narrative and Meti's narrative also coalesce, resulting in a kind of intersection of stories.

Here, in this scene of five panels, the speaker's and the recipient's facial expressions, posture, gaze, exchange of looks, perspective, and emanata play a vital role together (Figure 9.2). We first see the cartoonist meeting with his grandmother. When Meti attempts to formally introduce 

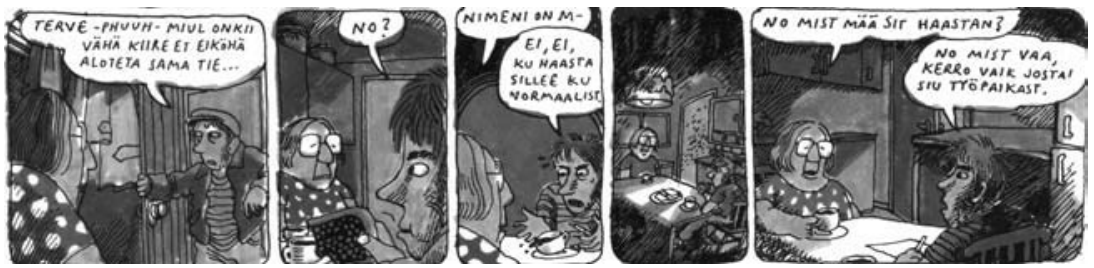

Figure 9.2 Aapo Rapi. Meti (2008) (C) Aapo Rapi.

herself with 'My name is M-', the cartoonist, visibly frustrated by this introduction-indicated by drops of sweat springing from his face, accompanied by a few drops of coffee spilled from his cup-interrupts her and insists that she should speak as she 'normally' does, that is, not in formal discourse. Consider also the importance of gazes and perspective in this scene. Both speakers are present in all images, but seen from different angles and distances. The alternating perspective of the images allows us to see the scene from behind both characters' shoulders and thus share their viewpoints to some extent. Notice also that the cartoonist's face is much more expressive of emotion and mental state-changing from signs of haste and frustration to calm-than that of the stonyfaced main character. Moreover, Meti's large non-reflective glasses are in stark contrast with the youthful expressiveness of her face in the narrated memories that follow this scene.

\section{The Bond between the Speaker and the Utterance}

Speech and thought balloons were successfully incorporated into American newspaper comic strips in the 1890s. In earlier European comics and cartoons, the same device had already been widely used, including British satirical broadsheet prints (1770-1820), but in Töpffer's and in many other mid-nineteenth-century European cartoonists' works, speech was usually represented in captions that were placed underneath the images. Only by the 1940s and the early 1950 s did the representation of speech in speech balloons become a dominant convention in the medium in most Western countries. ${ }^{19}$ Since then, other options for representing direct speech, such as speech quoted or summarised in captions, have remained in relatively limited use. Many contemporary cartoonists, however, represent utterances without resorting to speech balloons. For instance, in Brecht Evens's graphic novels and in much of Claire Bretécher's work, the utterances are simply placed physically close to the speaker in the space of the image, possibly but not necessarily accompanied by a tail that connects the utterance to the vocalizing source. 
Regardless of whether comics use the speech balloon format or not, the general principle that an utterance is tied to a source that is shown in the image or to a source that is situated close to what is shown appears to be a default expectation in comics. The tail emanating from the balloon, or in some cases from the text without a frame, makes this association even more evident as it directly points to the source of the utterance. If the speaker is not shown in the image field, the default expectation is that the balloon and the tail indicate that someone is just outside the visible space or is not yet or no longer in the field of vision, or that the source of the utterance is too small or hidden to be seen (see also Forceville et al. 2010, 69).

Thus, the speech balloon and its tail, which can take a variety of different visual forms, express the contents of the utterance and, at the same time, are visual symbols of a speech act. In the latter function, we need to underscore their metaphorical function, which has something in common with metonymy: the balloon and its tail stand for a speaking voice (or a sound), the place, time, and duration of speaking, and the act of speaking itself. The relationship between the balloon and the speech act can thus be conceptualised as a structure of contiguity where, with the written utterance representing spoken language, the visual form of the speech balloon stands in a metaphorical relation to the source of the voice and, possibly also, to particular aspects of that voice or sound (intonation, for instance). In contrast, thought balloons represent the speaker's thoughts and inner state. The distinction between speech and thought balloons is not always unambiguous in comics, or their difference may be irrelevant-does it always matter, for instance, whether a person speaks or thinks aloud to himself?-but in general they are distinguished by various visual markers such as the shape of the balloon and the tail or the background colour.

Being a visual metaphor (or metonymy) for a speech act, the balloon and its tail also perform the function of speech tags. In fact, they can realise the speech tag function much more efficiently and economically than any verbs of saying that traditionally introduce an utterance in literary narratives. The function of the tail, specifically, is to identify the speaker in the image. ${ }^{20}$ The balloon and its tail not only point out the turn-taking, the source of the utterance, and the place of the speaker, but often also tell us how someone is speaking-the intonation, intensity, and volume of speech may be reflected in the shape, size, place, or colour of the balloon and its tail-or reveal the speaker's attitude towards what is being said (linguistic modality). Balloon frame styles, background colour, and tail shapes regularly depict emotional states and sensory experiences (uncertainty, (dis)approval, 'warm', 'icy'), a type of voice (electronically relayed, distant, shrill, high, low, harsh, broken, and so on), or volume (loud, quiet, shout, whisper). Lettering, typography, and visual signs inside the balloon can have similar functions or can amplify 
them. If in the Asterix albums typography can be a sign of a different language and dialect; in Brecht Evens' graphic narratives, the colour of the text identifies the speaker (The Wrong Place, 2009; The Making Of, 2011; Panthère, 2014).

The expressive uses of the speech balloon are well known to comics readers and scholars, but perhaps less to academics who study the dialogue form across media. Charles Forceville has shown how different visual variables of comics balloons-contour form, colour, fonts, non-verbal contents, and tail use-contribute narratively salient information, for instance, with regard to the manner and topic of speaking or the identity of the speaker $(2013,258,268)$. In other words, the visual variables of the balloon, especially in more nonstandard cases, make salient something in what is said, how something is said, or who the speaker is. This, again, requires that we evaluate the relation of the balloonic narrative information to the speaker and the speech situation as a whole. The place of the balloon in the scene or the breakdown may also be significant. Thierry Groensteen, who has made a theoretically grounded description of speech balloon functions in comics, has suggested that the place of the balloon is always relative to three different elements in the space of the page: the character who is speaking (the speaker), the frame of the panel, and the neighbouring balloons (situated in the same panel or a contiguous one) $(2007,75)$. Groensteen emphasises, in particular, the interdependence between the characters and the balloons $(2007,75,83)$, claiming that their relationship is so strong that they form a sort of functional binomial, a bipolar structure that is a necessary organising device in comics. Moreover, Groensteen presumes that the characters in the panels are the most salient piece of information and, subsequently, echoing Töpffer, that the character's face and physiognomic expression are the principal focal points of the reader's attention $(2007,75-76)$. In reading comics, then, the reader would supposedly first view the character's face and expression, and then adjust this information, reciprocally, with what is said, that is, the character's represented speech. ${ }^{21}$

The claim about the bipolar structure between an utterance and an utterer seems highly relevant with regard to most comics. The psychological study of face recognition has also proven that the human (biological) visual system starts with a rudimentary preference for face-like patterns, and that our visual system has unique cognitive and neural mechanisms for face processing (see Sinha et al. 2005). Yet, it seems worth asking whether the functional binomial between the speaker and the utterance is always dominant in guiding the cartoonist's or the reader's understanding of conversational scenes, or the order of their reading. For one thing, we still cannot say much that is not controversial about the reader's order of attention in reading comics. Do we always start reading comics by viewing the characters' faces? ${ }^{22}$ Comics can vary greatly with 
regard to the relative amount of words they use, as well as for what purpose they use them (what kind of information is given verbally), let alone that the image-word ratio typically alternates within any given story. A dialogue scene can portray the participants' positions, gestures, and relations in great detail, but in a 'talking heads' story or a verbal gag strip, words can also be the primary focus of the reader's attention, whereas sometimes faces can tell next to nothing.

In addition, comics can successfully sever the relation between the speaker, words, and space of the speech situation by various means. This may be done, for instance, by excluding the speaker from the space of the image or the narrative level, by multiplying the number of speakers or utterances, and by making the connection between an utterance and a speaker ambivalent in the space of the image. ${ }^{23}$ The relation between the utterance and the vocalising source may remain deliberately ambivalent, for instance, in panels where there is only speech and the characters are not seen, or not clearly seen, such as in panoramic images where the speaking figures may be shown far in the distance or are not visible at all, or in images where the vocalising agent is visually blocked. François Ayroles's strip "Feinte Trinité", which includes only speech balloons and no figures, pertaining to a conversation between a son, a father, a mother, and God, or the online comic strip Bande pas dessinée, challenges the basic bipolar structure further by never letting us see who is speaking.

Another challenge to the bipolar structure arises from the speaker's ambivalent positioning between the picture space and outside it. In some rare cases, the speaker can also remain systematically absent from the images. Consider, for instance, the continuous commentator track in Altan's Ada (1979) where a speaker, who is never seen, is emotionally involved in the narrative as its commentator and viewer. Much more common is that a voice may, once connected with a particular speaker, become disconnected from that speaker on the visual level of narration. This may occur, for instance, when utterances are superimposed on what is seen in the images, thus suggesting that what is seen is the character's subjective vision. Towards the end of the frame narrative of World's End, the Chaucerian story arc in The Sandman series, the voices of a group of characters at an inn called World's End are superimposed in speech balloons on a double spread with images of an enlarging window pane through which they apparently look at a spectral funeral procession in the sky. The reader, thus, is invited to share their field of vision through the dialogue.

Still other challenges to the rule of the bipolar structure of speech in comics include the multiplication of speakers for one utterance and the use of one speaker as a representative of a group of speakers. For instance, Martin Cendreda's one-page story, "I want you to like me", experiments with this principle by letting a conversation continue from panel 


\section{Speech and Thought in Narrative Comics}

to panel while the speakers and their spaces keep changing (Chapter 3). This creates the effect of a communal mind that apparently thinks the same thought, and says the same thing, irrespective of the individual sources of utterance seen in the images (speakers, billboard, dogs). Similarly, ideas apparently voiced by one person can be attributed to a group of people. ${ }^{24}$ A character's voice may also occur in many parts of one panel. This can emphasise, for instance, the speaker's quick movement, the effects of an echo, or the complexities of space, as happens in Asterix and the Banquet when the Gaul Jellibabix, who is not seen in the panel, says 'Here!' in six different corners of the maze-like alleyways of Lugdunum (modern-day Lyon) seemingly at the same time.

All these cases experiment with the basic expectations of speech representation in comics: an utterance is visually tied to a particular speaker, and both the utterance and the speaker belong to the space that is seen in the panel. Yet as the exceptions above show, the bipolar structure between the speaker and the utterance can always be modified, challenged, and even discarded. The exceptions make the rule more visible, but the flexibility of the structure also points out that, to better understand speech representation and dialogue in comics, it is crucial to think beyond the speaker-utterance relation to a number of other seminal elements of dialogue in the medium.

Still another important feature of conversational scenes in comics is the interaction between the utterance, the contents of the image and narrative captions. Narrative captions, which are typically distinguished from speech balloons by their frames, background colour, or typography, can also complement, evaluate, or interpret the speech acts presented in the images. In Daniel Clowes's first-person narrative Mister Wonderful (2011), the contrasted and sometimes competing thought captions and speech balloons of the story make clearly visible the expected interrelations between the captions and the balloons. Here, the narrator's thoughts, placed in square-shaped captions with a yellow background, are frequently superimposed on speech balloons that contain the narrator's own speech or other people's utterances, thus indicating, among other things, the narrator's lack of attention to what is being said. On a few occasions, the speech balloons are also superimposed on the captions, thus suggesting that what is said interrupts the flow and momentum of the narrator's thoughts. Thus, also, the connection between the speaker and the utterance in the balloon is momentarily broken.

\section{The Temporal and Rhythmic Functions of Speech Balloons}

Having investigated some basic formal elements of speech representation and scenes of talk in comics, we should be able to focus more specifically on how some of these elements realise narrative functions in comics. 
Character-to-character dialogue, or combined action and dialogue scenes, are central forms of narrative organisation and development in comics, as in literary fiction and film. ${ }^{25}$ Dialogue scenes move the story forward, for instance, by giving important information about the characters, their relationships, the milieu, and the evolving events; they can also build suspense and reorientate the narrative. In comics, dialogue also regularly accompanies action. In Asterix, much of the talking between Asterix and Obelix, which is a constant feature of the series, takes place when the two characters are on the move or doing something. Action and dialogue are constantly bound together: while moving or acting out a scene, the characters discuss their intentions, thoughts, and emotions or voice comments about an event or someone they have met.

What Sarah Kozloff has outlined as the main narrative functions of dialogue in film largely apply to comics. Dialogue in films, as Kozloff points out, can contribute to many if not all key elements of a narrative: world construction and identification, characterisation, communication of narrative causality (such as the relation between events or the significance of an event), enactment of a narrative event (the disclosure of important information such as the speaker's emotional state), adherence to realism (plausibility), and control of the viewer's evaluation and emotions (the sense of narrative rhythm, the effects of surprise and suspense) (2000, 33-51). Inevitably, a given instance of dialogue can fulfil several of these functions simultaneously.

What is different in comics in this respect may to some extent be self-evident. Comics lack the sound element, the means and possibilities of the moving image, and the actor's work and personality is not an issue. With regard to narrative pacing and rhythm in comics, speech balloons play a vital role. Their arrangement in the panel, a sequence, or on the page, modifies both the sense of the time of the narrative and the order and time of reading. On the one hand, the utterances punctuate the story and the dialogue scene and, thus, create a sense of the duration of the event. Sometimes, the speech balloons can in themselves express duration through elongated forms of tails that surpass the frame borders. On the other hand, the speech balloons are part and parcel of the spatial organisation of the comic's page. While the speech balloon constitutes a space where the utterance can be read, the placement and interrelation of the speech balloons in the space of the page also point out to the reader an order of looking and reading, functioning as one means of connectivity between the panels. From the reader's perspective, thus, the utterances in a given narrative comic mark stages in the story that need to be attended to. ${ }^{26}$ Speech balloons placed on the picture frames, for instance, or close to each other in neighbouring panels, can strengthen the link between the pictures and thus affirm the order of reading. Sometimes also, the space of the utterance can approximate the function of a picture frame or the space between the panels. The placement of 


\section{Speech and Thought in Narrative Comics}

speech balloons in a scene of talk may also emphasise, together with other features of the scene, particular aspects of the utterance and the speech situation.

The opening scene of Book One in Garth Ennis and Steve Dillon's Preacher (1996) features a conversational scene between three characters, Jesse Custer, Tulip O'Hare, and Cassidy, who are conversing at a table in a diner in Texas. In this example, I would like to emphasise the significance of three factors in the depiction of the scene: the place of the utterance, the effect of the moving perspective, and the means of layout. The first time we see the protagonist Reverend Jesse Custer's face and his clerical collar, his utterance-'cause lemme tell you: it sure as hell ain't the church'-is placed over the frame border. Both the placement of the utterance, the particularity of which is emphasised by the fact that speech balloons very rarely cross the panel frames in this series, and the contrast between what Custer says and who he is stress the importance of the utterance (Figure 9.3). Further noteworthy elements in this panel are the angle of vision, which is placed squarely amidst the interlocutors and very close to Cassidy's position in the scene, and the fact that two sides of the panel bleed off the corner of the page. The latter feature may compel the reader to turn the page to learn more about the contrast between the speaker and what he has said. In the following pages that depict the conversation, the perspective remains close to the characters, stressing the meaning of gazes and the exchange of looks. Moreover, and typically of dialogue scenes in many contemporary graphic novels, the perspective keeps steadily shifting around the conversing characters, moving to one more or less subjective angle of vision in each panel. Finally, page layout also contributes to this scene through the partial superimposition of some of the panels, such as a close-up image of Tulip O'Hare, on the surrounding panels, thus further aligning the interlocutors to each other and emphasising the importance of a particular gaze, expression, and utterance.

Concerning the sense of rhythm in such scenes, one default expectation is the correspondence between the utterance or an exchange of dialogue and the speaker's (or listener's) posture shown in the image. We could call this the realistic formula of time in a scene of talk. In other words, perhaps the most basic rhythm of speech representation in comics is one utterance per speaker, or one utterance and response per panel. Will Eisner, for instance, has stressed the importance of preserving such a bond between dialogue and action on the grounds of realism, claiming that a protracted exchange of dialogue cannot be realistically supported by unmoving static images. Furthermore, for Eisner, a verisimilar exchange of dialogue is one in which the utterances terminate the endurance of the image, that is, the dialogue corresponds with the speaker's (or speakers') posture in the image $(1996,60)$. 


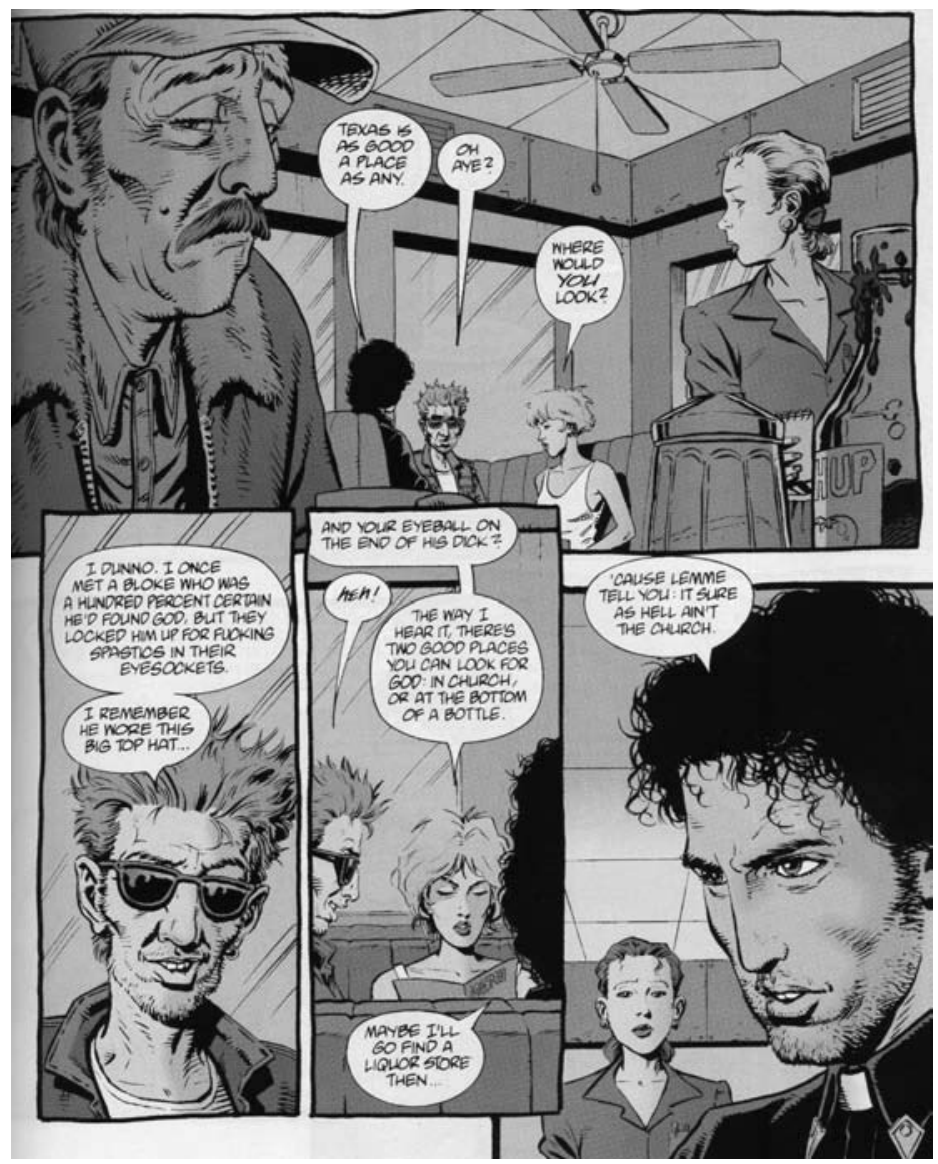

Figure 9.3 Garth Ennis and Steve Dillon. PREACHER. Book One (1995) (C) Garth Ennis and Steve Dillon. All characters, the distinctive likenesses thereof, and all related elements are trademarks of Garth Ennis and Steve Dillon.

However, Eisner's presumption, while it illustrates a basic convention for representing duration in conversational scenes in much comics storytelling, can be contested as an all-encompassing general rule of realistic speech. Clearly, instead of undermining the sense of veracity in a conversational exchange, a long string or multitude of balloons in one panel can also enhance realism in narration. On the first page of Preacher, Cassidy's and Tulip O'Hare's utterances have two parts-their difference is marked, respectively, by the conventions of one balloon opening onto another and by a connecting tail between the balloons. This is a common way to indicate a short pause in speech. Elsewhere, 


\section{Speech and Thought in Narrative Comics}

the placement of many speech balloons in one panel can create the effect of a speeded-up and intensified exchange of words. Strings of balloons or a mass of balloons in one panel may, for instance, suggest the effect of an improvised discourse, conversational intensity (as in the streets of Lutetia in Les lauriers de César), interruption and talking over others, the volume of speech, a cacophony of voices, and so on. Many superimposed balloons can also indicate a disconnection between speech and thought, as happens in Mister Wonderful, where the narrative captions that are placed on the speech balloons and sometimes even on the speakers' faces emphasise the effect of an inner voice overriding speech. Moreover, a protracted exchange of dialogue in one panel may suggest a notable speeding or slowing of time in a scene of talk, instead of undermining conversational veracity.

Furthermore, it is important to note that the relation between speech and posture does not alone create the sense of rhythm in dialogue scenes. The panel-to-panel transitions and other spatial relations on the page, including the sense of time in a single panel, also affect our understanding of the time and duration of a scene of talk. In our previous example of Meti, the sequence suggests a slowing of time during the dialogue scene: the cartoonist's hurry to start the interview-he is visibly out of breath when he enters the room in the first panel-is contrasted with Meti's relaxed attitude. Meti's calmness has become evident to the reader already in the previous wordless pages of the story, which portray her leisurely picking berries, preparing a pie, and baking it in the kitchen. The fourth and the only wordless panel in this sequence, in which the perspective is more distant and impersonal, powerfully suggests the passing and slowing of time. In these five panels, the cartoonist figure thus apparently adjusts to Meti's sense of time by eating lingonberry pie and drinking coffee. Only then can the actual storytelling start.

Conversational scenes, when perceived as distinct scenes, may alter the temporal rhythm in relation to the surrounding narrative action. This dimension of dialogue scenes in comics corresponds with what Kozloff refers to as the control of viewer evaluation and emotional response through dialogue. In comics, as in film, such scenes can distract, create suspense and surprise, or control emotional response by elongating a moment and stretching out a suspenseful climax or pause. The conversation at the beginning of Preacher, which turns out to be a frame narrative for much of the ensuing story in Book One of the series, introduces us to the main characters and opens up several questions about their situation that will be dealt with in the subsequent instalments of the story. Scenes of talk can also slow down the tempo in the narrative, as in the example from Meti above, to the extent that they give us an impression of simultaneity between the time of the events and the time of their telling and showing. In comics that include extensive dialogue during the action, such as Asterix or other European adventure 


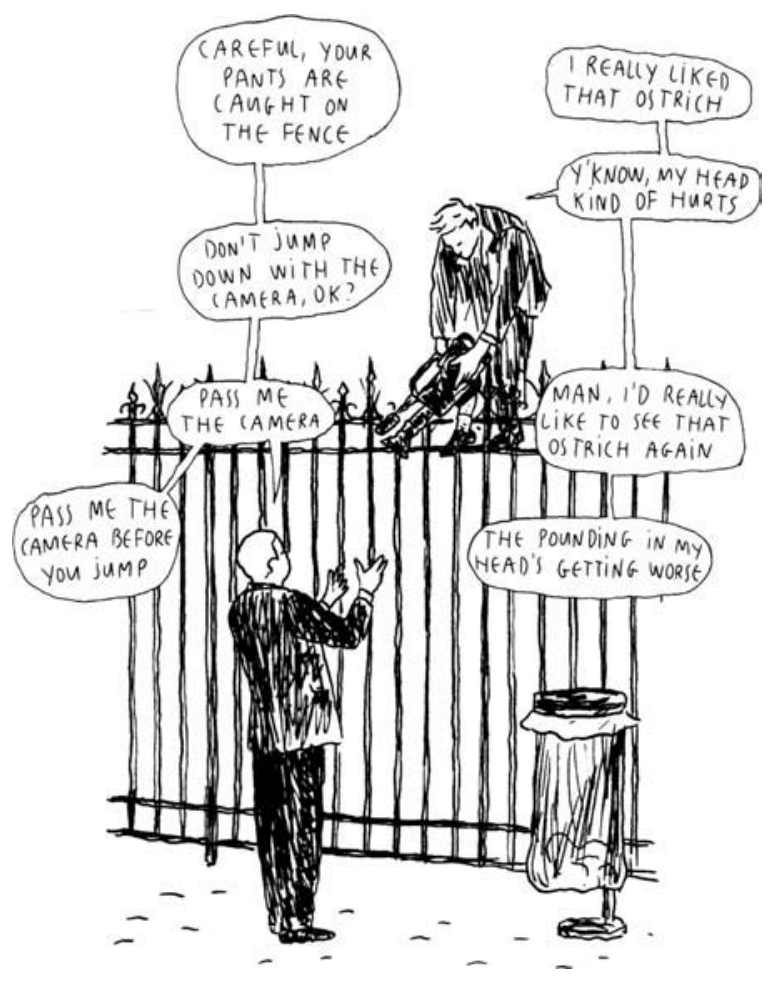

Figure 9.4 Jérôme Mulot and Florent Ruppert. Barrel of Monkeys () 2008, Ruppert, Mulot and L'Association, Rebus Books for the English translation.

series, such as Spirou and Fantasio, such temporal changes may not be apparent, however, since the action and dialogue establish such a steady rhythm throughout the narrative.

Jérôme Mulot and Florent Ruppert's comic books, including Safari monseigneur (2005), Panier de singe (Barrel of Monkeys, 2006), and Le Tricheur (2008), make visible a number of underlying principles in speech representation in comics. For instance, they extend the traditional realistic duration of speech in a panel: Ruppert and Mulot sometimes place up to twenty balloons per panel for one speaker and thus obfuscate the expectation of synchrony between the speaker's posture in the image and the utterance (Figure 9.4). Furthermore, their work investigates the rules of readable information, that is, that speech balloons should contain informative utterances that are attributed to some agent in the story. Generally speaking, certain constraints guarantee the readability of speech balloons in comics. This means that one is to avoid 


\section{Speech and Thought in Narrative Comics}

(a) superimposed speech balloons that block the reading of other balloons, unless the superimposed balloons serve a clear narrative function such as indicating the simultaneity of many voices; (b) balloons placed in a semantically important part of the image (such as the speaker's face); (c) balloons that are 'cut' by the image frame so that they become unreadable (this may also happen in Mister Wonderful to point out the narrator's lack of attention or interest); and (d) continuous nonsensical expressions or empty balloons. However, single 'blah-blahs' or empty balloons can be very revelatory of attitude or a lack of response.

Still other experiments with speech and thought balloons in Ruppert and Mulot's comic books involve the breaking of the flat symbolic space of the speech and thought balloon. For instance, letters and signs regularly overlap the balloon contours and extend to the space of the image in their works, thus undermining the expectation that the balloon is an enclosed space in itself, or speech and thought balloons are treated as literal containers that convey the illusion of three-dimensionality. Some of Ruppert and Mulot's speech and thought balloons, or their contents, can be seen, touched, and entered, whereas others may indicate the speaker's movement in space as a kind of visual trace of the movement.

\section{The Narrative Function of Visual and Verbal Contrast in Dialogue Scenes}

Still another medium-specific aspect in conversational scenes in comics is the narrative effect (rather than function) of contrast, or narratively motivated transition, in the balance between visual and verbal narration. For instance, a scene of character-to-character dialogue in comics can always turn into a predominantly visual narrative that fleshes out the topic of the conversation in narrative drawings, or vice versa. This is a typical element in Aapo Rapi's Meti and complicates in this story the question of the identity of the narrative agent responsible for what is shown in the images. Lilli Carré's The Lagoon (2008), in turn, depicts a scene where someone is telling a tale, and the oral story is then transformed into a visual narrative that the reader can see evolving from panel to panel. The shift from verbal to graphic narration thus dramatises the temporal distance between the present of the storytelling and the past of the story events, but it also has the narrative effect of accentuating the storyteller's skill of inviting the listener into her world and experiencing it from within. Such transferences between verbal and visual narration may in some cases be compared to shifts between different diegetic levels in a literary narrative, for instance when an interlocutor in a conversation becomes a narrator of his or her own story. Yet, the multimodal nature of comics allows the invention of forms of complexity in this regard, pertaining to the relation between the time of the events and the time of their telling, or the source and perspective 
of narration, that are not available in the monomodal context of literary narratives.

Cartoonists can set up tensions between verbal and visual narration in conversational scenes for various other effects as well. Another device for contrasting verbal and visual narration is to juxtapose the time and place of an ongoing conversation and the time and place of the events that are the topic of the conversation. For instance, at the beginning of Jean-Claude Mézières and Pierre Christin's Brooklyn Station Terminus Cosmos (1981), where the main characters Valerian and Laureline are engaged in a long telepathic intergalactic conversation, their dialogue provides the story with a narrative frame. This global frame embeds images from the speakers' memories as short flashbacks as well as illustrations of things and events that the speakers have heard. The extended present moment of the dialogue thus creates a kind of intersubjective consciousness frame that incorporates different temporalities and changes of space, which are shown in the narrative drawings. The dialogue may specify that the things seen in the panels have a varying relation to reality-first- or second-hand information, mnemonic images, or things seen in the speakers' present whereabouts-or different meanings for each speaker. The overall effect, however, is not one of simple framing and embedding, but the time and space in which speakers are situated occasionally also appear to coalesce with those of their stories and memories as the speakers share the imagery through the telepathic link.

\section{Conclusion}

The ultimate goal of this chapter has been an attempt to develop a more general understanding of the basic elements, main compositional principles, and narrative functions of speech and dialogue in comics. One crucial area for future research that is indicated by this discussion is the way in which the image content, especially the embodiment of the participants, contributes to the conversational scene and the interpretative effects that the scene generates. Typically, the images in comics show involvement in scenes of talk through shared or contrasted perspectives, an exchange of looks, or through gesture, posture, and other physical signs of reaction to others. A key aspect of dialogue in comics in this respect is the depiction of the participants' face and facial expressions. Visual symbols and verbal-visual signs, such as emanata, which are added to or around the participants' face and head in some comics, can specify an expression, show mental states, and emphasise a reaction to someone or something that is said. Furthermore, comics may manipulate the characters' body shape and size to underline certain aspects of a speaker's experience, attitude, or personality, or their reaction and engagement in the speech situation. Together and in interaction with the verbal content of the dialogue, these elements produce an integrated, but often quite complex, whole. 
Finally, all compositional and spatial elements in comics can have an expressive function that contributes to the reader's understanding of conversational scenes in this medium. Changing picture frames, panel forms, panel and balloon shapes and sizes, page setup, lettering and letter size, non-realistic backgrounds, ${ }^{27}$ and other components of graphic style can convey relevant information, for instance, by emphasising or modifying the meaning of the utterances or pointing out the salient features in the situation. Moreover, the relations between the panels may imply relevant narrative information about the scene; the gaps in what is visually shown in the panel images need to be related to what is said but also to the gaps in the dialogue. The precise meaning of the potentially meaningful formal elements in a scene of talk depends again on the co-occurrence and combination of these elements and on their tension and interaction with what is said and shown in the images.

Comics share various functions of narrative communication through dialogue with other narrative media, but also employ many medium-specific strategies that render impossible any direct comparison with dialogue scenes in literature or film. Speech in comics is not only given in a written form but also (usually) in a drawn form, a kind of graphic writing. In this respect, comics vary greatly in the extent that they can maximize the graphic and typographical effects of written speech. The speech balloons function as a visual metaphor for a speech act, voice, and source. At the same time, the speech balloon, the tail, and para-balloonic utterances contribute to the organisation of the time of the narrative and the order and time of reading. Above I have also investigated the common convention in comics that an utterance is physically tied to its source, the speaker, and that this relation suggests a certain (imaginary) duration of time. By developing Thierry Groensteen's $(1999,2007)$ insights about the elemental association between the speaker and the utterance, I have sought to contextualise this compositional principle in relation to other key elements of conversational scenes in comics.

\section{Notes}

1 See also Saraceni $(2003,66-67)$ on how this may happen in thought balloons and monologue.

2 Oswalt defines an 'imitative' as "a word based on an approximation of some non-linguistic sound but adapted to the phonemic system of the language" (1994, 293).

3 See also Frank Bramlett, who stresses that a linguistic investigation of language in comics needs to consider the balance of realism in the characters' language and the amount of linguistic exaggeration and simplification that is typical of the medium $(2012,183)$. See also Hatfield $(2005,34)$, Groensteen (2007, 129), and Miodrag (2013, 32-36).

4 The art historian Ernst Gombrich famously named this rule Töpffer's law: "For any drawing of a human face, however inept, however childish, 
possesses, by the very fact that it has been drawn, a character and an expression" (Gombrich 1960, 339-340).

5 Bremond points out how the 'teratological' anatomies of certain characters in comics allow us to pose the question of which bodily organs are absolutely indispensable for the realisation of gestural messages $(1968,99)$.

6 One type of gazing that may be equally well-portrayed in dialogue scenes is the characters' joint visual attention to something. For a reference in film studies, see, for instance, Persson (2003, 68-91).

7 See Baetens (2004) on the depiction of hands in Yves Chaland's and Jacques Tardi's works.

8 See, for instance, Persson on visual media and personal space (2003, 109-110).

9 Speakers in real-life speech situations can co-opt almost any physical action conversationally, that is, demonstrate by timing an action with the verbal communication that the non-verbal act has a communicative function (Bavelas and Chovil 2006, 100).

10 E.S. Tan argues that some graphic novels avoid using the schema of facial expressions altogether, "either because it is too explicit, or because the emotions that characters have are too complex to be 'told' through the face" (2001, 45). I would argue that narration "through the face" is a matter of stylistic choice rather than a reflection of the story's simplicity.

11 Kennedy distinguishes actual pictorial runes that are metaphorical, such as the state of anxiety shown by eye spirals, from graphic lines that have some literal intent as they attempt to convey perceptual impressions, such as lines radiating from bright light $(1982,600)$. Forceville has adopted Kennedy's term $(2005,2011)$. In his tongue-in-cheek lexicon, Mort Walker defines emanata as emanating outwards "from things as well as people to show what's going on", such as a character's "internal conditions" (2000).

12 See also Dürrenmatt's $(2013,115-127)$ discussion of how exclamation points, question marks, and ellipses have become autonomous means of description in the medium, especially for expressing characters' emotions, mental states, and/or silence.

13 Forceville, El Rafaie, and Meesters distinguish a pictogram from a pictorial rune on the basis that an isolated pictogram, such as $\$$ or $\boldsymbol{\vee}$, has "some basic meaning of its own when encountered outside of comics", unlike a pictorial rune such as motion lines, droplets, spikes, or spirals (2014, 492-493). They admit, however, that the borderline between the two categories may be fuzzy $(2014,494)$.

14 Suzanne Covey distinguishes between 'descriptive' sound effects, by which she means "words, usually verbs, that don't attempt to reproduce the sounds they depict" and onomatopoeic words that try to approximate sounds at least to some degree (2006).

15 Forceville, Veale, and Feyaerts include in para- and quasi-balloonic phenomena the various non-bordered zones of the picture that display onomatopoeia and sound effects $(2010,65)$. On onomatopoeia in French-language comics, see Fresnault-Deruelle (1977, 185-199).

16 Some examples are discussed, for instance, in Dürrenmatt (2013, 165-167).

17 Compare with Chapman $(1984,18-24)$ on the difficulties of reproducing speech in written dialogue.

18 Forceville emphasises, importantly, the combined effect of non-verbal signs in comics in the representation of emotions such as anger $(2005,84-85)$.

19 See Smolderen $(2002,2009,119-127)$ on why the speech balloon was rarely utilised as a citation of a character's speech before Richard F. Outcault's 


\section{Speech and Thought in Narrative Comics}

"The Yellow Kid". There are important exceptions, however (see the last chapter of this book). Lefèvre discusses the gradual spread of the balloon device in European comics since its final breakthrough in the 1930s (2006).

20 Saraceni argues succinctly that the "function of the tail is equivalent to that of clauses like 'he said' or 'Ann thought' in reported speech or thought" $(2003,9)$.

21 Lawrence Abbott's educated guess about eye movements and the order of reading comics is similar to Groensteen's suggestions, but Abbott puts the main stress on words and verbal narration (1986, 159-162).

22 Will Eisner's caution in this matter seems justified, even if eye-tracking research has made important advances recently: "In comics, no one really knows for certain whether the words are read before or after viewing the picture. We have no real evidence that they are read simultaneously. There is a different cognitive process between reading words and pictures. But in any event, the image and the dialogue give meaning to each other-a vital element in graphic storytelling" $(1996,59)$.

23 See also Forceville (2013), who discusses some effects of tailless balloons and tails that do not point toward an identified or identifiable speaker.

24 Carrier $(2000,42-43)$ associates this effect with a page from Joe Sacco's Palestine, but does not explicate how the effect is created. See also Forceville (2013, 265-266) on a panel in Régis Franc's Nouvelles Histoires: Un dimanche d'été, where a substantial number of tails do not point toward any identifiable speaker, thus creating the effect of a palaver where "it does not matter very much who is saying what".

25 See also Phelan and Rabinowitz (2012, 37-38).

26 In Groensteen's formulation, the positioning of the balloons in the space of the page creates a rhythm in reading as "each text fragment retains some moment of our attention, introducing a brief pause in the movement that sweeps across the page" $(2007,83)$.

27 On how pictorial metaphors in the image background may express a person's emotional state in manga, see Shinohara and Matsunaka (2009, 283-290). 


\section{Part V \\ Narrative Form and Publication Format}


This page intentionally left blank 


\section{Picture Story and Narrative Organisation in Early Nineteenth-Century British Caricature and Comic Strips}

Just as it is the task of the literary historian to evaluate in what way and how far the term "novel," in the nineteenth-century sense, can be applied to an example from the sixteenth or seventeenth century, so it is our task to determine how far the term "narrative," as exemplified in the nineteenth- and twentieth-century strip, really fits a given sequence from an earlier period.

—David Kunzle $(1973,5)$

Comics are a continuing saga, and there lies the rub: there is no point in their history where we can pick up a particular paper and proclaim it Comic Number One. This makes comics intriguing for the historian, infuriating to the collector.

$$
\text { —Denis Gifford }(1976,6)
$$

Voici mon credo, résumé par une simple proposition: pour l'historien du 9e art, la démarche correcte n'est plus d'expliquer l'histoire de la bande dessinée à partir de la forme que nous connaissons, mais d'expliquer la forme que nous connaissons à partir de son histoire.

—Thierry Smolderen (2012b)

To conceive comics as narratives, and therefore as something that is of interest to narratology, is not only a matter of identifying the narrative forms of the medium as we know them today. Various historically specific circumstances of artistic creation, publishing, and reception also condition the conception of comics as narratives. Such factors include the genre, the publication format, the significance of paratexts, ${ }^{1}$ the evolving intermedial relations, as well as what the cartoonists and the readers expect of stories in this medium in the first place. While these issues may be said to exit narratology proper, that is, the description of the means and techniques of narration, they become highly relevant whenever narratology moves beyond that descriptive task, and engages with the history of the medium and the interpretation of individual works.

The given publication format of comics, ${ }^{2}$ from newspaper strips to trade paperbacks and graphic novels, or from yonkoma (the Japanese 


\section{6}

four-panel comic strip) and manga magazines to tankōbon books, is associated with specific audiences and their expectations about what kinds of stories can be told in that format. The publication format is also tied to expectations involving the time of reading, the kind of reading experience (such as emotional response, suspense, and the like), and genre-related expectations concerning plot, characters and milieu. Furthermore, the format affects the cartoonist's choices on various levels. Such choices may concern, for instance, the amount of complexity in characterisation and plot, the extent of visual detail, the layout style, the relationship between the images and the spatial form of the composition, and the ratio between text and image.

Despite the emphasis on the means and techniques of narration, the narratological enterprise can also be grounded in the historical development of the given genre, publication format, or medium. One approach that has gained some popularity recently in this respect is a diachronic inquiry into the history of narrative forms. This means, more precisely, the investigation of the basic narrative techniques, the functions of these techniques, and artistic solutions, that are available in specific forms of production and publication, genre, or body of work at a particular time. In this chapter, I will pursue such a diachronic investigation by examining the main options that were available for organising a picture story in the historical context of early nineteenth-century British satirical and narrative drawings, published in the new format of the caricature magazines. In this investigation, I will privilege the development of certain formal and compositional features, in particular the conception of panel arrangement and panel relations. Thus, the objective is to show, on the one hand, that narratological inquiry can be significant for understanding the historical relation between the publication format and the means of storytelling and, on the other hand, to argue that the said caricature magazines contributed in important ways to the development of narrative comics as we know them today.

\section{Conceptions of Narrative}

As earlier research in the history of modern comics has shown, the art of caricature in the satirical broadsheets published and circulated in the Georgian era in Britain allowed rich experimentation with graphic style, caricature, and the story form. Gradually, over the course of the nineteenth century, coinciding with the remarkable growth of the print industry at this time, the satirical and humorous drawings of the broadsheet print progressed into the newspaper cartoon and the comic strip. ${ }^{3}$

Hitherto, however, the histories of comics have said very little about the development of the narrative form from a narratological point of view. What makes the caricature and narrative drawings of the early nineteenth-century British context particularly pertinent for our discussion 
is that here, before the demands of mass-produced comic strips, ${ }^{4}$ the space of the composition and the narrative form of the picture story were conceived as a dynamic field of experimentation. In part, this experimentation was enabled by the features and allowances of the new popular printed formats. Many basic formal features of modern comics developed in the satirical broadsheet print, caricature magazines, and illustrated magazines of this time, yet unhindered by standardized forms of the panel strip or expectations pertaining to what 'comics' are. While we may recognise many of these works as comic strips in the contemporary sense, no 'cartoon' or 'comic' was still produced under such definitions and expectations.

The further we go back in the nineteenth century, the more contingent and relative the notion of a 'cartoon', let alone the terms 'comic strip' and 'comics', become. The use of the term 'cartoon' in reference to humorous and satirical drawings published in printed press can be dated back to the weekly Punch that on 15 July 1843 featured John Leech's caricature "Cartoon, No. 1-Substance and Shadow" that we have discussed in an earlier chapter. From then on, in association with the satirical topical drawings in Punch, the term transformed into a general notion for caricature of this kind, potentially also in reference to sequential stories. By contrast, the terms 'comic' or 'comics' derived much later and much more gradually from the comic content of the popular newspaper strips, the establishment of publication formats that concentrated on sequential stories (the 'comic strip' and the 'comic book'), and names of popular titles, such as the British illustrated weekly Comic Cuts ${ }^{5}$ that affirmed the close connection between a drawn story and comical content. The contemporary meanings of the 'comic strip' and 'comics' were established only after World War I. ${ }^{6}$

There is no one historical beginning for comics as a medium or a form of art. Yet, we can locate various important, and sometimes discontinuous, developments of graphic expression and picture story in different cultural contexts, artistic traditions, and publication formats that contributed to its emergence. In his studies on the origins of modern comics, Thierry Smolderen has emphasised the importance of two publication formats that functioned as kinds of cartooning laboratories for modern comics: the caricatural broadsheet prints in the Hogarthian lineage, and the mid-nineteenth-century illustrated magazines in Britain and France. The popular British mid-nineteenth-century illustrated periodicals and weeklies, such as The Penny Magazine, Figaro in London, Punch, The Illustrated London News, The Pictorial Times, and The Graphic, accommodated what Smolderen calls 'polygraphic experiments', graphic solutions in visual representation, and hybridising semiotic practices derived from cartoons (in the contemporary sense of single-image drawings). For Smolderen, the graphic solutions that emerged from these periodicals included, for instance, the development of the clear line and the modelled line, the possibility to combine various graphic styles, 
the schematic depiction of an instantaneous moment, the use of speech balloons, and the art of caricature to depict posture and physiognomic expressions and register shock, surprise, and violent emotion (Smolderen 2012a, 2014a, 3, 2014b, 53-54). These solutions, Smolderen claims, together with Rodolphe Töpffer's ironic novels in print that were first published in 1837, suggest the immediate historical formal context for modern comics, in particular the comic strip.

Importantly, Smolderen problematises the use of an axiomatic definition of comics as a sequential art, and questions the type of comics history that concerns itself only with formats that correspond to a contemporary definition of the medium (2012b, 2014b, 60). What makes the transposition of the idea of sequential art to the nineteenth-century context particularly problematic is that illustrators and caricaturists in Europe at this time were engaged in hybrid semiotic practices with drawing and writing that, rather than developing the sequential form, focussed on means of humor and satire (Smolderen 2012a, 2014a, 50-51).

Yet, what remains open in Smolderen's history of comics, and perhaps overshadowed by his emphasis on the development of the polygraphic visual field and Töpffer's ironic treatment of the sequential form, is the question of the narrative form and function of the new graphic solutions. Much more could be said about the development of the story form and the narrative organisation of picture stories in this historical context, both in relation to the sequential form and beyond.

The question of narrative sequentiality in early nineteenth-century caricature and picture story is more complex than a contrast between a Hogarthian series of images, where the linking between the images takes considerable effort and reading/viewing is necessarily slow, and the emerging mass-produced narrative strip design that enables a more fluid way of reading. The opposition that Smolderen establishes along these lines allows him to argue that the emergence of the modern comic strip in the nineteenth-century illustrated journals, newspapers, and comic magazines pushed to the side the Hogarthian complexity of the picture field. Thus, also, the principles of simultaneity and sequentiality are contrasted: the caricaturists and artists of the new illustrated magazines and newspapers were constantly preoccupied, Smolderen claims, with "the contrast between simultaneous presentation (serpentine and intricate) and sequential content (straightforward action and/or rigid social scripts)" (2014a, 84). The emergence of the newspaper comic strip may then be said to be the culmination of the developments that enabled cartoonists to better link images in series through image content, and thus create the illusion of continuous action and motion, inspired by the new recording technologies of photography and film.

However, it needs to be stressed that in the early nineteenth-century context the sequential forms of graphic art were not restricted to simple linear forms of reading and narrative organisation or a contrast 
with simultaneous presentation. Other relevant kinds of sequentiality include back-and-forth, up-to-bottom, and bottom-to-up sequencing, and a variety of types of spatial contiguity and temporal relations between the panels beyond the linear order, including also forms of visual storytelling that are engaged with the total space of the composition. Moreover, while I agree with Smolderen that the purpose in early and mid-nineteenth-century 'comics' in the illustrated magazines is frequently to make comical points or offer humorous digressions by telling the story in a certain way, instead of trying to tell a story "seamlessly", "effortlessly", or "effectively" (2014a, 15, 129), this does not diminish the interest in investigating the conventions and conceptions of the story form at this time. On the contrary, the historical forms of organising narrative drawings in series become particularly interesting at this time since (1) sequentiality has many varieties in these works; (2) sequential order may be combined with other principles of organisation such as juxtaposition and simultaneity; and, moreover, (3) storytelling in the strip design does not always seem so effortless as may be expected from today's perspective. The sequential form is not a purely axiomatic concept in this context, but can be grounded historically in its multifaceted manifestations, for instance, in relation to particular traditions of caricature, picture story, audiences, and the evolving publication formats.

Furthermore, it is essential to note that the sequential form in the early nineteenth-century British context is both an aspect of layout and image content, and that in both of these aspects, the sequential structure is used by the cartoonists to engage the readers in certain ways. In terms of composition, the sequential form suggests, as a type of reading protocol, that it makes narrative sense to follow a series of images in particular order. The strip design, however, does not have to be based on the sequential form, even if these two usually coincide in comics. ${ }^{7}$ There are, for instance, a rich variety of non-narrative uses of the strip design in the satirical broadsheet prints and the later caricature magazines: descriptive image series, series of caricature portraits or stereotypes, thematic inventories, visual alphabets and dictionaries, mock manuals, juxtapositions of people, behavior, or objects for a humorous effect or the viewer's critical evaluation, and so on. In some exceptional cases, the strip design may also support a nonsequential series of images, such as when the images in a strip can be read in various orders, or need to be viewed from many directions. ${ }^{8}$

In terms of the image content, the sequential form can be affirmed in multiple ways, such as by the recurring character or an object, or the position, orientation, and gaze of the depicted figures. Other visual markers of sequence in the early nineteenth-century picture stories contain formal devices and instructions for the order of reading, including numbered panels, the placement of the captions or the speech balloons so as to facilitate the sequencing between the panels, and the use of frames to 
suggest an order of reading and viewing. By contrast, the impression of continuous motion or action is rare in this body of work. ${ }^{9}$

Why and how the sequential form acquires prominence in the course of the nineteenth century, and how it does become associated with the strip design, is a complex historical issue. To answer this question, we need to problematise, on the one hand, their historical relationship. On the other hand, we need to open up the question of the historically contingent models of visual narrative in this body of work, including especially questions of narrative organisation and form.

In his ground-breaking study The Early Comic Strip, which examines the prehistory of the comic strip from $c .1450$ until 1825, David Kunzle made the useful distinction between a picture story and a strip design, claiming that "all narrative strips are picture stories, although not all picture stories are, in the first instance, narrative strips" $(1973,5)$. The distinction allows that the strip design, while being "more or less narrative by definition", does not always function narratively, but may have other functions as well. The design can take, for instance, a descriptive function, such as the creation of a panoramic view of society or the depiction of types of people $(1973,5)$. Furthermore, the strip design is not the only compositional form that can serve narrative communication in the broadsheet format. For Kunzle, a 'narrative' in the art of broadsheet caricature accommodates both a sequence of separate images, in particular in the form of the strip design, and what he calls the "single-setting narrative" $(1973,4)$. The latter applies, more precisely, to engravings in which are depicted, within a single image, two or more episodes from the same story.

One-panel narrative cartoons were published in different formats before the emergence of the satirical magazines of the late 1820s or the illustrated magazines a decade later. In fact, we should specify that the single-setting narrative, in Kunzle's definition, has two quite distinct forms: the proper single-image narrative, which depicts some narrative sequence in one image, and the story told in a series of separate images collected in a narrative album in the Hogarthian style from A Harlot's Progress (1731) to The Four Stages of Cruelty (1751). At the same time, the relationship between a series of images in an album and the story told in the strip design is close, and Kunzle points out that their distinction is to some extent only a matter of presentation: any narrative album could be presented as a strip, and any strip can be mounted in an album $(1973,5)$.

Perhaps surprisingly, however, Kunzle has relatively little interest in the historical aspects of the narrative form and the way in which changes in the publication format contributed to the emergence of the early comic strip. The transition to the comic strip from the late eighteenth century to the mid and late nineteenth century involved a number of significant changes in the publication format, from narrative 
albums and broadsheet prints to caricature magazines and illustrated satirical magazines, then comic magazines and albums. These transformations were accompanied by changes in the way in which picture stories could be told. Narratologically relevant inquiries that could be pursued in this context involve, for instance, the development of techniques for creating narrative voice, perspective, characters, plot structure (time and causality), sense of action and movement, the setting, and narration through showing, style, and page layout. The development of the narrative use of the speech balloon has perhaps been given most attention in this regard. ${ }^{10}$

The rest of this chapter will discuss the available alternatives in the organisation of picture stories in the early nineteenth-century caricature and narrative drawings with a specific emphasis on the narrative uses of the multi-panel sequence in the satirical caricature magazines published between 1825 and 1835 . The main examples are taken from caricature magazines published in Britain and Scotland. These include, in particular, the fortnightly Glasgow Looking Glass (1825), today considered by some as the earliest comics magazine, and which changed its name to Northern Looking-Glass after five issues, to run for nineteen instalments altogether (1825-1826); the continuation of this publication in the monthly The Looking Glass or, Caricature Annual (1830-1836), based in London and also known as McLean's Monthly Sheet of Caricatures, or The Looking Glass; and the caricaturist Charles Jameson Grant's fortnightly single-page broadsheet 'magazine', Every Body's Album and Caricature Magazine (1834-1835), that ran for a total of thirty-nine issues. ${ }^{11}$ The principal illustrator of Glasgow/Northern Looking Glass and the first issue of The Looking Glass was the English artist William Heath (1794-1840). Robert Seymour (1798-1836), who is best known for his illustrations for Charles Dickens' novels, continued Heath's work from the eighth instalment of The Looking Glass onwards. Unlike the weekly illustrated magazines, such as Figaro in London (1831-1839), for which both William Heath (under the pseudonym "Paul Pry") and Robert Seymour contributed, or the more successful Punch and The Illustrated London News that started in the early 1840s, these caricature magazines mainly featured cartoons and picture stories. ${ }^{12}$ In the course of this discussion, I will make comparisons between this body of work and illustrative examples of the British Golden Period of caricature, roughly extending from 1780 to 1820 , in order to be able to better contextualize the forms of narrative organisation and the graphic innovations in the satirical magazines. These Golden Period works will include selected narrative drawings, published as broadsheet prints, by James Gillray (1756-1815), Richard Newton (1777-1798), George Woodward (1760?-1809), Charles Williams (active 1797-1830), and George Cruikshank (1792-1878). 


\section{Narrative Form and Publication Format}

\section{Principles of Narrative Organisation}

The early nineteenth-century picture stories in the satirical illustrated magazines can be conceived around three operative principles of juxtaposition, sequentiality, and simultaneity. These principles of narrative organisation are grounded in the history of caricature and the illustrated press, but should be seen as heuristic devices rather than as historical categories. They are not mutually exclusive, but can be combined over a scale of variation where most cases would have to be characterized as intermediate. In fact, pure instances of these principles are rare.

\section{Juxtaposition}

The juxtaposition of two or several images, parts of one image, or text and image, is widely used as a means of narrative organisation and visual communication in this body of work: juxtaposition can provoke meanings through contrast, association, extension, similarity, difference, reversal, and antithesis, among other things. It can be used as a technique in a single image as well, between two images, or a series of images. The effect of juxtaposition may in some cases also extend over several images in a long strip or between two or more strips.

Humorous contrast is perhaps the most common manifestation of this principle in the satirical prints and caricature magazines, based both on the visual contents of the comparison and the contrast between the caption and the images. We can observe this in the broadsheet caricatures, for instance, when a delightful young woman with a slim figure, a "nobody", is contrasted with a buxom lady, who is somebody important (Richard Newton's "No Body Some Body", 1795); when a moonstruck young man who looks at a miniature of his beloved is juxtaposed with a corpulent old wine lover, who is angered by a new wine tax (Richard Newton's “Contrasted Lovers”, 1796); or when four different nationalities (the English, the Scots, the French, and the Dutch) are contrasted through their "civilized" and "primitive" lavatorial habits (James Gillray: "National Conveniences", 1796). In the first issue of The Looking-Glass (1830), in William Heath's "Siamese youths, our own youths", Siamese twins, conjoined by their bodies, are compared to "the British youths", Duke of Wellington and Sir Robert Peel, the long-time close political allies. The titles of these works confirm the sense of contrast, or similarity with a significant difference, such as physical figure, cultural context, social standing, between the figures thus juxtaposed.

The many narrative uses of juxtaposition in these works include, especially, contrasted perspectival changes, speech and dialogue, or temporal framework ("before" and "after", "then" and "now"), and certain devices of spatial division that call for a narrative response. For instance, changes of perspective, such as shifts between close-up and distance, or between different points of view on the same situation, can 
suggest a magnification of the situation or an effect of accumulation. William Heath parodies the English actor Edmund Kean (1787-1833) in the two-part caricature entitled "Kean in America", published in December 1825 in Northern Looking-Glass, by juxtaposing two images of $\mathrm{Mr}$ Keen performing Shakespeare for American audiences. In these images we can read Kean citing lines from Richard III and Richard II while he is greeted with insults from the audience, who consists mostly of African-Americans. In the first image, round objects, sticks, and what seems to be a dead cat are thrown onto him on the stage. In the second image, we then see Kean much closer, citing Richard II in the same costume, but this time he no longer appears to be on the stage, but pleading in a street with a copy of Thomas Moss's "Beggar's Petition" in front of him. The two African-Americans who pass and speak stereotypically broken English recognize the actor, calling him "a very bad man" and "a naughty man". The changing perspective and setting, as well as the dialogue in the speech balloons, make a satirical point, but also describe a dramatic situation. Thus, this two-part image has the potential to be conceived both as a deepening of the satirical take on the same situation-Keen's humiliation in front of the American audiences-by means of juxtaposition, and as a sequence that shows how his situation is deteriorating. Our choice, whether to regard this as a narrative sequence or not, would partly depend on how much emphasis we are ready to put on the temporal and causal connections between the two panels. The juxtaposition itself has weak narrativity.

Dialogue, in the literal sense of a conversational exchange, can strengthen the sense of duration between the images and elements in juxtaposition. The two panels in George Cruikshank and the anonymous artist J. Pxxxy's broadsheet print entitled "Back \& Front View of the Ladies Fancy-Man, Paddy Carey O'Killus” (1822) feature extended dialogue in speech balloons between the spectators gathered around the newly erected Duke of Wellington statue in Hyde Park shown from two juxtaposed angles. The statue, where Wellington is allegorized as Achilles, was London's first nude sculpture, albeit garnished with a fig-leaf, and is shown here to be an intense object of curiosity and looking. One of the spectators uses a lorgnette to better see the details, many people point at the statue, even a telescope is directed at the monument, and the spectators utter excited comments on both sides of the statue (Figure 10.1). The first panel shows the statue from the back and the second from the front; the two panels are divided by a gutter. Most spectators who crowd around the monument are women, with the exception of Wellington himself and two children. Wellington is caricatured in the first image in uniform looking at the statue in profile and saying: "The Honor is so great, that all I can say by the Powers, is that I'm Speechless". The dominant role of women in these images is a reference to the fact that the statue was funded by subscriptions from women in honour of the Duke's services for the nation. 


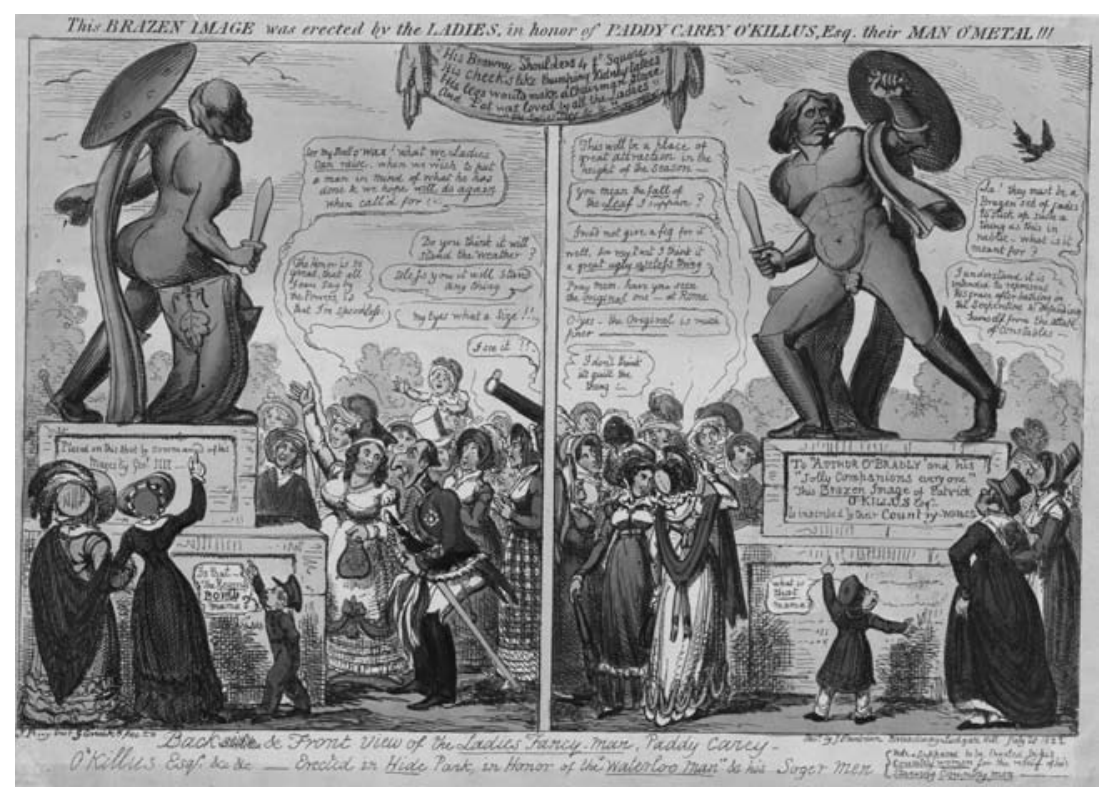

Figure 10.1 George Cruikshank and J. Pxxxy's "Back \& Front View of the Ladies Fancy-Man, Paddy Carey O'Killus” (1822). Courtesy of The Lewis Walpole Library, Yale University.

The spectators featured in the juxtaposed panels are not the same, but form similar groups around the statue. This could suggest that the perspectives represent two distinct but close moments. Some of the utterances are spontaneous individual remarks, such as the comment "I see it!!", which seems to come from the person who is shown to use telescope to better grasp all the details of the statue. However, most of these utterances involve an exchange of words, such as the question of the fig-leaf that extends to a longer conversation in speech balloons: "This will be a place of great attraction in the hight of the Season/You mean the fall of the Leaf I suppose?/I would not give a fig for it/Well, for my Part I think it a great ugly useless thing/Pray men, have you seen the Original oneat Rome/O'yes-the Original is much finer/I don't think its quite the thing" (underlining in the original for emphasis). The situation is thus extensively satirised by means of images and dialogue alike from two juxtaposed angles. Nevertheless, this is not a narrative in a literal sense, for the lack of temporal development and causal connection between the panels, but represents a situation with much narrative potential.

The juxtaposition between two or more images or elements in one image can attain a stronger sense of narrativity whenever it is supplemented by some causal connection or a sense of development in a continuing 
temporal frame. This can occur, especially, when juxtaposition takes the rudimentary narrative form of two or more polarised moments and situations in comparison. ${ }^{13}$ For instance, William Heath's picture story "Politics" from Northern Looking-Glass (18 August 1825) depicts, in two vertical strips of four panels, the daily habits of the King of France at his castle from the morning, when the newspapers are read to him, until the evening when he is shown to go to bed. ${ }^{14}$ There is no causal link or sense of continuity between the depicted actions. These are just typical events-breakfasting, going to mass, shooting sparrows in the park, and playing with the dogs and grandchildren-during the King's typical day. Is this picture story, then, based on a juxtaposition of situations or a sequence of episodes? The temporal and spatial frame gives the panels a sense of sequential order. There are, also, some thematic and visual correspondances across the two strips, based on the structure of juxtaposition, and that potentially emphasise the sense of repetition. For instance, the last panels of each strip show the King lying down, "reclining" on his bed during the day and going to bed at the end of the day. Juxtaposition thus connects the sense of temporal progress with cyclical structure: events are in sequence, within the temporal frame of 24 hours, but also contrasted and connected across the chronological order.

A more extensive version of a similar arrangement can be found in Charles Williams's broadsheet "The Two Journals" (1814) that contrasts, in two juxtaposed two-tier strips with eight panels, the daily life of two royal figures: the virtuous and studious Tsar Alexander, who is visiting England and spending a day in London in the most beneficial way (taking a walk to Kensington, taking notes of a military drill, writing a letter to his wife, etc.), and the idle and vain Prince of Wales who spends the day at his palace. In contrast to William Heath's depiction of the daily chores of the French King, we can observe here a clearly continuing and causally motivated string of events that unfolds from one panel to another. The sense of narrative sequence is achieved both on the level of showing that describes connected phases of one event and, perhaps more significantly, by means of first-person verbal narration that provides the story with bridges between the panels. For instance, in the first panel of the Prince of Wales's strip journal, we see the Regent in the bed and can read in the accompanying caption that "Boozy and sick, with aching head,/Toss'd sleepless, on my swan down bed./Sunk tow'rds morning in a dose, /When dreams of frightfull import rose". The next panel shows the Regent still in the bed, uneasily asleep, and having a vision of a demon that his wife wards off. In the third panel, there are three valets working on the Regent's hair or wig. The situation has changed, but the caption, or the citation from his journal, explains what has happened: the Regent has finally woken up at noon and is now having his hair done (and this will last for 2 hours). Similarly, the following panels suggest narratively cohesive structure where both what is verbally 


\section{6}

narrated and what is shown in the images can be followed as a sequence. As a whole, however, the satirical point of the composition is based on the juxtaposition between the two royal figures and their contrasting habits and characteristics. ${ }^{15}$

Juxtaposition can also be incorporated within the genre of a 'progress' story in a series of images, made famous by Hogarth's prints that contrast various phases of a person's life, typically reflecting the character's career and moral development. The progress story continues to be varied throughout the Golden Period of Caricature in the works of James Gillray, John Nixon, Richard Newton, and George Cruikshank, and equally, William Heath makes various versions of it in the caricature magazines. In this genre, juxtaposition becomes an element of the sequential form. For instance, a temporal and causal sequence can be used to make a satirical point by means of contrast, or a series of events may transform into a structure of juxtaposition between two or more ideas, themes, or perspectives. William Heath's picture stories "Essay on Modern Medical Education", from Northern Looking-Glass (Vol. 1, No. 6), and "Life of a Soldier" (Vol. 1, Nos 10-16) are examples of such shifting emphasis. In "Essay on Modern Medical Education", which is a series of ten panels satirising modern medical education, some of the panels suggest a sense of temporal progression and narrative sequence, while elsewhere the sense of sequence is weak or nonexistent. The first three images in this series do not imply a narrative logic as a sequence, but function as distinct caricatures. They depict a mob protesting the grave robbers who dug up bodies to sell them to anatomists at the medical schools ("The alarm, or the Kirk Yard in danger"), drunken behaviour at a funeral club ("The dead association") and a meeting to counteract bodysnatching ("Watching and warding"). The following second instalment with three panels, however, suggests some degree of temporal progress. All of these images follow student life at a medical institution, from an anatomy lecture, where the stolen body parts were dissected, to pranks in an apothecary's shop, and the graduation ceremony.

The numbering of the panels from one to ten, and the use of the phrase "to be continued" at the end of the second instalment, obviously indicate that the story will continue, and the mention of "continued" in the beginning of the last instalment, further emphasise the fact. Yet, it is important to note that the last four panels that illustrate practising doctors at work are organised in two tiers of two panels based on a satirical contrast rather than temporal development or narrative sequence. The captions of the images present the panels in pairs of two. The practicing of vivisection on animals ("Preparing for practice") is contrasted with the amputation of a man's leg with an axe ("Actual practice"), whereas the poor hospital conditions of home ("At home"), where skeletons are seen to tend the sick and dying, are juxtaposed with the terrible 
circumstances of a battle field, where surgeons attempt to reassemble the mutilated soldiers' severed legs and head ("abroad").

However, the polarised structure of 'before' and 'after', or the contrasting of events within some other temporal frame such as 'then' and 'now', do not always serve a narrative function. Such temporal structures can also make a statement, that is, have an argumentative function. For instance, James Gillray's broadsheet image "The Blessings of Peace, the Curses of War" (1795), which is dedicated to the Parliament of the Great Britain, contrasts, in two roundels or medallions, an image of peace and war to make an anti-war statement. Here, the first image shows happy family life in a prosperous British home with the caption "such Britain was!", while the the second image portrays a family outside a destroyed farmhouse lamenting the death of the father (or husband), who is stabbed to death with a bayonet somewhere in the warring Europe, with the caption "such Flanders, Spain, Holland, now is!". The topical contrast between "Blessings of peace $\&$ prosperity and domestickhappiness" and "The curses of War, invasion, massacre \& desolation" is also accompanied by the inscription, placed in between the upper parts of the roundels, "from such reverse O GRACIOUS GOD, preserve Our Country!!!". The inscription specifies the purpose of the comparison by giving this contrast a logic of an argument: the possibility of a war at home that needs to be prevented, and the threat of a French invasion that needs to be addressed. Similarly, in George Cruikshank's caricature "John Bull's Three Stages or, From Good to bad \& From Bad to Worse", published in the political monthly The Scourge (1811-1816) on 1 March 1815, the contrasted situations in the life of John Bull and his family make an argument against war. In this folded colour strip, the three panels that are entitled "Before the war", "During the war", and "Peace with all the world" show first how war deprives the family of the necessities of life, then changes their physical appearance from rosy-cheeked, robust, and cheery to a starved and miserable state, and finally how the following peace only worsens their situation. John Bull and his family eat a large meal before the war, much less during the conflict, and have only bones on their plates when the peace arrives.

In some broadsheet caricatures as well as narrative drawings in the satirical magazines, the structure of juxtaposition can emphasise the spatial division of the image that reflects two neatly distinct spheres or realms of life. On the one hand, we can observe this in images where some aspect of the image suggest an inner division and thus potentially undermines the distinction between a single image and two panels. For instance, in James Gillray's caricature entitled “BLACK-DICK turn'd Taylor" (1788) a tailor's shop-board suggests a division of the image field into two relatively independent visual spaces. In the upper part we can see Admiral Richard Howe, known to his men as "Black Dick", sitting on a tailor's board and working on a naval coat. In a speech balloon, 
the Admiral explains his plans for reform-new uniforms, ships, appointing young (unexperienced) officiers and admirals. In the lower section of the image, a demon echoes the Admiral's words and reveals his own plan to control the Admiral's reform ("I shall get you before you are aware of it"). The Admiral is not aware of the demons working underneath him, but the demons can see and hear the Admiral from below. Similarly, in one of Charles Jameson Grant's several cartoons in the 15 September 1834 issue of Every Body's Album that focus on the theme of angling, the water line serves as a virtual frame inside the image dividing it into two sections. Above the water line, a fisherman sits waiting with his hook and line in the water, while below the water line two fish, unseen by the fisherman, converse and mock the man and his methods in their own virtual panel (Figure 10.2).

On the other hand, the juxtaposition of elements can contribute to spatial division in the embedding. For instance, in Gillray's caricature "Hope" (1802) we perceive, in the left side of the image, the symbolic figure of John Bull standing in the lobby outside the House of Commons, voicing his concerns about the economical situation and the budget plans, while through the open door on his right we see Prime Minister William Addington making a budget speech inside the House of Commons. John Bull's rumination and comments ("Let me see-25 millions. How are we ruined? Income Tax taken off! Well! Well! Well!”), concerning Addington's abolishment of the unpopular wartime income tax, function as a means of connection between the embedding and embedded image. The two men, furthermore, are associated and at once contrasted by their positions and physical shape. They both stand, in profile to the right, with one leg forward, within the same perspectival line, while the speech balloon, superimposed on the embedded image, directs our gaze to Addington in the House of Commons. ${ }^{16}$

In yet other kinds of broadsheet caricatures, certain visual means of connectivity, such as speech balloons, lines of sight and perspective, and shared background space, can create thematic, conceptual or scenic connections between juxtaposed images without a particular narrative function. For instance, the positioning of the speech balloons and the shared background in James Gillray's satirical print "Nelson's Victory, or, Good-News Operating Upon Loyal Feelings" (1798) forges connections between different reactions of desperation and disappointment by the various Members of the Opposition when they have received the news of Horatio Nelson's victory at The Battle of the Nile. In Richard Newton's "Sketches in a Shaving Shop" (1794), which depicts humorous scenes at a barber shop, some of the twelve featured barbers' utterances or lines of sight, which point outside the panel frames, indicate that there is a larger social situation taking place at the barber shop beyond what is shown in the image. In these juxtapositions of similar situations, the juxtaposed elements may offer an incentive for further interpretation, 


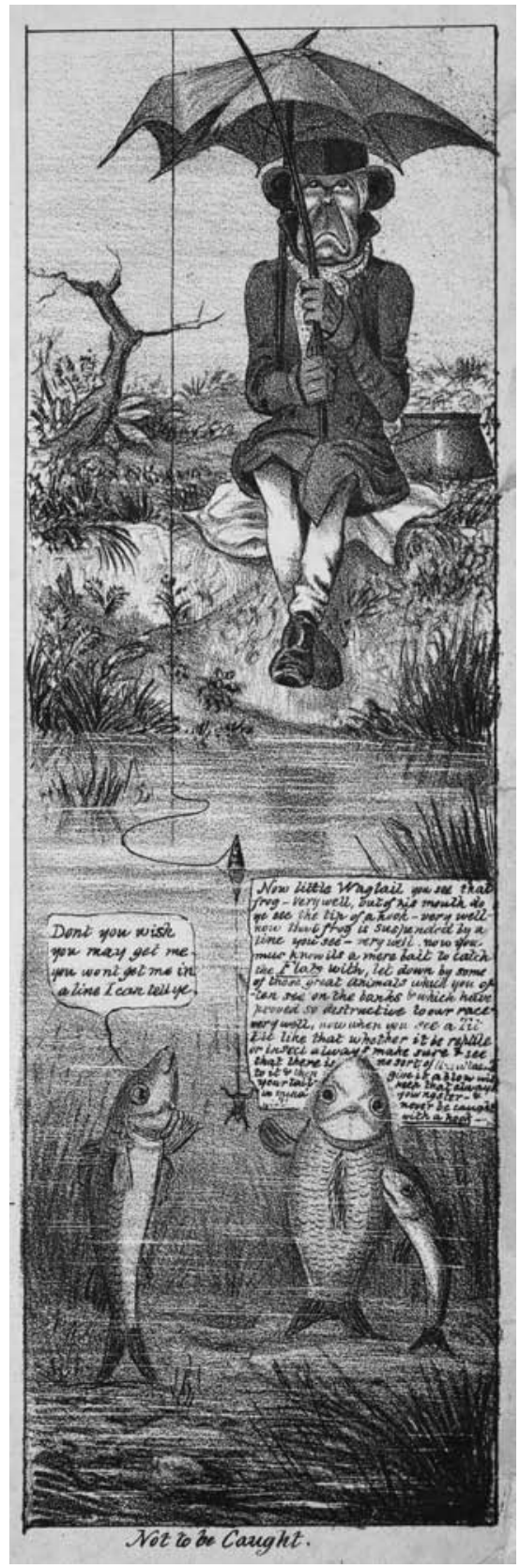

Figure 10.2 Charles Jameson Grant. Every Body's Album 15 September 1834. (C) The British Library Board, LOU.LON 1052. 
i.e. prompt the viewers to think how the things are related. Juxtaposition, then, functions as a question, a joke, or a kind of enigma rather than a narrative.

\section{Sequentiality}

In the historical context of the early nineteenth-century British satirical magazines, the sequential form of narrative organisation operates on a continuum of various kinds of temporal and causal relations. In this regard, we can distinguish two basic forms of sequentiality: the episodic sequential form and the narrative continuity sequence. Furthermore, the sequential form of these picture stories can interact dynamically with forms of organisation and layout that are based on juxtaposition and meaningful contrast, or the sense of simultaneity between the images that invites a more global look at the composition.

The episodic sequential organisation involves two or more images in series where each image represents a stage or phase of a situation or an event. The episodic picture stories in the caricature magazines and broadsheets of 1825-1835 exhibit considerable variety in this regard, and the balance between episodic and more continuous arrangement can also shift in the course of the story. At one extreme, these publications feature picture stories where an episode in a single image is more or less self-contained and, consequently, the order of the episodes may be rearranged without affecting the work as a whole. In these cases, the logic of the sequence is based on a recurring character, place, or theme; the causal connection between the visual contents, actions, or speech situations included in the images remains weak. At the other extreme, episodic picture stories can include, both in the images and the texts, strong markers of causal development that necessitate that the sequence is read in a certain order. The genre of 'progresses' is the most common genre in this kind of episodic arrangement. In the 'progress' story, the passing of time between one image and another can usually be observed in the main character's changing physique and social standing.

We can count at least three picture stories as clear cases of the progress genre in Northern Looking Glass: "Life of a Soldier", "Life of a Sailor", and "Life of an Actress". While the principle of juxtaposition operates in these stories as well, it is overshadowed by sequential structure. The longest of these, "Life of a Soldier" ${ }^{17}$ which consists of twelve numbered cartoons (in our modern sense of the term) in several instalments, describes a soldier's career. The contents of the images reflect the idea of significant episodes in a developing career: the first three panels portray the soldiers' training and promotion, the next pair of panels show the embarkation and the ensuing battle, and the following pair of images depict a fight between a British soldier on the ground and someone elsethe opponent is dressed in what may be an Arab costume-and then 
shows a wounded soldier walking on crutches. The numbering of the panels from one to twelve, and the use of the phrases "to be continued" and "Cont'd", even if this is not altogether systematic, give indications of the order of reading. The first instalment in issue 10 (14 November 1825) has three panels, the first of these folding out from the middle of the page to extend the width of one panel. We need to note in this respect that panels numbered 5 and 6 (third instalment, "The Embarkation"/"The Action") are curiously printed in the reverse order from right to left: the panel on the left is accompanied with the mention "to be continued" (Figure 10.3). While this is likely to be a mistake in printing, we can also speculate that another reason for the numbering of the panels and the use of the phrase "to be continued" might be to guarantee that all readers can understand how these narrative drawings are set in sequence. ${ }^{18}$

Yet, some features of "Life of a Soldier" also suggest that the sense of narrative sequence is quite weak or ambivalent in this case. On the level of the image content, the instalments show various situations in a soldier's career, from hiring a new conscript to becoming an adjutant. One challenge in conceiving these images in terms of a continuing narrative, at least in the first instalments, is the protagonist's somewhat uncertain visual identity. In fact, given that the soldiers in each panel look somewhat different, can have their backs turned towards the viewer, or wear hats that cover parts of their face, it is possible to read "Life of a Soldier" alternatively as a story of an individual British soldier or as a story of unidentified conscripts who represent typical situations in the course of a military career. That said, however, the accompanying written explanations of the story in the issues of 12 and 13 of Northern Looking Glass (12 and 26 December 1825), after the narrative had run for four panels in two previous issues, make it evident that the story is anchored on one particular British soldier.

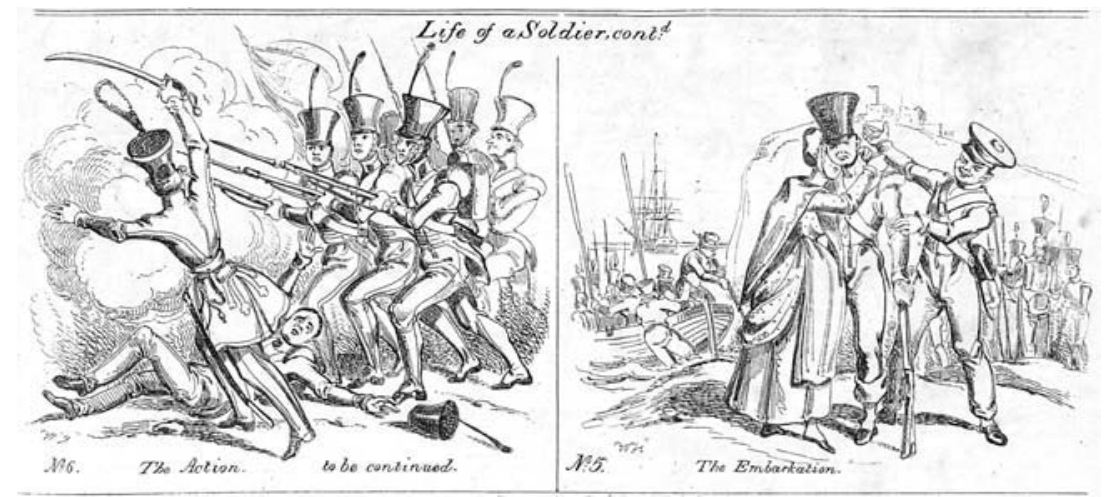

Figure 10.3 William Heath. "Life of a Soldier". Northern Looking Glass XII. 12 December 1825. (C) The British Library Board, P.P.6223.dba. 


\section{2}

The episodic situations in this story are linked thematically within the biographical frame of the soldier's career. Within each instalment, moreover, the images have more or less evident causal relations with each other. For instance, the scene of embarking on a navy ship is followed by a battle scene; an image showing a fallen soldier fighting on the ground is followed by an image of a wounded soldier on crutches; and after a British soldier is seen struggling to seize a French eagle from a French soldier, he is, then, having captured the eagle, being presented to the Duke of Wellington (panels 9 and 10).

In contrast to the initially loose but gradually more coherent narrative sequence in "Life of a Soldier", William Heath's "Life of an Actress" (Northern Looking Glass, Vol. 1, No. X, 14 November 1825) is an imaginative take on the progress genre where the eight images of the strip create a temporally and causally tight and coherent sequence. The strip design has also been put to an expressive use: the ascending ladder frame of the strip, with one panel 'placed' on each broadening step, accompanied by the gradual increase in the size of the panels, provide a clear symbolic frame for the actress's gradual climb on the social ladder (Figure 10.4). At the same time, the steps of the rustic ladder indicate the order of viewing the images. The ascending viewing protocol, thus, echoes the actress's societal climb from the poverty of her home to the fame of the stage, and through widowhood and wealth to a noble status. Moreover, there is considerable narrative coherence in the image content throughout the sequence: the heroine and the signs of her increasing wealth, social standing, and growing corpulence are identifiable in each panel. The contemporary readers were likely to recognize in this depiction the popular actress Harriot Mellon (1775-1837) who, born in a family of strolling players in a travelling theatre company, became, successively, the wealthy widow of a London banker and the Duchess of St. Albans.

As "Life of an Actress" goes to show, the progress genre can be realised in the strip design or, alternately, the strip design can accommodate the progress story. ${ }^{19}$ In "Life of an Actress" we must note, moreover, how the design of the strip supports, ironically, the idea of progress as a form of social climbing. The order of the reading is significant, both for the theme of the strip, but also from our historical perspective concerning the development of narrative forms. "Life of an Actress", with panels to be read from the low right-hand corner of the strip upwards to the left, illustrates how the idea of sequence is quite flexible with regard to the order of reading in this context.

What makes some narrative a comic strip, in the sense that we understand this form of expression today, instead of the episodic picture story? As we have seen, the progress story and its variations are based on thematic, temporal, and causal relations, often in the sense of juxtaposition, between the contents of a series of images. Narratives in the strip design 


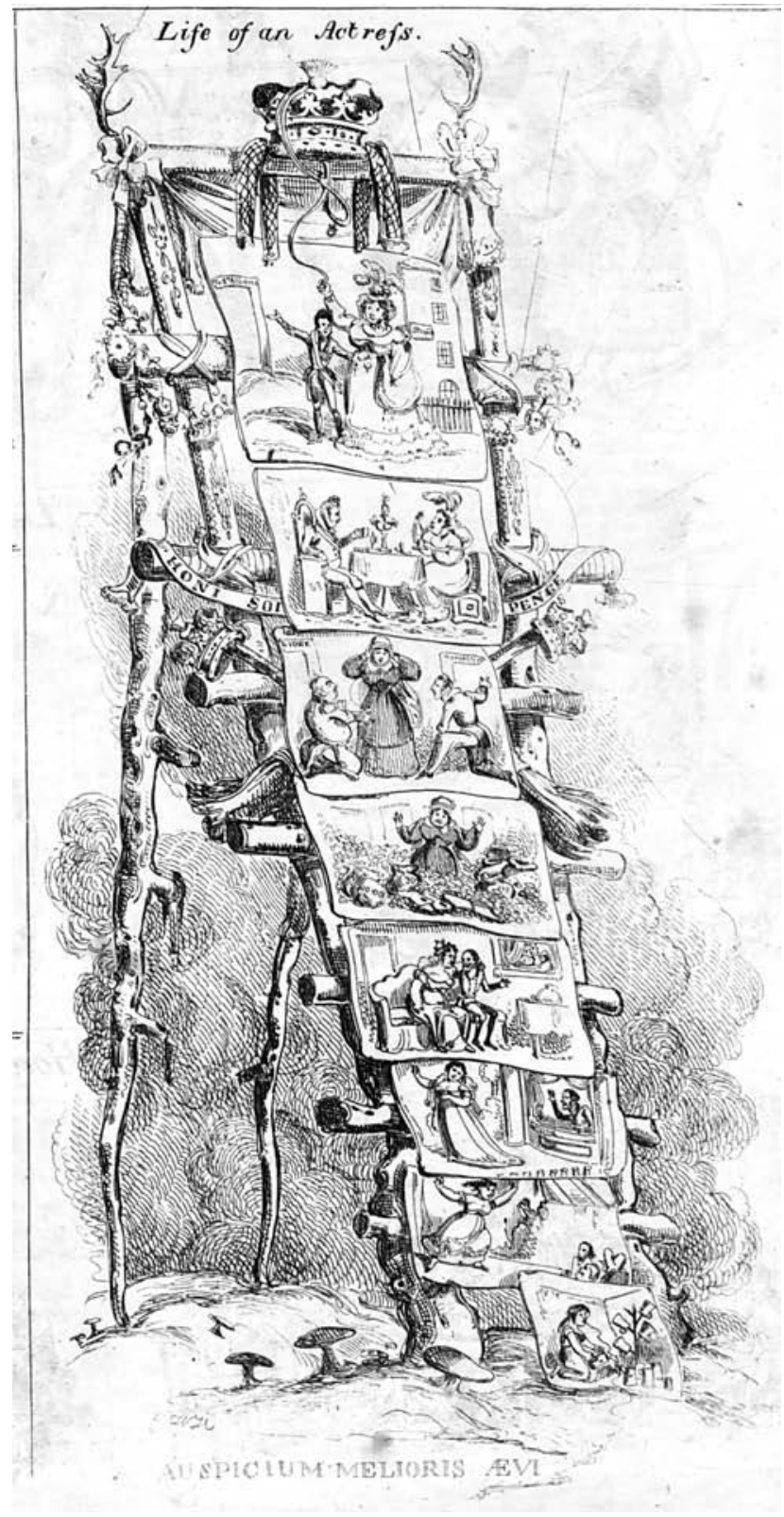

Figure 10.4 William Heath. William Heath. "Life of an Actress". Northern Looking Glass X. 14 November 1825. (C) The British Library Board, P.P.6223.dba. 


\section{Narrative Form and Publication Format}

in these same publications can follow a similar logic. The difference between these two, then, is the kinds of thematic, temporal, or causal links that can be recognised between the images. The narrative use of the strip design creates the impression of a sequenced, rather than juxtaposed, event, experience, or situation, and of continuity rather than comparison. Typically, the logic of narrative sequence predominates also on the level of the image content; it is not just implied by the juxtaposition of phases in some temporally structured development or sense of progress.

However, in some cases, the progress story can suggest temporal and causal relations (consequence) between images in ways that move the genre close to a narrative comic strip. For instance, David Kunzle notes that in John Nixon's picture story "Progress of Passion" (1792, etched by Isaac Cruikshank), the narration of the continuous displacement of an emotional reaction ditinguishes this work from most previous progress stories (Kunzle 1973, 363). Nixon's two-tier strip print shows a sequence of nine scenes starting, in upper left, with King George III dismissing the Tory politician and Chancellor Edward Thurlow, followed by a chain of events resulting from Thurlow's anger that affects his entire family and household, servants, the butcher, a dog, and finally a cat that kills a mouse. Thus, we can follow a chain of causally linked events from one panel to another. What is seen in one image explains what has been seen before, but also points to what comes after. In each scene of this cycle of abuse, the figure who has suffered from someone's anger, scolding, or physical attack, is consequently shown to perpetuate the abuse to others. Thus, on the one hand, passion "progresses" downward in terms of the figures' social status while, on the other hand, the genre of the progress story, based on comparisons between stages of the process that invite careful study of the occurred changes, is transformed into a closely tied chain of events.

We need to illustrate the crucial point about sequenced rather than juxtaposed structure since the distinction between the episodic and the narrative sequence can remain rather indeterminate. Characteristically, in the caricature magazines of the 1820s and the 1830s, when the strip design is used to deliver a narrative sequence, we can observe a sense of temporal and causal continuity on the level of the image content, as well as between image and text, and not just within the general temporal frame of a 'progress' or a 'life'. This entails, furthermore, that the temporal gaps between the images are sufficiently close to maintain a sense of continuity instead of mere juxtaposition, contrast, or comparison. What is sufficiently close in this context is, of course, relative to the kind of story and its representation of time; and what one reader might perceive as narrative continuity someone else may perhaps experience as discontinuous. Yet, some distinctions can be made.

We can observe the operation of narrative continuity sequence, for instance, in the first sequential comic strip in Glasgow/Northern Looking 
Glass, "The History of a Coat", which ran from issues 4 to 6 between 23 July and 18 August 1825. The story was published in three instalments, respectively, with four, three, and two pairs of panels in two vertical columns. The first instalment does not have captions. The layout is not without ambivalence in the sense of sequence: the order of reading the strip design is not quite obvious from the outset due to the fact that the two rows of panels are separated by a full line (or frame) vertically, and a partial line horizontally. This could suggest, for instance, that the sequence is meant to be read from top to bottom. The content of the images, nevertheless, clearly indicates that the story of the coat evolves horizontally. In the second and the third instalments, the order of reading is further confirmed by the captions. In the images, we can follow the gradual making of the coat, from the shearing of a sheep to the loom and the tailor's table, then the slow degradation of the cloth-in the true spirit of the 'progress' story-from a gentleman's coat to a scarecrow's wooden shoulders and, finally, a dirty cloth torn by ragged pigs in a panel with a caption "End of the Tail".

The strip has the basic features of a moral life story, exactly as in the progress genre, but the difference, from the contrasted phases of a 'progress' story, is the greater reliance on the sense of consequence between the panels and the need to work out their relations in the story. In other words, the impression of temporal and causal continuity between the panels is more crucial here than in the Hogarthian progress stories that revolve around the principle of comparison between the phases of a life or a career.

Another indication of how the sequential form moves closer to the narrative use of the strip design is awareness of the gap between the panels as a form of temporal or logical discontinuity that needs to be bridged and explained. One illustrative case of an episodic picture story turning more strip-like in this sense, based on quite literal awareness of the panel relation as a meaningful gap of information, can be found in a picture story entitled "Scenes from a Historical Drama" from The Looking Glass (No. 36, 1 December 1832). The story describes the history of Belgium and Holland in six panels where the first three images, referred to as the "first act", follow an episodic logic, depicting respectively the French conquests in the Netherlands in 1795, the liberation of Holland in 1813-1814, and the enlargement of Holland at the Congress of Vienna 1814-1815 to include what in 1830 became modern Belgium. Importantly, there is an inscription (sideways) in the blank space between the third and the fourth panels: "Here is supposed to occur the space of Sixteen years from 1815 to 1831 ". The space between the panels is thus perceived as a dramatic 'intermission', while it also marks a shift into a faster rhythm and more closely tied sequential structure. The gap of 16 years is followed by three panels that describe, in a faster succession of events and with several recurring characters (Belga for Belgium, Leopold I, 
and William I), the developments of the last 2 years: the Belgian revolution, the installation of Leopold I as the King of the Belgians, and King William's subsequent military attempt to reconquer Belgium. The theatrical metaphor of this historical chain of events is extended to the very end of the sequence where we can read: "We know not the denouement of the piece, but look for poetical justice".

The use of reoccurring characters in continuing action or situation distances the strip design from the episodic structure. Charles Jameson Grant's strip "The Adventures of the Buggings's", published in Every Body's Album (No. 14, 15 July 1834), which tells the family Buggings' mishaps on a journey to Gravesend and back in nine panels, affirms the sequential form at various levels simultaneously. Here we can follow the continuing group of characters and their movement, observe the temporal connections and shared framework between the panels (a day's journey from morning until night), and read the captions and the speech balloons that make various relevant causal links between the panels. In the morning, the taxman Buggings' family of six starts off slowly after having enjoyed twenty-four rolls and tea. Running late from their steamer, they hurry to catch boat on a bark, then sleep over the stop in Gravesend and end up in Margate where, just before landing, they all fall into the sea after their son, Bob, who had been looking for eels in the water. Finally, they return home in a Margate coach at night, all wet from their bath and the pouring rain, only to find their house destroyed in fire. The disaster, as it is ironically explained, thus forms "a kind of Consumation to their day's pleasure". In the last panel, Mr Buggings reassures his wife that he will make up for all the losses, in the manner of all great men, by a few "false accounts".

Still other means of narrative continuity involve causal connections through juxtaposed perspectives and voices. In the five-panel narrative entitled "Colonial Slavery" from The Looking Glass (No. 8, 1 August 1830), the narrative use of the strip design is not based on the process of following a recurring character through the strip, but on making causal connections between the depicted events, situations, and their speakers. The comic involves the real story of a female slave, so-called Poor Black Kate, who died of her owners' brutal treatment in the Bahamas in 1826. In the first panel, the slave owner named Mr Henry Moss of Crooked Island is threatening to flog the slave, who lies in a plantation field, while the woman's companion, a male slave, explains that she's already dead. The second panel portrays the slave owner, standing on what seems to be a terrace of his house, justifying his brutality. He is accompanied by his wife, while a black body can be seen lying in the background. It is noteworthy that in this panel, the mean-looking colonial couple has turned towards the reader. The man, looking directly at the reader of the strip, says: "Wot are you stareing at? Shant a man do as he likes with his own Ax. Your Duke of N-C-L". The reader is thus called upon to evaluate the 
slaver's actions and the justification of his claim (Figure 10.5). The three smaller panels in the lower row of the strip portray a chain of political reactions following the event. First, a colonial governor (Lt-General Sir Lewis Grant) is shown to appeal to the Members of Parliament on behalf of the slave owner, and then a Member of Parliament (Sir George Murray, Colonial Secretary) comments on the event on behalf of the slave owner and approvingly of the Governor's stance. Finally, the last panel shows two electors in conversation, reacting to the event. One of them is crying out "MURDER!!!", while the other wonders: "What 'sinnifies' It's My Intrest".

The verbal narration in this strip, involving both the captions and the reported speech of the characters, is crucial in creating narrative coherence between the five panels. The captions identify the main characters and the chain of events: "THE SLAVE", "THE PLANTER who Murderd the Slave!!!”, “GOVENOR sorry to punish the murdrers!!!”, "The MPs who approve of the GOVENOR", and "the ELECTORS who return the MP!!!”. Beyond the captions, all panels include the characters' viewpoints, which are given either in speech balloons or in quoted writing (the Governor is seen writing a text). These viewpoints showcase governmental hypocrisy in the matter and provoke the reader to evaluate the justice or, perhaps rather, injustice, done. Thus, the strip actively prompts the reader to make connections between the various scenes and

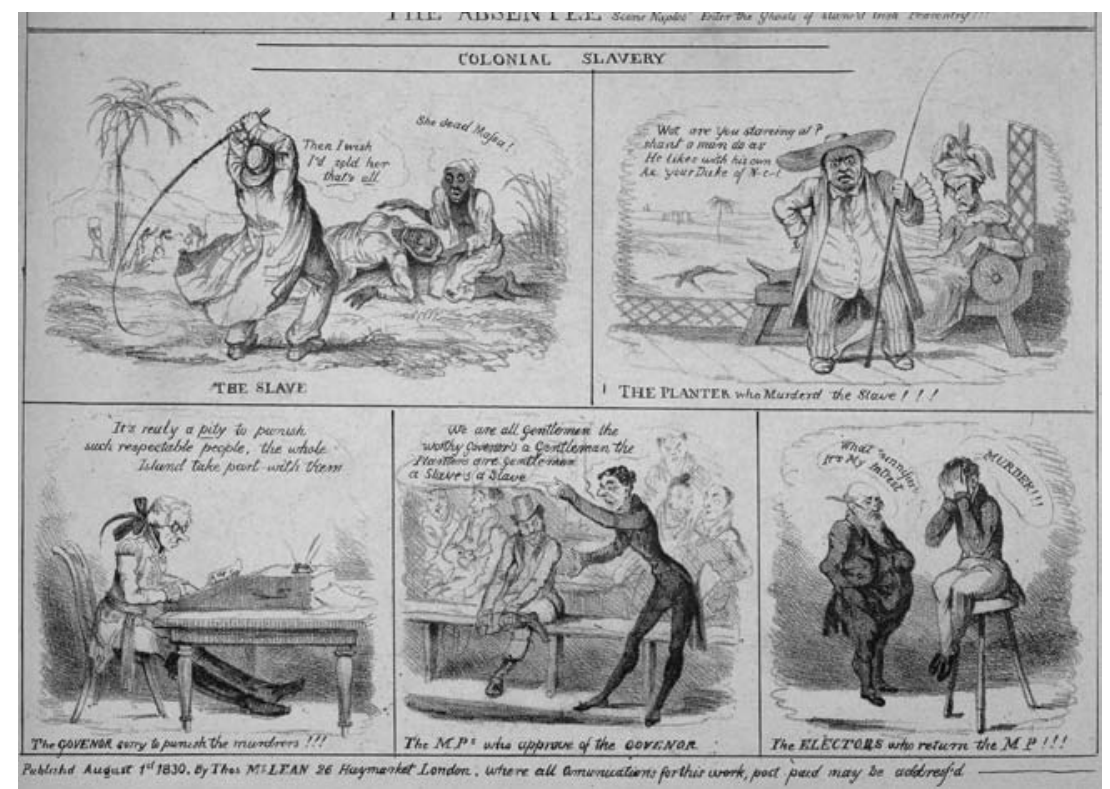

Figure 10.5 Robert Seymour. "Colonial Slavery". The Looking Glass, No. 8, 1 August 1830. (C) The British Library Board, RB.31.c.31. 


\section{8}

situations, not just in order to undertand the chain of events, but to perceive the various political stances in the case and take a stand of one's own as well. ${ }^{20}$ This strip is, then, a narrative representation of a chain of events and positions that portray how the judicial and political system attempts to justify the system of slavery.

\section{Simultaneity}

As we have already seen, the strip design can be employed for other than narrative purposes. This can involve, for instance, description, argument, or the development of a theme. In strips caricaturing the same character in different situations or sketching different types of people, the strip design can serve the purpose of inventory and accumulation rather than narrative sequence. The descriptive use of the strip form may also follow a spatial logic. In William Heath's full-page drawing "My House in Town" in Northern Looking Glass (Vol. 1, No. XV, 23 January 1826), the twenty picture panels of this work are set in eight rows, representing an interior of a room enclosed in a town house from the coal and wine cellars to the garret. There is no sense of narrative sequence between the panels. Instead, most panels depict recognizable situations that may imply story-like scenarios: a footman tumbling down with his tray on the floor in the "Ladies' Withdrawing Room"; toasting and heavy drinking in the "Gentlemen's Withdrawing Room"; servants playing cards in the Laundry; a crippled man in a Study pulls the bell, while trying with his crutch to stop a dog from biting a cat; a footman hugging a coy maidservant in the Kitchen; and so on. The space of the house, thereby, allows the humorous portrayal of a cross-section of society and many evolving simultaneous situations in the same space.

Beyond such descriptive uses of the image sequence, humorous pictorial alphabets, calendars, and dictionaries, ${ }^{21}$ or mock instructions ${ }^{22}$ can also be given in the strip design in this historical context. Thus, the panels may be related to each other by some shared topic or conceptual frame rather than a narrative. We must note, furthermore, that the distinction between a strip sequence and a single image is not always that clear cut in this body of work. One case in point is the long panorama image that has the potential to be read both as a scene where many situations occur simultaneously, and as a series of situations, and thus as a kind of strip without the panel frames. The amount of visual detail in a typical panoramic broadsheet print, depicting for instance a procession, a ball, a dinner party, or a pub scene, such as Gillray's "Union Club" (1801) or "The Grand Coronation Procession of Napoleon the 1st" (1804), gives a scene of rich and lively simultaneity. At the same time, certain features of the image content, such as a road or the portrayal of movement into a particular direction, may imply that the image consists of various distinct units of attention set in a certain order. 
The sense of an image sequence can also be heightened by the material form of the publication, such as when the work requires the reader to unfold or uncoil the image in order to see it in full. ${ }^{23}$ For instance, Robert Cruikshank's "Going to a Fight" (1819), ${ }^{24}$ which depicts in forty-two scenes with Londoners going to see a boxing match, and the subsequent match, requires the viewer to uncoil eight broad picture sheets from a spool and boxwood drum. The numbered scenes of this panorama strip, showing various styles of clothing, journeying, and boxing enthusiasm, and that relate some story-like situations such as pickpocketing, are meant to be viewed from right to left. The order of viewing is further confirmed by the direction of movement in many of the scenes. In comparison, the various 'three-dimensional' features in Glasgow Looking Glass/Northern Looking Glass investigate the issue of perspective rather than strengthen the sense of sequence. Perhaps the most impressive of these innovations is the multi-perspective cartoon entitled "All My-Eye", from the cover of the fourth issue of Glasgow Looking Glass (23 July 1825), which shows how the same eyes, forehead, and hair may serve for two different people and facial expressions. Here, a piece of paper, on which is depicted the features of the lower part of a face, is superimposed on another face underneath. By flipping the paper, which is pasted to the page by one margin, the reader can thus perceive two faces in one image. ${ }^{25}$

Many of the larger images in Every Body's Album, such as "The Century of Invention. Anno domini 2000"26 (1 February 1834) that depicts a scene with futuristic means of transportation, including flying machines, moveable houses, cars, and buses, function similarly to the richly detailed panoramic broadsheets. In addition to the global look on a transportation utopia that this scene suggests, the many speech balloons and dialogue situations within the image imply that the composition consists of various simultaneous situations and distinct units of attention.

By contrast, in Glasgow Looking Glass/Northern Looking Glass, panoramic scenes are relatively uncommon. However, what becomes important in terms of simultaneity in these caricature magazines is the question of the page as a unit of attention and reading. In some large full-page or half-page compositional units in Northern Looking Glass and The Looking Glass, the page layout suggests effects of simultaneity, multiple possible perspectives and the global look across the whole page. In these cases, the significance of the page as a design unit involves both the conception of the page as a whole and its division into distinct cartoons, sequences, and zones of composition. One of the most impressive compositions in this regard is "St. Michael of London" from The Looking Glass (No. 8, 1830), which depicts Bishop Charles Blomfield, poised on a cushion in front of a dark cloud, reminiscent of the hovering Christ in church paintings, suspended above and in front 


\section{0}

of the little scenes given in three panels. What is particularly remarkable here is that the bishop's caricature serves as a link between the surrounding contrasted panels and strips (Figure 10.6). The Bishop, exclaiming "Profane Wretch!!!", spears a leg of mutton with a lance on a poor man's dish in the panel below, thus shattering the dish. On the left, the Bishop's cloud extends to a panel that shows a group of soldiers carrying large dishes from a kitchen, while a military band plays "Go to the Devil and shake yourself", and The Duke of Wellington greets them from the balcony of his Apsley House, with the new statue of Achilles (as Wellington) in the background. To the right of the bishop, in contrast, we see groups of poor people driven by canons "back to the smoke on a Sunday", and two individuals who hold their ground, claiming that "Ve vont go back".

In "St. Michael of London", the juxtaposition of elements is more important and suggestive as a principle of organisation than narrative sequence. At the same time, the composition of this page experiments

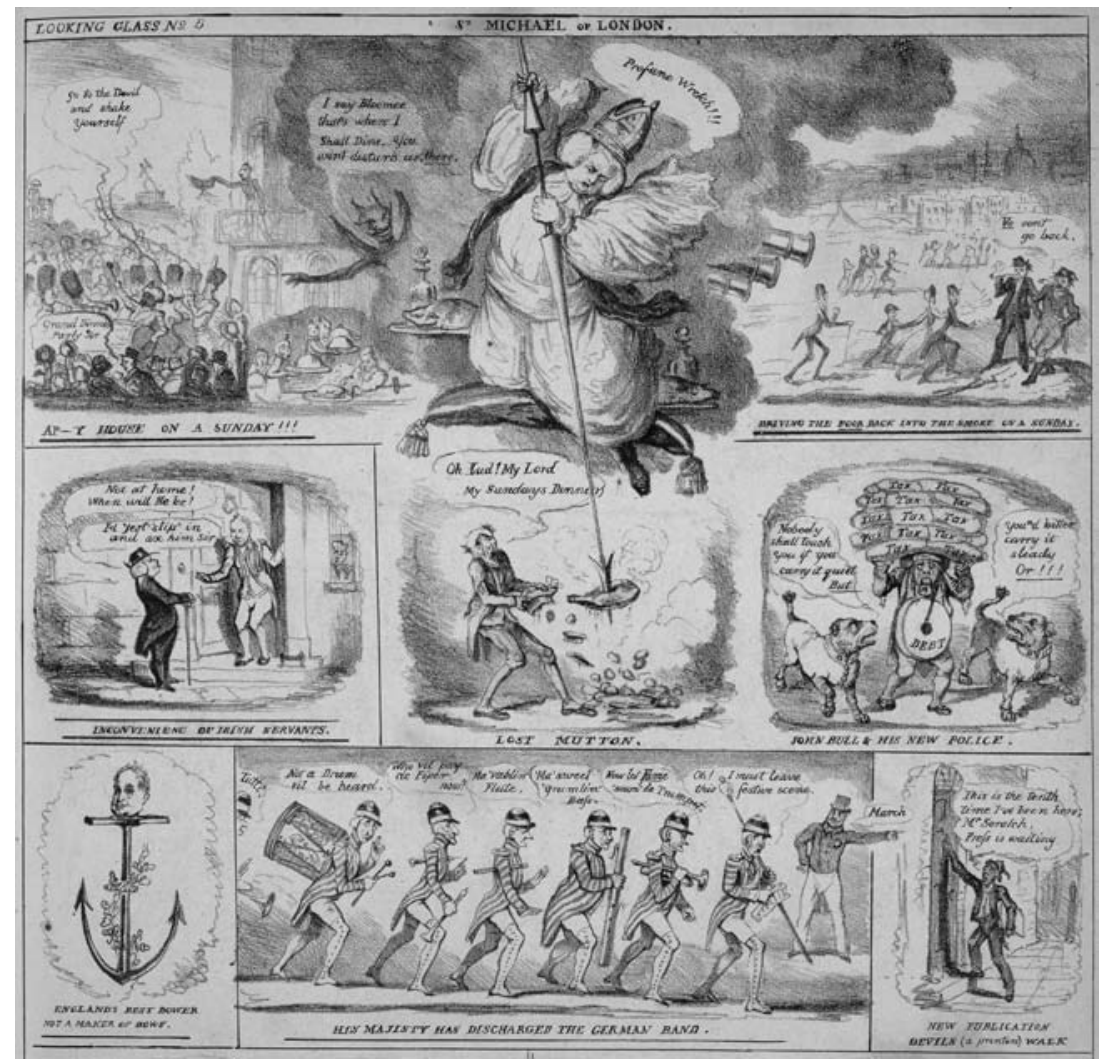

Figure 10.6 Robert Seymour. "St. Michael of London". The Looking Glass, No. 8, 1 August 1830. () The British Library Board, RB.31.c.31. 
with page layout in ways that are familiar in modern comics albums and graphic novels. For instance, there are several repeated patterns and situations across the page of this T-shaped composition that suggest ways of looking beyond the strip sequence. The repeated elements include the topoi of a military band, a scene at a street door, and The Duke of Wellington looking at a crowd from a balcony (Apsley House and the Buckingham Palace). Their patterns suggest multiple ways for looking at the page beyond the single cartoon or strip and extend the influence of the "St Michel of London" and the bishop's figure over the surrounding frames, dominant in the upper part of the page, to other parts of the page.

Also, the lower part of the page includes a breach with the convention of the panel frame. This occurs in a cartoon entitled "His Majisty (sic!) has discharged the German band", where a German band is seen marching in a file from left to right. The new King William IV, who had dismissed his predecessor George IV's band, stands in the background with his arm extended beyond the panel frame to the next image, commanding the musicians imperiously to "March". The foremost marcher to the right, approaching the frame of the panel, states that he must leave the scene: "Oh, I must leave this festive scene". The subsequent cartoon, a parodical reference to Robert Southey's new edition of his and Samuel Taylor Coleridge's poem "The Devil's Walk", is unrelated in subject matter with the previous panel. However, the King's arm has the function, from the reader's viewpoint, of a kind of viewing and reading instruction that connects the two cartoons and gives a sense of direction on the page, which is affirmed by the band members' movement. The panel frame, then, appears here as a convention that can be broken. The crossed frame not only affirms the linear order of reading but also ironically gestures towards the reader about the artificial nature of this convention.

The page of "St. Michael of London" is one of the many innovations in this body of work that suggests that the broadsheet caricature magazines of 1825-1835 offered opportunities for creating new types of relation between the units on the page and the page layout, thus developing the earlier graphic inventions in the broadsheet format. This means not just a stronger reliance on the strip format and the image sequence, but the treatment of the space of the page as one unit of composition and attention. Many front covers of Northern Looking Glass and The Looking Glass work similarly to "St Michel of London" in that they reflect a certain spatial dynamic and hierarchy in the global organisation of the page. Typically, the covers feature a more centralized image, which breaks with the grid-like layout of the unrelated cartoons. For instance, in the eighth issue of The Looking Glass (1 August 1830), the images of William IV and Queen Adelaide's heads in the large central column radiate over the frames of the surrounding cartoons. Below them, in an even more prominent and central design, a terrifying 
black giant in a general's uniform intimidates a small dandy demanding: "How dare you appear without a black coat?". The caption reads: "General Mourning!!" in reference to George IV's death in June 1830. The corners of the black-bordered and diamond-shaped rectangle are superimposed on the surrounding vignettes, with no particular connection with the topic. Sometimes the cover page composition may also be thematically unified, as is the case with the cover of the fifth issue of Glasgow Looking Glass (6 August 1825) with the title "A State of the Weather". Here, all cartoons of the page portray, more or less, the theme of torrid weather and seasonal conditions. In the central upper part of the page, the rays of sun strike downwards, thus connecting a group of five images and suggesting that they have a similar relation to the central image of the flaming sun. Enclosed in this sun is a tiny figure of Apollo, who blows the flames with bellows.

\section{Conclusion}

The caricature magazines that were published in England and Scotland from 1825 to 1835, including especially Glasgow Looking Glass/ Northern Looking-Glass, The Looking Glass or, Caricature Annual, and Every Body's Album and Caricature Magazine, played a significant historical role in the gradual development of modern comics. We can conceive their significance as a kind of waypoint between the broadsheet prints and the comic strip, the comic magazine, and the comic book from several perspectives. The artists who were central creators in these publications, such as William Heath, Charles Jameson Grant, and Robert Seymour, carried on the graphic experimentation of the Golden Period of caricature and the broadsheet print, helping to translate the styles and graphic innovations of the earlier masters, such as James Gillray, into the format of the printed press and the architecture of a magazine page. These experiments included, in particular, the sequential form, the development of the progress story in the multipanel format of the strip design, the narrative conception of panel relations, and the treatment of space of the composition both as a design unit and a unit of attention.

The organisational principles of juxtaposition, sequentiality, and simultaneity, or their combinations, play a significant role in this body of narrative drawings. Heuristically, we can distinguish two basic forms of the sequential form with regard to the implied temporal and causal relations between the images: the episodic sequential form and the narrative continuity sequence, even if their distinction is not always that clear-cut. While the sequential form is often (but not always) closely associated with narrative function, the principle of juxtaposition can equally well serve narrative function, besides doing something else, such as the illumination of an idea, a theme, a metaphor, or contrasting viewpoints, arguments, personalities, and behavior. 
William Heath, Charles Jameson Grant, Robert Seymour, and their colleagues explored the relationship between the strip design, the image sequence, and the space of the composition in the architecture of the magazine page, continuing the art of caricature of the single-plate street broadsheet woodcuts in a new periodical format that used litographed comic images. The new publication format offered new freedoms. Their satirical magazines, or magazine-like broadsheet prints in the case of Grant's Every Body's Album, showcase various examples of a more global conception of the page, and the zone of composition, beyond a single image and a strip. Thus, the page layout and the space of the composition become important at two levels simultaneously: as a design unit and a unit of attention. At the same time, this exploration concerns the relation between the strip design and the sense of narrative sequence, and the distinction that we today may take granted between a cartoon, that is, a single-image drawing, and a sequential narrative comic. The idea of a panel set in relation to surrounding panels, in a sequence or otherwise, is already a convention in this body of work and one that can be consciously broken-even if there are also strong indications that the sequential arrangement of narrative drawings was not that familiar with all readers. All in all, the new effects of sequentiality in these works, often combined with the logic of juxtaposition or simultaneity, met the issue of visual narrativity in a novel way, thus also implying the emergence of new types of the reader's 'diegetic absorption' through an image series.

Finally, it must be emphasised that in my choice of examples, I have focussed on picture stories and their narrative organisation. There are also various non-narrative forms for organising series or groups of images and using the strip design in this body of work. They comprise description and exposition, as in illustrations to a text (poems, stories, anecdotes), pictorial encyclopedia or dictionary, inventory or the cataloguing of items, objects, and so on-based on the principles of accumulation or aggregation, instead of juxtaposition or sequentiality-puns and jokes, and political satire without a sense of a story. We must thus note that the variety of forms of graphic art in this body of work go vastly beyond the narrative sequence and the need to follow a story from one panel to another. While most comics since then have been narratives, comics have never been just narratives.

\section{Notes}

1 Such as the title page, cover artwork, copyright pages, foreword, epigraphs, epilogue, or publishers' blurbs.

2 Lefèvre specifies that a publication format is not only defined by its material aspects (size, paper quality, etc.), but also by its temporal (daily, weekly, monthly, one shot) and editorial parameters (length of an episode, regulations regarding content or the public) $(2013,267)$, as well as by thematic constraints and an aesthetic system $(2009,227 ; 2010,88,91)$. 
3 See also Fox $(1988,76)$.

4 Including albums, such as Ally Sloper: A Moral Lesson (1873), and comic magazines. Often referred to as the first comic magazine, it is debatable, however, to what extent the weekly Ally Sloper's Half-Holiday actually was a comic magazine in its early years. The emphasis on single-image cartoons and written articles made the weekly look much like any other satirical periodical of this time. That said, however, we can also follow the development of the continuing character and the sequential story in this publication. The longest comics in the first year of its publication in 1884 include around ten panels, while the story entitled "Toddleboy goes to see the university crews practice" from 27 March 1886 already has twenty-one panels. On the history of early British publication venues for comics, with a specific emphasis on children's comics, see also Gifford (2004, 362-371). See Beringer on the development of the multipanel picture story in American illustrated magazines from the 1850s on, including Yankee Notions (1852-1875), Nick Nax (1856-1875), and the Comic Monthly (1859-1891), and how these developments were defined "in large part by a taste for experimenting with new and different ways of depicting narrative experience" $(2015,457)$.

5 Subtitled "One hundred laughs for one halfpenny", the first issue was published on 17 May 1890.

6 The Barnhart Dictionary of Etymology (1988) claims that the meaning of comics as a "comic strip" is first recorded as late as 1910 in H.G. Wells' comic novel The History of Mr. Polly where there is a reference to cheap boys' comics of today.

7 My distinction between the strip design and the sequential form follows Kunzle's corresponding definitions (quoted here) as well as Groensteen's distinction between a string (or a series) of panels and a sequence. For Groensteen, a sequence is different from the mere juxtaposition of disparate images or a series that is based on visual, iconic, or semantic correspondences between the images, in that in a sequence the "syntagmatic linkage" in the succession of images is "determined by a narrative project" $(2014,176)$.

8 As in James Gillray's drawing "Nature display'd, shewing the effect of the change of the seasons on the ladies garden" (1797), where the four headless female figures need to be viewed from four different angles.

9 Compare with Beringer's emphasis on the diversity of ways for organising the narrative flow in comics published in the 1850s American humour magazines comics. In this context, Beringer argues, it was more likely that the panel transitions were organised in ways that do not follow a tight sequence of actions, but, for instance, through movement between various scenes, or the change of perspective $(2015,457)$.

10 See, for instance, Smolderen (2014a, 137-148) and Lefèvre (2006).

11 The first issue of Glasgow Looking Glass appeared on 11 June 1825. It was renamed Northern Looking Glass in the issue VI (18 August 1825) and Northern Looking Glass, or Litho's Album in the next issue (3 September 1825). In the early stages of his career, Charles Jameson Grant collaborated with William Heath's brother, Henry, and also produced a small number of prints for William Heath and Robert Seymour's publisher, Thomas McLean (see Pound 1998).

12 Beyond the satirical and comic images, the issues of 1-4 of Glasgow Looking Glass feature extracts from Horace Smith's and Thomas Campbell's poems. The last page of the second issue of Northern Looking Glass (VII) comprises quotes from newspapers and periodicals. This becomes a permanent feature in the subsequent issues. The first issue of the short-lived "new series" of 
Northern Looking Glass (May 1826) includes an editorial introduction and texts relating to some of the images.

13 Kunzle refers to the antithetical method of narration in picture stories before the modern comic strip: "To narrate is, first of all, to polarize a sequence of events into Before and After, Then and Now, Cause and Result-and Crime and Punishment" $(1973,3)$. Thus, we may add, the logic of this method is either temporal or causal.

14 The subtitle explains that this takes place in France and, further, that "The following is the manner in which the King of France passes his time at St. Cloud" (Château de Saint-Cloud).

15 The contrast is further affirmed by the motto taken from Hamlet: "Look here upon this picture and on this, The counterfeit presentment of two brothers".

16 "Hope" can be further contrasted with another Gillray caricature, "Despair", that was published at the same time and that shows the opposition leader Richard Bateman Robson speaking in the House of Commons and stating that "We're all ruinated, Sir!".

17 The title is reminiscent of Heath's earlier illustrated narrative poem, The Life of a Soldier; A Narrative and Descriptive Poem (1823), but the images in the magazine are much simpler and more caricature-like than in the engravings of the illustrated book.

18 Another instance of seemingly wrong order in Northern Looking Glass (No. X) involves a two-panel story entitled "Cambridge Sporting Intelligence”. In this incident, a Dr Syntax-like character, who is carried on another person's shoulders in a piggyback race, first falls down and is then again shown racing in the piggyback position. See also Witek, who points out how the use of panel numbering and directional arrows in the early history of the medium suggests how "comicness" can be conceptualised as "a historically contingent and evolving set of reading protocols that are applied to texts" $(2009,149)$.

19 Another version of this are picture stories where different phases in a character's or a group's development are shown in a long strip-like space without separating frames. For instance, in George Woodward and F. G. Byron's "The Clerical Exercise" (1791).

20 It has been claimed that the miniature version of "Harlot's Progress" that was published in June 1828 in the English weekly Bell's Life in London, and Sporting Chronicle (1822-1886) was the first newspaper strip (Kunzle 1973, 162; Gravett 1998). The claim can be contested in light of the strips featured in Glasgow/Northern Looking-Glass in 1825-1826. That said, Bell's Life had an important role in the early history of comics by running a weekly feature called "The Gallery of Comicalities", including a series of caricatures and illustrated jokes, beginning in September 1827. These caricatures were then collected in the broadsheet newspaper The Gallery of Comicalities, and published every 2 years between 1832 and 1841 .

21 Such as Richard Newton's “Clerical Alphabet" (1795) or "The Pictorial Dictionary" in Northern Looking Glass (No. XII).

22 Such as "Six of the most approved methods of appearing ridiculous on the ice" or "symptoms of jolting" (in stage coaches) in George Woodward's parodic travel book Eccentric Excursions (1796).

23 For instance, the folded political cartoons included in The Scourge and George Cruikshank's The Comic Almanack (1844-1853). The readers' activation in this way may, however, focus on perspectival effects rather than the impression of sequentiality. 
24 Subtitled "Illustrating the Sporting World in all its variety of Style and Costume along the Road from Hyde Park Corner to Moulsey Hurst", accompanied by Pierce Egan's written description, and sized $14 \mathrm{ft}$ by 2.4 inches, this work is based on a true sporting event that took place in April 1817.

25 In another issue of Northern Looking Glass (No. VII, 3 September 1825), a cavalry officer's hat extends beyond the panel in a separate piece of folded paper, similarly pasted on the page.

26 Subtitled "Or the March of Aerostation, Steam, Rail Roads, Moveable Houses, \& Perpetual Motion”. 


\section{Afterword}

It is in the nature of narratology to seek what is most universal, conventional, and general about narratives, and attempt to describe and analyse these features as effectively as possible. Yet, the relationship between what is general and what is unique, variable, or contra-standard in storytelling has also been one of the defining tensions throughout the history of this field. Thus, the impetus towards the generalisable has been regularly counterbalanced by paying attention to the ways in which individual artists and works of art explore, question, and modify common practices and invent new forms of storytelling. When narratology meets the unexpected, and the singular, the theory has to be adjusted-if not right away, then in time. This process of adjusting, then, has become an important outcome of the research.

In today's narratology, the question about the universal and the unique in narratives also extends to the relation between narratives and their media. As narratology has reached outside the traditional object domain of text-based literary story, it has increasingly started to pay attention to the ways in which narrative transmission is media-related and how the qualities of the medium affect the way in which stories can be told. In this book, I have attempted to take the medium-specific argument as far as possible, but always keeping in mind the shared qualities of narratives across many different systems of communication and expression, especially with literature and cinema. The principle that I have followed is that for narratology to have general relevance in the field of Comics Studies, it needs to adopt a self-critical and flexible attitude towards theoretical propositions in the light of actual poetics and artistic choices in this form of art. And, this is how I see the relevance of the examples that I have discussed in this study: they illustrate the main narrative conventions in the modern Western comics, reflecting diversity in historical context, markets, language, genre, and publication format, while many of them also illustrate how individual works can successfully modify the existing narrative traditions and forms to establish their rightful place in the canons of literary expression and visual art.

I hope to have demonstrated that I do not conceive narratology as a solution to all interpretive challenges that may be faced in studying 
comics. Narratological concepts and approaches are a valuable source of insight in research, where they can stretch our awareness of the narrative qualities of this art and its historically contingent forms, thus preparing the way for a more holistic interpretation. A more comprehensive historical study of comics, for instance, must engage with many other dimensions of comics beyond their narrative form, conventions, and strategies, including the question of genre (generic relations, implicatures, and expectations), intertextual and intermedial relations, the context of the making and reading of comics, artistic and stylistic movements, authorial intention and readerly expectations, and relevant aspects of the culture and materiality of comics, such as publication formats and distribution, fandom, criticism, and marketing. The range of potentially relevant contexts for studying comics in the humanities and social sciences is, in principle then, infinite.

One last matter that I wish to draw to the reader's attention is the appreciation of comics as a form of reading. It has sometimes been claimed that narratology seeks to unravel the mysteries of storytelling, i.e. expose the bare essence of the narrative form, and that for this reason its analytical procedures could result in killing the joy of reading, viewing, or listening to narratives. Personally, I do not believe that narratology has any such a 'demystifying' objective or that narratological analysis needs to be detrimental to the pleasures of reading. On the contrary, my experience in teaching narratology and doing research in this field, and having discussed the point of narrative analysis with cartoonists and students, is that narrative theory can complement personal as well as expressive and artistic responses to the art of comics. Narrative theory may even have, when used judiciously, the potential to intensify such pleasures and responses. On a more personal note, this book has also been inspired by my own personal experiences of reading comics, marvelling at them, and enjoying them throughout my life. Having first learned to read by way of comic books, I have posed questions to myself such as: 'Why are comics so interesting and appealing as narratives?' and 'What is particularly effective about telling stories in this form of art and literature?' The process of writing The Narratology of Comic Art has been a meaningful way to explore such questions. 


\section{Bibliography}

Abbott, Lawrence L. 1986. "Comic Art: Characteristics of a Narrative Medium.” Journal of Popular Culture 19: 155-76.

Abbott, Porter H. 2005. "Closure." In Routledge Encyclopedia of Narrative Theory, 65-66. Eds. David Herman, Manfred Jahn and Marie-Laure Ryan. London and New York: Routledge.

- 2008. The Cambridge Introduction to Narrative. 2nd ed. Cambridge: Cambridge University Press.

Altman, Rick. 2008. A Theory of Narrative. New York: Columbia University Press.

Ault, Donald. 2004. "Preludium: Crumb, Barks, and Noomin: Re-considering the Aesthetics of Underground Comics." ImageTexT 29. http://www.engl...s/ v1_2/intro.shtml.

Badman, Derik A. 2010. "Talking, Thinking, and Seeing in Pictures: Narration, Focalization, and Ocularization in Comics Narratives." International Journal of Comic Art 12.2/3: 91-111.

Baetens, Jan. 1989. Hergé écrivain. Bruxelles: Labor.

- 1998. Formes et politique de la bande dessinée. Leuven: Peeters Vrin. 2001. "Revealing Traces: A New Theory of Graphic Enunciation." In

The Language of Comics: Word and Image, 145-55. Eds. Robin Varnum and Christina T. Gibbons. Jackson: University Press of Mississippi.

—. 2004. "La main parlante." Image \& Narrative V.1. http://www.imageand narrative.be/inarchive/performance/baetens_main.htm.

- 2008. "Graphic Novels: Literature without Text?" English Language Notes 46.2: 77-88.

—. 2013. "Uncaging and Reframing Martin Vaugh-James's the Cage." In Drawing from Life: Memory and Subjectivity in Comic Art, 67-85. Ed. Jane Tolmie. Jackson: University Press of Mississippi.

Baetens, Jan and Hugo Frey. 2015. The Graphic Novel. An Introduction. Cambridge and New York: Cambridge University Press.

Baetens, Jan and Pascal Lefèvre. 1993. Pour une lecture moderne de la bande dessinée. Bruxelles: Centre belge de la bande dessinée.

Bal, Mieke. 1981. "Notes on Narrative Embedding." Poetics Today 2.2: 41-59.

—. 1990. "The Point of Narratology." Poetics Today 11.4: 727-53. 1991. On Story-Telling. Essays in Narratology. Ed. David Jobling. Sonoma: Polebridge Press.

1997. Narratology: Introduction to the Theory of Narrative. 2 nd ed.

Toronto: University of Toronto Press. 


\section{Bibliography}

Barker, Martin. 1989. Comics: Ideology, Power, and the Critics. Manchester: Manchester University Press.

Bateman, John A., Francisco O. D. Veloso, Janina Wildfeuer, Felix HiuLaam Cheung and Nancy Songdan Guo. 2016. "An Open Multilevel Classification Scheme for the Visual Layout of Comics and Graphic Novels: Motivation and Design.” Digital Scholarship in the Humanities 1-35. doi:10.1093/llc/ fqw024.

Bavelas, Janet B. and Nicole Chovil. 2006. "Hand Gestures and Facial Displays as Part of Language Use in Face-to-Face Dialogue.” In The Sage Handbook of Nonverbal Communication, 97-115. Eds. Valerie Manusov and Miles

L. Patterson. Thousand Oaks, CA, London and New Delhi: Sage Publications.

Beaty, Bart. 1999. "The Search for Comics Exceptionalism." Comics Journal 211: 67-72.

- 2012. Comics versus Art. Toronto: Toronto University Press.

Benveniste, Émile. 1970. “L'appareil formel de l'énonciation.” Langages 5.17: 12-18.

Beringer, Alex. 2015. "Transatlantic Picture Stories: Experiments in the Antebellum American Comic Strip." American Literature 87.3: 455-88.

Blackwell, Mark. 2012. "General Introduction." In British It-Narratives, 1750-1830. Volume I (Money), vii-xxviii. Ed. Liz Bellamy. London: Pickering \& Chatto.

Boillat, Alain (ed.). 2010. Les Cases à l'écran. Bande dessinée et cinéma en dialogue. Genève: Éditions Georg/L'Equinoxe.

Bongco, Mila. 2000. Reading Comics. Language, Culture, and the Concept of the Superhero in Comic Books. New York: Garland.

Booth, Wayne C. 1983. The Rhetoric of Fiction. 2nd ed. Chicago and London: University of Chicago Press.

Bordwell, David. 1985. Narration in the Fiction Film. Madison: University of Wisconsin Press.

- 2008. Poetics of Cinema. New York: Routledge.

Bordwell, David and Kristin Thompson. 1986. Film Art: An Introduction. 2nd ed. New York: Alfred A. Knopf.

- 2008. Film Art. An Introduction. 8th ed. Boston: McGraw-Hill.

Bortolussi, Marisa and Peter Dixon. 2003. Psychonarratology: Foundations for the Empirical Study of Literary Response. Cambridge: Cambridge University Press.

Bramlett, Frank. 2012. "Linguistic Codes and Character Identity in Afro Samurai." In Linguistics and the Study of Comics, 183-209. Ed. Frank Bramlett. London: Palgrave Macmillan.

Branigan, Edward. 1984. Point of View in the Cinema: A Theory of Narration and Subjectivity in Classical Film. Berlin: Mouton.

-1992. Narrative Comprehension and Film. London: Routledge.

Bredehoft, Thomas A. 2006. "Comics Architecture, Multidimensionality, and Time: Chris Ware's Jimmy Corrigan: The Smartest Kid on Earth.” Modern Fiction Studies 52.4: 869-90.

—. 2011. "Style, Voice, and Authorship in Harvey Pekar's (Auto) (Bio) Graphical Comics." College Literature 38.3: 97-110.

Bremond, Claude. 1968. "Pour un gestuaire des bandes dessinées." Langages 3.10: 94-100. 
Bridgeman, Teresa. 2004. "Keeping an Eye on Things: Attention, Tracking and Coherence-Building.” Belphégor: Littérature Populaire et Culture Médiatique 4.1. http://etc.dal.ca/belphegor/vol4_no1/articles/04_01_Bridge_keep_ en_cont.html.

Brooke-Rose, Christine. 1981. A Rhetoric of the Unreal. Studies in the Narrative and Structure, Especially of the Fantastic. Cambridge: Cambridge University Press.

Bukatman, Scott. 2006. "Comics and the Critique of Chronophotography, or 'He Never Knew When It Was Coming!'” Animation 1: 83-103.

- 2014. "Sculpture, Stasis, the Comics, and Hellboy." Critical Inquiry 40.3: 104-17.

Butte, George. 2008. "Suture and the Narration of Subjectivity in Film." Poetics Today 29.2: 277-308.

Carrier, David. 2000. The Aesthetics of Comics. University Park: The Pennsylvania State University Press.

Carroll, Noël. 2008. The Philosophy of Motion Pictures. Malden, MA and Oxford: Blackwell Publishing.

Chapman, Raymond. 1984. The Treatment of Sounds in Language and Literature. Oxford: Blackwell.

Chatman, Seymour. 1978. Story and Discourse: Narrative Structure in Fiction and Film. Ithaca, NY: Cornell University Press.

-1990. Coming to Terms: The Rhetoric of Narrative in Fiction and Film. Ithaca, NY: Cornell University Press.

Chavanne, Renaud. 2010. Composition de la Bande dessinée. Montrouge, France: Editions PLG/Collection « Mémoire Vive ».

Christiansen, Hans-Christian. 2000. "Comics and Film: A Narrative Perspective." In Comics \& Culture: Analytical and Theoretical Approaches to Comics, 107-22. Eds. Anne Magnussen and Hans-Christian Christiansen. Copenhagen: University of Copenhagen/Museum Tusculanum Press.

Chute, Hillary. 2010. Graphic Women: Life Narrative and Contemporary Comics. New York: Columbia University Press.

Chute, Hillary and Marianne DeKoven. 2006. "Introduction: Graphic Narrative." Modern Fiction Studies 52.4: 767-82.

Cohan, Steven and Linda M. Shires. 1988. Telling Stories: A Theoretical Analysis of Narrative Fiction. New York: Routledge.

Cohn, Dorrit. 1978. Transparent Minds: Narrative Modes for Presenting Consciousness in Fiction. Princeton, NJ: Princeton University Press.

- 1999. The Distinction of Fiction. Baltimore, MD: The Johns Hopkins University Press.

Cohn, Jesse. 2007. “Translator's Comments on Benoît Peeters, 'Four Conceptions of the Page.'” ImageText 3.3. http://www.english.ufl.edu/imagetext/ archives/v33/cohn/.

- 2009. "Mise-en-Page: A Vocabulary for Page Layouts." In Teaching the Graphic Novel, 44-57. Ed. Stephen E. Tabachnick. New York: The Modern Language Association of America.

Cohn, Neil. 2007. "A Visual Lexicon." The Public Journal of Semiotics 1.1: 35-56.

- 2010. "The Limits of Time and Transitions: Challenges to Theories of Sequential Image Comprehension.” Studies in Comics 1.1: 127-47. 


\section{Bibliography}

2013. "Navigating Comics: An Empirical and Theoretical Approach to Strategies of Reading Comic Page Layouts." Frontiers in Psychology 4.186: $1-15$.

- 2014a. "The Architecture of Visual Narrative Comprehension: The Interaction of Narrative Structure and Page Layout in Understanding Comics." Frontiers in Psychology 5.680: 1-9.

- 2014b. "Building a Better 'Comic Theory': Shortcomings of Theoretical Research on Comics and How to Overcome Them." Studies in Comics 5.1: 57-75.

Cortsen, Rikke P. 2012. Comics as Assemblage: How Spatio-temporality in Comics Is Constructed. PhD dissertation. University of Copenhagen.

Covey, Suzanne. 2006. "Beyond the Balloon: Sound Effects and Background Text in Lynn Johnston's For Better or For Worse." ImageTexT: Interdisciplinary Comics Studies 2.2. http://www.engl.../covey/index.shtml.

Cutting, James E. 2014. "Event Segmentation and Seven Types of Narrative Discontinuity in Popular Movies.” Acta Psychologica 149: 69-77.

Delany, Samuel R. 1999. "Politics of Paraliterary Criticism." In Shorter Views. Queer Thoughts \& The Politics of the Paraliterary, 218-70. Ed. Samuel R. Delany. Hanover and London: Wesleyan University Press.

Deleyto, Celestino. 1996. "Focalisation in Film Narrative." In Narratology: An Introduction, 217-33. Eds. Susana Onega and José Angel Garcia Landa. London: Longman.

Donaldson-Evans, Mary. 2009. Madame Bovary at the Movies: Adaptation, Ideology, Context. Amsterdam and New York: Éditions Rodopi.

Duncan, Randy and Matthew J. Smith. 2009. The Power of Comics. History, Form, and Culture. New York: Bloomsbury.

Dürrenmatt, Jacques. 2013. Bande dessinée et littérature. Paris: Classiques Garnier.

Eisner, Will. 1985. Comics \& Sequential Art. Tamarac, FL: Poorhouse Press.

- 1996. Graphic Storytelling and Visual Narrative. Tamarac, FL: Poorhouse Press.

Feiffer, Jules. 1965. Great Comic Book Heroes. London: Dial Press.

Fein, Ofer and Asa Kasher. 1996. "How to Do Things with Words and Gestures in Comics." Journal of Pragmatics 26: 793-808.

Fischer, Craig and Charles Hatfield. 2011. "Teeth, Sticks, and Bricks: Calligraphy, Graphic Focalization, and Narrative Braiding in Eddie Campbell's Alec." SubStance 40.1: 70-93.

Fishelov, David. 1990. "Types of Characters, Characteristics of Types.” Style 24: 422-39.

Flaubert, Gustave. 1946. Madame Bovary. Provincial Manners. Trans. Eleanor Marx Aveling. New York: The Modern Library.

- 1993. Madame Bovary. Paris: Booking International.

Fludernik, Monika. 1996. Towards a 'Natural' Narratology. London and New York: Routledge.

- 2001. "New Wine in Old Bottles? Voice, Focalization, and New Writing.” New Literary History 32: 619-38.

- 2003. "Scene Shift, Metalepsis, and the Metaleptic Mode." Style 37.4: 382-400. 
2009. An Introduction to Narratology. Trans. Patricia Häusler Greenfield and Monika Fludernik. London: Routledge.

Forceville, Charles. 2002. "The Conspiracy in The Comfort of Strangers: Narration in the Novel and the Film." Language and Literature 11.2:119-35. - 2005. "Visual Representations of the Idealized Cognitive Model of Anger in the Asterix Album La Zizanie." Journal of Pragmatics 37: 69-88.

- 2011. "Pictorial Runes in Tintin and the Picaros." Journal of Pragmatics 43: 875-90.

- 2013. "Creative Visual Variation in Comics Balloons." In Creativity and the Agile Mind: A Multi-disciplinary Study of a Multi-faceted Phenomenon, 251-73. Eds. Tony Veale, Kurt Feyaerts and Charles Forceville. Berlin: Mouton de Gruyter.

Forceville, Charles, Elisabeth E. Refaie and Gert Meesters. 2014. "Stylistics and Comics." In The Routledge Handbook of Stylistics, 485-99. Ed. Michael Burke. London: Routledge.

Forceville, Charles, Tony Veale and Kurt Feyaerts. 2010. "Balloonics: The Visuals of Balloons in Comics." In The Rise and Reason of Comics and Graphic Literature. Critical Essays on the Form, 56-73. Eds. Joyce Goggin and Dan Hassler-Forest. Jefferson, NC and London: McFarland Press.

Forster, Edward Morgan. 1927/1953. Aspects of the Novel. London: Edward Arnold \& Co.

Fowler, Roger. 1977. Linguistics and the Novel. London: Methuen.

Fox, Celina. 1988. Graphic Journalism in England during the 1830s and 1840 s. New York and London: Garland.

Fresnault-Deruelle, Pierre, 1976. "Du linéaire au tabulaire.” Communications 24: 7-23.

- 1977. Récits et discours par la bande. Essais sur les comics. Paris: Hachette.

Gardner, Jared. 2011. "Storylines.” SubStance 40.1: 53-69.

- 2012. Projections. Comics and the History of Twenty-First-Century Storytelling. Stanford, CA: Stanford University Press.

—. 2013. "A History of the Narrative Comic Strip." In From Comic Strips to Graphic Novels: Contributions to the Theory and History of Graphic Narrative, 241-53. Eds. Daniel Stein and Jan-Noël Thon. Berlin and New York: Walter de Gruyter.

Gardner, Jared and David Herman. 2011. "Graphic Narratives and Narrative Theory. Introduction.” Substance 40.1: 3-13.

Gaudreault, André. 1987. "Narration and Monstration in the Cinema." Journal of Film and Video 39.2: 29-36.

- 1989. Du littéraire au filmique. Système du récit. Paris: Méridiens Klincksieck.

- 2009. From Plato to Lumière: Narration and Monstration in Literature and Cinema. Trans. Timothy Barnard. Toronto: University of Toronto Press.

Gaudreault, André and François Jost. 1990. Le Récit Cinematographique. Paris: Éditions Nathan, Collection Nathan-Université.

1999. "Enunciation and Narration." In A Companion to Film Theory, 45-63. Eds. Toby Miller and Robert Stam. Malden, MA: Blackwell Publishing Ltd. 


\section{Bibliography}

Gaut, Berys. 2004. "The Philosophy of the Movies: Cinematic Narration." In The Blackwell Guide to Aesthetics, 230-53. Ed. Peter Kivy. Malden, MA: Blackwell Publishing.

Genette, Gérard. 1980. Narrative Discourse: An Essay in Method. Trans. Jane E. Levin. Ithaca, NY: Cornell University Press.

1988. Narrative Discourse Revisited. Trans. Jane E. Lewin. Ithaca, NY: Cornell University Press.

Gibson, James J. 1979. The Ecological Approach to Visual Perception. Boston: Houghton Mifflin.

Gifford, Denis. 1976. Victorian Comics. London: George Allen \& Unwin Ltd.

- 2004. "Comics, Dime Novels, Pulps and Penny Dreadfuls." In International Companion Encyclopedia of Children's Literature, 362-84. 2nd ed. Ed. Peter Hunt. London and New York: Routledge.

Gitelman, Lisa. 2006. Always Already New. Media, History, and the Data of Culture. London: The MIT Press.

Gombrich, Ernst M. 1960. Art and Illusion. A Study in the Psychology of Pictorial Representation. London: Phaidon Press.

Gravett, Paul. 1998. "The Cartoonist's Progress: The Inventors of Comics in Great Britain.” In Forging a New Medium: The Comic Strip in the Nineteenth Century, 81-103. Eds. Pascal Lefèvre and Charles Dierick. Bruxelles: VUB University Press.

- 2013. Comics Art. London: Tate Publishing.

Groensteen, Thierry. 1988/2014. "Narration as Supplement: An Archaeology of the Infra-narrative Foundations of Comics." In The French Comics Theory Reader, 163-81. Eds. Ann Miller and Bart Beaty. Leuven: Leuven University Press.

_. 1997. "Histoire de la bande dessinée muette." Première partie. Neuvième Art 2: 60-75.

. 1998. "Histoire de la bande dessinée muette." Deuxième partie. Neuvième Art 3: 92-105.

1999. Système de la bande dessinée. Paris: PUF.

. 2007. The System of Comics. Trans. Bart Beaty and Nick Nguyen. Jackson: University Press of Mississippi.

—. 2011. Bande dessinée et narration (Système de la bande dessinée 2). Paris: PUF.

- 2013. Comics and Narration. Trans. Ann Miller. Jackson: University Press of Mississippi.

Guaïtella, Isabelle. 2003. "Au rythme des images: multimodalité et multilinéarité de la bande dessinée." Semiotica 146.1/4: 519-26.

Gunning, Tom. 2009. "Preface." In From Plato to Lumière: Narration and Monstration in Literature and Cinema, xvii-xxv. Ed. André Gaudreault. Toronto: University of Toronto Press.

—. 2014. "The Art of Succession: Reading, Writing, and Watching Comics." Critical Inquiry 40.3: 36-51.

Hague, Ian. 2014. Comics and the Senses. New York and London: Routledge.

Harvey, Robert C. 1994. The Art of the Funnies: An Aesthetic History. Jackson: University Press of Mississippi.

- 1996. The Art of the Comic Book: An Aesthetic History. Jackson: University Press of Mississippi. 
2005. "Describing and Discarding 'Comics' as an Impotent Act of Philosophical Right.” In Comics as Philosophy, 14-26. Ed. Jeff McLaughlin. Jackson: University Press of Mississippi.

Hatfield, Charles. 2005. Alternative Comics: An Emerging Literature. Jackson: University of Mississippi Press.

- 2009. "Defining Comics in the Classroom; or, the Pros and Cons of Unfixability." In Teaching the Graphic Novel, 19-27. Ed. Stephen E. Tabachnick. New York: The Modern Language Association of America.

- 2010. "Indiscipline, or, the Condition of Comics Studies." Transatlantica 1: 2-15. http://transatlantica.revues.org/4933.

Hearn, Lafcadio. 1904. Kwaidan: Stories and Studies of Strange Things. Boston, MA: Houghton, Mifflin and Co.

Herman, David. 1999. “Introduction.” In Narratologies: New Perspectives on Narrative Analysis, 1-30. Ed. David Herman. Columbus: Ohio State Press.

- 2002. Story Logic: Problems and Possibilities of Narrative. Lincoln: University of Nebraska Press.

- 2009a. Basic Elements of Narrative. Chichester: Wiley-Blackwell and John Wiley \& Sons Ltd.

- 2009b. "Beyond Voice and Vision: Cognitive Grammar and Focalisation Theory." In Point of View, Perspective, and Focalisation: Modeling Mediation in Narrative, 119-42. Eds. Peter Hühn, Wolf Schmid and Jörg Schönert. Berlin: Walter de Gruyter.

- 2011. "Storyworld/Umwelt: Nonhuman Experiences in Graphic Narratives." SubStance 40.1: 156-81.

- 2013. Storytelling and the Sciences of Mind. Cambridge, MA and London: MIT Press.

Herman, David and Jared Gardner. 2011. "Graphic Narratives and Narrative Theory: Introduction.” Substance 40.1: 3-13.

Horrocks, Dylan. 2001. "Inventing Comics: Scott McCloud's Definition of Comics.” Comics Journal 234. http://www.hicksville.co.nz/Inventing\%20 Comics.htm.

Horstkotte, Silke and Nancy Pedri. 2011. "Focalization in Graphic Narrative." Narrative 19.3: 330-57.

Jacobs, Dale. 2007. "Beyond Visual Rhetoric: Multimodal Rhetoric and Newspaper Comic Strips.” International Journal of Comic Art 9.1: 502-14.

Jahn, Manfred. 1999. "More Aspects of Focalization. Refinements and Applications." GRAAT: Revue des Groupes de Recherches Anglo-Américaines de L'Université François Rabelais de Tours 21: 85-110.

—. 2003. "A Guide to Narratological Film Analysis." In Poems, Plays, and Prose: A Guide to the Theory of Literary Genres. Cologne: University of Cologne. http://www.uni-koeln.de/ ame02/pppf.htm.

Jannidis, Fotis. 2015. “Character”. In The Living Handbook of Narratology. Eds. Peter Hühn, John Pier, Wolf Schmid and Jörg Schönert. Hamburg: Hamburg University. http://wikis.sub.uni-hamburg.de/lhn/index.php/Character.

Jenkins, Henry. 2006. Convergence Culture: Where Old and New Media Collide. New York: New York University Press.

Jesch, Tatjana and Malte Stein. 2009. "Perspectivization and Focalization: Two Concepts-One Meaning? An Attempt at Conceptual Differentiation." In Point of View, Perspective, and Focalization. Modeling Mediation in 


\section{Bibliography}

Narrative, 59-77. Eds. Peter Hühn, Wolf Schmid and Jörg Schönert. Berlin: Walter de Gruyter.

Jost, François. 1983. "Narration(s): en deçà et au-delà.” Communications 38: 192-212.

-1987. L'Eil-Caméra. Entre film et roman. Lyon: Presses Universitaires de Lyon.

- 2004. "The Look: From Film to Novel: An Essay in Comparative Narratology." In A Companion to Literature and Film, 71-80. Eds. Robert Stam and Alessandra Raengo. Malden, MA: Blackwell.

Kannenberg, Gene Jr. 2001. "The Comics of Chris Ware: Text, Image, and Visual Narrative Strategies." In The Language of Comics: Word and Image, 174-97. Eds. Robin Varnum and Christina T. Gibbons. Jackson: University Press of Mississippi.

Kennedy, John M. 1982. "Metaphor in Pictures.” Perception 11: 589-605.

Kindt, Tom. 2009. "Narratological Expansionism and Its Discontents." In Narratology in the Age of Cross-disciplinary Narrative Research, 35-47. Eds. Sandra Heinen and Roy Sommer. Berlin and New York: Walter de Gruyter.

Kindt, Tom and Hans-Harald Müller. 2003. "Narrative Theory and/or/as Theory of Interpretation." In What Is Narratology? Questions and Answers Regarding the Status of a Theory, 205-19. Eds. Tom Kindt and Hans-Harald Müller. Berlin and New York: Walter de Gruyter.

- 2006. The Implied Author: Concept and Controversy. Berlin and New York: Walter de Gruyter.

Klauk, Tobias and Köppe, Tilmann. 2015. “Telling vs. Showing.” In The Living Handbook of Narratology. Eds. Peter Hühn, John Pier, Wolf Schmid and Jörg Schönert. Hamburg: Hamburg University. http://www.lhn.uni-hamburg.de/ article/telling-vs-showing.

Kolp, Manuel. 1992. Le Langage cinématographique en bande dessinée. Brussels: Éditions de l'université de Bruxelles.

Kozloff, Sarah. 2000. Overhearing Film Dialogue. Berkeley: University of California Press.

Kress, Gunther. 2010. Multimodality: A Social Semiotic Approach to Contemporary Communication. London: Routledge.

Kress, Gunther and Theo van Leeuwen. 1996/2006. Reading Images: The Grammar of Visual Design. 2nd ed. London: Routledge.

Kruger, Jan-Louis. 2012. "Making Meaning in AVT: Eye Tracking and Viewer Construction of Narrative." Perspectives: Studies in Translatology 20.1: 67-86.

Kukkonen, Karin. 2013a. Contemporary Comics Storytelling. Lincoln and London: University of Nebraska Press.

- 2013b. Comics and Graphic Novels. Malden, MA: Wiley Blackwell.

Kunzle, David. 1973. History of the Comic Strip Volume I: The Early Comic Strip. Narrative Strips and Picture Stories in the European Broadsheet from c.1450 to 1825. Berkeley, Los Angeles and London: University of California Press.

- 1990. The History of the Comic Strip Volume II: The Nineteenth Century. Berkeley, Los Angeles and Oxford: University of California Press.

- 2001. "The Voices of Silence: Willette, Steinlen and the Introduction of the Silent Strip in the Chat Noir, with a German Coda." In The Language 
of Comics: Word and Image, 3-18. Eds. Robin Varnum and Christina T. Gibbons. Jackson: University Press of Mississippi.

Labio, Catherine. 2011. "What's in a Name? The Academic Study of Comics and the "Graphic Novel."' Cinema Journal 50.3: 123-26.

Laffay, Albert. 1964. Logique du cinema. Paris: Masson.

Larcenet, Manu. 2011. Interview by Agnès Deyzieux. Le cas des cases. http:// agnesdeyzieux-bd.blogspot.fi/2011/04/larcenet-blast-tome-2.html.

Larsson, Donald. 2000. "Every Picture Tells a Story: Agency and Narration in Film.” Paper given at MLA Annual Conference, Washington, DC. http:// www.english2.mnsu.edu/larsson/agency1.html\#text $\% 201$.

Leech, Geoffrey N. and Michael H. Short. 2007. Style in Fiction: A Linguistic Introduction to English Fictional Prose. 2nd ed. London: Pearson/Longman.

Lefèvre, Pascal. 2005. "The Importance of Being Published." Extended version of original publication in Comics \& Culture, 91-105. Eds. Anne Magnussen and Hans-Christian Christiansen. Copenhagen: Museum Tusculanum at the University of Copenhagen, 2000. https://www.academia.edu/660771/The_ Importance_of_Being_Published_A_Comparative_Study_of_Different_ Comics_Formats.

- 2006. "The Battle over the Balloon. The Conflictual Institutionalization of the Speech Balloon in Various European Cultures." Image \& Narrative VII.1. http://www.imageandnarrative.be/inarchive/painting/pascal_levevre.htm.

- 2007. "Incompatible Visual Ontologies? The Problematic Adaptation of Drawn Images." In Film and Comic Books, 1-12. Eds. Ian Gordon, Mark Jancovich and Matthew P. McAllister. Jackson: University Press of Mississippi.

- 2009. "The Conquest of Space: Evolution of Panel Arrangements and Page Layouts in Early Comics Published in Belgium (1880-1929).” European Comic Art 2.2: 227-52.

- 2010. "Researching Comics on a Global Scale." In Comics Worlds and the World of Comics: Towards Scholarship on a Global Scale, 89-95. Ed. Jaqueline Berndt. Kyoto: International Manga Research Center, Kyoto Seika University

—. 2011. "Some Medium-Specific Qualities of Graphic Sequences.” SubStance 40.1: 14-33.

- 2013. "Narration in the Flemish Dual Publication System. The Crossover Genre 'Humoristic Adventure." In From Comic Strips to Graphic Novels: Contributions to the Theory and History of Graphic Narrative, 255-69. Eds. Daniel Stein and Jan-Noël Thon. Berlin and Boston, MA: Walter de Gruyter.

Livingston, Sonia. 1998. Making Sense of Television. The Psychology of Audience Interpretation. 2nd ed. London and New York: Routledge.

Louwerse, Max and Don Kuiken. 2004. "The Effect of Personal Involvement in Narrative Discourse.” Discourse Processes 38.2: 169-72.

Lyons, John. 1977. Semantics. Volume 2. Cambridge: Cambridge University Press.

Magliano, Joseph P. and Jeffrey M. Zacks. 2011. "The Impact of Continuity Editing in Narrative Film on Event Segmentation." Cognitive Science 35: 1489-517.

Margolin, Uri. 1995. "Characters in Literary Narrative: Representation and Signification.” Semiotica 106: 373-92. 
2005. "Character." In Routledge Encyclopedia of Narrative Theory, 52-57. Eds. David Herman, Manfred Jahn and Marie-Laure Ryan. New York: Routledge.

—. 2007. "Character." In The Cambridge Companion to Narrative, 66-79. Ed. David Herman. Cambridge: Cambridge University Press.

Marion, Philippe. 1993. Traces en cases. Travail graphique, figuration narrative et participation du lecteur. Essai sur la bande dessinée. Louvain-la-Neuve, Belgium: Université Catholique de Louvain: Academia.

McCloud, Scott. 1993. Understanding Comics: The Invisible Art. Northampton, MA: Kitchen Sink Press.

- 2000. Reinventing Comics. New York: Perennial/HarperCollins.

McHale, Brian. 1978. "Free Indirect Discourse: A Survey of Recent Accounts." PTL: A Journal for Descriptive Poetics and Theory of Literature 3.2: 249-87.

- 1993. "Whatever Happened to Descriptive Poetics?" In The Point of Theory. Practices of Cultural Analysis, 56-65. Eds. Mieke Bal and Inge E. Boer. Amsterdam: Amsterdam University Press.

Meesters, Gert. 2010. "Les significations du style graphique.” Textyles, revue des Lettres belges de langue française 36-7: 519-26.

Meister, Jan C. 2015. "Narratology." In The Living Handbook of Narratology. Eds. Peter Hühn, John Pier, Wolf Schmid and Jörg Schönert. Hamburg: Hamburg University. http://wikis.sub.uni-hamburg.de/lhn/index. php/Narratology.

Meskin, Aaron. 2007. "Defining Comics?" The Journal of Aesthetics and Art Criticism 65.4: 369-79.

Miall, David S. and Don Kuiken. 1994. "Foregrounding, Defamiliarization, and Affect Response to Literary Stories.” Poetics 22: 389-407

Mikkonen, Kai. 2008. "Presenting Minds in Graphic Narratives." Partial Answers 6.2: 301-21.

- 2010. "Remediation and the Sense of Time in Graphic Narratives." In The Rise and the Reason of Comics and Graphic Literature. Critical Essays on the Form, 74-86. Eds. Joyce Goggin and Dan Hassler-Forest. Jefferson, NC: McFarland Press.

- 2011a. "The Implicit Narrator in Comics. Transformations of Free Indirect Discourse in Two Graphic Adaptations of Madame Bovary." International Journal of Comic Art 13.2: 473-87.

- 2011b. "Graphic Narratives as a Challenge to Transmedial Narratology: The Question of Focalization.” American Studies/Amerikastudien 56.4: 639-45.

- 2012. "Focalisation in Comics. From the Specificities of the Medium to Conceptual Reformulation." Scandinavian Journal of Comic Art 1: 69-95.

—. 2013. "Subjectivity and Style in Graphic Narratives." In From Comic Strips to Graphic Novels: Contributions to the Theory and History of Graphic Narrative, 101-26. Eds. Daniel Stein and Jan-Noël Thon. Berlin and New York: Walter de Gruyter/Narratologia Series.

Miller, Ann. 2007. Reading Bande Dessinée: Critical Approaches to French-Language Comic Strip. Bristol: Intellect Books.

Miodrag, Hannah. 2013. Comics and Language: Reimagining Critical Discourse on the Form. Jackson: University Press of Mississippi.

MODE. 2012. Glossary of Multimodal Terms. https://multimodalityglossary. wordpress.com/. 
Morgan, Harry. 2009. "Graphic Shorthand: From Caricature to Narratology in Twentieth-Century Bande dessinée and Comics." European Comic Art 2.1: 21-39.

Nelles, William. 1990. "Getting Focalization into Focus.” Poetics Today 11: 363-82.

Nikolajeva, Maria and Carole Scott. 2001. How Picturebooks Work. New York and London: Garland Publishing.

Nodelman, Perry. 1991. "The Eye and the I: Identification and First-Person Narratives in Picture Books.” Children's Literature 19: 1-30.

O’Neill, Patrick. 1992. "Points of Origin: On Focalisation in Narrative." Canadian Review of Comparative Literature/Revue Canadienne de Littérature Comparée 19: 331-50.

Oswalt, Robert L. 1994. "Inanimate Imitatives in English.” In Sound Symbolism, 293-306. Eds. Leanne Hinton, Johanna Nichols and John J. Ohala. Cambridge: Cambridge University Press.

Palmer, Alan. 2004. Fictional Minds. Lincoln: University of Nebraska Press.

- 2010. Social Minds in the Novel. Columbus: Ohio State University Press.

Patron, Sylvie. 2009. Le narrateur. Introduction à la théorie narrative. Paris: Armand Collin.

Peeters, Benoît. 1991. Case, planche, récit: comment lire une bande dessinée. Tournai: Casterman.

1998. Lire la bande dessinée. Paris: Flammarion.

2007. "Four Conceptions of the Page." Trans. Jesse Cohn. ImageTexT: Interdisciplinary Comics Studies 3.3. http://www.engl...eeters/index.shtml.

Persson, Per. 2003. Understanding Cinema: A Psychological Theory of Moving Imagery. Cambridge: Cambridge University Press.

Phelan, James. 1989. Reading People, Reading Plots. Character, Progression, and the Interpretation of Narrative. Chicago and London: The University of Chicago Press.

- 1996. Narrative as Rhetoric. Technique, Audiences, Ethics, Ideology. Columbus: Ohio State University Press.

- 2005. Living to Tell about It. A Rhetoric and Ethics of Character Narration. Ithaca, NY and London: Cornell University Press.

- 2007. Experiencing Fiction: Judgments, Progressions, and the Rhetorical Theory of Narrative. Columbus: The Ohio State University Press.

Phelan, James and Peter J. Rabinowitz. 2012. "Character." In Narrative Theory: Core Concepts and Critical Debates, 111-18. Eds. David Herman, James Phelan, Peter J. Rabinowitz and Robyn Warhol. Columbus: The Ohio State University Press.

Postema, Barbara. 2013. Narrative Structure in Comics. Making Sense of Fragments. Rochester, NY: RIT Press.

Potsch, Elisabeth and Robert F. William. 2012. "Image Schemas and Conceptual Metaphor in Action Comics." In Linguistics and the Study of Comics, 13-36. Ed. Frank Bramlett. London: Palgrave Macmillan.

Pound, Richard. "'The Gallery of Comicalities': An Introduction to the Caricatures of C. J. Grant, (flu. C1830-1846).” http://www.gjsaville-caricatures. co.uk/articles/GJGrant (originally published in Graham Saville's Caricature Catalogue 25, 1998). 
Prince, Gerald. 1992. Narrative as Theme. Studies in French Fiction. Lincoln and London: University of Nebraska Press.

- 2003a. Dictionary of Narratology. Lincoln: University of Nebraska Press.

. 2003b. "Surveying Narratology." In What Is Narratology? Questions and Answers Regarding the Status of a Theory, 1-16. Eds. Tom Kindt and Hans-Harald Müller. Berlin: Walter de Gruyter.

Rabkin, Eric S. 2009. "Reading Time in Graphic Narrative." In Teaching the Graphic Novel, 36-43. Ed. Stephen E. Tabachnick. New York: The Modern Language Association of America.

Richardson, Brian. 1997. Unlikely Stories. Causality and the Nature of Modern Narrative. Newark: University of Delaware Press.

Rimmon-Kenan, Shlomith. 2003. Narrative Fiction. Contemporary Poetics. 2nd ed. London and New York: Routledge.

Rommens, Aarnoud. 2000. "Manga Story-Telling/Showing." Image and Narrative 1. http://www.imageandnarrative.be/inarchive/narratology/aarnoudrommens. htm.

Round, Julia. 2007. "Visual Perspective and Narrative Voice in Comics: Redefining Literary Terminology." International Journal of Comics Art 9.2: $316-29$.

- 2013. "Anglo-American Graphic Narrative." In From Comic Strips to Graphic Novels: Contributions to the Theory and History of Graphic Narrative, 325-45. Eds. Daniel Stein and Jan-Noël Thon. Berlin and New York: Walter de Gruyter.

Ruusuvuori, Johanna and Anssi Peräkylä. 2009. "Facial and Verbal Expressions in Assessing Stories and Topics." Research on Language and Social Interaction 42.4: 377-94.

Ryan, Marie-Laure. 2004. “Introduction.” In Narrative across Media, 1-40. Ed. Marie-Laure Ryan. Lincoln: University of Nebraska Press.

—. 2005. "On the Theoretical Foundations of Transmedial Narratology." In Narratologia: Narratology Beyond Literary Criticism: Mediality, Disciplinarity, 1-23. Berlin: Walter de Gruyter.

- 2006. Avatars of Story. Minneapolis: University of Minnesota Press.

. 2013. "Transmedial Storytelling and Transfictionality." Poetics Today 34.3: 361-88.

- 2015. "Narration in Various Media." In The Living Handbook of Narratology. Eds. Peter Hühn, John Pier, Wolf Schmid and Jörg Schönert. Hamburg: Hamburg University. http://wikis.sub.uni-hamburg.de/lhn/index. php/Narration_in_Various_Media.

Ryan, Marie-Laure and Jan-Noël Thon. 2014. "Storyworlds across Media. Introduction." In Storyworlds across Media: Toward a Media-Conscious Narratology, 12-22. Eds. Marie-Laure Ryan and Jan-Noël Thon. Lincoln: University of Nebraska Press.

Sadrô, Javid, Izzat Jarudi and Pawan Sinha. 2003. "The Role of Eyebrows in Face Recognition.” Perception 32.3: 285-93.

Saraceni, Mario. 2001. "Relatedness: Aspects of Textual Connectivity in Comics.” In The Graphic Novel, 167-79. Ed. Jan Baetens. Leuven: Leuven University Press.

- 2003. The Language of Comics. London and New York: Routledge.

Sattler, Peter R. 2010. "Past Imperfect: 'Building Stories' and the Art of Memory." In The Comics of Chris Ware: Drawing is a Way of Thinking, 
206-22. Eds. David M. Ball and Martha B. Kuhlman. Jackson: University Press of Mississippi.

Schüwer, Martin. 2008. Wie Comics erzählen. Grundriss einer intermedialen Erzähltheorie. Trier: Wissenschaftlicher Verlag Trier.

Sellors, C. Paul. 2007. "Collective Authorship in Film.” The Journal of Aesthetics and Art Criticism 65.3: 263-71.

Semino, Elena. 2011. "Deixis and Fictional Minds.” Style 45.3: 418-40.

Shinohara, Kazuko and Yoshihiro Matsunaka. 2009. "Pictorial Metaphors of Emotion in Japanese Comics.” In Multimodal Metaphor, 265-93. Eds. Eduardo Urios-Aparisi and Charles Forceville. Berlin: Walter de Gruyter.

Singer, Marc. 2012. "Time and Narrative: Unity and Discontinuity in The Invisibles." In Critical Approaches to Comics. Theories and Methods, 55-70. Eds. Matthew J. Smith and Randy Duncan. New York and London: Routledge.

Sinha, Pawan, Benjamin Balas, Yuri Ostrovsky and Richard Russell. 2005. "Face Recognition by Humans: 20 Results all Computer Vision Researchers Should Know About." http://web.mit.edu/bcs/sinha/papers/20Results_ 2005.pdf.

Smith, Greg M. 2003. Film Structure and the Emotion System. Cambridge: Cambridge University Press.

Smith, Murray. 1995. Engaging Characters: Fiction, Emotion, and the Cinema. Oxford: Clarendon.

Smolderen, Thierry. 2002. "Ceci n'est pas une bulle! Structures énonciatives du phylactère." In Actes des Quatrièmes Rencontres Réseaux Humains/Réseaux Technologiques, 107-39. Eds. Eric Espéret and Jean-François Cerisier. Poitiers: CNDP. http://rhrt.edel.univ-poitiers.fr/document.php?id=555.

- 2009. Naissances de la bande dessinée. De William Hogarth à Winsor McCay. Bologne: Les Impressions Nouvelles.

- 2012a. "Les bandes dessinées du Graphic et de l'Illustrated London News." Neuvième Art 2.0. http://neuviemeart.citebd.org/spip.php?rubrique71. - 2012b. "Histoire de la bande dessinée: questions de méthodologie." In La bande dessinée: une médiaculture, 71-90. Eds. Éric Maigret and Matteo Stefanelli. Paris: Armand Colin.

- 2014a. Origins of Comics: From William Hogarth to Winsor McCay. Jackson: University Press of Mississippi.

- 2014b. "Graphic Hybridization, the Crucible of Comics." In The French Comics Theory Reader, 47-61. Eds. Ann Miller and Bart Beaty. Leuven: Leuven University Press/Studies in European Comics and Graphic Novels.

Stanzel, F.K. 1984. A Theory of Narrative. Trans. Charlotte Goedsche. Cambridge: Cambridge University Press.

Stöckl, Hartmut. 2004. "In Between Modes: Language and Image in Printed Media.” In Perspectives on Multimodality, 9-30. Eds. Eija Ventola, Cassily Charles and Martin Kaltenbacher. Amsterdam: John Benjamins Publishing Company.

Surdiacourt, Steven. 2012. "Tying Ends Together: Surface and Storyworld in Comics.” Comics Forum. http://comicsforum.org/2012/12/27/image-narrative8-tying-ends-together-surface-and-storyworld-in-comics-by-steven-surdiacourt/. - 2015. Comics and Storytelling. Towards a Mediumspecific Narratology. PhD dissertation. Katholieke Universiteit Leuven Faculteit Letteren. 


\section{Bibliography}

Swift, Jonathan. 1736/1998. "A Character, Panegyric, and Description of the Legion Club.” In Verse in English from Eighteenth-Century Ireland, 222-30. Ed. Andrew Carpenter. Cork: Cork University Press.

Talmy, Leonard. 2000. Toward a Cognitive Semantics. Cambridge, MA: MIT Press.

Tan, Ed S. 2001. “The Telling Face in Comics Strip and Graphic Novel.” In The Graphic Novel, 31-46. Ed. Jan Baetens. Leuven: Leuven University Press.

Thomson-Jones, Katherine. 2007. "The Literary Origins of the Cinematic Narrator." British Journal of Aesthetics 47.1: 76-94.

Todorov, Tzvetan. 1969. Grammaire du Décaméron. The Hague: Mouton.

Tomashevsky, Boris. 1965. "Thematics.” In Russian Formalist Criticism: Four Essays, 61-95. Eds. Lee T. Lemon and Marion J. Reis. Lincoln and London: University of Nebraska Press.

Tondro, Jason. 2015. "Nightcrawlers' Inferno and Other Hellish Tales: Comics Adaptations of Dante." In Medieval Afterlives in Contemporary Culture, 291-300. Ed. Gail Ashton. London: Bloomsbury Publishing.

Töpffer, Rodolphe. 1845/1944. "Essai de physiognomonie.” In Töpffer, l'invention de la bande dessinée, 185-225. Eds. Thierry Groensteen and Benoît Peeters. Paris: Hermann.

Uidhir, Christy M. 2014. "Comics and Collective Authorship." In The Art of Comics. A Philosophical Approach, 47-67. Eds. Aaron Meskin and Roy T. Cook. Chichester: Wiley Blackwell.

Van Lier, Henri. 1988. "La bande dessinée, une cosmogonie dure." In Bande dessinée, récit et modernité, 5-24. Ed. Thierry Groensteen. Paris: Colloque de Cerisy.

Verstraten, Peter. 2009. Film Narratology. Trans. Stefan van der Lecq. Toronto: University of Toronto Press.

Walker, Mort. 2000. The Lexicon of Comicana. Lincoln, NE: Authors Guild Backinprint.com Edition.

Walton, Kendall L. 1997. "On Pictures and Photographs. Objections Answered.” In Film Theory and Philosophy, 60-75. Eds. Richard Allen and Murray Smith. Oxford: Clarendon Press.

Waugh, Colton. 1947/1991. The Comics. Jackson: University Press of Mississippi.

Widiss, Benjamin. 2013. "Comics as Non-sequential art. Chris Ware's Joseph Cornell." In Drawing from Life: Memory and Subjectivity in Comic Art, 86-111. Ed. Jane Tolmie. Jackson: University Press of Mississippi.

Witek, Joseph. 2009. "The Arrow and the Grid: Creating the Comics Reader." In A Comics Studies Reader, 149-56. Eds. Jeet Heer and Kent Worcester. Jackson: University Press of Mississippi.

Wolf, Werner. 2003. "Narrative and Narrativity: A Narratological Reconceptualisation and Its Applicability to the Visual Arts." Word \& Image: A Journal of Verbal/Visual Enquiry 19.3: 180-97.

Wolk, Douglas. 2007. Reading Comics. How Graphic Novels Work and What They Mean. Philadelphia, PA: Da Capo Press.

Woloch, Alex. 2003. The One vs. the Many. Minor Characters and the Space of the Protagonist in the Novel. Princeton, NJ and Oxford: Princeton University Press. Yannicopoulou, Angela. 2010. "Focalization in Children's Picture Books." In Telling Children's Stories: Narrative Theory and Children's Literature, 65-85. Ed. Mike Cadden. Lincoln: University of Nebraska Press. 


\section{Index}

Ada (by Altan) 231

affordance 19-20, 23, 29n27, 220

alignment 102-06

Ally Sloper (magazine) 274n 4

Altman, Rick 102-03, 105-06

angle 7, 38, 42, 94-6, 169, 234, 254,

274n8; eyeline image 165-67;

(page) bleed 57, 234; close up 54,

$79,92,101,169,210,223-24,234$,

252-53; over-the-shoulder image

94, 165-66, 168; panorama 101,

$231,250,268,269$; reaction image

166, 168, 223; reverse 92, 139, 168

'arthrology' 42-3, 68n14

Ascension du Haut Mal (Epileptic)

(by David B) 141

Asterios Polyp (by David

Mazzucchelli) 120, 123, 125n11, 154-55

Asterix (by Albert Uderzo and René

Goscinny) 137, 227, 230, 233,

236-37; Asterix and the Banquet 232

authorship in comics 130, 148n2; implied author 6, 134-36

autobiographical comics 10, 17, 23, 99, 124n $3,130,132-33,137$, 140-42, 161, 220, 227

Baetens, Jan 5, 8-9, 40, 68n16, 69n23, 70n35, 70n43, 88n6, 89n14, 89n16, 108n16, 149n15, 241n7

Bal, Mieke 4, 28n6, 151, 171, 173n9, 184, 198n6, 210, 218

Bande pas dessinée (by Bruno Muschio) 231

Barefoot Gen (by Keiji Nakazawa) 141

Bateman, H.M. 79-81

Beaty, Bart 13-14, 29n22, 41, 68n12

Berlin (by Jason Lutes) 207
Blake and Mortimer (by Edgar

P. Jacobs) 67

Blast (by Manu Larcenet) 120-23

Booth, Wayne C. 76

Bordwell, David 4, 93, 110, 136, 148n7, 148n8

Branigan, Edward 4, 70n38, 167, 173n13, 173n17

Bretécher, Claire 228

Bridgeman, Teresa 101, 107n2

broadsheet print $228,246-47,249$, 251, 253, 268, 272-73

Brooke-Rose, Christine 53, 56

Brooklyn Station Terminus Cosmos (by Jean-Claude Mézières and Pierre Christin) 239

Bugs Bunny (comic) 1

Building Stories (by Chris Ware) 33-35, 38, 43, 49, 50, 51, 63, 65, $67,67 \mathrm{n} 2,150,176$

Cage, The (by Martin Vaughn-James) 174, 175-76

Calvin and Hobbes (by Bill Watterson) $6,23,112,171$

caption 54, 56, 62, 92, 97, 105, 107n6, $176,220,228,232,236,249-50$, 252, 265, 266, 267; lyrical 175-76; narrative 132, 134, 144, 232, 236

cardinal function (in plot) 58, 59, 70 n37

caricature $64,246,247,248,249$, $250,251,252,253,256,257,258$, $170,272,273,275 \mathrm{n} 20$; and reality 164, 198; art of 195, 197, 246, 248; as a form of perception 219; as style 114-15, 198; bodily forms 222 , 224; characters $179,180,186,188$, 195, 197, 199n 20; Golden Period of $27,251,256,272$ 
caricature magazine $27,246,247$, $249,251,252,256,260,264,269$, 271, 272-73

Carroll, Noël 19

cartoon $10,12,18,77,87,88 \mathrm{n} 5$, $88 \mathrm{n} 6,228,247-47,250,273$, $274 \mathrm{n} 4$; as style $6,101,195$

character: allegorical 179, 189-90, 193, 199n20; and action 178-79; as an individual 119-20, 122, 123, 156, 158, 185, 186, 189, 190-92, 195, 197, 199n15, 217, 261; as means of narrative continuity 90-107; basis type 179-80, 184; complex 178, 186-93, 199n20, 246; concept of 177 ; continuing presence 90-91, 183; flat 186, 188, 189, 199n17; mental state 36-37, 50, 84, 91, 96, 120, 123, 176, 179-80, 183, 185, 186, 188, 190, 191, 195, 196-97, 225-28, 239, 241n12; mimetic function (or dimension) $107,107 \mathrm{n} 1,107 \mathrm{n} 3$, 178, 179, 180, 193, 199n8; movement of 94, 96-100; reflecting $150,219 \mathrm{n} 8$; round 186,188 , 199n17; shift 96, 108n12; synthetic function (or dimension) 81, 91, 106, 107n3, 178, 193, 199n8; thematic function (or dimension) $107 \mathrm{n} 3$, 178, 179, 185, 187, 192, 193, 199n8; type-like 186, 188, 189 , 190, 192, 197

characterisation in comics $8,9,37$, 50, 114, 120, 174-98, 199n13, 233; definition of 177-78; principles of 184-85

Chat du rabbin, Le (The Rabbi's Cat) (by Joann Sfar) 113, 207

Chatman, Seymour 4, 6-7, 28n9, 53, 134-35, 148n1

Chavanne, Renaud 68n22, 107n11

Chute, Hilary 17

'closure' 38-40, 42, 68n10

Clumsy (by Jeffrey Brown) 49

Cohan, Steven 6

Cohn, Dorrit 205-06

Cohn, Jesse 68n21, 69n24

Cohn, Neil 68n10, 68n17, 69n27

colour: characterisation 190; emotional state 229; means of layout 54; perspective 150, 154, 167, 169, 230; salience 101, 230; stylistic effect 70n39, 83, 120-122, 125n15; stylistic marker 42;

subjectivity $104,109,120,215 \mathrm{n} 15$

comic strips 6-7, 24, 66-67, 94, 132, 220, 231, 246, 247-50, 262, 264, $272,274 \mathrm{n} 6,275 \mathrm{n} 13$

comics: concept of $12-15,247,274 n 6$

Commando (comic) 99

connectivity 38, 45-48, 63, 81, 91-92, 95, 102, 106, 123, 233, 258

constraint: artistic (formal) 20-23, 29n29, 29n30; medium-specific 8, 156, 203, 204, 211, 217, 220, 237-38

continuing-consciousness frame 122 , 125n14, 137, 215, 217

Corto Maltese (by Hugo Pratt) 58-59, 195, 200n24, 208-09; La Ballade de la mer salée (The Ballad of the Salty Sea) 206; Les Celtiques (Celtic Tales) 58

Cruikshank, George 251, 253, 254, 256, 257, 275n 23

Cruikshank, Robert 269

Cutting, James E. 108n12, 108n14

Dante (Alighieri) 180, 183, 185, 189; Inferno (from The Divine Comedy) 180, 181, 182, 189, 194, 200n23

Dark Night Returns, The (by Frank Miller) 193

deixis 120, 152, 155, 156-57, 160, $170,172,172 \mathrm{n} 1,199 \mathrm{n} 12$

Delany, Samuel R. 10, 28n16

Delisle, Guy 58-59, 141, 215

Dessous Troublants (by Jeanne Puchol) 176

dialogue in comics 220-40; and action 96-97, 137, 207, 220; bond between speaker and utterance 228-32; characterisation 92, 176, 185, 208, 217; conversational scene 53, 76, 94-95, 134, 144, 168, 188, 220, 224-26, 227-28, 230, 231, 232, 233-36, 238-39, 240; duration 53, 253; embodied speech situation 221-25, 239; fundamental narrator 133-34, 144; gaze 222, 223, 227-28, 234, 241n6; narrative function 232-38, 238-39; non-verbal communication 222-25; $180^{\circ}$ rule $107 \mathrm{n} 10$; repetition 62 ; showing 76

Dickens, Charles 186, 199n16, 251 diegesis and mimesis 76, 88n9 
Distant Neighborhood, A (by Jiro Taniguchi) 24, 137-48

Donald Duck (comic) 1

Donaldson-Evans, Mary 209-10 duration, see time in comics

Dürrenmatt, Jacques 29n30, 89n13, $241 \mathrm{n} 12$

editing techniques 54, 81, 92-93, 107n5, 131, 167; eyeline match 165-67, 169, 216; match cut (or graphic cut/match) 41, 93, $107 \mathrm{n} 5,165-67$; match on action 92-96, 108n13

Eisner, Will 29n25, 141, 234-35, $242 \mathrm{n} 22$

emanata 109, 225-27, 239, 241n11

Ethel \& Ernest. A True Story (Raymond Briggs) 85

Evens, Brecht 228, 230

Every Body's Album and Caricature Magazine 251, 258, 259, 266, 269, 272, 273

facial expression 47, 80, 84, 97, 167 , 184, 196, 205, 241n10; conversation 27, 222-25, 227, 239; deep structure of visual storytelling 22-23

"Feinte Trinité" (by François Ayroles) 231

film narrative 54, 81, 93, 134, 154, $157,161,167,203,224$

film studies 4, 9, 11, 25, 110, 129, 131, 136, 147, 148n 3, 167, 173n17, 203-04, 241n6

Fishelov, David 188-93

Flaubert, Gustave 209, 212-13, 215

Fludernik, Monika 29n24, 152-53, $154,155,203,219 \mathrm{n} 11$

focalisation $3,5,8,28 \mathrm{n} 11,37,49$, 91, 103, 150-72; authorial 140, 217; cognitive 120, 152, 157-60; definition of 150-52; direct gaze 167; embedded (or simultaneous) 6, 171, 173n18, 210; external 6, $151,165,219 \mathrm{n} 5$; focaliser 26,132 , 139, 143, 147-48, 151-52, 154, 158, 160-65, 171, 172, 173n11, 210, 218; focalised 91, 147-48, 151-52, 155-56, 171-72, 217; gaze image $116,153,165-68,170$, 223; impersonal 165; internal 49, 139, 142, 149n16, 151, 165, 212; ocularisation $8,157-60,173 \mathrm{n}$; perceptual 158-60, 170, 171; point-of-view image 138-44, 151, 159-60, 165-66, 168, 173n13; perception image 166-67; zero-level 139, 147, 151; see also angle

following-unit, see alignment

Forceville, Charles 168, 210, 230, 241n13, 241n15, 241n18, $242 n 23$

Forster, E. M. 186, 188, 199n17

frame $7,22,38,44-45,65,160-64$, 230; broken $85,108 \mathrm{n} 17,145$, 233-34, 270-71; embedded 45-46; expressive function $87,110,113$, $117,142,144,145,166,168,206$, 240, 262; frame of vision 218; key frame 57-60; hors-champ (off panel space) 88,89 n 16,157 ; rhythm 54 , $69 \mathrm{n} 32$, salience $70 \mathrm{n} 39$; see also multiframe

Fred (Frédéric Othon Théodore Aristidès) 23, 47, 51, 57, 112, 119; Philémon 47, 51-2, 57, 112

free indirect discourse (FID) 28n14, 168, 204, 209-14, 217, 220

free indirect perception 168, 203, 211

Fresnault-Deruelle, Pierre 5, 36, 60

Frey, Hugo 40, 69n23, 88n6, 108n16, 149n15

Fun Home (by Alison Bechdel) 17, 141

Gaudreault, André 4, 5, 81-83, 88n8, $88 \mathrm{n} 9,124 \mathrm{n} 2,131,134,148 \mathrm{n} 8$, $149 \mathrm{n} 17$

Gemma Bovery (by Posy Simmonds) 194, 209, 214-15

Genette, Gérard: characterisation 177, 198n6, 199n7; discourse-time 36, 37, 69n31; film narratology 4; focalisation $5,87,139,150-51$, 155, 157, 160-61, 172n3, 172n7, 173n10, 173n18; frequency 60-61; global (or synchronic) look 33, $35,36,66$; metalepsis $69 \mathrm{n} 30$, 138; mimesis 76,156 ; narrative mood 28n11; narratology 3, 8, 83; narrator 5, 87, 149n10,149n13; paralepsis 215; rhythm 52-3, 56; story-time 37 ; temporal order 37 , 68n6, 69n29

genre 18, 23, 65-67, 70n44, 99, 106, 109, 111, 137, 157, 167, 177, 193-96, 245-46, 278

Gibson, James J. 29n27 
Gillray, James 251, 252, 256, 257, 258, 268, 272, 274n8, 275n16

Glasgow Looking Glass (magazine) 251, 269, 272, 274n11, 274n12 global look 33, 36, 40, 46, 64-66, $181,260,269$; see also reading Gorey, Edward 199n14

Grant, Charles Jameson 251, 258-59, 266, 272-73, 274n11

graphiation, see graphic style graphic cut, see editing techniques graphic match, see editing techniques graphic narrative: definition of 17 graphic novel 8, 10, 17, 29n25, 40, 67, 88n6, 94, 99, 108n16, 124, 144, 149n15, 161, 186, 194-95, 217-18, 245-46, 271

graphic style $9,11,47,75,86-87$, 109-24, 124-125n7, 147, 184, 217, 246-47; cartoony $101,114-15$, 195-96; definition of 110-13; graphiation 86-87, 88n12, 88n13, 88n14, 131-32; ligne Claire 113, 115; mind style 119-24; narrative function $11,47,86,87,111-12$, 115-19, 119-23; photorealism 75, 115, 148, 195-196, 198; rupture 47, 115-19, 120-23, 125n12, 147; variation 115-19; see also writing

Groensteen, Thierry 5, 42; artistic constraints 21; bipolar structure between speech balloons and characters 230, 240; braiding 42-43; character 198; definition of comics 12, 29n 21 ; frames 69n32; foyer perceptif 173; fundamental narrator 112, 131-33, 149n11; iconic solidarity $12,28 \mathrm{n} 12,42$, $56,68 \mathrm{n} 20$; layout style 69n23; multicouche 157; multiframe 43, 45 ; narrative drawing $77,88 \mathrm{n} 2$; narratology $5,28 \mathrm{n} 8$; repetition $70 \mathrm{n} 41$; rhythmic function of speech balloons $242 \mathrm{n} 26$; salience $70 n 39$; string vs. sequence $274 \mathrm{n} 7$; stylistic variation $115-16$; see also 'arthrology' and multiframe

Hagelberg, Matti 1, 43-44

Harvey, Robert C. 68n11, 111

Hatfield, Charles 57, 70n35, 125n11

Heath, William 251, 252, 253, 255, $256,261,262,263,268,272,273$, $274 \mathrm{n} 11,275 \mathrm{n} 17$
Here (by Richard McGuire) 46

Hergé (Georges Prosper Remi) 46, 107n8, 113, 125n8, 186, 195, 205

Herman, David 8, 16, 28n4, 57-58, $63,70 \mathrm{n} 42,198 \mathrm{n} 5,200 \mathrm{n} 27$

historical analysis of comics 10-11, 105-06, 245-51, 278; see also narratology, diachronic

Hogarth, William 247, 248, 250, 256, 265

image: concept of 78-79

image and word interplay 13-15, 64-65, 75, 120, 147, 153-54, 156, 184, 196-7, 204, 215-16, 219, 221, $232,238-39$

imitative 221, 226, 240n2

implied author, see authorship in comics

Intérieurs (by Régis Franc) 174-75

intermediality 3-4, 15, 19, 28n5

"I Want You to Like Me" (Martin Cendreda) 101, 231-32

Jahn, Manfred 135, 148n6, 155, 166

Jannidis, Fotis 177, 179-80

Jimbo's Inferno (by Gary Panter) 180-85, 189-90, 199n10, 199n11

Jimmy Corrigan: The Smartest Kid on Earth (by Chris Ware) 150

Jost, François 4, 88n11, 124n2, $148 \mathrm{n} 8,157-60,170$

juxtaposition, see narrative organisation

Kindt, Tom 9-10

Kirby, Jack 57, 130, 195

Kress, Günther 27n3, 29n27, 101, 167

Kukkonen, Karin 8, 107n10

Kunzle, David 108n17, 245, 250, 264, $274 \mathrm{n} 7,275 \mathrm{n} 13$

Lagoon, The (by Lilli Carré) 238

League of Extraordinary Gentlemen, The (by Alan Moore and Kevin O’Neill) 74

Leech, Geoffrey 48-50, 119-20

Leech, John 77-78, 247

van Leeuwen, Theo 27n3, 101, 167

Lefèvre, Pascal 89n16, 124-125n7, 242n19, 273n1

Léo (Luiz Eduardo de Oliveira) 74-75

Lichtenstein, Roy 60 
literary narrative: adaptation 196-96; characterisation 184, 194, 197, 218; dialogue 224; diegetic level 142, 238; focalisation $147,151,154$, 155-56, 159, 161, 165, 171, 172n3, 218; narrator $82,148 \mathrm{n} 4$; showing 75-76; speech and thought 206, 209-12, 217; time 50-51, 52, 209, 238-89; utterance 229

literary value in comics 13-16, $28 \mathrm{n} 18$

Little Nemo (by Winsor McCay) 46, 112

"logophobia" 14, 29n20

Madame Bovary (by Daniel Bardet and Michel Janvier) 209-13, 219n6

Marey effect 156-57, 172n6

Margolin, Uri 185, 198n6

Marion, Philippe 5; colour effects 122; continuity 45-48; graphic enunciation 82-83, 131-33; graphisme 78; "mega-narrator" 112, 131-33; monstration 86, 89n14, 131; narrative and painterly functions of a panel 60, 70n40; stylistic rupture 119

Marshall Blueberry (Le Spectre aux balles d'or/The Ghost with the Golden Bullets) (by Jean-Michel Charlier and Jean Giraud) 206-08

match cut, see editing techniques

match on action, see editing techniques

Maus (by Art Spiegelman) 17

McCloud, Scott 12, 29n20, 38-9, 41-42, 67n1, 69n29, 195; The Sculptor 54, 167

medium: definition of 17-18; comics as a medium $7-8,9,13,15-18,20$, 100, 131, 159, 188, 247; see also medium-specificity

medium-specificity $3,6-7,8,9,11$, $12,18-23,81,86-88,95,106,123$, 129-30, 148, 152, 157, 166, 171-2, 177, 194-95, 203, 211-12, 218, 220-21, 224, 238, 240, 277

metalepsis 51, 69n30, 138, 142

Meti (by Aapo Rapi) 227-28, 238

Mickey's Inferno (L'Inferno di Topolino) (by Guido Martina and Angelo Bioletto) 180-83, 185-87, 189, 191-92, 195, 197, 200n26
Miller, Ann 8

mind style, see graphic style

Miodrag, Hannah 14, 28n19, 29n20, $68 \mathrm{n} 17,111,124 \mathrm{n} 6$

mise en page, see page layout

Mr. Block (by Ernest Riebe) 183

"Mr Spoonbill's Experiences in the Art of Skating" (by John Tenniel) 99-100

Mister Wonderful (by Daniel Clowes) 232, 236, 238

Monsieur Vieux Bois (by Rodolphe Töpffer) 205

monstration, see showing

Morgan, Harry 5, 107n2, 200n25

Mother, Come Home (by Paul Hornschemeier) 216

Motherless Oven, The (by Rob Davis) 94

multiframe (multicadre) 43-45, 68n19 multimodality 2, 9, 13, 14, 18, 19, 22-23, 27n3, 29n27, 64, 91-92, 153, 154, 238-39; characterisation 192, 197; focalisation 160-161; multimodal document 27n3, 144; speech representation 203-04, 209, 211, 218-19, 221-22

Müller, Hans-Harald 9-10

Nao of Brown, The (by Glyn Dillon) 190-91, 194, 197

narrative: behaviourist 208-09, $217,218,219$ n 5 ; causal cohesion 90-1; concept of $15-16$; first-person 49, 92, 101, 124, 130, 132, 137, 143-45, 146, 147, 149n13, 149n16, 161, 170, 175, 179, 189, 192, 193-94, 196, 204, 207, 214, 215-16, 218, 220, 232; it-narrative 174-76, 198n1; levels 123, 138, 145, 160; third-person 106, 204, 205 , 207-09, 215, 217, 218; visual 4, 7, 36, 54, 151, 153, 157, 159, 171, 210-11, 217-18

narrative agency $83,124,129-31$, 135-36, 138, 139, 141-42, 143, 144, 147-48; see also narrator narrative breakdown 24, 28n15, 42, 48, 55, 65, 68n11, 131, 206

narrative organisation: juxtaposition 40, 47, 205, 252-60; sequentiality 22, 69n27, 97, 248, 249, 260-68, 272, 273, 275n23; simultaneity 


\section{Index}

154-55, 248, 249, 268-73; see also sequencing

narrative salience, see salience narrativist bias 13-14

narrativity $2,11,16,22,27 \mathrm{n} 1,43$, 45, 90-1, 106, 107n2, 178, 203, 254-55, 273

narratological analysis 9-11, 277-78 narratology 2-11, 15, 16, 22-23, 28n6, 33, 36, 75, 81, 83, 103, 131, 144, 147, 150, 153, 172, 177-78, 203, 245, 277-78; diachronic 105, 246; postclassical 3, 28n4, 152; transmedial 154, 168, 210

narrator in comics 5, 8-9, 16, 29n23, 75-76, 81-83, 112, 123-24, 130-37, 148, 149n11, 150, 153, $158,159,161,165,170,215-16$, 217-18, 220; continuing narratorial voice 143, 204, 206; extradiegetic 210; fundamental 112, 131-36; heterodiegetic 132, 137, 144, 148n1, 149n10, 210; homodiegetic 148, 149n10, 214, 215; impersonal narration 135-37; intradiegetic 132, 148n1; mega-narrator 82, 112, $131,135,137$; narrative voice 36 , 87, 109, 124, 132, 135, 137, 143, 147-48, 152, 154, 207, 251; self-narration 139, 143-44, 216; shown in the image 132-33, 170; see also narrative: first-person

Newton, Richard 251, 252, 256, 258, $275 \mathrm{n} 21$

Nikolajeva, Maria 196

non-fiction comics $17,23,136,139$, 141-42, 161, 164, 176-77, 196, $198,217,225$

non-narrative comics $14,176-77$, 249, 268, 273

Northern Looking-Glass (magazine) $251,253,255,256,272,275$ n 20

ocularisation, see focalisation

Omni-visibilis (by Matthieu

Bonhomme and Lewis Trondheim) 159-60

$180^{\circ}$ rule $9693,96,107 \mathrm{n} 10$

O’Neill, Patrick 6

onomatopoeia 100, 109-10, 149n12, $221,225,226,241 \mathrm{n} 15$

OuBaPO (Ouvroir de bande dessinée potentielle) $21-22,29 \mathrm{n} 30$ page: as a unit of attention and design $38,42,46,47,49,51,64$, 65, 68-69n22, 269-72; double spread $42,47,49,51,57,64,65$, 66, 95-96; splash 57; see also page layout and global look

page layout $10,20,22,24,29 n 30,40$, 42-43, 45, 46-48, 51, 54-56, 61, 64-66, 68n21, 68n22, 70n41, 100, 101, 113, 234, 249, 270-71, 273; layout styles 46-47, 54, 64, 69n23, 69n24, 111, 246

Palestine (by Joe Sacco) 17

panel: as a photograph 79,146 , 161-64; 'memorable panel' 60; narrative function $40,60,70 \mathrm{n} 40$, 146; painterly (or picture) function $40,60,146$; polyptych 57 ; relations 22, 38-43, 45-50, 54, 68n17, 252, 272; wordless $84-85,117,139$, 144-46, 236; see also narrative organisation, panel transitions and time in comics, duration in a single panel, temporality in panel relations

panel transitions 38-43, 92, 96, 160, 274n9; modality-to-modality transition 42,112 ; types of 41-42 paralepsis 215

Peeters, Benoît 46-47, 60, 69n23

Pekar, Harvey 124n3, 141-42

perception image, see focalisation

Persepolis (Marjane Satrapi) 17, 132, 137, 141

perspective: camera-eye 41, 93-94, 140, 142, 155, 156, 158, 167, 168, 173n9, 209, 215, 217; camera movement 54, 129, 158, 168, 169 , 210; spatially determined 152 , 155-57, 169, 172; see also angle and focalisation

Phelan, James 107n3, 178, 193, 199n8, 199n21

Philémon, see Fred

photorealism, see graphic style pictogram 109, 226, 241n13

pictorial rune 225-26, 241n11, $241 \mathrm{n} 13$

picture books 12-13, 18, 146, 153, 161, 165, 196

Pinocchio (by Winshluss) 115-19, 194

Pogo (by Walt Kelly) 227

point-of-view image, see focalisation 
Postema, Barbara 68n15

Prince, Gerald 3, 29n23, 219n5

'progress' story (narrative genre) 250, 256, 260-65, 272, 275n20

Prosopopus (by Nicolas De Crécy) 40-41, 55

publication format $10-11,18,20,47$, 65-66, 83, 245-51, 273, 273n2

Punch (periodical) 77, 79, 98, 99, 247, 251

Pyongyang: A Journey in North Korea (by Guy Delisle) 58-60, 215-16

Qu ..., La (by Marc-Antoine Mathieu) 122

reading: conventions 18,38 , 46, 64-67, 83, 193; 'diegetic absorption' 273; 'tabular' 36, 43, $46,65,66,70 \mathrm{n} 43$; unit of 44,66

repetition: characterisation 184 , 199n13; connectivity 47, 92, 96, 106; iconic 21-22; multiphase image 56-57; salience 63, 70n41, 101; see also connectivity and time in comics, frequency

rhythm, see time in comics

Rimmon-Kenan, Slomith 172n7, 184, 197, 198n6, 199n13, 199n20

Round, Julia 96

Rumble Strip (by Woodrow Phoenix) 175

Ryan, Marie-Laure 3, 27n1, 28n5

Sacco, Joe 17, 141, 242n24

Saga (by Vaughan, Brian K. and Fiona Staples) 92-3

salience: narrative salience $43,58,60$, 91-2, 101-02, 105, 106, 107n4; visual salience $60,101-02,105,106$, $107 \mathrm{n} 4,115$

Sandman, The (by Neil Gaiman et al) 42, 104-06, 227, 231

scene $18,21,38,41,51,52,53-54$, $56,57,68 \mathrm{n} 9,93-96,108 \mathrm{n} 13$, 108n14, 240; see also dialogue, conversational scenes and time in comics, formulas of rhythmic variation

Schüwer, Martin 4-5, 36-37, 67n6

Scott, Mary 196

sequencing $16,48-51,65,77,81$, 125n7, 249; chronological 48; presentational 49-50; psychological 48-49; see also sequential form

sequential art 248

sequential form 196, 248-50, 256, 260-68, 272, 274n7; episodic sequential form 260, 272; narrative continuity sequence $260,264,272$

Seymour, Robert 251, 267, 270, 272, 273, 274n11

Short History of America, The (by Robert Crumb) 174-75

showing 73-89, 131-33, 136-38, 144, 153, 156-57, 214, 215, 219, 221;

continuous showing of character 96-97, 105-06, 217-18; definition of 74; distinction from telling 76-78, 150-51, 217; monstration 81-86, 89n14, 131-35, 144, 149n16, 149n17; narrative function $74,77,78,83$

Shires, Linda M. 6

Short, Mick 48-50, 119-20

simultaneity, see narrative organisation

Smith, Murray 91, 102-03, 105, $108 \mathrm{n} 22,108 \mathrm{n} 23$

Smolderen, Thierry 241n19, 245, 247-49

sound effect, see onomatopoeia spatial attachment, see alignment speech $8,54,55,133-34,177-78$, 203-04, 214, 217-19; direct 76, 77, 192, 207, 208, 213, 214, 217, 219n4, 220, 228; indirect 203, 206, 207, 209, 212-13, 214, 217, 218, 220; inner 203-04, 208-09, 213, 218; monologue 102, 207, 240n1; reported 205-06, 207, 218, 242n20, 267; speech-category approach 203, 204, 217, 219; speech tag 229, 242n20; see also dialogue in comics and free indirect discourse speech balloon $1,13,18,101-02$, 129, 133, 142, 143, 185, 208, 213, 220-21, 228-38, 241-42n19, 248, $249,251,258$; as metaphor for a speech act 229, 240, 243n20; as means of connectivity 233; expressive use 230; para-balloonic phenomena $226,240,241 \mathrm{n} 15$; relation to characters 230-32, 234-36, 237; rhythmic function 232-38

Stanzel, F.K. 213 
strip design 248-50, 262, 264, 265, $266,268,272,273,274 n 7$

style, see graphic style stylistics 48, 110, 115, 119

subjective point of view, see focalisation, point-of-view image and subjectivity

subjectivity 5, 109, 110,165; author 109; cartoonist 82-83, 86, 131, 198; character 109, 146, 156; indicators of $176-77$; narrator 83 , 136,204 ; techniques of subjective focus of perception 165-69; see also focalisation, point-of-view image symbolia 109-110

synchronic reading, see global look and reading

'tabular' reading, see global look and reading

Tamara Drewe (by Posy Simmonds) 188, 192, 197

Taste of Chlorine, $A$ (by Bastien Vivès) 38-39, 49, 61-62, 83

Tenniel, John 97-100

"There Will Come Soft Rains" (by Wally Wood) 176

thought balloon 54, 104, 109, 143, 149n16, 185, 208, 213, 218, 220, $228,229,238$

thought representation $28 \mathrm{n} 14$, 177-78, 188, 204, 211-12, 213, 220; direct 204, 207, 213; reported 76, 206, 207, 208-09, 212, 216, $218,242 \mathrm{n} 20$; see also free indirect discourse

time in comics 3, 5, 8-9, 20, 33-70; 'achronism' 37; anachrony 37-38, $48,51,52$; and genre 66-67; anticipation $37,64,68 \mathrm{n} 8,143$; chronology 16, 33-35, 36, 37, 40, $48,49,50$; duration $20,37,40,45$, 50-58, 62, 99, 206, 226, 229, 233, $235-57,240,253$; duration in a single panel 55-57, 65; ellipsis 18 , 53, 107n6; flashback 34, 37, 38, 41, $55,68 \mathrm{n} 8,105,125 \mathrm{n} 12$; formulas of rhythmic variation 53-54, 56; frequency $37,42,60-63,206$; narrative (or discourse) time 3,8 , 33-37, 48, 50, 53, 54, 55, 56, 60, $63,64,68 \mathrm{n} 9,69 \mathrm{n} 31,87$; 'panel is a moment' thesis 55; polychrony 63,70 n 42 ; retrospection 37,49 ; rhythm 22, 42, 45, 50-56, 57-58, $59,63,67,69$ n $32,79-80,232-37$, 265 ; story time $33-37,50,53,56$, $60,63,64,68 \mathrm{n} 9,69 \mathrm{n} 31,87$; stretch $53,54,56,143,236$; temporal order 33-50, 63; temporality in panel relations $38-45$; see also reading conventions

Tintin 11, 46, 90, 113, 115, 137, 186, 195, 208-09; L'Ile noire (The Black Island) 156-57; The Red Sea Sharks 42; Tintin au Tibet 208

Topolino (comic) 180, 194

transmedial storytelling, see intermediality and narratology, transmedial

Trondheim, Lewis 29n28, 70n41, 159

Töpffer, Rodolphe 99, 205-06, 222, 228, 230, 240-41n4, 248; "Essai de physiognomonie" 222

$V$ for Vendetta (by Alan Moore and David Lloyd) 73-74

Valentina (by Guido Crépax) 36-37

Van Lier, Henri 43-44

Verstraten, Peter 11, 89n15

Walking Dead, The (by Robert Kirkman, Charlie Adlard, and Cliff Rathburn) 95-97, 99

Walking with Samuel (by Tommi Musturi) 113-14, 122-23, 160

Ware, Chris 23, 33, 35, 49, 51, 65, 67, $67 \mathrm{n} 2,69 \mathrm{n} 25,150,176$

Watchmen (by Alan Moore, Dave Gibbons and John Higgins) 42, 193

Waugh, Colton 13, 107n1

Weapons of Mass Diplomacy (by Abel Lanzac and Christophe Blain) 223-25

Williams, Charles 251, 255

Wolk, Douglas 17, 70n34

Woodward, George 251, 275n19, $275 \mathrm{n} 22$

wordless comics 15, 38, 40-41, 61, $75,79-81,83,88 \mathrm{n} 1,113-14,122$, $124,125 \mathrm{n} 16,153,160,161-62$, 169,170

writing: style of $112,113,133,221$, 226-27, 240, 248 